NBSIR 78-1479

\title{
Thermodynamic Data for Waste Incineration
}

Eugene S. Domalski, William H. Evans, and Thomas L. Jobe, Jr.

Chemical Thermodynamics Data Center

Chemical Thermodynamics Division

Center for Thermodynamics and Molecular Science

and the Office of Standard Reference Data

National Bureau of Standards

Washington, D.C. 20234 ,

August 1978

Prepared for:

American Society for Mechanical Engineers

Research Committee on Industrial and Municipal Wastes

$Q C \quad$ ited Engineering Center

100 iw York, N.Y.

. 456

no. $78-1479$

1978

C.2 


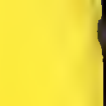


Eugene S. Domalski, William H. Evans, and Thomas L. Jobe, Jr.

Chemical Thermodynamics Data Center

Chemical Thermodynamics Division

Center for Thermodynamics and Molecular Science and the Office of Standard Reference Data

National Bureau of Standards

Washington, D.C. 20234

August 1978

Prepared for:

American Society for Mechanical Engineers

Research Committee on Industrial and Municipal Wastes United Engineering Center

New York, N.Y.

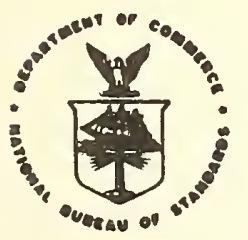

U.S. DEPARTMENT OF COMMERCE, Juanita M. Krops, Socrotary

Dr. Sidney Harman, Under Secrotery

Jordan J. Baruch. Assistant Secrotary for Scionce and Tochnology

NATIONAL BUREAU OF StANDARDS. Emeot Ambler, Diroctor 
Incinerators have continued to play an important part in reducing the amount and modifying the type of waste discharged to the environment.

The ASME Research Committee on Industrial and Municipal Wastes was established in 1968. This committee recognized the need for greater understanding of combustion fundamentals if engineers are to design better incinerators to meet the challenge of improving the environment. It, therefore, created a subcommittee on fundamental combustion studies.

The first report of the subcommittee, published in 1974, was entitled: Combustion Fundamentals for Waste Incineration. The purpose of the report was to help engineers make better calculations and thereby design better incinerators. The report was divided into two parts. Part one covered theoretical engineering methods of calculation and tables of equilibrium products of combustion with practical examples. Part two included the scientific theory and tables of thermodynamic properties.

This is the second report of the subcomittee. The purpose of this report is to provide engineers with thermodynamic data on materials which are mixtures of various kinds and are often difficult to describe using a single stoichiometric formula. These materials (i.e., animals, foods, plants, polymers, wood species, etc.) are encountered perhaps more often by engineers engaged in the disposal of municipal wastes than pure substances. The bulk of the data consist of gross heating values and specific heats, and should be helpful in combustion calculations.

The report is dedicated to all engineers involved in designing, procuring, operating, and managing incinerators. 

INTRODUCION

UNITS AND DEFINITIONS

vi

TABLE OF THERMODYNAMIC PROPERIIES

OF MISCELIANEOUS MATERTALS

1

MATERIAL NAME - PROPERTY INDEX

117

REFERENCES

141

APPENDIX

151 


\section{Introduction}

The general purpose of this table of thermodynamic data of selected materials is to make property information available to the engineering comunity on chemical mixtures, polymers, composite materials, solid wastes, and materials not easily identifiable by a single stoichiometric formula. Workers in many sectors need this type of information: construction engineers who require thermodynamic information on materials, safety engineers assessing the hazard potential of chemicals, cryogenic engineers involved in low temperature research, chemical engineers concerned with industrial unit processes, nitritionists interests in the heating values of foods, and scientists who want thermodynamic data for specific research projects.

The specific purpose of this table is to provide incinerator engineers with appropriate thermodynamic data so that they can dispose of waste materials in a more effective and environmentally acceptable manner. The effective disposal of waste materials is an important part of the maintenance of a high quality environment and incineration offers the most significant means of volume reduction for waste materials when compared to other methods of disposal.

This table of thermodynamic data was prepared in the Chemical Thermodynamics Data Center of the National Bureau of Standards and was administered through the NBS Office of Standard Reference Data over the period 1 January 1975 to 31 December 1976. A total of 331 materials have been compilel covering properties such as: specific heat, heat of combustion, heat of fusion, heat of vaporization, heat of explosion, vapor pressure, and explosion temperature. The information was obtained from the master files of the NBS Chemical Thermodynamics Data Center, the annual issues of the Bulletin of Thermodynamics and Thermochemistry, intermittent examinations of the Chemical Abstracts Subject Indexes, individual articles by various authors, and other general reference sources.

The basic format of the table is alphabetical according to the name of the materlal; similar materials, such as foods, polymers, woods, and explosives are grouped under the broader classification. For each materlal the following are given (1) the physical state, (2) information as to the composition of the material, (3) the kind of thermodynamic property reported, (4) the specific property values for the material, and (5) citations to the reference list. An index arranged by subject and property is provided which will assist a user in finding a specific mater la1 or property. 
Units and Definitions

The notation in the tables for thermodynamic quantities follows, in general, that in "Combustion Fundamentals for Waste Incineration" [141]. In those tables values were reported on a molar basis. Here, because of the lack of definite stolchiometric compositions for most of the materials included, values of the thermodynamic quantities are given on a specific basis, for one gram of substance. Exceptions are noted. The symbols used are briefly defined below; more extensive discussions are given in [141].

T. The absolute temperature, on the International Practical Temperature Scale. Values are given in kelvins (symbol K). The Celsius ("Centigrade") scale (symbol ${ }^{\circ} \mathrm{C}$ ) is used to indicate the temperature or temperature range for many reported measurements. It $1 \mathrm{~s}$ defined as $t\left({ }^{\circ} \mathrm{C}\right)=\mathrm{T}(\mathrm{K})-273.15$. The Fahrenheit scale, involved in a few measurements, is defined as $t\left({ }^{\circ} \mathrm{F}\right)=1.8 \mathrm{t}^{\circ} \mathrm{C}+32$. Unless specificaliy indicated otherwise, all temperatures $\mathrm{T}$ involved in equations are absolute temperature ( $T, K$ ).

$q_{v}$ (gross). The specific isothermal gross heat of reaction at the specified temperature and at constant volume. $q_{v}$ (gross) is equal to $-\Delta U / M$, where $\Delta U$ is the molar change in internal energy of the process and $M$ is the molecular weight. Where possible, the values of $\mathrm{qv}$ (or $\Delta \mathrm{U}$ ) have been converted to the standard state $\left(-\Delta U^{\circ} / M\right)$ [141]. Most of the data, though, are of such low precision, because of sample varlability or measurement method, that this correction is impractical. For combustion reactions in oxygen the products are taken as $\mathrm{H}_{2} \mathrm{O}(1), \mathrm{CO}_{2}(\mathrm{~g}), \mathrm{N}_{2}(\mathrm{~g}), \mathrm{HCl}$ (aq, dilute), $\mathrm{H}_{2} \mathrm{SO}_{4}$ (aq, dilute). Other products are identified. Metallic elements are assumed to form the oxide. For many of the substances considered, the metallic elements are included in the "ash" fraction; the values of qv(gross) may have been reported on the "ash-free" basis, which assumes a neglibile heat effect from the non-combustible material.

The numerical values are given in $\mathrm{J} / \mathrm{g}$ and cal/g. The calorie used is the thermochemical calorie, defined as $4.184 \mathrm{~J}$. Values originally reported in other units, such as $B T U / 1 b$, have been systematically converted to J/g. Conversion factors are given in the Physical Constant Table.

$q_{v}$ (net). The specific isothermal net heat of reaction at the specified temperature and at constant volume. Differs from $y_{v}$ (gross) in that
water formed is in the gaseous state. 
$q_{p}$ (net). The specific 1sothermal net heat of reaction at the specifled temperature and at constant pressure. $q_{p}$ (net) is equal to $-\Delta H / M$, where $\Delta H$ is the molar change in enthalpy of the process and $M$ is the molecular welght. Where possible, the values of $q_{p}(n e t)$ have been converted to the standard state, $\left(-\Delta H^{\circ} / \mathrm{M}\right)$ [141]. Most of the data, though, are of such low prec1sion, because of sample varlability or measurement method, that the correction is impractical. Unless indicated otherwise, the products of combustion in oxygen or of explosion are taken as $\mathrm{CO}_{2}(\mathrm{~g}), \mathrm{H}_{2} \mathrm{O}(\mathrm{g}), \mathrm{N}_{2}(\mathrm{~g})$; other products are Indicated. For explosion heats, the products are not reported in many cases; in some cases, reactions have been assumed, based on data for similar substances.

$\mathrm{L}_{\mathrm{f}}$. The specific latent heat of fusion $\left(\Delta \mathrm{H}_{\mathrm{fus}} / \mathrm{M}\right.$ ) at a pressure of I atm (101325 Pa). The value is the heat absorbed (in $\mathrm{J}$ and cal) when one gram of the substance goes from the solld state to the liquid state. For a pure compound, the melting occurs at a single temperature; for the complex materlals considered here, the melting process may take place over a temperature range.

$\mathrm{L}_{t}$. The specific latent heat of transition ( $\Delta \mathrm{H}_{\mathrm{trs}} / \mathrm{M}$ ) at a pressure of 1 atm (101325 Pa). The value is the heat absorbed when one gram of the substance goes from one solld phase to another solid phase. For a pure compound, the transition occurs at a single temperature; for the complex materials considered here, the transition process may take place over a temperature range.

$L_{v}$. The specific latent heat of vaporization $\left(\Delta \mathrm{H}_{\mathrm{vap}} / \mathrm{M}\right)$. The value is the heat absorbed when one gram of the substance goes from the 11quid state to the gaseous state. If measured calorimetrically the value w111 be for a definite temperature and pressure. If dertved from the vapor pressure equation through the relation $d(\ln P) / d(1 / T)=-\Delta H / R$, the value may be referred to a definite temperature and pressure, depending upon the form of the equation. With the simple form $\ln \mathrm{P}=\mathrm{A}-\mathrm{B} / \mathrm{T}$, the value refers to the mIdpoint of the temperature range over which the equation is valld. The value thus obtained is for one mole of gaseous specles; the average molecular weight must be obtained to calculate the specific heat of vaporization: $\mathrm{L}_{\mathrm{v}}=$ $\Delta \mathrm{H}_{\mathrm{v}} / \mathrm{M}$. 
$\mathrm{L}_{\mathrm{s}}$. The specific latent heat of sublimation $\left(\Delta \mathrm{H}_{\mathrm{sub}} / \mathrm{M}\right)$. The value is the heat absorbed when one gram of the substance goes from the solid state to the gaseous state. The same considerations apply as for $\mathrm{I}_{\mathrm{v}}$.

Heat of combustion. The change in the internal energy $\left(-q_{v}\right)$ or enthalpy $\left(-q_{p}\right)$ when one gram of the substance is burned 1sothermally in oxygen. Bsually measured by combustion in a calorimetric bomb at elevated oxygen pressure. Gross heat of combustion refers to a process in which the major products are $\mathrm{CO}_{2}(\mathrm{~g}), \mathrm{H}_{2} \mathrm{O}(1), \mathrm{N}_{2}(\mathrm{~g})$. Net heat of combustion refers to $\mathrm{H}_{2} \mathrm{O}(\mathrm{g})$ as a product.

In accurate work the observed heat is corrected for side reactions and to standard state in which all gases are at unit fugacity; for most of the data reported here, the precision of the measurements does not warrent such refinement.

Heat of explosion. The isothermal heat evolved during the explosive decomposition of the substance. All gaseous products, including water, are assumed to be at $1 \mathrm{~atm}$ pressure. The products are a complex mixture; in many cases, a quantitative description of the reaction is not possibly [see ref. 77].

Specific heat. The change in enthalpy with temperature at constant pressure (I atm unless otherwise speclfled) for one gram of substance. $c_{p}=(\partial(H / M) / \partial T) p$. At constant volume $c_{V}=(\partial(U / M) / \partial T)$.

Vapor pressure. The saturated equllibrium pressure over the liquid substance at a given temperature. Values are expressed in pascals $\left(\mathrm{Pa}=\mathrm{N} \cdot \mathrm{m}^{-2}\right.$ ) and 1n Torr (Torr $=133.3224 \mathrm{~Pa}$ ). The conventional atmosphere is taken as $101325 \mathrm{~Pa}$.

Sublimation Pressure. The saturated equilibrlum pressure over the solid substance at a glven temperature. Values are expressed in pascals $\left(\mathrm{Pa}=\mathrm{N} \cdot \mathrm{m}^{-2}\right.$ ) and in Torr (Torr $=133.3224 \mathrm{~Pa}$ ). The conventional atmosphere is taken as $101325 \mathrm{~Pa}$. 
Explosion temperature. A measure of the thermal stability of an explosive. The values 11sted in [77] were determined by the following procedure: "A 0.02-gm sample (0.01-g in the case of initiatiors) of explosive, loosely loaded in a No. 8 blasting cap, is immersed for a short period in a Wood's metal bath. The temperature determined is that which produces explosion, ignition or decomposition of the sample in 5 second." [77]

Heat content. The difference in enthalpy, for one gram of substance, between two temperatures, $\left(H_{T}-H_{T}(\right.$ ref $) / M=\left(q_{T}-q_{T}(\right.$ ref $)$. $T($ ref $)$ is the reference temperature, usually $0 \vec{K}, 273.15 \mathrm{~K}$, or $298.15 . \mathrm{K}$. It is specified as $q_{0}, q_{273}$ or $q_{273}, 15$, and $q_{298}$ or $q_{298,15}$. If other then the kelvin scale is used, this is indicated as ${ }^{\circ} \mathrm{C},{ }^{8} \mathrm{~F}$, or ${ }^{\circ} \mathrm{R}$ (Rankine, $\mathrm{T}^{\circ} \mathrm{R}=1.8 \mathrm{~T} \mathrm{~K}$ ).

Uncertainties. No attempt has been made to assess the over-all uncertainty of the data included. For some substances, uncertainties are given for the values; these represent the original authors' estimates, and are usually a measure of the reproducibility of their measurements. A large assigned uncertalnty usually indicates a variability of sample, not a low precision of measurement.

Multiple sources of data. Occasionally, several references are found in the literature which report the same thermodynamic property for a particular material. The measured values are most often not equivalent and a selection must be made as to which value is preferred. For some materials (such as coals), the values tabulated are typical values selected from a large number of results for samples of a similar nature. The selection process is based upon the personal and professional opinion of an evaluator after all of the property information for a material has been collected and examined. Factors which are taken into consideration are: (1) description of the method used to carry out the measurements, (2) characterization of the material, (3) number of measurements performed, (4) treatment of the experimental data, (5) dispersion of the results, and (6) background or experience of the laboratory personnel carrying out the measurements. 



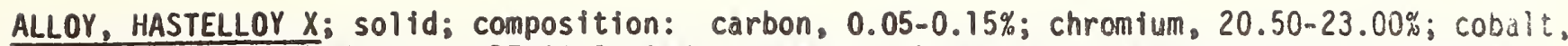
0.05-2.50\%; iron, 17.00-20.00\%; manganese, 1.00\% max.; m0 3ybdenum, 8.00-10.00\%; silicon, 1.00\% max.; tungsten, 0.2-1.0\%; remainder nickel, 42.0-54.0\%.

specific heat:

$$
\begin{aligned}
0-1100{ }^{\circ} \mathrm{C} & \\
\mathrm{Cp}\left(\operatorname{cal} \mathrm{g}^{-1} \mathrm{~K}^{-1}\right)= & 0.0924+4.438 \times 10^{-5} \mathrm{~T} \\
& -2.812 \times 10^{-9} \mathrm{~T}^{2}+1.030 \times 10^{-11} \mathrm{~T}^{3} \\
& -1.885 \times 10^{-14} \mathrm{~T}^{4}+1.38 \times 10^{-17} \mathrm{~T}^{5} \\
\mathrm{Cp}\left(\mathrm{J} \mathrm{g}{ }^{-1} \mathrm{~K}^{-1}\right)= & 0.3866+1.8569 \times 10^{-4} \mathrm{~T} \\
& -1.1765 \times 10^{-8} \mathrm{~T}^{2}+4.310 \times 10^{-11} \mathrm{~T}^{3} \\
& -7.887 \times 10^{-14} \mathrm{~T}^{4}+5.77 \times 10^{-17} \mathrm{~T}^{5}
\end{aligned}
$$

heat content:

$$
\begin{aligned}
& 0-1100{ }^{\circ} \mathrm{C} \\
& \begin{aligned}
q_{T}-q_{273.2}\left(\mathrm{cal} \mathrm{g} \mathrm{g}^{-1}\right)= & 0.0924 T+2.219 \times 10^{-5} \mathrm{~T}^{2} \\
& -9.373 \times 10^{-9} \mathrm{~T}^{3}+2.575 \times 10^{-12} \mathrm{~T}^{4} \\
& -3.770 \times 10^{-15} \mathrm{~T}^{5}+2.30 \times 10^{-18} \mathrm{~T}^{6}-27.1429
\end{aligned} \\
& \begin{aligned}
q_{T}-q_{273.2}\left(\mathrm{~J} \mathrm{~g}^{-1}\right)= & 0.3866 \mathrm{~T}+9.284 \times 10^{-5} \mathrm{~T}^{2} \\
& -3.922 \times 10^{-8} \mathrm{~T}^{3}+1.0774 \times 10^{-11} \mathrm{~T}^{4}
\end{aligned} \\
& -1.5774 \times 10^{-14} T^{5}+9.62 \times 10^{-18} T^{6}-113.5659
\end{aligned}
$$

ALLOY, 80 NICKEL-20 CHROMIUM; solid; composition: nickel, 77.4\%; chromium, 19.52\%; iron, $0.45 \%$; maganese, $0.59 \%$; silicon, $1.4 \%$; carbon, $0.04 \%$.

specific heat:

$0-150^{\circ} \mathrm{C}$

$\begin{array}{ll}\operatorname{Cp}\left(\operatorname{cal} g^{-1} K^{-1}\right) & =0.0825+7.6 \times 10^{-5} \mathrm{~T} \\ \operatorname{Cp}\left(J g^{-1} K^{-1}\right) & =0.3452+3.18 \times 10^{-4} \mathrm{~T}\end{array}$

$\mathrm{Cp}\left(\mathrm{J} \mathrm{g}^{-1} \mathrm{~K}^{-1}\right)$

$150-600{ }^{\circ} \mathrm{C}$

$\begin{array}{ll}\operatorname{Cp}\left(\mathrm{cal}_{1} \mathrm{~g}^{-1} K^{-1}\right) & =0.0952+4.64 \times 10^{-5} \mathrm{~T} \\ \operatorname{Cp}\left(J \mathrm{~g}^{-1} K^{-1}\right) & =0.3983+1.941 \times 10^{-4} \mathrm{~T}\end{array}$

$600-900{ }^{\circ} \mathrm{C}$

$C p\left(\operatorname{cal} g^{-1} K^{-1}\right)=0.1021+4.62 \times 10^{-5} \top$
$C p\left(J g K^{-1}\right)=0.4271+1.933 \times 10^{-4} T$ 
ALLOY, TITANIUM, Ti-75A; solid; composition: titanium, 99.75\%; iron, 0.07\%; oxygen, $0.131 \%$; nitrogen, $0.048 \%$; carbon, $0.06 \%$; hydrogen, $0.0068 \%$.

specific heat:

$21-760{ }^{\circ} \mathrm{C}$

$\begin{array}{ll}\left.C p(c a) g^{-1} K^{-1}\right) & =0.1384-9.1526 \times 10^{-5} \mathrm{~T}+1.555 \times 10^{-7} \mathrm{~T}^{2} \\ C P\left(J g^{-1} K^{-1}\right) & =0.5791-3.8294 \times 10^{-4} \mathrm{~T}+6.506 \times 10^{-7} \mathrm{~T}^{2}\end{array}$

ALLOY, TITANIUM, Ti-150A; solid; composition: titanium, 95.65\%; chromium, $2.71 \%$; iron, 1.40\%; oxygen, $0.105 \%$; nitrogen, $0.076 \%$; carbon, $0.05 \%$; hydrogen, $0.0092 \%$.

specific heat:

$21-760{ }^{\circ} \mathrm{C}$

$\operatorname{Cp}\left(\operatorname{cal} g^{-1} K^{-1}\right)=0.1434-9.1526 \times 10^{-5} \mathrm{~T}+1.555 \times 10^{-7} \mathrm{~T}^{2}$
$\operatorname{Cp}\left(\mathrm{J} \mathrm{g} \mathrm{K}^{-1}\right)=0.6000-3.8294 \times 10^{-4} \mathrm{~T}+6.506 \times 10^{-7} \mathrm{~T}^{2}$

ALLOY, ZIRCALOY-2; solid, composition: tin, 1.46\%; iron, $0.130 \%$; chromium, $0.080 \%$; nicke1, 0.056\%; carbon, 65 ppm; oxygen, 1489 ppm; nitrogen, 40 ppm; hydrogen, $11 \mathrm{ppm}$; copper, $20 \mathrm{ppm}$; tungsten, <20 ppm; aluminum, $40 \mathrm{ppm}$; silicon, $47 \mathrm{ppm}$; hafnium; <125. ppm; remainder is zirconium.

specific heat:

$0-635^{\circ} \mathrm{C}$

$\begin{array}{ll}\operatorname{Cp}\left(\operatorname{cal} g^{-1} K^{-1}\right) & =0.06153+2.3872 \times 10^{-5} \mathrm{~T} \\ \operatorname{Cp}\left(J g^{-1} K^{-1}\right) & =0.25744+9.9880 \times 10^{-5} \mathrm{~T}\end{array}$

$635-810^{\circ} \mathrm{C}$

$\begin{array}{ll}C p\left(\operatorname{cal} g^{-1} K^{-1}\right) & =0.08589 \\ C p\left(J g^{-1} K^{-1}\right) & =0.35936\end{array}$

$975-1050{ }^{\circ} \mathrm{C}$

$\operatorname{Cp}\left(\operatorname{cal} g^{-1} K^{-1}\right)=0.08548$
$\operatorname{Cp}\left(\mathrm{J} \mathrm{g}^{-{ }^{-1}} K^{-1}\right)=0.35765$

heat of transition: temperature range: $810-975{ }^{\circ} \mathrm{C}$, for the $\alpha-\beta$ transition $L_{t}=10.6 \mathrm{cal}^{-1}$
$L_{t}=44.4 \mathrm{Jg}^{-1}$ 
ALLOY, ZIRCALOY-2 (LOW NICKEL); solid; composition: tin, 1.32\%; iron, 0.152\%; chromium, $0.99 \%$; nickel, $10 \mathrm{ppm}$; carbon, $150 \mathrm{ppm}$; oxygen, 1380 ppm; nitrogen, $36 \mathrm{ppm}$; copper, $20 \mathrm{ppm}$; tungsten, $<40 \mathrm{ppm}$; hafnium, $60 \mathrm{ppm}$; remainder is zirconium.

specific heat:

$0-570{ }^{\circ} \mathrm{C}$

$\begin{array}{ll}C p\left(\operatorname{cal} g^{-1} K^{-1}\right) & =0.06314+2.0726 \times 10^{-5} \mathrm{~T} \\ C p\left(\mathrm{Jg}^{-1} \mathrm{~K}^{-1}\right) & =0.26418+8.6718 \times 10^{-5} \mathrm{~T}\end{array}$

$600-810^{\circ} \mathrm{C}$

$C p\left(C a l g^{-1} K^{-1}\right)=0.08571$

$\mathrm{CP}\left(\mathrm{J} \mathrm{g}^{-1} \mathrm{~K}^{-1}\right)=0.35861$

$975-1050{ }^{\circ} \mathrm{C}$

$C p\left(C a l-g^{-1} K^{-1}\right)=0.08548$

$\mathrm{Cp}\left(\mathrm{J} \mathrm{g}^{-1} \mathrm{~K}^{-1}\right)=0.35765$

[80]

ANIMALS

BARNACLE, Balanus cariosus; solid; invertebrate animal; crustacean of the order Cirripedia.

gross heat of combustion: assume values refer to room temperature and are corrected for moisture and ash content.

$q_{v}($ gross $)=4520 \mathrm{cal} \mathrm{g}^{+1}$
$q_{v}($ gross $)=18912 \mathrm{~J} \mathrm{~g}^{1}$

BEETLE, Tenebrio molitor; solid; an insect of the order Coleoptera, having four wings the outer pair being stiff cases which cover the others when they are folded.

gross heat of combustion: assume values refer to room temperature and are corrected for molsture and ash content. .

$q_{v}($ gross $)=6314 \pm 516 \mathrm{cal} \mathrm{g}^{-1}$
$q_{v}($ gross $)=26418^{-1} \pm 2159 \mathrm{~J} \mathrm{~g}^{-1}$

[75]

BIVALVE, Mytilus californianus; solid; invertebrate animal composed of two parts which open and shut, such as oysters and clams; mollusks.

gross heat of combustion: assume values refer to room temperature and are corrected for moisture and ash content.

$q_{v}($ gross $)=4600 \mathrm{cal} \mathrm{g}^{-1}$
$q_{v}($ gross $)=19246 \mathrm{~J} \mathrm{~g}^{1}$ 
BLOOD, SHEEP; solid; Protein and fat composition determined on ash-free dry-basis for materials from bodies of 63 sheep; mean composition, protein, $98.80 \%$; fat, $1.20 \%$.

gross heat of combustion: assume values refer to room temperature.

$q_{v}($ gross $)=5873 \mathrm{cal} \mathrm{g}^{-1}$
$q_{v}($ gross $)=24573 \mathrm{~J} \mathrm{~g}^{2}$

For the \% fat range 0.00 to 5.80 :

$q_{v}($ gross $)=(5854+15.83(\%$ fat $)) \mathrm{cal} \mathrm{g}^{-1}$
$q_{v}($ gross $)=(24493+66.23(\%$ fat $)) \mathrm{J} \mathrm{g}^{1}$

For the $\%$ protein range 94.20 to 100.00 :

$q_{v}($ gross $)=(7435-15.83(\%$ protein $)) \mathrm{cal} \mathrm{g}^{-1}$
$q_{v}($ gross $)=(31108-66.23(\%$ protein $)) \mathrm{Jg}^{-1}$

BRACHIOPOD, Glottidia pyramidata; solid; a class of Molluscoida having bivalve shells.

gross heat of combustion: assume values refer to room temperature and are corrected for moisture and ash content.

$q_{v}($ gross $)=4397 \pm 2140 \mathrm{cal} \mathrm{g}^{-1}$
$q_{v}($ gross $)=18397 \pm 8954 \mathrm{~J} \mathrm{~g}^{1}$

CARCASS, SHEEP; solid; Protein and fat composition determined on ash-free dry basis for carcasses of 63 sheep; mean composition, protein, $40.18 \%$, fat, $59.82 \%$.

gross heat of combustion: assume values refer to room temperature.

$q_{v}($ gross $)=7778 \mathrm{cal} \mathrm{g}^{-1}$
$q_{v}($ gross $)=32543 \mathrm{~J} \mathrm{~g}^{1}$

For the \% fat range 33.32 to 81.69 :

$q_{v}($ gross $)=(5327+40.97(\%$ fat $)) \mathrm{cal}^{-1}$
$q_{v}($ gross $)=(22288+171.42(\%$ fat $)) \mathrm{J} \mathrm{g}^{-1}$

For the \% protein range 18.31 to 66.68 :

$q_{v}($ gross $)=(9424-40.97(\%$ protein $)) \mathrm{cal}^{g^{-1}}$
$q_{v}($ gross $)=(39430-171.42(\%$ protein $)) \mathrm{J} \mathrm{g}^{-1}$ 
CATTLE; solid; results apply to total body of cattle.

gross heat of combustion: assume values refer to room temperature and are corrected for moisture and ash content.

For sample of $100 \%$ protein:

$q_{v}($ gross $)=5447 \mathrm{cal} \mathrm{g}^{-1}$
$q_{v}($ gross $)=22790 \mathrm{~J} \mathrm{~g}^{1}$

For sample of $100 \%$ fat:

$q_{v}($ gross $)=9499 \mathrm{cal} \mathrm{g}^{-1}$
$q_{v}($ gross $)=39744 \mathrm{~J} \mathrm{~g}^{1}$

CHITON, Katherina tunicata; solid; invertebrate animal; mollusk of the order Polyplacophora.

gross heat of combustion: assume values refer to room temperature and are corrected for moisture and ash content.

$q_{v}($ gross $)=4700 \mathrm{cal} \mathrm{g}^{-1}$
$q_{v}($ gross $)=19665 \mathrm{~J} \mathrm{~g}^{1}$

CILIATE, Tetrahymena pyriformis; solid; class of Protozoa characterized by presence of cilla and found in any exposed body of water.

gross heat of combustion: assume values refer to room temperature and are corrected for moisture and ash content.

$q_{v}($ gross $)=5938 \pm 207 \mathrm{cal} \mathrm{g}^{-1}$
$q_{v}($ gross $)=24845 \pm 866 \mathrm{~J} \mathrm{~g}^{1}$

CLADOCERA, Leptodora kindtu; solid; a group of minute, chiefly fresh-water, entomostracan crustaceans, often known as water fleas.

gross heat of combustion: assume values refer to room temperature and are corrected for moisture and ash content.

$q_{v}($ gross $)=5605 \pm 584 \mathrm{cal} \mathrm{g}^{-1}$
$q_{v}($ gross $)=23451 \pm 2443 \mathrm{~J} \mathrm{~g}^{-1}$ 


\section{ANIMALS}

COPEPOD, Calanus helgolandicus; solid; a large subclass of crustaceans mostly minute in size found in both fresh and salt water.

gross heat of combustion: assume values refer to room temperature and are corrected for moisture and ash content.

$q_{v}($ gross $)=5400 \pm 197 \mathrm{cal} \mathrm{g}^{-1}$
$q_{v}($ gross $)=22594 \pm 824 \mathrm{~J} \mathrm{~g}^{2}$

COPEPOD, Trigriopus californicus; solid; a large subclass of crustaceans mostly minute in size and found in both fresh and salt water.

gross heat of combustion: assume values refer to room temperature and are corrected for moisture and ash content.

$q_{v}($ gross $)=5515 \pm 277 \mathrm{cal} \mathrm{g}^{-1}$
$q_{v}($ gross $)=23075 \pm 1159 \mathrm{~J} \mathrm{~g}^{-1}$

FLATWORM, Dugesia tigrina; solid; a planarian worm of the phylum Platyhelminthes.

gross heat of combustion: assume values refer to room temperature and are corrected for moisture and ash content.

$q_{v}($ gross $)=6286 \pm 338 \mathrm{cal}^{-1}$
$q_{v}($ gross $)=26301 \pm 1414 \mathrm{~J} \mathrm{~g}^{-1}$

FLATWORM, TERRESTRIAL, Bipalium keuense; solid; large worm found in the tropics, having the head end expanded into a semicircular plate.

gross heat of combustion: assume values refer to room temperature and are corrected for moisture and ash content.

$q_{v}($ gross $)=5684 \pm 124 \mathrm{cal} \mathrm{g} \mathrm{g}^{-1}$
$q_{v}($ gross $)=23782 \pm 519 \mathrm{~J} \mathrm{~g}^{1}$

FLY, CADDIS, Pycnopsyche guttifer; solid; insects having aquatic larvae, they are included in the Neuroptera, or constitute the order Trichoptera; they have four membranous wings densely hairy, vestigial mouth parts and many jointed antennas.

gross heat of combustion: assume values refer to room temperature and are corrected for moisture and ash content.

$q_{v}($ gross $)=5706 \mathrm{cal} \mathrm{g} \mathrm{g}^{-1}$
$q_{v}($ gross $)=23874 \mathrm{Jg}^{1}$ 


\section{ANIMALS}

FLY, CADDIS, Pycnopsyche lepido; solid; insects having aquatic larvae, they are included in the Neuroptera, or constitute the order Trichoptera, they are densely hairy, have four membranous wings, vestigial mouth parts and many jointed antennae.

gross heat of combustion: assume values refer to room temperature and are corrected for molsture and ash content.

$q_{v}($ gross $)=5687 \pm 530 \mathrm{cal} \mathrm{g}$
$q_{v}($ gross $)=23794 \pm 2218 \mathrm{Jg}^{-1}$

GUPPIE, Lebistes reticulatus; solid; a small top minnow of the Barbados, Trinidad and Venezeula area; frequently kept as an aquarium fish.

gross heat of combustion: assume values refer to room temperature and are corrected for moisture and ash content.

$q_{v}($ gross $)=5823 \mathrm{cal} \mathrm{g}_{1}^{-1}$
$q_{v}($ gross $)=24363 \mathrm{~J} \mathrm{~g}^{-1}$

HIDE, SHEEP; solid; Protein and fat composition determined on ash-free dry basis for material from bodies of 63 sheep; mean composition, protein, 74.05\%, fat, 25.95\%.

gross heat of combustion: assume values refer to room temperature.

$q_{v}($ gross $)=6473 \mathrm{cal} \mathrm{g}^{-1}$
$q_{v}($ gross $)=27083 \mathrm{~J} \mathrm{~g}^{-1}$

For the $\%$ fat range 18.37 to 38.72 :

$q_{v}($ gross $)=(5458+38.47(\%$ fat $)) \mathrm{cal}^{-1} \mathrm{~g}^{-1}$
$q_{v}$ (gross $)=(22836+160.96(\%$ fat $)) \mathrm{Jg}^{-1}$

For the \% protein range 61.28 to 81.63 :

$q_{v}($ gross $)=(9305-38.47(\%$ protein $)) c a l^{-1}$
$q_{v}($ gross $)=(38932-160.96(\%$ protein $)) \mathrm{J} \mathrm{g}^{-1}$

HYDRA, Hydra littoralis; solid; small fresh-water hydrozoan polyps usually found attached to submerged objects, the body is a simple tabe with a mouth at one extremity.

gross heat of combustion: assume values refer to room temperature and are corrected for moisture and ash content.

$q_{v}($ gross $)=6034 \pm 146 \mathrm{cal} \mathrm{g}^{-1}$
$q_{v}($ gross $)=25246 \pm 61.1 \mathrm{~J} \mathrm{~g}^{-1}$ 


\section{ANIMALS}

HYDRA, GREEN, Chlorohydra viridissima; solid; small fresh-water hydrozoan polyps which contains chlorophyll chromatophores, usually found attached to submerged objects, the body is a simple tube with a mouth at one extremity.

gross heat of combustion: assume values refer to room temperature and are corrected for moisture and ash content.

$q_{v}($ gross $)=5729 \pm 247 \mathrm{cal} \mathrm{g}^{-1}$
$q_{v}($ gross $)=23970 \pm 1033 \mathrm{Jg}^{-1}$

MITE, Tyroglyphus lintneri; solid; small arachnids which are parasitic in nature.

gross heat of combustion: assume values refer to room temperature and are corrected for moisture and ash content.

$q_{v}($ gross $)=5808 \pm 446 \mathrm{cal} \mathrm{g}^{-1}$
$q_{v}^{y}($ gross $)=24301 \pm 1866 \mathrm{~J} \mathrm{~g}^{-1}$

SHEEP, INGESTA-FREE BODY; solid; protein and fat composition determined on ash-free dry basis for bodies of 63 sheep; mean composition, protein, $43.33 \%$, fat, $56.67 \%$.

gross heat of combustion: assume values refer to room temperature.

$q_{v}$ (gross) $=7661 \mathrm{cal} \mathrm{g}_{1}^{-1}$

$q_{v}($ gross $)=32054 \mathrm{~J} \mathrm{~g}^{2}$

For the \% fat range 28.80 to 79.43 :

$q_{v}($ gross $)=(5379+40.25(\%$ fat $)) \mathrm{cal} \mathrm{g}^{-1}$
$q_{v}($ gross $)=(22506+168.41(\%$ fat $)) \mathrm{J} \mathrm{g}^{-1}$

For the \% protein range 20.57 to 71.20 :

$q_{v}($ gross $)=(9405-40.25(\%$ protein $)) \mathrm{cal} \mathrm{g}^{-1}$
$q_{v}($ gross $)=(39351-168.41(\%$ protein $)) \mathrm{J} \mathrm{g}^{-1}$

SHRIMP, BRINE, Artenia SP. (nauplï); solid; a brachiopod crustacean.

gross heat of combustion: assume values refer to room temperature and are currected for moisture and ash content.

$q_{v}($ gross $)=6737 \pm 863 \mathrm{cal} \mathrm{g}^{-1}$
$q_{v}($ gross $)=28188 \pm 3611 \mathrm{~J} \mathrm{~g}^{-1}$ 


\section{ANIMALS}

SNAIL. Tegula funebralis; solid; invertebrate animal; gastropod mollusk.

gross heat of combustion: assume values refer to room temperature and are corrected for moisture and ash content.

$q_{v}($ gross $)=5080 \mathrm{cal} \mathrm{g}^{-1}$
$q_{v}($ gross $)=21255 \mathrm{~J} \mathrm{~g}^{1}$

SNAIL, AQUATIC, Succinea ovalis; solid; (without shell); a gastropod mollusk.

gross heat of combustion: assume values refer to room temperature and are corrected to an ash free basis.

$q_{v}($ gross $)=5415 \pm 6 \mathrm{cal} \mathrm{g}_{-1}^{-1}$
$q_{v}($ gross $)=22656 \pm 25 \mathrm{~g}^{-1}$

SPITBUG, Philenus leucophthalmus; solid; a spittle insect.

gross heat of combustion: assume values refer to room temperature and are corrected for molsture and ash content.

$q_{v}($ gross $)=6962 \pm 510 \mathrm{cal} \mathrm{g}^{-1}$
$q_{v}($ gross $)=29129^{-1} \pm 2134 \mathrm{~J} \mathrm{~g}^{-1}$

STARFISH; solid; Pisaster ochraceus; invertebrate animal; echinoderm of the order Asteroidea.

gross heat of combustion: assume values refer to room temperature and are corrrected for moisture and ash content.

$q_{v}($ gross $)=2110 \mathrm{cal}^{-g^{-1}}$
$q_{v}($ gross $)=8828 \mathrm{~J} \mathrm{~g}^{-1}$ 
ANIMALS

VISCERA, SHEEP; solid; protein and fat composition determined on ash-free dry-basis for viscera from bodies of 63 sheep; mean composition, protein, $33.50 \%$, fat, $66.50 \%$.

gross heat of combustion: assume values refer to room temperature.

$q_{v}$ (gross) $=8011 \mathrm{cal} \mathrm{g}^{-1}$
$q_{v}($ gross $)=33518 \mathrm{~J} \mathrm{~g}^{-1}$

For the \% fat range 26.62 to 87.12 :

$q_{v}($ gross $)=(5428+38.81(\%$ fat $))$ cal $^{-1}$
$q_{v}($ gross $)=(22711+162.38(\%$ fat $)) \mathrm{J} \mathrm{g}^{-1}$

For the? \% protein range $12.88-73.38$ :

$q_{v}$ (gross) $=(3312-38.81(\%$ protein $)) \mathrm{cal} \mathrm{g}_{-1}^{-1}$

$q_{v}^{v}($ gross $)=(38961-162.38(\%$ protein $)) \mathrm{J} \mathrm{g}^{-1}$

ASPHALT; solid; mixture of paraffinic, aromatic, and heterocyclic hydrocarbons (containing oxygen, nitrogen, and sulfur).

specific heat: Venezeulan distilled asphalt.

\begin{tabular}{|c|c|c|c|c|}
\hline \multirow{2}{*}{$\begin{array}{l}\text { Softening } \\
\text { Point, }{ }^{\circ} \mathrm{C}\end{array}$} & \multicolumn{4}{|c|}{$\left.C p(c a) g^{-1} k^{-1}\right)$} \\
\hline & $0^{\circ} \mathrm{C}$ & $100^{\circ} \mathrm{C}$ & $200^{\circ} \mathrm{C}$ & $300^{\circ} \mathrm{C}$ \\
\hline 39.5 & 0.425 & 0.472 & 0.520 & 0.567 \\
\hline $\begin{array}{l}62.5 \\
96.5\end{array}$ & $\begin{array}{l}0.409 \\
0.382\end{array}$ & $\begin{array}{l}0.463 \\
0.455\end{array}$ & $\begin{array}{l}0.518 \\
0.527\end{array}$ & $\begin{array}{l}0.5 / 2 \\
0.600\end{array}$ \\
\hline Softening & & & $\left.k^{-1}\right)$ & \\
\hline Point, ${ }^{\circ} \mathrm{C}$ & $0^{\circ} \mathrm{C}$ & $100^{\circ} \mathrm{C}$ & $200^{\circ} \mathrm{C}$ & $300^{\circ} \mathrm{C}$ \\
\hline 39.5 & 1.778 & 1.975 & 2.176 & 2.372 \\
\hline 62.5 & 1.711 & 1.937 & 2.167 & 2.393 \\
\hline 96.5 & 1.598 & 1.904 & 2.205 & 2.510 \\
\hline
\end{tabular}

$[94,95]$

BIPIIENYI. POLYCHLORINATED, CLOPHEN A30; liquid; $30 \%$ chlorine; product of Bayer-Leverkusen Company; average molecular weight $190 \pm 25$.

heat of vaporization: $40^{\circ}-160^{\circ} \mathrm{C}$

$I_{v}=96.45 \mathrm{cal}_{v}=403.5 \mathrm{Jg}^{-1}$

vapor pressure: $40^{\circ}-160^{\circ} \mathrm{C}$

$\log _{10} P(T u r r)=10.05-4005 \mathrm{~T}^{-1}$
$\log _{10} P(\mathrm{~Pa})=12.17-4005 \mathrm{~T}^{\mathrm{w}} \mathrm{I}$ 
BIPHENYL, POLYCHLORINATED, CLOPHEN A40; 1iquid; $40 \%$ chlorine; product of BayerLeverkusen Company; average molecular wefght $205 \pm 20$.

heat of vaporization: $40^{\circ}-160^{\circ} \mathrm{C}$

$L_{v}=92.29 \mathrm{cal}^{-g^{-1}}$
$L_{v}=386.1 \mathrm{Jg}^{-1}$

vapor pressure: $40^{\circ}-160^{\circ} \mathrm{C}$

$\log _{10} P($ Tor $r)=10.15-4135 \mathrm{~T}^{-1}$

$\log _{10} P(P a)=12.27-4135 \mathrm{~T}^{-1}$

BIPHENYL, POLYCHLORINATED, CLOPHEN A50; 11 quid; 50\% chlorine; product of Bayer-Leverkusen Company; average molecular weight $225 \pm 30$.

heat of vaporization: $40^{\circ}-160^{\circ} \mathrm{C}$

$L_{v}=88.46 \mathrm{car}_{v}-g^{-1}$
$L_{v}=370.1 \mathrm{Jg}^{-1}$

vapor pressure: $40^{\circ}-160^{\circ} \mathrm{C}$

$\log _{10} P(T o r r)=10.15-4350 \mathrm{~T}^{-1}$
$\log _{10} \mathrm{P}(\mathrm{Pa})=12.27-4350 \mathrm{~T}^{-1}$ 
(4-BRASS; solid; chemical composition in wt.\%; (sample 1): copper, $79.75 \%$; lead, $0.003 \%$; iron, $0.015 \%$; nickel, 0.01\%; zinc, 20.22\%; (sample 2): copper, $70.42 \%$; tin, $0.045 \%$; lead, $0.05 \%$; iron, $0.02 \%$; nicke1, $0.02 \%$; zinc, $29.445 \%$; (sample 3 ): copper, $65.18 \%$; lead, $0.001 \%$; iron, $0.002 \%$; nickel, $0.002 \%$; zinc, $34.815 \%$.

specific heat:

Temperature, $k$

$$
\operatorname{cp}\left(\operatorname{cal} \mathrm{g}^{-1} \mathrm{~K}^{-1}\right)
$$

$\operatorname{Cp}\left(\mathrm{J} \mathrm{g}^{-1} \mathrm{~K}^{-1}\right)$

sample 1

$\begin{array}{lll}298.15 & 0.0908 & 0.3798 \\ 400 & 0.0940 & 0.3935 \\ 500 & 0.1014 & 0.4243 \\ 600 & 0.1005 & 0.4205 \\ 700 & 0.1039 & 0.4347 \\ 800 & 0.1056 & 0.4418\end{array}$

sample 2

298.15

400

500

600

700

800

sample 3

298.15

400

500

600

700

800
0.0905

0.0943

0.1114

0.1011

0.1045

0.1064
0.3787

0.3946

0.4661

0.4230

0.4372

0.4452 
CELLULOID; solid; (trade mark, O Celanese Corp. of America); a solid solution of cellulose nitrate and camphor or other related plasticizers.

gross heat of combustion: assume values refer to room temperature.

$$
\begin{aligned}
& q_{v}(\text { gross })=4224 \mathrm{cal} \mathrm{g} \mathrm{g}_{1}^{-1} \\
& q_{v}(\text { gross })=17673 \mathrm{Jg}^{2}
\end{aligned}
$$

CEMENT HYDRATES; solid; general formula; $x \mathrm{CaO} \cdot y \mathrm{Al}_{2} \mathrm{O}_{3} \cdot 2 \mathrm{CaSO}_{4} \cdot m \mathrm{CaCO}_{3} \cdot n \mathrm{H}_{2} \mathrm{O} \cdot\left(\mathrm{C}=\mathrm{CaO}, A=A I_{2} \mathrm{O}_{3}\right.$, ${\mathrm{H}=\mathrm{H}_{2} \mathrm{O}}_{2} \mathrm{Cs}=\mathrm{CaSO}_{4}$, and $\left.\mathrm{CC}=\mathrm{CaCO}_{3}\right)$.

heat of dehydration: gaseous water formed.

\begin{tabular}{|c|c|c|c|c|}
\hline & temp range, ${ }^{\circ} \mathrm{C}$ & $\begin{array}{l}\text { moles } \mathrm{H}_{2} \mathrm{O} \\
\text { lost }\end{array}$ & (kca) $\left.\stackrel{\Delta H}{g n o l^{-2}}\right)^{-}$ & $\left(k J g_{\mathrm{mol}}^{\Delta H}{ }^{-1}\right)$ \\
\hline$C_{3.97^{A H} 11.0}$ & $\begin{array}{l}120-200 \\
210-290\end{array}$ & $\begin{array}{l}4 \\
3.5\end{array}$ & $\begin{array}{l}48.9 \pm 0.2 \\
10.2 \pm 0.2\end{array}$ & $\begin{array}{r}204.5 \pm 0.8 \\
42.7 \pm 0.8\end{array}$ \\
\hline $\mathrm{C}_{2.97^{\mathrm{AH}_{5.96}}}$ & $260-350$ & 4.5 & $76.9 \pm 1.8$ & $321.7 \pm 7.5$ \\
\hline 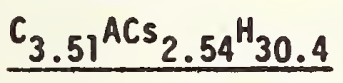 & $65-145$ & 23 & $213.9 \pm 3.1$ & $895.0 \pm 13.0$ \\
\hline$\frac{C_{3.14^{A C s}} 0.94^{\mathrm{H}} 12.1}{.}$ & $\begin{array}{r}95-125 \\
140-200 \\
240-310\end{array}$ & $\begin{array}{l}1.6 \\
6 \\
1.5\end{array}$ & $\begin{array}{l}2.75 \pm 0.2 \\
34.4 \pm 0.7 \\
14.6 \pm 0.6\end{array}$ & $\begin{array}{r}11.51 \pm 0.8 \\
143.9 \pm 2.9 \\
61.1 \pm 2.5\end{array}$ \\
\hline $\mathrm{C}_{3.53^{\mathrm{ACCH}_{8.1}}}$ & $\begin{array}{r}70-170 \\
250-320\end{array}$ & $\begin{array}{l}2 \\
5\end{array}$ & $\begin{array}{l}19.7 \pm 2 \\
31.0 \pm 0.5\end{array}$ & $\begin{array}{c}82.4 \pm 8 \\
129.7 \pm 2.1\end{array}$ \\
\hline$C_{1.97^{\mathrm{AH}} 12.9}$ & $\begin{array}{l}120-160 \\
230-325\end{array}$ & $\begin{array}{l}5.9 \\
4.8\end{array}$ & $\begin{array}{r}2.4 \pm 0.7 \\
76.0 \pm 5.8\end{array}$ & $\begin{array}{c}10.0 \pm 2.9 \\
318.0 \pm 24.1\end{array}$ \\
\hline $\mathrm{C}_{2.19^{\mathrm{AH}} 9.3}$ & $\begin{array}{r}45-100 \\
125-190 \\
250-300\end{array}$ & $\begin{array}{l}3 \\
1.5 \\
3.5\end{array}$ & $\begin{array}{r}12.9 \pm 2.7 \\
14.6 \pm 2.3 \\
6.0 \pm 0.7\end{array}$ & $\begin{array}{l}54.0 \pm 11.3 \\
61.1 \pm 9.6 \\
25.1 \pm 2.9\end{array}$ \\
\hline$c_{1.07^{A H_{6.3}}}$ & $\begin{array}{l}120-190 \\
230-300\end{array}$ & $\begin{array}{l}2 \\
3\end{array}$ & $\begin{array}{l}11.2 \pm 1.9 \\
22.3 \pm 1.0\end{array}$ & $\begin{array}{l}46.9 \pm 7.9 \\
93.3 \pm 4.2\end{array}$ \\
\hline$C_{1.03^{A H_{3}}}$ & $240-340$ & 2.8 & $43.7 \pm 2.7$ & $182.8 \pm 11.3$ \\
\hline
\end{tabular}


CHARCOAL, WOUD; solid; Ultimate analysis: carbon, $93.0 \%$; hydrogen, $2.5 \%$; nitrogen and sulfur, $1.5 \%$; moisture, $2 \%$; proximate analysis: fixed carbon, $89 \%$; ash, $1 \%$; volatile matter, $10 \%$.

gross heat of coinbustion: assume values refer to room temperature.

ash free and moisture free

$q_{v}($ gross $)=8300 \mathrm{cal} \mathrm{g}^{-1}$
$q_{v}($ gross $)=34727 \mathrm{Jg}^{1}$

as received; "good commercial fuel"

$q_{v}($ gross $)=8050 \mathrm{cal} \mathrm{g}^{-1}$
$q_{v}($ gross $)=33681 \mathrm{~J} \mathrm{~g}^{1}$

COAL, ANTHRACITE; solid; Ultimate analysis of combustible, ash free and moisture free: carbon, $94.39 \%$; hydrogen, $1.77 \%$; oxygen, $2.13 \%$; nitorgen, $0.71 \%$; sulfur, $1.00 \%$; proximate analysis: ash, $7.83 \%$; moisture, $2.80 \%$; volatile matter, $1.3 \%$; loss on air drying, $1.5 \%$.

gross heat of combustion: assume values refer to room temperature.

ash free and moisture free

$q_{v}($ gross $)=8273 \mathrm{cal} \mathrm{g}^{-1}$
$q_{v}($ gross $)=34616 \mathrm{~J} \mathrm{~g}^{1}$

as received

$q_{v}($ gross $)=7393 \mathrm{cal} \mathrm{g}^{-1}$
$q_{v}($ gross $)=30931 \mathrm{~J} \mathrm{~g}^{-1}$

$[47,48]$

COAI. SEMI-ANTHRACITE; solid; UTtimate analysis of combustible, ash free and moisture free: carbon, $92.15 \%$; hydrogen, $3.76 \%$; oxygen, $2.17 \%$; nitrogen, $1.18 \%$; sulfur, $0.74 \%$; proximate analysis: ash, $11.50 \%$; moisture, $3.38 \%$; volatile matter, $10.0 \%$; loss on air drying, $2.6 \%$.

gross heat of combustion: assume values refer to room temperature.

ash free and moisture free

$q_{v}($ gross $)=8593 \mathrm{cal} \mathrm{g}^{-1}$
$q_{v}($ gross $)=35953 \mathrm{~J} \mathrm{~g}^{1}$

as received

$q_{v}($ gruss $)=7314 \mathrm{cal} \mathrm{g}^{-1}$
$q_{v}($ gross $)=30601 \mathrm{~J} \mathrm{~g}^{1}$ 
COAL, BITUMINOUS (HIGH GRADE); solid; Ultimate analysis of combustible, ash free and moisture free: carbon, $85.09 \%$; hydrogen, $4.99 \%$; oxygen, $6.99 \%$; nitrogen, $1.80 \%$; sulfur, 1.13\%; proximate analysis: ash, $2.79 \%$; moisture, $2.18 \%$; volatile matter, $33.4 \%$; loss on air drying, $1.0 \%$.

gross heat of combustion: assume values refer to room temperature.

ash free and moisture free

$q_{v}$ (gross) $=8667 \mathrm{cal} \mathrm{g}^{-1}$
$q_{v}($ gross $)=36262 \mathrm{~J} \mathrm{~g}^{1}$

as received

$q_{v}($ gross $)=8237 \mathrm{cal} \mathrm{g}^{-1}$
$q_{v}($ gross $)=34462 \mathrm{Jg}^{1}$

$[47,48]$

COAL, BITUMINOUS (MEDIUM GRADE); solid; (sample 1) Ultimate analysis of combustible, ash free and moisture free: carbon, $82.08 \%$; hydrogen, $5.40 \%$; oxygen, $5.98 \%$; nitrogen, $1.32 \%$; sulfur, $5.22 \%$; proximate analysis: ash, $12.97 \%$; moisture, $4.99 \%$; volatile matter, 39.8\%; loss on air drying, 1.3\%; (sample 2) ultimate analysis of combustible, ash free and moisture free: carbon, $76.55 \%$; hydrogen, $5.26 \%$; oxygen, $11.83 \%$; nitrogen, $1.40 \%$; sulfur, $4.96 \%$; proximate analysis: ash, $23.38 \%$; moisture, $17.30 \%$; volatile matter, $44.6 \%$; loss on air drying, $15.2 \%$.

gross heat of combustion: assume values refer to room temperature.

sample 1, ash free and moisture free

$q_{v}$ (gross) $=8296 \mathrm{cal} \mathrm{g}^{-1}$
$q_{v}$ (gross) $=34709 \mathrm{~J} \mathrm{~g}^{1}$

sample 1, as received

$q_{v}($ gross $)=6806 \mathrm{cal} \mathrm{g}^{-1}$
$q_{v}($ gross $)=28475 \mathrm{~J} \mathrm{~g}^{1}$

sample 2, ash free and moisture free

$q_{v}($ gross $)=7723 \mathrm{cal} \mathrm{g}^{-1}$
$q_{v}($ gross $)=32313 \mathrm{~J} \mathrm{~g}^{-1}$

sample 2, as received

$q_{v}($ gross $)=4581 \mathrm{cal} \mathrm{g}^{-1}$
$q_{v}($ gross $)=19166 \mathrm{~J} \mathrm{~g}^{1}$ 
COAL, BITUHINOUS (LOW GRADE); solid; Ultimate analysis of conibustible, ash iree and muisture free: carbon, $76.11 \%$; hydrogen, $4.03 \%$; oxygen, $16.14 \%$; nitrogen, $0.84 \%$; sulfur, $2.88 \%$; proximate analysis: ash, $26.88 \%$; moisture, $10.88 \%$; volatile illutter, $32.6 \%$; loss on air drying, $5.4 \%$.

gross heat of combustion: assume values refer to room temperature.

ash free and moisture free

$q_{v}($ gross $)=6915 \mathrm{cal} \mathrm{g}^{-1}$
$q_{v}($ gross $)=28931 \mathrm{~J} \mathrm{~g}^{1}$

as received

$q_{v}($ gross $)=4304 \mathrm{cal} \mathrm{g}^{-1}$
$q_{v}($ gross $)=18008 \mathrm{jg}^{2}$

$[47,48]$

COAL, SEMI-BITUMINOUS; solid; Ultimate analysis of combustible, ash free and moisture

free: carbon, $89.79 \%$; hydrogen, $4.76 \%$; oxygen, $2.47 \%$; nitrogen, $2.00 \%$; sulfur, $0.98 \%$; proximate analysis: ash, $6.80 \%$; moisture, $2.60 \%$; volatile matter, $17.5 \%$; loss on air drying, $2.0 \%$.

gross heat of combustion: assume values refer to room temperature.

ash free and moisture free

$q_{v}($ gross $)=8811 \mathrm{cal} \mathrm{g}^{-1}$
$q_{v}($ gross $)=36867 \mathrm{Jg}^{1}$

as received

$q_{v}($ gross $)=7983 \mathrm{cal} \mathrm{g}^{-1}$
$q_{v}($ gross $)=33401 \mathrm{~J} \mathrm{~g}^{1}$

$[47,48]$

COAL, SUB-BITUMINCUS; solid; Ultimate analys is of combustible, ash free and moisture

free; carbon, $69.17 \%$; hydrogen, $4.46 \%$; oxygen, $24.35 \%$; nitrogen, $1.66 \%$; sulfur, $0.36 \%$; proximate analysis: ash, $3.71 \%$; moisture, $18.41 \%$; volatile matter, $44.3 \%$; loss on air drying, $10.0 \%$.

gross heat of combustion: assume values refer to room temperature.

ash free and moisture free

$q_{v}($ gross $)=6516 \mathrm{cal} \mathrm{g}^{-1}$
$q_{v}($ gross $)=27265 \mathrm{~J} \mathrm{~g}^{1}$

as received

$q_{v}($ gross $)=5076 \mathrm{cal} \mathrm{g}^{-1}$
$q_{v}($ gross $)=21236 \mathrm{~J} \mathrm{~g}^{-1}$

$[47,48]$ 
COAL, CANNEL; solid; Ultimate analysis of combustible, ash free and moisture free: carbon, 82.59\%; hydrogen, $7.13 \%$; oxygen, $7.57 \%$; nitrogen, $1.33 \%$; sulfur, $1.38 \%$; pruximate analysis: ash, $10.49 \%$; moisture, $2.36 \%$; volatile matter, $55.5 \%$; loss on air drying, $1.5 \%$.

gross heat of combustion: assume values refer to room temperature.

ash free and moisture free

$q_{v}($ gross $)=8784 \mathrm{cal} \mathrm{g}^{-1}$
$q_{v}($ gross $)=36751 \mathrm{Jg}^{1}$

as received

$q_{v}($ gross $)=7655 \mathrm{cal} \mathrm{g}^{-1}$
$q_{v}($ gross $)=32029 \mathrm{Jg}^{1}$

$[47,48]$

COKE; solid; Produced from the destructive distillation of gas-coal in gas retorts;

(sample 1, as received): fixed carbon, $59.56 \%$; sulfur, $0.31 \%$; moisture, $22.30 \%$; ash, $17.56 \%$; volatile matter, $0.58 \%$; (sample 1, dry): fixed carbon, $76.65 \%$; sulfur, $0.40 \%$; ash, $22.60 \%$; volatile matter, $0.75 \%$; (sample 2 , as received): fixed carbon, $67.89 \%$; sulfur, $0.51 \%$; ash, $4.45 \%$; moisture, $26.80 \%$; volatile matter, $0.86 \%$; (sample 2, dry): fixed carbon, $92.75 \%$; sulfur, $0.70 \%$; ash, $6.08 \%$; volatile matter, 1.17\%; (dry represents sample as drawn from retort before quenching).

gross heat of combustion: assume values refer to room temperature.

sample 1, as received

$q_{v}($ gross $)=4826 \mathrm{cal} \mathrm{g}^{-1}$
$q_{v}($ gross $)=20192 \mathrm{~J} \mathrm{~g}^{-1}$

sample 1, dry

$q_{v}($ gross $)=6211 \mathrm{cal} \mathrm{g}^{-1}$
$q_{v}($ gross $)=25988 \mathrm{~J} \mathrm{~g}^{1}$

sample 2, as received

$q_{v}($ gross $)=5612 \mathrm{cal} \mathrm{g}^{-1}$
$q_{v}($ gross $)=23479 \mathrm{Jg}^{-1}$

sample 2, dry

$q_{v}($ gross $)=7666 \mathrm{cal} \mathrm{g}^{-1}$
$q_{v}($ gross $)=32076 \mathrm{~J} \mathrm{~g}^{1}$

$[47,50]$ 
COKE; solid; Produced from the destructive distillation of bituminous coal in beehive ovens; (sample 1): fixed carbon, $91.43 \%$; volatile carbon, $1.27 \%$; sulfur, 0.51 ; ; ash, 6.09\%; (sample 2): fixed carbon, $79.83 \%$; volatile carbon, $1.05 \%$; sulfur, $2.13 \%$; ash, $15.75 \%$; water, $1.22 \%$.

gross heat of combustion: assume values refer to roun temperatures; col rected for moisture and ash content.

sample l

$q_{v}($ gross $)=8011 \mathrm{cal} \mathrm{g}^{-1}$
$q_{v}($ gross $)=33520 \mathrm{~J} \mathrm{~g}_{1}$

sample 2

$q_{v}($ gross $)=7718 \mathrm{cal} \mathrm{g}^{-1}$
$q_{v}($ gross $)=32292 \mathrm{~J} \mathrm{~g}^{1}$

COKE; solid; Produced from the destructive distillation to red heat of petroleum; fixed carbon, $98.05 \%$; volatile carbon, $0.50 \%$.

jross heat of combustion: assume values refer to room temperature.

$q_{v}(\operatorname{gross})=8063 \mathrm{cal} \mathrm{g}^{-1}$
$u_{v}($ gross $)=33734 \mathrm{~J} \mathrm{~g}^{2}$

COKE BREEZE; solid; Ultimate analysis: carbon, $94.79 \%$; hydrogen, $1.51 \%$; oxygen, $1.48 \%$; nitrogen, 1.19\%; sulfur, 1:03\%; proximate analysis: ash, $12.05 \%$; moisture, $10.77 \%$; volatile matter, $4.92 \%$; loss on air drying, $10.1 \%$.

gross heat of combustion: assume values refer to room temperature.

ash tree and moisture free

$\eta_{v}($ gross $)=8038 \mathrm{cal} \mathrm{g}^{-1}$
$\eta_{v}($ gross $)=33632 \mathrm{~J} \mathrm{~g}^{2}$

as received

$q_{v}($ gross $)=6204 \mathrm{cal} \mathrm{g}^{-1}$
$q_{v}($ gross $)=25958 \mathrm{~J} \mathrm{~g}^{-1}$

$[47,51]$

IJUWIILERM A; liquid; (trademark c) Dow Chemical Co.); a mixture of diphenyl and diphenyi oxide.

spec 1 fic: heat:

$30-240^{\circ} \mathrm{C}$

$\left.C p(c a l\}^{-1} K^{-1}\right)=0.167+7.40 \times 10^{-4} T^{\top}$
$C p\left(d g^{-1} K^{-1}\right)=0.699+3.096 \times 10^{-3} T$ 


\section{EXPLOSIVES}

AMATOL; solid; an explosive mixture of ammonium nitrate (AN) and trinitrotoluene (TNT). Density of cast material, $9 \mathrm{~cm}^{-3}: 80 / 20$ AN/TNT, $1.46 ; 60 / 40$ AN/TNT, $1.60 ; 50 / 5$, AN/TNT, 1.59 .

gross heat of combustion: $25{ }^{\circ} \mathrm{C}$; calculated from the composition of the mixture.

80/20 AN/TNT

$q_{v}($ gross $)=1219 \mathrm{cal} \mathrm{g}^{-1}$
$q_{v}($ gross $)=5100 \mathrm{Jg}^{-1}$

$60 / 40$ AN/TNT

$q_{v}($ gross $)=1811 \mathrm{cal}^{-\mathrm{g}^{-1}}$
$q_{v}($ gross $)=7577 \mathrm{Jg}^{-1}$

50/50 AN/TNT

$q_{v}($ gross $)=2107 \mathrm{cal}^{-g^{-1}}$
$q_{v}($ gross $)=8816 \mathrm{Jg}^{-1}$

$[77,132,134]$

specific heat: for the temperature range $20^{\circ}$ to $80^{\circ} \mathrm{C}$ for $50 / 50$ AN/TNT mixture.

$c_{p}=0.383 \mathrm{cal}^{-1} \mathrm{~g}^{-1}$
$c_{p}=1.602 \mathrm{~J} \mathrm{~g}^{-1} K^{-1}$

net heat of explosion: $25{ }^{\circ} \mathrm{C}$; calculated from composition of the mixture; assumed decomposition reactions of AN and TNT are given below; all products are gaseous unless otherwise indicated.

$80 / 20$ AN/TNT

$q_{v}($ net $)=499 \mathrm{cal} \mathrm{g}^{-1}$
$q_{v}($ net $)=2088 \mathrm{Jg}^{-1}$

$60 / 40$ AN/TNT

$q_{v}($ net $)=646 \mathrm{cal} \mathrm{g}^{-1}$
$q_{v}($ net $)=2703 \mathrm{Jg}^{-1}$

50/50 AN/TNT

$q_{v}$ (net) $=719 \mathrm{cal} \mathrm{g}^{-1}$
$q_{v}$ (net) $=3008 \mathrm{~J} \mathrm{~g}^{1}$

$[77,132,133,134]$

decomposition reactions:

(AN)(solid) $=2 \mathrm{H}_{2} \mathrm{O}+\mathrm{N}_{2}+0.5 \mathrm{O}_{2}$

(TNT)(solid) $=1.25 \mathrm{CO}_{2}+2.0 \mathrm{CO}+1.5 \mathrm{H}_{2} \mathrm{O}+1.25 \mathrm{~N}_{2}+0.5 \mathrm{NH}_{3}+0.5 \mathrm{H}_{2}$

$+3.75 \mathrm{C}($ solid)

explosion temperature after 5 seconds:

$80 / 20$ AN/TNT, $280{ }^{\circ} \mathrm{C}$

$60 / 40$ AN/TNT, $270{ }^{\circ} \mathrm{C}$

$50 / 50$ AN/TNT, $265{ }^{\circ} \mathrm{C}$ 


\section{EXPLOSIVES}

BARATOL; solid; barium nitrate, $75 \%$; trinitrntoiuere (INT), 25:"

heat of fusion:

$L_{f}=2.8 \mathrm{cal} \mathrm{g}^{-1}$
$L_{f}=11.7 \mathrm{Jg}^{2}$

specific heat:

$\begin{array}{ccc}\text { temperature }\left({ }^{\circ} \mathrm{C}\right) & \left.C p(\mathrm{ca}) \mathrm{g}^{-1} \mathrm{~K}^{-1}\right) & \left.C_{p}(.) \mathrm{y}^{-1} \mathrm{~K}^{-1}\right) \\ -75 & 0.152 & 0.636 \\ 0 & 0.147 & 0.615 \\ 25 & 0.180 & 0.753 \\ 50 & 0.229 & 0.958 \\ 75 & 0.280 & 1.172 \\ 85 & 0.213 & 0.891 \\ 90 & 0.201 & 0.841 \\ 100 & 0.171 & 0.715\end{array}$

[77]

BARONAL; solid; barium nitrate, 50\%; trinitrotoluene (TNT), 35\%; aluminum, 15\%; density $2.32 \mathrm{~g} \mathrm{~cm}^{-3}$.

gross heat of combustion: $25{ }^{\circ} \mathrm{C}$; calculated from the composition of the mixture.

$q_{v}($ gross $)=2367 \mathrm{cal}^{-\mathrm{g}^{-1}}$
$q_{v}($ gross $)=9904 \mathrm{~J} \mathrm{~g}^{-1^{2}}$

$[77,132,133,134]$

!lel leat of explosion: reaction products unspecified.

$q_{v}($ net $)=1135 \mathrm{cal}^{-1} \mathrm{~g}^{-1}$
$q_{v}($ net $)=4749 \mathrm{~J} \mathrm{~g}^{-1}$

explosion temperature after 5 seconds: Ignites at $345{ }^{\circ} \mathrm{C}$.

WIACK POWDER; solid; potassium nitrate, $74 \%$; sulfur, $10.4 \%$; charcoal, $15.6 \%$.

net. heat of explosion: $25^{\circ} \mathrm{C}$; calculated from composition of mixture; assumed decomposition reaction below.

uv $_{v}($ net $)=649 \mathrm{cal}^{-1}$
$\eta_{v}($ net $)=2715 \mathrm{Jg}^{1}$

$[77,132,133,134]$

explosion temperature after 5 seconds: I gnites at $427^{\circ} \mathrm{C}$.

decomposition reaction:

$K N()_{3}($ solid $)+0.25 \mathrm{~S}($ solid $)+C($ solid $)=0.25 \mathrm{~K}_{2} \mathrm{SO}_{3}($ solid $)+0.25 \mathrm{~K}_{2} \mathrm{CO}_{3}($ solid $)+$

$0.75 \mathrm{CO}_{2}$ (gas) 


\section{EXPLOSIVES}

BLASTING GELATINE; solid; gelatine with about $5 \%$ of an oxidizer, such as ammonium nitrate, potassium nitrate, or potassium chlorate, added.

net heat of combustion: unclear whether a gaseous oxidizer, such as air or oxygen, took part in combustion in addition to oxidizer in gelatine; assume values refer to room temperature, and one atm. pressure, and gaseous products.

$q_{p}($ net $)=570 \mathrm{cal} \mathrm{g}^{-1}$
$q_{p}($ net $)=2385 \mathrm{~J} \mathrm{~g}^{1}$

CELLULOSE NITRATE; solid; cellulose does not nitrate in a stoichiometric manner; the degree of nitration is measured by the percent of nitrogen in the ester.

mononitrocellulose: $\left[\mathrm{C}_{6} \mathrm{H}_{9} \mathrm{NO}_{7}\right]_{x} ; 6.76 \%$ nitrogen

dinitrocellulose: $\left[\mathrm{C}_{6} \mathrm{H}_{8} \mathrm{~N}_{2} \mathrm{O} 9\right]_{x} ; 11.11 \%$ nitrogen

trinitrocellulose: $\left[\mathrm{C}_{6} \mathrm{H}_{7} \mathrm{~N}_{3} \mathrm{O}_{11}\right]_{x} ; 14.14 \%$ nitrogen

Cellulose Nitrate, Grade $B$ (Guncotton) $\mathrm{C}_{6} \mathrm{H}_{7}{ }_{26} \mathrm{~N}_{2} \quad 7_{4} \mathrm{O}_{10} 48$

Cellulose Nitrate, Grade C (Type I) $\mathrm{C}_{6} \mathrm{H}_{7.36} \mathrm{~N}_{2.670_{10.27}}$

Cellulose Nitrate, Grade C (Type II) $\mathrm{C}_{6} \mathrm{H}_{7.33} \mathrm{~N}_{2.67} \mathrm{O}_{10.34}$

Cellulose Nitrate, Grade D (Pyroxylin) $\mathrm{C}_{6} \mathrm{H}_{7} .68 \mathrm{~N}_{2.32} \mathrm{O}_{0.64}$

gross heat of combustion: at $30{ }^{\circ} \mathrm{C}$ for $[0.115 \leq N \leq 0.135] ; N=$ wt. fraction nitrogen.

$q_{v}($ gross $)=4176.0-14126 \mathrm{~N} \mathrm{cal} \mathrm{g}^{-1}$
$q_{v}($ gross $)=17472.4-59103 \mathrm{~N} \mathrm{~J} \mathrm{~g}^{1}$

specific heat:

298 - $390 k$

Grade B (Guncotton) $13.4 \%$ nitrogen

$C_{p}\left(c_{1} g^{-1} K^{-1}\right)=0.0184-7.64 \times 10^{-4} T$

$C p\left(\mathrm{Jg}^{-1} \mathrm{~K}^{-1}\right)=0.0770-3.197 \times 10^{-3} \mathrm{~T}$

Grade C (Type I) $13.1 \%$ nitrogen

$C p\left(\right.$ cal $\left.g^{-1} K^{-1}\right)=0.0201-7.86 \times 10^{-4} T$

$\mathrm{Cp}\left(\mathrm{J} \mathrm{g}^{-1} \mathrm{~K}^{-1}\right)=0.0841-3.289 \times 10^{-3} \mathrm{~T}$

Grade C (Type II) $13.2 \%$ nitrogen

$C p\left(c a l g^{-1} K^{-1}\right)=0.0241-7.91 \times 10^{-4} T$

$C p\left(\mathrm{Jg}^{-9} \mathrm{~K}^{-1}\right)=0.1008-3.310 \times 10^{-3} \mathrm{~T}$

Grade D (pyroxylin) $12.2 \%$ nitrogen

$\operatorname{Cp}\left(\mathrm{cal} \mathrm{g}^{-1} \mathrm{~K}^{-1}\right)=0.0256-8.17 \times 10^{-4} \mathrm{~T}^{-1}$
$\operatorname{Cp}\left(\mathrm{Jg} \mathrm{g}^{-1}\right)=0.1071-3.418 \times 10^{-3} \mathrm{~T}^{-1}$ 


\section{EXPLOSIVES}

COMPOSITION B; solid; cyclotrimethylenetrinitramine (RDX), 60\%; trinitrotoluene (INT), $40 \%$; wax alcohol $27 \%$; density, cast, $1.65 \mathrm{~g} \mathrm{~cm}^{-3}$.

gross heat of combustion: assume values refer to room temperature.

$q_{v}($ gross $)=2790 \mathrm{cal} \mathrm{g}^{-1}$
$q_{v}($ gross $)=11673 \mathrm{~J} \mathrm{~g}^{2}$

net heat of explasion: assume values refer to room temperature; reaction producis unspecified.

$q_{v}($ net $)=1240 \mathrm{cal}^{-g^{-1}}$
$q_{v}($ net $)=5188 \mathrm{~J} \mathrm{~g}^{-1}$

heat of fuston: at $78-80^{\circ} \mathrm{C}$.

$L_{f}=8.0 \mathrm{cal} \mathrm{g}^{-1}$
$L_{f}=33.5 \mathrm{Jg}^{-1}$

[77]

explosion temperature after 5 seconds: Decomposes at $278^{\circ} \mathrm{C}$.

specific heat:

$\begin{array}{ccc}\text { temperature, }{ }^{\circ} \mathrm{C} & C p\left(\mathrm{cal} \mathrm{g}^{-1} \mathrm{~K}^{-1}\right) & \mathrm{Cp}\left(\mathrm{J} \mathrm{g}^{-1} \mathrm{~K}^{-1}\right) \\ -75 & 0.235 & 0.983 \\ 0 & 0.220 & 0.920 \\ 25 & 0.254 & 1.063 \\ 50 & 0.305 & 1.276 \\ 75 & 0.376 & 1.573 \\ 85 & 0.354 & 1.481 \\ 90 & 0.341 & 1.427 \\ 100 & 0.312 & 1.305\end{array}$

[77] 
CYCLOTOL; solid; an explosive mixture of cyclotrimethylenetrinitramine (RDX) and trinitrotoluene (TNT); density of cast material $\left(\mathrm{g} \mathrm{cm}^{-3}\right)$ : 75/25 ROX/TNT, 1.71; 70/80 RDX/TNT, $1.71 ; 65 / 35$ RDX/TNT, $1.75 ; 60 / 40$ RDX/TNT, 1.68 .

gross heat of combustion: $25^{\circ} \mathrm{C}$; calculated from the composition of the nixture.

$75 / 25$ RDX/TNT

$q_{v}($ gross $)=2609 \mathrm{cal} \mathrm{g}^{-1}$
$q_{v}($ gross $)=10916 \mathrm{~J} \mathrm{~g}^{1}$

70/30 RDX/TNT

$q_{v}($ gross $)=2674 \mathrm{cal} \mathrm{g}^{-1}$
$q_{v}($ gross $)=11188 \mathrm{~J} \mathrm{~g}^{1}$

$65 / 35$ RDX/TNT

$q_{v}($ gross $)=2739 \mathrm{cal} \mathrm{g}^{-1}$
$q_{v}($ gross $)=11460 \mathrm{~J} \mathrm{~g}^{2}$

60/40 RDX/TNT

$q_{v}($ gross $)=2804 \mathrm{cal} \mathrm{g}^{-1}$
$q_{v}($ gross $)=11732 \mathrm{~J} \mathrm{~g}^{2}$

$[77,132,138]$

net heat of explosion: assume values refer to room temperature; calculated from the composition of the mixture; reaction products unspecified.

75/25 RDX/TNT

$q_{v}$ (net) $=1231 \mathrm{cal}^{-g^{-1}}$
$q_{v}$ (net) $=5150 \mathrm{~J} \mathrm{~g}^{-1}$

70/30 RDX/TNT

$q_{v}($ net $)=1222 \mathrm{cal} \mathrm{g}^{-1}$
$q_{v}($ net $)=5113 \mathrm{~J} \mathrm{~g}^{-1}$

65/35 RDX/TNT

$q_{v}($ net $)=1212 \mathrm{cal}^{-g^{-1}}$
$q_{v}($ net $)=5071 \mathrm{Jg}^{-1}$

60/40 RDX/TNT

$q_{v}$ (net) $=1202 \mathrm{cal} \mathrm{g}^{-1}$

$q_{v}^{v}($ net $)=5029 \mathrm{~J} \mathrm{~g}^{-1}$ 
specific heat: $75 / 25$ RDX/TNT.

$\begin{array}{ccc}\text { temperature, }{ }^{\circ} \mathrm{C} & \left.C p(c a) g^{-1} \mathrm{~K}^{-1}\right) & C p\left(\mathrm{~J} \mathrm{~g}^{-1} \mathrm{~K}^{-1}\right) \\ -75 & 0.220 & 0.920 \\ 0 & 0.225 & 0.941 \\ 25 & 0.254 & 1.063 \\ 50 & 0.296 & 1.238 \\ 75 & 0.352 & 1.473 \\ 85 & 0.325 & 1.360 \\ 90 & 0.332 & 1.389 \\ 100 & 0.351 & 1.469\end{array}$

heat of fusion: $75 / 25$ RDX/TNT.

$L_{f}=5.0 \mathrm{cal} \mathrm{g}^{-1} \quad(T=?)$
$L_{f}=20.9 \mathrm{~J} \mathrm{~g}^{2}$

explosion temperature after 5 seconds:

70/30 RDX/TNT, decomposes $265^{\circ} \mathrm{C}$.

$65 / 35$ RDX/TNT, decomposes $270{ }^{\circ} \mathrm{C}$.

$60 / 40$ RDX/TNT, decomposes $280^{\circ} \mathrm{C}$.

DBX; solid; annonium nitrate (AN), 21\% cyclotrimethylenetrinitramine (RDX), $21 \%$; trinitrotoluene (TNT), $40 \%$; aluminum, $18 \%$; density $1.68 \mathrm{~g} \mathrm{~cm}^{-3}$.

gross heat of combustion: assume values refer to room temperature; calculated from the composition of the mixture.

$q_{v}($ gross $)=3379 \mathrm{cal} \mathrm{g}^{-1}$
$q_{v}($ gross $)=14138 \mathrm{~J} \mathrm{~g}^{2}$

$[77,132,134,136,138]$

net heat of explosion: assume values refer to room temperature; reaction products unspecified.

$q_{v}$ (net) $=1700 \mathrm{cal}^{-\mathrm{g}^{-1}}$
$q_{v}($ net $)=7113 \mathrm{Jg}^{-1}$

specific hedt: $\quad-5^{\circ} \mathrm{C}$

$C p\left(\right.$ cal $\left.g^{-1} k^{-1}\right)=0.25$
$C p\left(.3 y^{-1} k^{-1}\right)=1.05$

explosion lemperature after 5 seconds: Ignites at $400{ }^{\circ} \mathrm{C}$. 


\section{EXPLOSIVES}

H-б; solid, cyclotrimethylenetrinitramine (RDX), 45\%; trinitrotoluene (TNT), 30\%; aluminum, $20 \%$; D-2 wax, $5 \%$; calcium chloride added, $0.5 \%$; density; cast, $1.74 \mathrm{~g} \mathrm{~cm}^{-3}$.

gross heat of combustion: assume values refer to room temperature.

$q_{v}($ gross $)=3972 \mathrm{cal} \mathrm{g}^{-1}$
$q_{v}($ gross $)=16619 \mathrm{~J} \mathrm{~g}^{1}$

net heat of explosion: assume values refer to room temperature; reaction products unspecified.

$$
\begin{aligned}
& q_{v} \text { (net) }=923 \mathrm{cal} \mathrm{g}^{-1} \\
& q_{v} \text { (net) }=3862 \mathrm{~J} \mathrm{~g}^{1} \\
& \text { heat of fusion: at } 78{ }^{\circ} \mathrm{C} . \\
& L_{f}=10.25 \mathrm{cal} \mathrm{g}^{-1} \\
& L_{f}=42.89 \mathrm{~J} \mathrm{~g}^{-1}
\end{aligned}
$$

specific heat:

$$
\begin{aligned}
& \mathrm{Cp}\left(\mathrm{cal} \mathrm{g}^{-1} \mathrm{~K}^{-1}\right)=0.269\left(30^{\circ} \mathrm{C}\right) \\
& C p\left(\mathrm{~J} \mathrm{~g}^{-1} \mathrm{~K}^{-1}\right)=1.125\left(30^{\circ} \mathrm{C}\right) \\
& \mathrm{Cp}\left(\mathrm{cal}-\mathrm{g}^{-1} \mathrm{~K}^{-1}\right)=0.268\left(50^{\circ} \mathrm{C}\right) \\
& C p\left(\mathrm{~J} \mathrm{~g}^{-1} \mathrm{~K}^{-1}\right)=1.121\left(50^{\circ} \mathrm{C}\right)
\end{aligned}
$$

explosion temperature after 5 seconds: $610^{\circ} \mathrm{C}$ (minimum) 


\section{EXPLOSIVES}

HBX-1; solid; cyclotrimethylenetrinitramine (RDX), 40\%; trinitrotoluene (TNT), 3r.; aluminum, $17 \%$; D-2 wax, $5 \%$; calcium chloride added, $0.5 \%$; density, cast, 1.72 $\mathrm{g} \mathrm{cm}^{-3}$.

gross heat of combustion: assume values refer to room temperature.

$q_{v}($ gross $)=3882 \mathrm{cal} \mathrm{g}^{-1}$

$q_{v}^{v}($ gross $)=16242 \mathrm{Jg}^{2}$

net heat of explosion: assume values refer to room temperature; reaction products unspecified.

$q_{v}($ net $)=919 \mathrm{cal} \mathrm{g}^{-1}$
$q_{v}($ net $)=3845 \mathrm{Jg}^{1}$

heat of fusion: at $78^{\circ} \mathrm{C}$.

$L_{f}=9.25 \mathrm{cal} \mathrm{g}^{-1}$
$L_{f}=38.70 \mathrm{~J} \mathrm{~g}^{-1}$

specific heat:

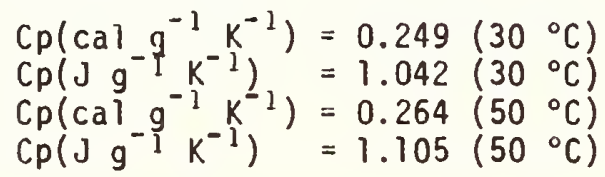

explosion temperature after 5 seconds: $480{ }^{\circ} \mathrm{C}$ 


\section{EXPLOSIVES}

HBX-3; solid; cyclotrimethylenetrinitramine (RDX), 31\%; trinitrotoluene (TNT), 29:; aluminum, $35 \%$; D-2 wax, $5 \%$; calcium chloride added, $0.5 \%$; density, cast, 1.84 $\mathrm{g} \mathrm{\textrm {cm } ^ { - 3 }}$.

gross heat of combustion: assume values refer to room temperature.

$q_{v}$ (gross) $=4495 \mathrm{cal} \mathrm{g}^{-1}$
$q_{v}($ gross $)=18807 \mathrm{~J} \mathrm{~g}^{1}$

net heat of explosion: assume values refer to room temperature; reaction products unspecified.

$q_{v}($ net $)=877 \mathrm{cal} \mathrm{g}^{-1}$
$q_{v}($ net $)=3669 \mathrm{~J} \mathrm{~g}^{-1}$

heat of fusion:

$L_{f}=9.30 \mathrm{cal}_{f} \mathrm{~g}^{-1}$
$L_{f}=38.9 \mathrm{~J} \mathrm{~g}^{-I^{2}}$

specific heat:

$30-50{ }^{\circ} \mathrm{C}$.

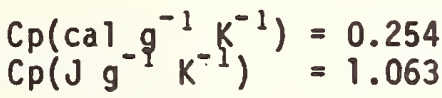

explosion temperatures after 5 seconds: $500{ }^{\circ} \mathrm{C}$.

HEX-24; solid; potassium perchlorate (17 microns), 32\%; aluminum, a tomized, (20 microns), 48\%; cyclotrimethyl enetrinitramine (RDX) (through 325 mesh), 16\%; asphal tum (through 100 mesh), $4 \%$; density, $1.39 \mathrm{~g} \mathrm{~cm}^{-3}$.

gross heat of combustion: assume values refer to room temperature.

$q_{v}($ gross $)=4197 \mathrm{cal} \mathrm{g}^{-1}$
$q_{v}$ (gross) $=17560 \mathrm{~J} \mathrm{~g}^{1}$

net heat of explosion: assume values refer to room temperature; reaction products unspecified.

$q_{v}($ net $)=1858 \mathrm{cal} \mathrm{g}^{-1}$
$q_{v}($ net $)=7774 \mathrm{Jg}^{-1}$

explosion temperature after 5 seconds: $520{ }^{\circ} \mathrm{C}$. 


\section{EXPLOSIVES}

HEX-48; solid; potassium perchlorate, (17 microns), $32 \%$; aluminum, flaked

(1 micron), 48\%; cyclotrimethylenetrinitrailine (RDX) (through 325 mesh), 16\%; asphal tum (through 100 mesh). $4 \%$; density, $0.69 \mathrm{~g} \mathrm{~cm}^{-3}$.

gross heat of combustion: assume values refer to room temperature.

$q_{v}($ gross $)=4119 \mathrm{cal} \mathrm{g}^{-1}$
$q_{v}($ gross $)=17234 \mathrm{~J} \mathrm{~g}^{1}$

net heat of explosion: assume values refer to room temperature; reaction products unspecified.

$q_{v}($ net $)=1735 \mathrm{cal}^{-\mathrm{g}^{-1}}$
$q_{v}$ (net) $=7259 \mathrm{~J} \mathrm{~g}^{-1^{2}}$

explosion temperature after 5 seconds: $545^{\circ} \mathrm{C}$.

HTA-3; solid; cyclotetramethyl enetetranitramine (HMX), 49\%; trinitrotoluene (TNT), 29\%; aluminum, $22 \%$; density, cast, $1.90 \mathrm{~g} \mathrm{~cm}^{-3}$.

gross heat of combustion: $25{ }^{\circ} \mathrm{C}$; calculated from the composition of the mixture

$q_{v}($ gross $)=3785 \mathrm{cal} \mathrm{g}^{-1}$
$q_{v}($ gross $)=15836 \mathrm{Jg}^{1}$

$[74,132,134,138]$

net heat of explosion: assume values refer to room temperature; reaction products unspecified.

$q_{v}($ net $)=1190 \mathrm{cal} \mathrm{g}^{-1}$
$q_{v}($ net $)=4979 \mathrm{~J} \mathrm{~g}^{-1}$

specific heat:

$32-74{ }^{\circ} \mathrm{C}$.

$\operatorname{Cp}\left(\mathrm{cal} \mathrm{g}^{-1} \mathrm{~K}^{-1}\right)=0.245$

$\left.C p()^{-1} \mathrm{~K}^{-1}\right)=1.025$

[77]

explosion temperature after 5 seconds: Flames erratically at $370^{\circ} \mathrm{C}$.

[77] 


\section{EXPLOSIVES}

MoX-1; solid; ammonium perchlorate, $35 \%$; atomized aluminum, $26.2 \%$; atomized magnesium, $26.2 \%$; tetry $1,9.7 \%$; calcium stearate, $1.9 \%$; artificial graphite, $1.0 \%$; densitj, pressed, $2.0 \mathrm{~g} \mathrm{~cm}^{-3}$.

gross heat of combustion: assume values refer to room temperature.

$q_{v}($ gross $)=4087 \mathrm{cal} \mathrm{g}^{-1}$
$q_{v}($ gross $)=17100 \mathrm{~J} \mathrm{~g}^{1}$

net heat of explosion: assume values refer to room temperature; reaction products unspecified.

$q_{v}$ (net) $=2087 \mathrm{cal}^{-\mathrm{g}^{-1}}$
$\mathrm{q}_{v}$ (net) $=8732 \mathrm{~J} \mathrm{~g}^{-1}$

explosion temperature after 5 seconds: $285^{\circ} \mathrm{C}$

MOX-2B; solid; ammonium perchlorate, 35\%; atomized aluminum, $52.4 \%$; cyclotrimethylentetrinitramine (RDX), 5.8\%; trinitrotoluene (TNT), 3.9\%; calcium stereate, $1.9 \%$; arti. ficial graphite, $1.0 \%$; density, pressed, $2.0 \mathrm{~g} \mathrm{~cm}^{-3}$.

gross heat of combustion: assume values refer to room temperature.

$q_{v}($ gross $)=4484 \mathrm{cal} \mathrm{g}^{-1}$
$q_{v}($ gross $)=18761 \mathrm{~J} \mathrm{~g}^{1}$

net heat of explosion: assume values refer to room temperature; reaction products unspecified.

$q_{v}$ (net) $=1472 \mathrm{cal} \mathrm{g}^{-1}$
$q_{v}($ net $)=6159 \mathrm{Jg}^{-1}$

explosion temperature after 5 seconds: $375{ }^{\circ} \mathrm{C}$ 


\section{EXPLOSIVES}

MoX-3B; solid; potassium nitrate, $18 \%$; a tomized aluminum, $50 \%$; cyclotrimethylenetrinitramine (RDX), 29.1\%; wax, 0.9\%; trinitrotoluene (TNT), 2.0\%; artificial graphite, $1.0 \%$; calcium stearate $2.0 \%$ added; density, pressed, $2.0 \mathrm{~g} \mathrm{~cm}^{-3}$.

gross heat of combustion: assume values refer to room temperature.

$q_{v}($ gross $)=4331 \mathrm{cá}^{\prime} \mathrm{g}^{-1}$
$q_{v}($ gross $)=18121 \mathrm{~J} \mathrm{~g}^{1}$

net heat of explosion: assume values refer to room temperature; reaction products unspecified.

$q_{v}($ net $)=980 \mathrm{cal} \mathrm{g}^{-1}$
$q_{v}($ net $)=4100 \mathrm{~J} \mathrm{~g}^{-1}$

explosion temperature after 5 seconds: $540{ }^{\circ} \mathrm{C}$.

MOX-4B; solid; barium nitrate, 18\%; a tomized aluminum, 50\%; cyclotrimethylenetrinitramine (RDX), 29.1\%; wax, 0.9\%; trinitrotoluene (TNT), 2.0\%; calcium stearate, $2.0 \%$ added; artificial graphite, $1.0 \%$ added; density, pressed, $2.0 \mathrm{~g} \mathrm{~cm}^{-3}$. gross heat of combustion: assume values refer to room temperature.

$q_{v}($ gross $)=4392 \mathrm{cal} \mathrm{g}^{-1}$
$q_{v}($ gross $)=18376 \mathrm{~J} \mathrm{~g}^{1}$

net heat of explosion: assume values refer to room temperature; reaction products unspecified.

$q_{v}($ net $)=709 \mathrm{cal} \mathrm{g}^{-1}$
$q_{v}($ net $)=2966 \mathrm{~J} \mathrm{~g}^{1}$

explosion temperature after 5 seconds: $610^{\circ} \mathrm{C}$ 


\section{EXPLOSIVES}

MOX-6B; solid; atomized aluminum, $49.2 \%$; cupric oxide, $19.7 \%$; cyclotrimethylenetrinitramine $(R D X), 28.7 \%$; wax, $0.9 \%$; artificial graphite, $1.5 \%$.

gross heat of combustion: assume values refer to room temperature.

$q_{v}$ (gross) $=4293 \mathrm{cal} \mathrm{g}^{-1}$
$q_{v}($ gross $)=17962 \mathrm{~J} \mathrm{~g}^{1}$

net heat of explosion: assume values refer to room temperature; reaction products unspecified.

$q_{v}($ net $)=750 \mathrm{cal} \mathrm{g}^{-1}$
$q_{v}($ net $)=3138 \mathrm{~J} \mathrm{~g}_{1}$

explosion temperature after 5 seconds: $510^{\circ} \mathrm{C}$

PB-RDX; solid; cyclotrimethyl enetrinitramine (RDX), $90 \%$; unmodified polystyrene, $8.5 \%$; dioctyl phthalate, $1.5 \%$.

gross heat of combustion: assume values refer to room temperature.

$q_{v}($ gross $)=3027 \mathrm{cal} \mathrm{g}^{-1}$
$q_{v}($ gross $)=12665 \mathrm{~J} \mathrm{~g}^{-1}$

net heat of explosion: assume values refer to room temperature; reaction products unspecified.

$q_{v}$ (net) $=983 \mathrm{cal} \mathrm{g}^{-1}$
$q_{v}($ net $)=4113 \mathrm{Jg}^{-1}$

explosion temperature after 5 seconds: Smokes, $275^{\circ} \mathrm{C}$. 


\section{EXPLOSIVES}

MINOL-2; solid; ammonium nitrate (AN); $40 \%$; trinitrotoluene (TNT); $40 \%$; aluminum, $20 \%$; density, $1.65 \mathrm{~g} \mathrm{~cm}^{-3}$.

gross heat of combustion: $25^{\circ} \mathrm{C}$; calculated from the composition of the mixture.

$q_{v}($ gross $)=3166 \mathrm{cal} \mathrm{g}^{-1}$
$q_{v}($ gross $)=13247 \mathrm{Jg}^{-1}$

$[77,132,134]$

net heat of explosion: assume values refer to room temperature; reaction products unspecified.

$q_{v}($ net $)=1620 \mathrm{cal}^{-\mathrm{g}^{-1}}$
$q_{v}($ net $)=6778 \mathrm{~J} \mathrm{~g}^{-1}$

specific heat: at $-5{ }^{\circ} \mathrm{C}$

$C_{p}=0.30 \mathrm{cal}^{-g^{-1} \mathrm{~K}^{-1}}$
$C_{p}=1.26 \mathrm{~J} \mathrm{~g}^{-1} \mathrm{~K}^{-1}$

[77]

explosion temperature after 5 seconds: Ignites $435{ }^{\circ} \mathrm{C}$.

OCTOL; solid; an explosive mixture of cyclotetramethylenetetranitramine (HMX) and trinitrotoluene (TNT); density, cast, $70 / 30$ HMX/TNT, $1.80 \mathrm{~g} \mathrm{~cm}^{-3} ; 75 / 25$ HMX/TNT, $1.81 \mathrm{~g} \mathrm{~cm}^{-3}$.

gross heat of combustion: $25^{\circ} \mathrm{C}$; calculated from the composition of the mixture.

70/30 HMX/TNT

$q_{v}($ gross $)=2670 \mathrm{cal} \mathrm{g}^{-1}$
$q_{v}($ gross $)=11171 \mathrm{~J} \mathrm{~g}^{1}$

75/25 HMX/TNT

$q_{v}($ gross $)=2604 \mathrm{cal} \mathrm{g}^{-1}$
$q_{v}($ gross $)=10895 \mathrm{~J} \mathrm{~g}^{1}$

$[77,132,138]$ 


\section{EXPLOSIVES}

net heat of explosion: assume values refer to room temperature; reaction products unspecified.

70/30 HMX/TNT

$q_{v}($ net $)=1074 \mathrm{cal}^{-g^{-1}}$
$q_{v}($ net $)=4494 \mathrm{~J} \mathrm{~g}^{-1}$

$75 / 25$ HMX/TNT

$q_{y}$ (net) $=1131$ cal $g^{-1}$

$q_{v}$ (net) $=4732 \mathrm{~J} \mathrm{~g}^{-1}$

[77]

explosion temperature after 5 seconds:

70/30 HMX/TNT; Flames erratically, $335^{\circ} \mathrm{C}$

75/25 HMX/TNT; Flames erratically, $350^{\circ} \mathrm{C}$

heat of fusion:

76.9/23.1 HMX/TNT

$L_{f}=29.4 \mathrm{cal} \mathrm{g}^{-1} \quad(T=?)$
$L_{f}=123.0 \mathrm{~J} \mathrm{~g}^{-1}$

specific heat:

76.9/23.1 HMX/TNT

\begin{tabular}{|c|c|c|}
\hline temperature range, ${ }^{\circ} \mathrm{C}$ & $C p\left(\operatorname{cal} g^{-1} k^{-1}\right)$ & $C p\left(J g^{-1} k^{-1}\right)$ \\
\hline $\begin{array}{l}-79 \\
-80 \text { to } 80 \\
30 \text { to } 74 \\
90 \text { to } 150\end{array}$ & $\begin{array}{l}0.200 \\
0.240 \\
0.245 \\
0.323\end{array}$ & $\begin{array}{l}0.837 \\
1.004 \\
1.025 \\
1.351\end{array}$ \\
\hline
\end{tabular}




\section{EXPLOSIVES}

PENTOLITE; solid; an explosive mixture of trinitrotoluene (TNT) and pentaerythritol tetranitrate (PETN); 50/50 PETN/TNT; density $1.65 \mathrm{~g} \mathrm{~cm}^{3}$.

gross heat of combustion: $25^{\circ} \mathrm{C}$; calculated from the composition of the mixture.

$q_{v}($ gross $)=2774 \mathrm{cal} \mathrm{g}^{-1}$
$q_{v}$ (gross) $=11606 \mathrm{j}^{1}$

$[77,132]$

net heat of explosion: assume values refer to room temperature; reaction products unspecified.

$q_{v}($ net $)=1220 \mathrm{cal}^{-g^{-1}}$
$q_{v}($ net $)=5104 \mathrm{~J} \mathrm{~g}^{-1}$

[77]

explosion temperature after 5 seconds: decomposes, $220{ }^{\circ} \mathrm{C}$.

POLYVINYL NITRATE; solid; repeating unit: $\mathrm{C}_{2} \mathrm{H}_{3} \mathrm{NO}_{3}$.

gross heat of combustion: assume values refer to room temperature.

$q_{v}($ gross $)=2960 \mathrm{cal} \mathrm{g}_{1}^{-1}$
$q_{v}($ gross $)=12385 \mathrm{~J} \mathrm{~g}^{-1}$

net heat of explosion: assume values refer to room temperature; reaction products unspecified.

$q_{v}($ net $)=900 \mathrm{cal} \mathrm{g}^{-1}$
$q_{v}($ net $)=3766 \mathrm{Jg}^{1}$

explosion temperature after 5 seconds: $265^{\circ} \mathrm{C}$ 


\section{EXPLOSIVES}

TORPEX; solid; cyclotrimethylenetrinitramine (RDX), 42\%; trinitrotoluene (TNT), 40\%; aluminum, $18 \%$; density, cast $1.76-1.81 \mathrm{~g} \mathrm{~cm}^{-3}$.

gross heat of combustion: $25{ }^{\circ} \mathrm{C}$; calculated from composition of mixture.

$q_{v}($ gross $)=3727 \mathrm{cal} \mathrm{g}^{-1}$
$q_{v}($ gross $)=15594 \mathrm{~J} \mathrm{~g}^{1}$

$[77,132,134,138]$

net heat of explostion: assume values refer to room temperature; reaction products unspecified.

$q_{v}$ (net) $=1800 \mathrm{cal}^{-\mathrm{g}^{-1}}$
$q_{v}$ (net) $=7531 \mathrm{Jg}^{-1}$

specific heat: density, $1.82 \mathrm{~g} \mathrm{~cm}^{-3}$.

$\mathrm{Cp}\left(\mathrm{cal} \mathrm{g}^{-1} \mathrm{~K}^{-1}\right)=0.22\left(-5^{\circ} \mathrm{C}\right)$

$\mathrm{Cp}\left(\mathrm{J} \mathrm{g} \mathrm{g}^{-1} \mathrm{~K}^{-1}\right)=0.92\left(-5^{\circ} \mathrm{C}\right)$

$\mathrm{CP}\left(\mathrm{Cal} \mathrm{g}^{-1} \mathrm{~K}^{\mathrm{l}}\right)=0.24\left(15^{\circ} \mathrm{C}\right)$

$\mathrm{Cp}\left(\mathrm{J} \mathrm{g} \mathrm{g}^{-1} \mathrm{~K}^{-1}\right)=1.00\left(15^{\circ} \mathrm{C}\right)$

[77]

explosion temperature after 5 seconds: decomposes, $260^{\circ} \mathrm{C}$.

TRITONAL; solid; an explosive mixture of $80 \%$ trynitrotoluene (TMT) and $20 \%$ aluminum (Al): $80 / 20 \mathrm{TNT} / \mathrm{Al}$; density, cast, $1.72 \mathrm{~g} \mathrm{~cm}^{-3}$.

gross heat of combustion: $25{ }^{\circ} \mathrm{C}$; calculated from composition of mixture.

$q_{v}$ (gross) $=4352 \mathrm{cal} \mathrm{g}^{-1}$
$q_{v}$ (gross) $=18209 \mathrm{~J} \mathrm{~g}^{1}$

$[77,132,134]$

net heat of explosion: assume values refer to room temperature; reaction products unspecified.

$q_{v}$ (net) $=1770 \mathrm{cal} \mathrm{g}^{-1}$
$q_{v}$ (net) $=7406 \mathrm{~J} \mathrm{~g}^{-1}$

Specific heat: density, $1.74 \mathrm{~g} \mathrm{~cm}^{-3}$.

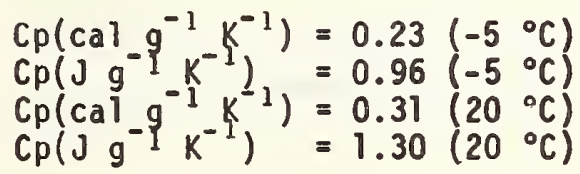

explosion temperature after 5 seconds: decomposes $470{ }^{\circ} \mathrm{C}$. 


\section{EXPLOSIVES}

VELTEX NO. 448; sol id; cyclotetramethylenetetranitramine (HMX), 70\%; nitrocellulose $(13.15 \%$ N) , $15 \%$; nitroglycerine, $10.7 \%$; 2-nitrodiphenylamine, $1.3 \%$; triacetin, $3.0 \%$; density, pressed, $1.72 \mathrm{~g} \mathrm{~cm}^{-3}$.

gross heat of combustion: assume values refer to room temperature.

$q_{v}($ gross $)=2359 \mathrm{cal}^{-g^{-1}}$
$q_{v}($ gross $)=9870 \mathrm{~J} \mathrm{~g}^{-1}$

net heat of explosion: assume values refer to room temperature; reaction products unspecified.

$q_{v}($ net $)=1226 \mathrm{cal}^{-1} \mathrm{~g}^{-1}$
$q_{v}($ net $)=5130 \mathrm{~J} \mathrm{~g}^{-1}$

FAT, WOOL; solid; (Lanolin), main constituents are cholesterol esters of higher molecular weight fatty acids.

gross heat of combustion: assume values refer to room temperature, and are corrected for moisture and ash content.

$q_{v}($ gross $)=9741 \pm 3.5 \mathrm{cal} \mathrm{g}^{-1}$
$q_{v}($ gross $)=40756 \pm 14.6 \mathrm{~J} \mathrm{~g}^{-1}$

FOODS

ALMONDS, Prunus amygdalus; solid; $4.4 \%$ moisture.

gross heat of combustion: assume values refer to room temperature.

$$
\begin{aligned}
& q_{v}(\text { gross })=3129 \mathrm{cal} \mathrm{g}^{-1} \\
& q_{v}(\text { gross })=13092 \mathrm{~J} \mathrm{~g}^{1}
\end{aligned}
$$

BEANS, NAVY, WHITE, DRY, Phaseolus vulgaris; solid; $11.2 \%$ moisture.

gross heat of combustion: assume values refer to room temperature.

$q_{v}($ gross $)=3900 \mathrm{cal} \mathrm{g}^{-1}$
$q_{v}($ gross $)=16320 \mathrm{~J} \mathrm{~g}^{1}$

BEETS, Beta vulgaris; solid; $82.6 \%$ moisture.

gross heat of combustion: assume values refer to room temperature.

$q_{v}($ gross $)=673 \mathrm{cal} \mathrm{g}^{-1}$
$q_{v}($ gross $)=2816 \mathrm{~J} \mathrm{~g}^{1}$ 
$\underline{\text { FOODS }}$

CABBAGE, Brassica oleracea var. capitata; solid; $94.6 \%$ moisture.

gross heat of combustion: assume values refer to room temperature.

$q_{v}($ gross $)=210 \mathrm{cal} \mathrm{g}^{-1}$
$q_{v}($ gross $)=880 \mathrm{~J} \mathrm{~g}^{-1}$

CITRUS FRUIT, RINDS AND SEEDS; solid; Uitimate analysis: carbon, $44.11 \%$; hydrogen, $5.22 \%$; oxygen, $38.34 \%$; nitrogen, $1.02 \%$; sulfur, $0.11 \%$; ash, $3.18 \%$ mo is ture, $8.02 \%$;

proximate analysis as received: moisture, $8.02 \%$; volatile matter, $71.46 \%$; fixed carbon, $17.34 \%$; ash, $3.18 \%$.

gross heat of combustion: assume values refer to room temperature.

as received:

$q_{v}$ (gross) $=949 \mathrm{cal} \mathrm{g}^{-1}$
$q_{v}($ gross $)=3970 \mathrm{~J} \mathrm{~g}^{1}$

air dried:

$q_{v}$ (gross) $=4098 \mathrm{cal} \mathrm{g}^{-1}$
$q_{v}$ (gross) $=17146 \mathrm{Jg}^{1}$

COCONUT, Cocos nucifera; solid; meat; $19.2 \%$ moisture.

gross heat of combustion: assume values refer to room temperature.

$q_{v}($ gross $)=2712 \mathrm{cal} \mathrm{g}^{-1}$
$q_{v}($ gross $)=11347 \mathrm{Jg}^{1}$

CORN (MAIZE), GREEN, Zea mays; solid; $76.0 \%$ moisture.

gross heat of combustion: assume values refer to room temperature.

$q_{k}($ gross $)=1112 \mathrm{cal}^{-g^{-1}}$
$q_{v}($ gross $)=4653 \mathrm{Jg}^{-1}$

COWPEAS, Vigna sinesis; solid; $10.0 \%$ moisture.

gross heat of combustion: assume values refer to room temperature.

$q_{v}($ gross $)=4000 \mathrm{cal} \mathrm{g}_{1}^{-1}$
$q_{v}($ gross $)=16736 \mathrm{Jg}^{2}$ 
EGG ALBUPIN; solid; a crystallizable protein; molecular weight about 33,800 ; from egg white.

gross heat of combustion: assume values refer to room temperature.

$q_{y}($ gross $)=5710 \mathrm{cal} \mathrm{g}^{-1}$
$q_{v}($ gross $)=23891 \cdot \mathrm{J} \mathrm{g}^{1}$

specific heat: temperature range: $0-25{ }^{\circ} \mathrm{C}$; anhydrous

$\begin{array}{ll}\operatorname{Cp}\left(\mathrm{cal}^{-} \mathrm{g}^{-1} \mathrm{~K}^{-1}\right) & =0.267 \\ \operatorname{Cp}\left(\mathrm{Jg}^{-1} \mathrm{~K}^{-1}\right) & =1.117\end{array}$

EGG YOLK; solid; yellow, semi-solid material of an egg; specific gravity, 0.95: high chloresterol content.

gross heat of combustion: assume values refer to room temperature.

$q_{v}$ (gross) $=5840 \mathrm{cal} \mathrm{g}^{-1}$

$q_{v}^{v}($ gross $)=24435 \mathrm{~J} \mathrm{~g}^{2}$

FAT, ANIMAL; solid; mixture of glycerides of fatty acids.

gross heat of combustion: assume values refer to room temperature.

$q_{v}($ gross $)=9500 \mathrm{cal} \mathrm{g}^{-1}$
$q_{v}($ gross $)=39750 \mathrm{~J} \mathrm{~g}^{2}$

whole animal:

$q_{v}($ gross $)=9499 \mathrm{cal} \mathrm{g}^{-1}$
$q_{v}($ gross $)=39744 \mathrm{~J} \mathrm{~g}_{1}$

FAT, BARLEY; solid; ether extracted.

gross heat of combustion: assume values refer to room temperature.

$\begin{aligned} q_{v}(\text { gross }) & =9070 \mathrm{cal} \mathrm{g}^{-1} \\ q_{v}(\text { gross }) & =37950 \mathrm{~J} \mathrm{~g}^{1}\end{aligned}$ 
FAT, BEEF; solid.

gross heat of combustion: assume values refer to room temperature.

$q_{v}($ gross $)=9500 \mathrm{cal} \mathrm{g}^{-1}$
$q_{v}($ gross $)=39750 \mathrm{~J} \mathrm{~g}^{1}$

fat obtained by ether extraction.

$q_{v}($ gross $)=9240 \mathrm{cal} \mathrm{g}^{-1}$
$q_{v}($ gross $)=38660 \mathrm{~J} \mathrm{~g}^{1}$

FAT, BUTTER; solid; processed from cream of cow's milk; composed mainly of glycerides (90\%) of oleic, palmitic and stearic acids; remainder (10\%) is made up of glycerides of butyric, capric, caprylic and caproic acids. Saponification Number 210-230.

gross heat of combustion: assume values refer to room temperature.

$q_{v}($ gross $)=9270 \mathrm{cal} \mathrm{g}^{-1}$
$q_{v}($ gross $)=38785 \mathrm{~J} \mathrm{~g}^{1}$

FAT, FRIED; solid; Ultimate analysis; carbon, $73.14 \%$; hydrogen, $11.54 \%$; oxygen, $14.28 \%$; nitrogen, $0.43 \%$; sulfur, $0.07 \%$; ash, $0.00 \%$; proximate analysis as received: moisture, $0.00 \%$; volatile matter, $97.64 \%$; fixed carbon, $2.36 \%$; ash, $0.00 \%$.

gross heat of combustion: assume values refer to room temperature.

$q_{v}($ gross $)=9154 \mathrm{cal} \mathrm{g}^{-1}$
$q_{v}($ gross $)=38300 \mathrm{~J} \mathrm{~g}^{1}$

FAT, LARD; solid; purified internal fat of the hog; fatty acid composition (typical): myristic $1.1 \%$; palmitic $30.4 \%$; stearic $17.9 \%$; oleic $41.2 \%$; linoleic $5.7 \%$. Iodine no. 46-66.

gross heat of combustion: assume values refer to room temperature.

$q_{v}($ gross $)=9590 \mathrm{cal} \mathrm{g}_{1}^{-1}$

$q_{v}($ gross $)=40120 \mathrm{~J} \mathrm{~g}^{1}$ 
FAT, MUTTON; solid.

gross heat of combustion: assune values refer to room temperature.

$q_{v}($ gross $)=9510 \mathrm{cal} \mathrm{g}^{-1}$
$q_{v}($ gross $)=39790 \mathrm{~J} \mathrm{~g}^{1}$

fat obtained by ether extraction

$q_{v}($ gross $)=9320 \mathrm{cal} \mathrm{g}_{1}^{-1}$
$q_{v}($ gross $)=39000 \mathrm{~J} \mathrm{~g}^{-1}$

FAT, PORK; solid.

gross heat of combustion: assume values reter to room temperature.

$q_{v}($ gross $)=9500 \mathrm{cal} \mathrm{g}^{-1}$
$q_{v}($ gross $)=39750 \mathrm{Jg}^{-1}$

[9]

fat obtained by ether extraction

$q_{v}($ gross $)=9130 \mathrm{cal} \mathrm{g}^{-1}$
$q_{v}($ gross $)=38200 \mathrm{~J} \mathrm{~g}^{-1}$

FAT, OAT, Avena sativa; solid; ether extract.

gross heat of combustion: assume values refer to room temperature.

$q_{v}($ gross $)=9070 \mathrm{cal} \mathrm{g}^{-1}$
$q_{v}($ gross $)=37950 \mathrm{~J} \mathrm{~g}^{1}$

FAT, RYE, Secale cereale; solid; ether extract.

gross heat of combustion: assume values refer to room temperature.

$q_{v}($ gross $)=9020 \mathrm{cal} \mathrm{g}^{-1}$
$q_{v}($ gross $)=37740 \mathrm{~J} \mathrm{~g}^{1}$ 
FAT, WHEAT, Triticum aestivum; solid; ether extract.

gross heat of combustion: assume values refer to room temperature.

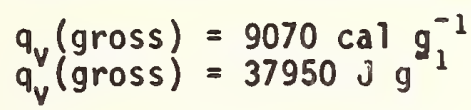

FLOUR, SOY BEAN, SOIa hispida.

gross heat of combustion: assume values refer to room temperature.

$4.17 \%$ moisture, $6.5 \%$ fat

$q_{v}($ gross $)=3716 \mathrm{cal} \mathrm{g}^{-1}$
$q_{v}($ gross $)=15548 \mathrm{~J} \mathrm{~g}^{1}$

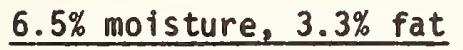

$q_{v}($ gross $)=3480 \mathrm{cal} \mathrm{g}^{-1}$
$q_{v}($ gross $)=14560 \mathrm{~J} \mathrm{~g}^{1}$ 
FLOUR, WHEAT; solid; the finely ground and bolted meal of wheat, triticum aestivum density $0.73 \mathrm{~g} \mathrm{~cm}^{-3}$.

net heat of explosion: nonisothermal in air; temperature $2250{ }^{\circ} \mathrm{C}$.

$q_{p}($ net $)=185 \mathrm{cal}^{-\mathrm{g}^{-1}}$
$q_{p}($ net $)=774 \mathrm{~J} \mathrm{~g}^{-1}$

specific heat:

$100-250{ }^{\circ} \mathrm{C}$

$\left.C p(c a) g^{-1} K^{-1}\right)=0.5$

$\operatorname{Cp}\left(\mathrm{J} \mathrm{g}^{-1} \mathrm{~K}^{-1}\right)=2.1$

[76]

gross heat of combustion: assume values refer to room temperature.

graham, whole grain; $10.5 \%$ mo is ture

$q_{v}($ gross $)=4004 \mathrm{cal} \mathrm{g}^{-1}$
$q_{v}($ gross $)=16750 \mathrm{~J} \mathrm{~g}^{1}$

[9]

standard patent; $10.3 \%$ moisture

$q_{v}($ gross $)=4010 \mathrm{cal} \mathrm{g}^{-1}$
$q_{v}($ gross $)=16780 \mathrm{~J} \mathrm{~g}^{1}$

[9]

semolina; $7.57 \%$ moisture

$q_{v}($ gross $)=4160 \mathrm{cal} \mathrm{g}^{-1}$
$q_{v}($ gross $)=17405 \mathrm{~J} \mathrm{~g}^{1}$

FOOD WASTES, YEGETABLE; solid; UItimate analysis: carbon, $49.06 \%$; hydrogen, $6.62 \%$; oxygen, $37.55 \%$; nitrogen, $1.68 \%$; sulfur, $0.20 \%$; ash, $4.8 \%$; proximate analysis as received: moisture, $78.29 \%$; volatile matter, $17.10 \%$; fixed carbon, $3.55 \%$; ash, $1.06 \%$.

gross heat of combustion: assume values refer to room temperature.

(as received)

$q_{v}($ gross $)=998 \mathrm{cal} \mathrm{g}^{-1}$
$q_{v}($ gross $)=4175 \mathrm{~J} \mathrm{~g}^{1}$

(dry basis)

$q_{v}($ gross $)=4598 \mathrm{cal} \mathrm{g}^{-1}$
$q_{v}($ gross $)=19236 \mathrm{~J} \mathrm{~g}^{1}$ 
HOMINY; from corn (maize), Zea mays; $10.96 \%$ moisture.

gross heat of combustion: assume values refer to room temperature.

$q_{v}($ gross $)=3986 \mathrm{cal} \mathrm{g}^{-1}$
$q_{v}($ gross $)=16677 \mathrm{~J} \mathrm{~g}^{-1}$

MEAL, CORN (MAIZE) Zea mays; $11.79 \%$ moisture.

gross heat of combustion: assume values refer to room temperature.

$q_{v}($ gross $)=3823 \mathrm{cal} \mathrm{g} \mathrm{g}^{-1}$
$q_{v}($ gross $)=15995 \mathrm{~J} \mathrm{~g}^{1}$

MEAT, COOKED SCRAPS; solid; U1timate analysis: carbon, $59.59 \%$; hydrogen, $9.47 \%$;

oxygen, 24.65\%; nitrogen, 1.02\%; sulfur, $0.19 \%$; ash, $5.08 \%$; proximate

analysis as received: moisture, $38.74 \%$; volatile matter, $56.34 \%$; fixed carbon, $1.81 \%$; ash, $3.11 \%$.

gross heat of combustion: assume values refer to room temperature.

as received:

$q_{v}($ gross $)=4238 \mathrm{cal} \mathrm{g}^{-1}$
$q_{v}($ gross $)=17731 \mathrm{Jg}^{2}$

dry basis:

$q_{v}($ gross $)=6917 \mathrm{cal} \mathrm{g}^{-1}$
$q_{v}($ gross $)=28942 \mathrm{~J} \mathrm{~g}^{2}$

OATS, Avena sativa; solid; rolled; $8.66 \%$ moisture.

gross heat of combustion: assume values refer to room temperature.

$q_{v}($ gross $)=4560 \mathrm{cal} \mathrm{g}^{-1}$
$q_{v}($ gross $)=19080 \mathrm{~J} \mathrm{~g}^{1}$

OIL, BARLEY; liquid; oil from barley, Hordeum vulgare; obtained by ether extraction.

gross heat of combustion: assume values refer to room temperature.

$q_{v}($ gross $)=9070 \mathrm{cal} \mathrm{g}^{-1}$
$q_{v}($ gross $)=37950 \mathrm{~J} \mathrm{~g}^{2}$ 
OIL, COCONUT; 1iquid; extracted from coconut meat, Cocos nucifera; mixture of glycerides of lauric ( $248 \%)$ and myristic $(217.5 \%)$ acids; remainder made up of glycerides of capyrlic, capric, palmitic, stearic, oleic and linoleic acids. Saponification Number 253-262.

gross heat of combustion: assume values refer to room temperature.

$q_{v}($ gross $)=9070 \mathrm{cal} \mathrm{g}^{-1}$
$q_{v}($ gross $)=37950 \mathrm{Jg}^{1}$

OIL, CORN (MAIZE); liquid; extracted from corn (maize), Zea mays; typical analysis: triglycerides $98.6 \%$; unsaponifiable $1.4 \%$; sitosteral $1 \%$; fatty acid composition: palmitic $2 \%$; stearic $3.5 \%$; oleic $46.3 \%$; linoleic $42.0 \%$. Iodine value 117-130.

gross heat of combustion: assume values refer to room temperature.

$q_{v}($ gross $)=9280 \mathrm{cal} \mathrm{g}^{-1}$
$q_{v}($ gross $)=38830 \mathrm{~J} \mathrm{~g}^{2}$

OIL. COTTONSEED, HYDROGENATED; liquid; yellow oil from various Gossipyium species; chief constituents are glycerides of palmitic $(221 \%)$; oleic ( $23 \%$ ); stearic $(\sim 2 \%)$; and linoleic ( $44 \%$ ) acids; density $0.91-0.92 \mathrm{~g} \mathrm{~cm}^{-3}$; before measurement oil was heated at $100{ }^{\circ} \mathrm{C}$ for 3 to 6 hours at $2-3 \mathrm{~mm}$ of $\mathrm{Hg}$ to remove moisture; iodine no. was 6.5 .

specific heat:

$80^{\circ}-270^{\circ} \mathrm{C}$

$\operatorname{Cp}\left(\operatorname{cal}^{-1} \mathrm{q}^{-1} \mathrm{~K}^{-1}\right)=0.5363-4.7376 \times 10^{-4} \mathrm{~T}+1.2398 \times 10^{-6} \mathrm{~T}^{2}$
$\operatorname{Cp}\left(\mathrm{J} \mathrm{g}^{-1} \mathrm{~K}^{-1}\right)=2.2439-1.9822 \times 10^{-3} \mathrm{~T}+5.1875 \times 10^{-6} \mathrm{~T}^{2}$

OIL, OAT; liquid; obtained from oats; Avena saliva; obtained by ether extraction. gross heat of combustion: assume values refer to room temperature.

$q_{v}($ gross $)=8930 \mathrm{cal} \mathrm{g}^{-1}$
$q_{v}($ gross $)=37365 \mathrm{~J} \mathrm{~g}^{1}$ 
OIL, OLIVE; liquid; mixture of glycerides of several fatty acids; chiefly glycerides of oleic acid, with lesser amounts of palmitic, stearic, and linoleic acids. Saponification Number 185-196.

gross heat of combustion: assume values refer to room temperature.

$q_{v}($ gross $)=9470 \mathrm{cal} \mathrm{g}^{-1}$
$q_{v}($ gross $)=39620 \mathrm{~J} \mathrm{~g}^{1}$

vapor pressure: $245^{\circ}-310^{\circ} \mathrm{C}$

$\log _{10} P($ Torr $)=17.92-9430 \mathrm{~T}^{-1}$
$\log _{10} \mathrm{P}(\mathrm{Pa})=20.05-9430 \mathrm{~T}^{-1}$

OIL. RYE; liquid; oil extracted from rye grain, Secale cereale.

gross heat of combustion: assume values refer to room temperature.

$q_{v}($ gross $)=9320 \mathrm{cal} \mathrm{g}^{-1}$
$q_{v}($ gross $)=38990 \mathrm{~J} \mathrm{~g}^{2}$

obtained by ether extraction:

$q_{v}($ gross $)=9200 \mathrm{cal} \mathrm{g}^{-1}$
$q_{v}($ gross $)=38495 \mathrm{~J} \mathrm{~g}^{1}$

OIL, SOYBEAN; liquid; the bean of Soia hispida (Leguminosae); average composition: proteins $40 \%$; oils $18 \%$; phosphatides $2 \%$; remainder urease, raffinose, stachyose, saponins, phytosterins, and isoflavone; density is $0.925 \mathrm{at}$. $15^{\circ} \mathrm{C}$; saponification no. 189-193.5; iodine no. 122-134.

vapor pressure: $250^{\circ}-285^{\circ} \mathrm{C}$

$\begin{array}{ll}\log _{10} P(T o r r) & =18.30-9650 \mathrm{~T}^{-1} \\ \log _{10} P(\mathrm{~Pa}) & =20.42-9650 \mathrm{~T}^{-1}\end{array}$

specific heat: sample was heated at $100{ }^{\circ} \mathrm{C}$ for 3 to 6 hours under a pressure of $2-3 \mathrm{~mm}$ of $\mathrm{Hg}$ to remove moisture.

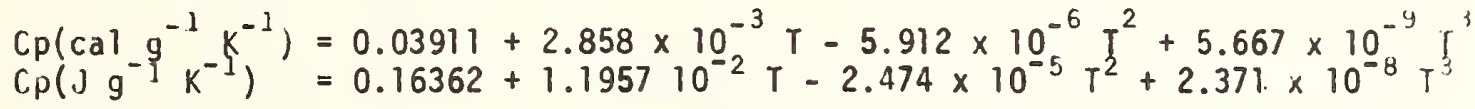


OIL, WHEAT; liquid; from wheat grain, Triticum acestivum.

gross heat of combustion: assume values refer to room temperature.

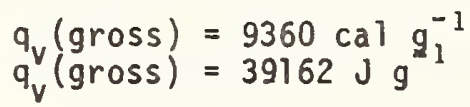

oil obtained by ether extraction:

$q_{v}($ gross $)=9070 \mathrm{cal} \mathrm{g}^{-1}$
$q_{v}($ gross $)=37950 \mathrm{~J} \mathrm{~g}^{2}$

PECANS, Carya 111 inoensis; solid, $4.30 \%$ moisture.

gross heat of combustion: assume values refer to room temperature.

$q_{v}($ gross $)=3551 \mathrm{cal} \mathrm{g}^{-1}$
$q_{v}($ gross $)=14857 \mathrm{~J} \mathrm{~g}^{-1}$

PEANUTS, Arachis hypogaea; solid; $4.88 \%$ moisture.

gross heat of combustion: assume values refer to room temperature.

$q_{v}($ gross $)=3040 \mathrm{cal} \mathrm{g}^{-1}$
$q_{v}($ gross $)=12720 \mathrm{~J} \mathrm{~g}^{1}$

POTATO, WHITE; solid; approximate analysis: moisture, $79.5 \%$; carbohydrates $16.7 \%$; protein, $2 \%$; fiber, $0.4 \%$; ash, $0.8 \%$; and fat, $0.1 \%$.

gross heat of combustion: assume values refer to room temperature.

$q_{v}($ gross $)=848 \mathrm{cal} \mathrm{g}^{-1}$
$q_{v}($ gross $)=3548 \mathrm{~J} \mathrm{~g}^{1}$

specific heat: at $30^{\circ} \mathrm{C}$, with a moisture content $(W)$ range of 0.23 to 0.83 wt. fraction.

$(W>0.50)$

$C p\left(c a 1 g^{-1} K^{-1}\right)=0.216+0.780 W$

$C p\left(J g^{-1} K^{-1}\right)=0.904+3.264 W$

$(0.50, W>0.20)$

$\left.C p(c a) g^{-1} K^{-1}\right)=0.393+0.437 W$

$C p\left(J g^{-1} K^{-1}\right)=1.644+1.828 W$ 
FOODS

RICE, Oryza sativa; solid; white; polished; $12.9 \%$ moisture.

gross heat of combustion: assume values refer to room temperature.

$q_{v}($ gross $)=3854 \mathrm{cal} \mathrm{g}^{-1}$
$q_{V}($ gross $)=16125 \mathrm{~J} \mathrm{~g}^{2}$

WALNUTS, ENGLISH, Juglans regia; solid; $3.97 \%$ moisture.

gross heat of combustion: assume values refer to room temperature.

$q_{v}($ gross $)=3318 \mathrm{cal} \mathrm{g_{1 } ^ { - 1 }}$

$q_{v}($ gross $)=13882 \mathrm{~J} \mathrm{~g}^{2}$

WHEAT, Triticum aestivum; $8.5 \%$ moisture.

gross heat of combustion: assume values refer to room temperature.

$q_{v}($ gross $)=4090 \mathrm{cal} \mathrm{g}^{-1}$
$q_{v}($ gross $)=17115 \mathrm{~J} \mathrm{~g}^{2}$ 
FUEL, AVIATION; 1 iquid; petroleum fraction with B.P. range $175^{\circ}-350{ }^{\circ} \mathrm{C}$ (kerosine).

Military jet fuels labeled JP-3, JP-4,JP-5, etc. Commercial jet fuels

labeled as ASTM types $A, A-1$, or $B$.

net heat of combustion: $A$ is the aniline point, in degrees Fahrenheit, identifying the minimum equilibrium solution temperature for equal volumes of aniline and sample. G is the API gravity defined as: API gravity (deg) $=141.5 /$ (sp. gr. 60/60 ${ }^{\circ} \mathrm{F}$ ) -131.5. Tables are provided in ASTM Specification 01405 to calculate $q_{p}$ (net) if sulfur impurities are known.

Aviation gasoline, Grades 100-130 and 115-145:

$q_{p}($ net $)=(10027+0.0463(A \times G)) \mathrm{Cal}^{-g^{-1}}$
$q_{p}($ net $)=(41954+0.1938(A \times G)) \mathrm{Jg}^{-1}$

Jet fuels:

$\underline{J P-3}$

$q_{p}($ net $)=(9973+0.0587(A \times G)) \mathrm{Cal} \mathrm{g}^{-1}$
$q_{p}($ net $)=(41728+0.2456(A \times G)) \mathrm{J} \mathrm{g}^{1}$

$\mathrm{JP}-4$

$q_{p}($ net $)=(9994+0.0587(A \times G)) \mathrm{cal} \mathrm{g}^{-1}$

$q_{p}^{p}($ net $)=(41815 \times 0.2456(A \times G)) \mathrm{Jg}^{g}$

$\underline{J P-5}$

$q_{p}($ net $)=(9959+0.0587(A \times G)) \mathrm{Cal} \mathrm{g}^{-1}$
$q_{p}($ net $)=(41668+0.2456(A \times G)) \mathrm{J} \mathrm{g}^{2}$

Kerosine, Jet A or A-1 (see ASTM Speciation D 1655):

$q_{p}($ net $)=(9962+0.06072(A \times G)) \mathrm{cal} \mathrm{g}^{-1}$
$q_{p}($ net $)=(41680+0.25407(A \times G)) \mathrm{J} \mathrm{g}^{1}$

FUEL, GASOLINE; liquid; a mixture of volatile hydrocarbons with boiling points ranging from $140^{\circ}$ to $390^{\circ} \mathrm{F}$ (branched-chain paraffins, cycloparaffins, and aromatics).

auto-ignition temperature:

octane rating

65

73

87

\section{temperature}

${ }^{\circ} \mathrm{C} \quad{ }^{\circ} \mathrm{F}$

$248 \quad 478$

$258 \quad 496$

$412 \quad 774$ 
FUEL, KEROSINE; liquid; mixture of hydrocarbons with boiling points ranging from $180^{\circ}$ to $300^{\circ} \mathrm{C}$; ultimate composition: carbon, $85.8 \%$; hydrogen, $14.2 \%$; sulfur, $58 \mathrm{ppm}$; total paraffins, $30.9 \%$; total naphthenes, $64.3 \%$; tota 7 aromatics, $4.8 \%$; density, $0.8019 ; \mathrm{g} \mathrm{cm}^{-3}$ at $24^{\circ} \mathrm{C}$; API gravity, 43.5 .

gross heat of combustion: assume values refer to room temperature.

$q_{v}($ gross $)=11087 \mathrm{cal}^{-g^{-1}}$
$q_{v}($ gross $)=46390 \mathrm{~J} \mathrm{~g}^{-1}$

GAS, NATURAL; gas; composition in mole percent; (sample A), $N_{2}=15.6 \%, 11 \mathrm{e}=0.84 \%$, $\mathrm{CO}_{2}=0.03 \%, \mathrm{CH}_{4}=72.2 \%, \mathrm{C}_{2} \mathrm{H}_{6}=6.5 \%$, propane $=3.8 \%$, n-butarle $=0.66 \%$, isobutane $=0.28 \%$, pentanes $=0.02 \%$, hexanes $=0.01 \%$, and heptanes $=0.01 \%$. (sample B), $\mathrm{N}_{2}=2.8 \%, \mathrm{He}=0.23 \%, \mathrm{CO}_{2}=0.19 \%, \mathrm{CH}_{4}=86.2 \%, \mathrm{C}_{2} \mathrm{H}_{6}=6.2 \%$, propane $=2.7 \%$, $n$-butane $=0.63 \%$, isobutane $=0.52 \%$, pentanes $=0.32 \%$, hexanes $=$ $0.22 \%$, and heptanes $=0.07 \%$. (sample C), $\mathrm{N}_{2}=0.24 \%, \mathrm{He}=0.00 \%, \mathrm{CO}_{2}=0.87 \%$, $\mathrm{CH}_{4}=94.7 \%, \mathrm{C}_{2} \mathrm{H}_{6}=3.2 \%$, propane $=0.67 \%$, n-butane $=0.12 \%$, isobutane $=0.06 \%$, pentanes $=0.06 \%$, hexanes $=0.06 \%$, and heptanes $=0.04 \%$. (sample $\mathrm{D}), \mathrm{N}_{2}=7.1 \%$ $\mathrm{He}=0.32 \%, \mathrm{CO}_{2}=0.49 \%, \mathrm{CH}_{4}=84.3 \%, \mathrm{C}_{2} \mathrm{H}_{6}=5.2 \%$, propane $=1.9 \%$, n-butane $=$ $0.31 \%$, isobutane $=0.28 \%$, pentanes $=0.04 \%$, hexanes $=0.04 \%$, and heptanes $=$ $0.03 \%$.

gross heat of combustion: at $60^{\circ} \mathrm{F}\left(15.56^{\circ} \mathrm{C}\right)$ and $1 \mathrm{~atm}(101325 \mathrm{~Pa})$; where SCF is standard cubic foot.

(sample A)

$q_{v}($ gross $)=958 \mathrm{Btu}(\mathrm{SCF})^{-1}$

$q_{v}($ gross $)=35.7 \times 10^{6} \mathrm{~J} \mathrm{~m}^{-3}$

(sample B)

$q_{v}$ (gross) $=1101 \mathrm{Btu}(\text { SCF })^{-1}$

$q_{v}($ gross $)=41.0 \times 10^{6} \mathrm{~J} \mathrm{~m}^{-3}$

(sample C)

$q_{v}$ (gross) $=1028 \mathrm{Btu}(\text { SCF })^{-1}$

$q_{v}^{v}($ gross $)=38.3 \times 10^{6} \mathrm{~J} \mathrm{~m}^{-3}$

(sample 0 )

$q_{v}$ (gross) $=999 \mathrm{Btu}(S C F)^{-1}$
$q_{v}($ gross $)=37.2 \times 10^{6} \mathrm{~J} \mathrm{~m}^{-3}$

compressibility factor, $Z=P V / R T$ : at $60{ }^{\circ} \mathrm{C}\left(15.56{ }^{\circ} \mathrm{C}\right)$ and 1 atm $(101325 \mathrm{~Pa})$.

$Z($ sample $A)=0.9978$

$Z($ sample B $)=0.9974$

$Z($ sample $C)=0.9978$

$Z($ sample D) $=0.9979$ 
GLASS, PYREX; solid (Corning 7740); $\mathrm{SiO}_{2} 80.66 \%, \mathrm{~B}_{2} \mathrm{O}_{3} 12.93 \%, \mathrm{Na}_{2} \mathrm{O} 3.80 \%, \mathrm{Al}_{2} \mathrm{O}_{3} 2.14 \%$, and $\mathrm{K}_{2} \mathrm{O} 0.46 \%$.

heat content:

$.373-850 k$

$q_{T}-q_{273.8}\left(\mathrm{cal} \mathrm{g} \mathrm{g}^{-1}\right)=-94.88+0.2325 \mathrm{~T}+4.39 \times 10^{-5} \mathrm{~T}^{2}+8.083 \times 10^{3} \mathrm{~T}^{-1}$

${ }^{q_{T}} \mathrm{~T}_{273.8}\left(\mathrm{~J} \mathrm{~g}^{-1}\right)=-396.98+0.9728 \mathrm{~T}+1.837 \times 10^{-4} \mathrm{~T}^{2}+3.382 \times 10^{4} \mathrm{~T}^{-1}$

[18]

\section{specific heat:}

$173-293 k$

$\operatorname{Cp}\left(\operatorname{cat} g^{-1} K^{-1}\right)=-0.245+2.63 \times 10^{-3} \mathrm{~T}-3.80 \times 10^{-6} \mathrm{~T}^{2}$

[10]

$373-850 k$

$C p\left(\right.$ cal $\left.^{-1} \mathrm{~K}^{-1}\right)=0.2325+8.78 \times 10^{-5} \mathrm{~T}-8.083 \times 10^{3} \mathrm{~T}^{-2}$

$\mathrm{Cp}\left(\mathrm{J} \mathrm{g} \mathrm{g}^{-1} \mathrm{~K}^{-1}\right)=0.9728+3.674 \times 10^{-4} \mathrm{~T}-3.382 \times 10^{4} \mathrm{~T}^{-2}$

[18]

GLASS, PYREX: 1iquid; (Corning 7740); $\mathrm{SiO}_{2} 80.66 \%, \mathrm{~B}_{2} \mathrm{O}_{3} 12.93 \%, \mathrm{Na}_{2} \mathrm{O} 3.80 \%, \mathrm{Al}_{2} \mathrm{O}_{3} 2.14 \%$, $\mathrm{K}_{2} \mathrm{O}, 0.46 \%$.

specific heat: over temperature range 850 to $1200 \mathrm{~K}$ the specific heat appears to be independent of temperature.

$\left.C p(c a) g^{-1} K^{-1}\right)=0.3471$

$C p\left(\mathrm{~J} \mathrm{~g}^{-1} \mathrm{~K}^{-1}\right)=1.4523$

heat content:

$850-1200 k$

$q_{T}-q_{273.8}\left(\right.$ cal g $\left.^{-1}\right)=-152.28=0.3471 \mathrm{~T}$

$\mathrm{q}_{\mathrm{T}}-\mathrm{q}_{273.8}\left(\mathrm{~J} \mathrm{~g}^{-1}\right)=-637.14+1.4523 \mathrm{~T}$

[18] 
GLASS, SOOA-LIME; solid; (Lillie 1); $\mathrm{SiO}_{2} 73.59 \%, \mathrm{Na}_{2} \mathrm{O} 16.65 \%, \mathrm{Ca} 09.76 \%$,

specific heat:

$273-850 k$

$\left.C p(c a) g^{-1} K^{-1}\right)=0.1347+1.932 \times 10^{-4} T+2.423 \times 10^{3} T^{-2}$

$C p\left(\mathrm{~J} \mathrm{~g}^{-1} \mathrm{~K}^{-1}\right)=0.5636+8.083 \times 10^{-4} \mathrm{~T}+1.0138 \times 10^{4} \mathrm{~T}^{-2}$

heat content:

$273-850 k$

$q_{T}-q_{273.8}\left(\mathrm{cal} \mathrm{g} \mathrm{g}^{-1}\right)=-36.37+0.1347 \mathrm{~T}+9.66 \times 10^{-5} \mathrm{~T}^{2}-2.423 \times 10^{3} \mathrm{~T}^{-1}$

$q_{T}-q_{273.8}\left(\mathrm{~J} \mathrm{~g}^{-1}\right)=-152.17+0.5636 \mathrm{~T}+4.042 \times 10^{-4} \mathrm{~T}^{2}-1.0138 \times 10^{4} \mathrm{~T}^{-1}$

GLASS, SODA-LIME; liquid; (Lillie l); $\mathrm{SiO}_{2} 73.59 \%, \mathrm{Na}_{2} \mathrm{O} 1.6 .65 \%, \mathrm{CaO} 9.76 \%$.

specific heat: over the temperature range 850 to $1200 \mathrm{~K}$, the specific

heat appears to be independent of temperature.

$C_{p}\left(c_{1} g^{-1} K^{-1}\right)=0.3570$

$C_{p}\left(\mathrm{~J} \mathrm{~g}^{-1} \mathrm{~K}^{-1}\right)=1.4937$

heat content:

850 to $1200 \mathrm{~K}$

$q_{T}-q_{273.8}\left(\operatorname{cal~} g^{-1}\right)=-158.81+0.3570 \mathrm{~T}$

$q_{T}-q_{273.8}\left(\mathrm{~J} \mathrm{~g}^{-1}\right)=-664.46+1.4937 \mathrm{~T}$

[18]

GLASS, VYCOR; solid; $\mathrm{SiO}_{2} 96.3 \%, \mathrm{~B}_{2} \mathrm{O}_{3} 2.9 \%, \mathrm{Al}_{2} \mathrm{O}_{3} 0.4 \%$, and other metal oxides $0.4 \%$.

specific heat: $0-306{ }^{\circ} \mathrm{C}$, mean values

$C p\left(\operatorname{cal} g^{-1} K^{-1}\right)=0.12393+1.4333 \times 10^{-4} \mathrm{~T}$ 
GRAPHITE, ARTIFICIAL; solid; National Carbon Co. spectroscopic grade.

gross heat of combustion: at $25{ }^{\circ} \mathrm{C}$, average of 2 samples.

$q_{v}($ gross $)=7035.8 \pm 2.4 \mathrm{cal} \mathrm{g}^{-1}$
$q_{v}($ gross $)=32785 \pm 10 \mathrm{Jg}^{-1}$

[142]

GRAPHITE, BUCKINGHAM; solid; $0.25 \%$ ash.

gross heat of combustion: at $25{ }^{\circ} \mathrm{C}$, corrected for ash content.

$q_{v}($ gross $)=7836.8 \pm 2.0 \mathrm{~J}^{-1} \mathrm{~g}^{-1}$
$q_{v}($ gross $)=32789 \pm 8 \mathrm{~g}^{-1}$

[142]

GRAPHITE, CEYLON; solid; $2.2 \%$ ash.

gross heat of combustion: at $25{ }^{\circ} \mathrm{C}$, corrected for ash content.

$q_{v}($ gross $)=7825.0 \pm 0.5 \mathrm{cal} \mathrm{g}^{-1}$
$q_{v}($ gross $)=32740 \pm 2 \mathrm{jg}^{-1}$

[142]

GRAPHITE, PYROIYIIC; sulid; The total gas content is less than 1 ppm; (sample 1) annealed at $2200{ }^{\circ} \mathrm{C}$, having a density of $2.204 \mathrm{~g} \mathrm{ml}^{-1}$; (sample 2) annealed at $3000^{\circ} \mathrm{C}$, having a density of $2.265 \mathrm{~g} \mathrm{ml}^{-1}$.

gross heat of combustion: at $298^{\circ} \mathrm{K}$

(sample 1)

$q_{v}($ gross $)=7844.9 \pm 1.7 \mathrm{cal} \mathrm{g}^{-1}$
$q_{v}($ gross $)=32823.1 \pm 7.1 \mathrm{~J} \mathrm{~g}^{1}$

(sample 2)

$q_{v}($ gross $)=7823.9 \pm 3.0 \mathrm{cal} \mathrm{g}^{-1}$
$q_{v}($ gross $)=32735.2 \pm 12.6 \mathrm{Jg}^{-1}$

[64]

GRAPHITE, TICONUEROCA; SOlid; $0.33 \%$ ash.

gross heat of combustion: at $25^{\circ} \mathrm{C}$, corrected for ash content.

$q_{v}($ gross $)=7836.0 \pm 1.0 \mathrm{cal} \mathrm{g}^{-1}$
$q_{v}($ gross $)=32786 \pm 4 \mathrm{j}^{-\frac{1}{1}}$ 
GREASE, APIEZON N; solid; low vapor-pressure residues of paraffin oll distiliation products.

specific heat: 1 to $20 \mathrm{~K}$.

$C_{p}=\Sigma A_{n} T^{n}$, where $C_{p}$ is in $m J g^{-1} K^{-1}$ and $T$ is in $K_{\text {; }}$ values are valid to $\pm 1 \%$.

$80-200 k$

$$
\begin{aligned}
C p\left(\text { cal g } g^{-1} K^{-1}\right)= & -1.4486 \times 10^{-2}+2.3281 \times 10^{-3} \mathrm{~T} \\
& -8.0195 \times 10^{-6} \mathrm{~T}^{2}+1.8998 \times 10^{-8} \mathrm{~T}^{3} \\
C_{p}\left(\mathrm{Jg}^{-1} \mathrm{~K}^{-1}\right)= & -6.0609 \times 10^{-2}+9.7408 \times 10^{-3} \mathrm{~T} \\
& -3.3554 \times 10^{-5} \mathrm{~T}^{2}+7.2488 \times 10^{-8} \mathrm{~T}^{3}
\end{aligned}
$$

In the temperature range $200-324 K, C p$ is anomaious; values are given below:

$\begin{array}{lcc}T(K) & C p\left(c a l g^{-1} \mathrm{deg}^{-1}\right) & C p\left(J \mathrm{~g}^{-1} \mathrm{deg}^{-1}\right) \\ 200 & 0.2813 & 1.177 \\ 210 & 0.301 & 1.26 \\ 220 & 0.344 & 1.44 \\ 230 & 0.392 & 1.64 \\ 240 & 0.421 & 1.76 \\ 250 & 0.445 & 1.86 \\ 260 & 0.468 & 1.96 \\ 270 & 0.543 & 2.27 \\ 280 & 0.679 & 2.84 \\ 290 & 0.853 & 3.57 \\ 296 & 0.899 & 3.76 \\ 300 & 0.858 & 3.59 \\ 310 & 0.626 & 2.62 \\ 320 & 0.554 & 2.32 \\ 324 & 0.538 & 2.25\end{array}$

glass temperature: $236.5 \pm 0.5 \mathrm{~K}$ or $-36.7 \pm 0.5{ }^{\circ} \mathrm{C}$ 
GREASE, APIEZON P; solid; low vapor-pressure residues of paraffin oil distillation products; average molecular weight $410 \pm 70$.

heat of vaporization: temperature range $40^{\circ}$ to $160^{\circ} \mathrm{C}$.

$L_{v}=84.70 \mathrm{cal}^{-1}$
$L_{v}=354.4 \mathrm{Jg}^{-1}$

vapor pressure: temperature range $40^{\circ}$ to $160^{\circ} \mathrm{C}$.

$\log _{10} P($ Torr $)=13.91-7590 \mathrm{~T}^{-1}$

$\log _{10} \mathrm{P}(\mathrm{Pa})=16.03-7590 \mathrm{~T}^{-1}$

[59]

GREASE, APIEZON R; solid; low vapor-pressure residues of paraffin oil distillation products; average molecular weight $660 \pm 100$.

heat of vaporization: temperature range $40^{\circ}$ to $160^{\circ} \mathrm{C}$.

$L_{v}=48.88 \mathrm{cal}^{-1}$
$L_{v}=204.5 \mathrm{~J} \mathrm{~g}^{-1}$

vapor pressure: temperature range $40^{\circ}$ to $160^{\circ} \mathrm{C}$.

$\log _{10} P($ Torr $)=12.54-7050 \mathrm{~T}^{-1}$

$\log _{10} \mathrm{P}(\mathrm{Pa})=14.66-7050 \mathrm{~T}^{-1}$

[59]

GREASE, APIEZON S; solid; low vapor-pressure residues of paraffin oil distillation products; average molecular weight $460 \pm 60$.

heat of vaporization: temperature range $40^{\circ}$ to $160{ }^{\circ} \mathrm{C}$.

$L_{v}=70.08 \mathrm{cal} \mathrm{g}^{-1}$
$L_{v}=293.2 \mathrm{~J} \mathrm{~g}^{-1}$

vapor pressure: temperature range $40^{\circ}$ to $160^{\circ} \mathrm{C}$.

$\log _{10} P($ Torr $)=12.52-7045 \mathrm{~T}^{-1}$

$\log _{10} \mathrm{P}(\mathrm{Pa})=14.64-7045 \mathrm{~T}^{-1}$

[59] 
GREASE, APIEZON T; solid; low vapor-pressure residues of paraffin oil distillation products; also contains an aluminum soap [1].

specific heat: specific heat is anomajous between 205 and $320 \mathrm{~K}$.

\begin{tabular}{cll} 
temperature $(K)$ & $c p\left(\operatorname{cal}^{-1} \mathrm{~K}^{-1}\right)$ & \multicolumn{1}{c}{$\mathrm{Cp}\left(\mathrm{J} \mathrm{g}^{-1} \mathrm{~K}^{-1}\right)$} \\
5 & 0.001035 & 0.0004330 \\
50 & 0.07746 & 0.3241 \\
100 & 0.7518 & 0.6351 \\
150 & 0.2149 & 0.8997 \\
200 & 0.2811 & 1.1761 \\
220 & 0.362 & 1.515 \\
240 & 0.447 & 1.870 \\
260 & 0.498 & 2.084 \\
280 & 0.526 & 2.201 \\
300 & 0.517 & 2.163 \\
320 & 0.5022 & 2.101 \\
340 & 0.5180 & 2.167 \\
350 & 0.5259 & 2.200
\end{tabular}

glass temperature: $209.5 \pm 1.0 \mathrm{~K}$

INSULIN, BOVINE ZINC; solid; the anhydrous material from beef pancreas made up of 16 amino acids arranged in two chains connected by sulfur bridges,

$\left(\mathrm{C}_{508} \mathrm{H}_{752} \mathrm{O}_{150} \mathrm{~N}_{130} \mathrm{~S}_{12} \mathrm{Zn}\right)$. The ash content $=0.95 \%$; zinc present (as $\left.\mathrm{ZnO}\right)=0.67 \%$.

specific heat:

$\begin{array}{ccc}\text { temperature }(K) & \left.C p(c a) g^{-1} K^{-1}\right) & C p\left(\mathrm{~J} \mathrm{~g}^{-1} \mathrm{~K}^{-1}\right) \\ 10 & 0.00475 & 0.01987 \\ 100 & 0.1192 & 0.4987 \\ 200 & 0.2089 & 0.8740 \\ 250 & 0.2541 & 1.0632 \\ 298.15 & 0.2996 & 1.2535 \\ 300 & 0.3014 & 1.2611\end{array}$

[128] 
IRON, CAST; liquid; composition of cast iron: base iron ( $F e)$, the compound cellentite $\left(\mathrm{Fe}_{3} \mathrm{C}\right)$, and alloying elements of graphite (C), and silicon ( $\mathrm{Si}$ ), and lesser elements such as $\mathrm{Mn}, \mathrm{P}$, and S. A typical composition for ductile iron is $C=3.2-4.2 \%, S i=1.1-3.5 \%, M n=0.3-0.8 \%, P=0.08 \%, S=0.02 \%$, and remainder $\mathrm{Fe}$.

heat content: The temperature range is $2600^{\circ}$ to $2900^{\circ} \mathrm{F}(\sim 1400-1600$ " $\mathrm{C}$ ); i is in $\bar{K}$; the equation applies to all cast iron and is the sum of individual heat content equations where the lesser elements are ignored. $X$ is weight fraction of substance.

$$
\begin{aligned}
& q_{T}-q_{273}\left(\mathrm{cal} \mathrm{g}{ }^{-1}\right)=(0.1758 \mathrm{~T}-1.08) x_{\mathrm{Fe}}+(0.1758 \mathrm{~T}-2.98) x_{\mathrm{Fe}_{3} \mathrm{C}} \\
& +(0.5002 T+830.2) x_{C}+(0.2168 T+549.7) x_{S i}
\end{aligned}
$$

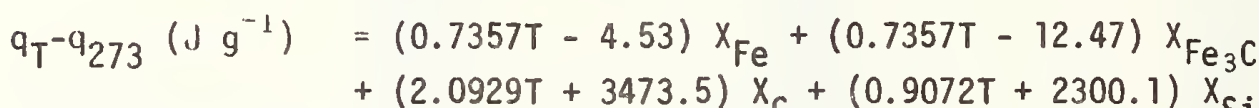

[63]

LEATHER; solid; an animal skin or hide which has been permanently combined with a tanning agent; the tanning agent produces a transformation in the protein composition of the skin rendering it resistant to putrefactive bacteria, increasing its strength and abrasion resistance.

specific heat: $25^{\circ} \mathrm{C}$

moisture content, \%

$$
C p\left(c a l g^{-1} K^{-1}\right) \quad C p\left(J g^{-1} K^{-1}\right)
$$

vegetable-tanned

$\begin{array}{rrr}0 & 0.3334 & 1.3949 \\ 1.15 & 0.3202 & 1.3397 \\ 1.32 & 0.3274 & 1.3698 \\ 2.59 & 0.3280 & 1.3724 \\ 3.85 & 0.3844 & 1.6083 \\ 6.20 & 0.3730 & 1.5606 \\ 7.83 & 0.3835 & 1.6046 \\ 9.31 & 0.3844 & 1.6083 \\ 10.81 & 0.4129 & 1.7276\end{array}$

chrome-tanned

$\begin{array}{rrr}0 & 0.3045 & 1.2740 \\ 1.98 & 0.2918 & 1.2209 \\ 3.69 & 0.3631 & 1.5192 \\ 7.59 & 0.3576 & 1.4962 \\ 9.39 & 0.3737 & 1.5636 \\ 11.07 & 0.3893 & 1.6288 \\ 13.46 & 0.3841 & 1.6071 \\ 14.96 & 0.4098 & 1.7146\end{array}$

chrome-retanned

$\begin{array}{rll}0 & 0.3404 & 1.4242 \\ 1.52 & 0.3287 & 1.3753 \\ 2.12 & 0.3266 & 1.3665 \\ 4.23 & 0.3448 & 1.4426 \\ 7.23 & 0.3458 & 1.4468 \\ 9.23 & 0.3474 & 1.4535 \\ 11.29 & 0.3606 & 1.5088\end{array}$


LIGNIN, LOBLOLLY PINE (Pinus Taeda L) WOOD; solid; major (29.4\% 1 ignin [81]) noncarbohydrate constituent of wood; functions as a natural piastic binder for cellulose fibers.

specific heat: 333 to $413 \mathrm{~K}$; samples were oven-dried at $100{ }^{\circ} \mathrm{C}$ for 12 hours before measurements were made.

$$
\begin{aligned}
& C p\left(\operatorname{cal} g^{-1} K^{-1}\right)=0.06133+7.7 \times 10^{-4} T \\
& C p\left(J g K^{-1}\right)=0.25660+3.22 \times 10^{-3} T
\end{aligned}
$$

LIGNIN, HARDWOOD: solid; composition: carbon, $60 \%$; hydrogen, $6 \%$; oxygen, $34 \%$; empirical formula: $\mathrm{C}_{10} \mathrm{H}_{12} \mathrm{O}_{4} .2$. No general agreement prevails about the structure of lignin. Studies show that a dioxyphenylpropyl grouping is an important part of the polymer.

gross heat of combustion: assume values refer to room temperature.

$$
\begin{aligned}
& q_{v}(\text { gross })=5900 \mathrm{cal} \mathrm{g}^{-1} \\
& q_{v}(\text { gross })=24685 \mathrm{~J} \mathrm{~g}^{1}
\end{aligned}
$$

LIGNIN, SOFTWOOD: solid; composition: carbon, $64 \%$; hydrogen, $6 \%$; oxygen, $30 \%$; empirical formula: $\mathrm{C}_{10} \mathrm{H}_{11}{ }_{2} \mathrm{O}_{3}, 3$; no general agreement prevails about the structure of lignin. Studies show that a dioxyphenylpropyl grouping is an important part of the polymer.

gross heat of combustion: assume values refer to room temperature.

$q_{v}($ gross $)=6300 \mathrm{cal} \mathrm{g}^{-1}$
$q_{v}($ gross $)=26360 \mathrm{~J} \mathrm{~g}^{-1}$ 
LIGNITE; solid; lignite is also called Brown Coal; (sample 1) ultimate analysis: carbon, $68.32 \%$; hydrogen, $4.00 \%$; oxygen, $25.54 \%$; nitrogen, $1.42 \%$; sulfur, $0.72 \%$; proximate analysis: ash, $13.27 \%$; moisture, $23.30 \%$; volatile matter, $43.5 \%$; loss on air drying, 17.3\%; (sample 2) ultimate analysis: carbon, $63.30 \%$; hydrogen, $3.32 \%$; oxygen, $29.86 \%$; nitrogen, $1.35 \%$; sulfur, $2.17 \%$; proximate analysis: ash, $10.12 \%$; moisture, $31.37 \%$; volatile matter, $50.6 \%$; loss on ai. drying, $14.2 \%$.

gross of heat of combustion: assume values refer to room temperature.

(sample 1, ash free and moisture free)

$q_{v}($ gross $)=6132 \mathrm{cal} \mathrm{g}^{-1}$
$q_{v}($ gross $)=25656 \mathrm{~J} \mathrm{~g}^{-1}$

(sample 1 , as received)

$q_{v}($ gross $)=4503 \mathrm{cal} \mathrm{g}^{-1}$
$q_{v}($ gross $)=18841 \mathrm{~J} \mathrm{~g}^{1}$

(sample 2, ash free and moisture free)

$q_{v}($ gross $)=5353 \mathrm{cal} \mathrm{g}^{-1}$
$q_{v}($ gross $)=22399 \mathrm{~J} \mathrm{~g}^{-1}$

(sample 2, as received)

$q_{v}($ gross $)=3132 \mathrm{cal} \mathrm{g}^{-1}$
$q_{v}($ gross $)=13104 \mathrm{~J} \mathrm{~g}^{1}$

LUNAR MATERIAL; solid; (Sample 10057) $\mathrm{SiO}_{2}, 36 \% ; \mathrm{Al}_{2} \mathrm{O}_{3}, 11 \% ; \mathrm{TiO}_{2}, 12.5 \%$; $\mathrm{Fe} 0,20 \%$; $\mathrm{MgO}, 9.5 \% ; \mathrm{CaO}, 10 \% ; \mathrm{Na}_{2} \mathrm{O}, 0.59 \% ; \mathrm{K}_{2} \mathrm{O}, 0.064 \% ; \mathrm{MnO}_{2}, 0.32 \% ; \mathrm{Cr}_{2} \mathrm{O}_{3}, 0.95 \%$; vesicular basait.

specific heat: 90 to $200 \mathrm{~K}$

$\begin{aligned} \operatorname{Cp}\left(\text { cal g } \mathrm{g}^{-1} \mathrm{~K}^{-1}\right) & =0.04880-5.476 \times 10^{-4} \mathrm{~T} \\ & +9.030 \times 10^{-6} \mathrm{~T}^{2}-2.074 \times 10^{-8} \mathrm{~T}^{3}\end{aligned}$

$\begin{aligned} C p\left(\mathrm{~J} \mathrm{~g}^{-1} \mathrm{~K}^{-1}\right) & =0.20418-2{ }^{2} 291 \times 10^{-3} \mathrm{~T} \\ & +3.778 \times 10^{-5} \mathrm{~T}^{2}-8.678 \times 10^{-8} \mathrm{~T}^{3}\end{aligned}$

200 to $350 \mathrm{~K}$

$$
\begin{aligned}
\operatorname{cp}\left(\operatorname{cal} g^{-1} K^{-1}\right) & =-0.03797+1-248 \times 10^{-3} \mathrm{~T}-2.277 \times 10^{-6} \mathrm{~T}^{2} \\
& +1.743 \times 10^{-9} \mathrm{~T}^{3} \\
\operatorname{cp}\left(\mathrm{J} \mathrm{g} \mathrm{g}^{-1} \mathrm{~K}^{-1}\right) & =-0.15887+5.222 \times 10^{-3} \mathrm{~T} \\
& -9.527 \times 10^{-6} \mathrm{~T}^{2}+7.293 \times 10^{-9} \mathrm{~T}^{3}
\end{aligned}
$$


LUNAR MATERIAL; solid; (Sample 10084) $\mathrm{SiO}_{2}, 41.86 \% ; \mathrm{TiO}_{2}, 7.56 \% ; \mathrm{Al}_{2} \mathrm{O}_{3}, 13.55 \%$; $\mathrm{Fe} 0$,

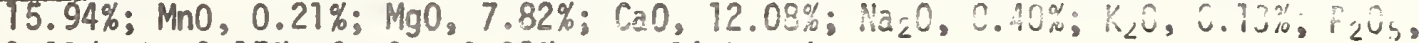
$0.11 \% ; \mathrm{S}, 0.15 \% ; \mathrm{Cr}_{2} \mathrm{O}_{3}, 0.32 \%$; regolith soil.

specific heat: 90 to $200 \mathrm{~K}$

$$
\begin{aligned}
\operatorname{Cp}\left(\operatorname{cal~g} g^{-1} K^{-1}\right) & =0.08584-1,343 \times 10^{-3} \mathrm{~T} \\
& +1.514 \times 10^{-5} \mathrm{~T}^{2}-3.620 \times 10^{-8} \mathrm{~T}^{3} \\
\operatorname{Cp}\left(\mathrm{J} \mathrm{g}^{-1} \mathrm{~K}^{-1}\right) & =0.35915-55619 \times 10^{-3} \mathrm{~T} \\
& +6.335 \times 10^{-5} \mathrm{~T}^{2}-1.515 \times 10^{-7} \mathrm{~T}^{3}
\end{aligned}
$$

200 to $350 \mathrm{~K}$

$$
\begin{aligned}
C p\left(\text { cal g } g^{-1} K^{-1}\right) & =0.03706+5,331 \times 10^{-4} \mathrm{~T} \\
& -1.273 \times 10^{-\xi} \mathrm{T}^{2}-3.131 \times 10^{-10} \mathrm{~T}^{3} \\
C_{p}\left(\mathrm{~g}^{-1} \mathrm{~K}^{-1}\right) & =0.15506+2,230 \times 10^{-3} \mathrm{~T} \\
& -5.326 \times 10^{-9} \mathrm{~T}^{2}-1.310 \times 10^{-9} \mathrm{~T}^{3}
\end{aligned}
$$

MANURE, PIG; solid; faeces from hogs; oven dried; $0 \%$ moisture.

gross heat of combustion: assume values refer to room temperature.

$q_{v}$ (gross) $=4280 \mathrm{cal} \mathrm{g}_{1}^{-1}$
$q_{v}$ (gross) $=17900 \mathrm{~J} \mathrm{~g}^{-1}$

NAPHTHA, ALASKA; 1iquid; U1timate analysis: carbon, $86.3 \%$; hydrogen, 13.7\%; nitrogen, $0.3 \mathrm{ppm}$; sulfur, $99 \mathrm{ppm}$, total paraffins, $39.7 \%$; total naphthenes, $39.6 \%$; total aromatics, $20.5 \%$; density, $0.7703 \mathrm{~g} \mathrm{~cm}^{-3}$ at $24{ }^{\circ} \mathrm{C}$; API gravity, 50.5 .

gross heat of combustion: assume values refer to room temperature.

$q_{v}($ gross $)=10841 \mathrm{cal}^{-1}$
$q_{v}($ gross $)=45360 \mathrm{~J} \mathrm{~g}^{-1}$

NAPHTHA, AROMATIC; 1iquid; UI timate analysis: carbon, $90.1 \%$; hydrogen, 9.8\%; sulfur, $760 \mathrm{ppm}$; total paraffins, $0.0 \%$; total naphthenes, $0.0 \%$; total aromatics, $83.1 \%$; total olefins, $16.9 \%$; density, $0.8449 ; \mathrm{g} \mathrm{cm}^{-3}$ at $24{ }^{\circ} \mathrm{C}$; API gravity, 34.5 . gross heat of combustion: assume values refer to room temperature.

$q_{v}($ gross $)=10287 \mathrm{cal} \mathrm{g}^{-1}$
$q_{v}($ gross $)=43040 \mathrm{~J} \mathrm{~g}^{-1}$ 
NAPHTHA, HIGH BOILING; 1iquid; U1timate analysis: carbon, $85.4 \%$; hydrogen, $14.6 \%$; sulfur, $357 \mathrm{ppm}$; total paraffins, $59.3 \%$; total naphthenes, $30.8 \%$; total aromatics, $9.9 \%$; density, $0.7559 ; \mathrm{g} \mathrm{cm}^{-3}$ at $24{ }^{\circ} \mathrm{C}$; API gravity, 54.2 .

gross heat of combustion: assume values refer to room temperature.

$q_{v}($ gross $)=11217 \mathrm{cal} \mathrm{g}^{-1}$
$q_{v}($ gross $)=46934 \mathrm{~J} \mathrm{~g}^{-l^{2}}$

NAPHTHA, JET; 1iquid; U1timate analysis: carbon, $86.05 \%$; hydrogen, $13.95 \%$; sulfur, $132 \mathrm{ppm}$; total paraffins, $18.6 \%$; total naphthenes, $69.8 \%$; total aromatics, $10.6 \%$; sulfur compounds, $1.0 \%$; density, $0.7981 ; \mathrm{g} \mathrm{cm}^{-3}$ at $24{ }^{\circ} \mathrm{C}$; API gravity, 44.4 .

gross heat of combustion: assume values refer to room temperature.

$q_{v}($ gross $)=11025 \mathrm{cal}^{-\mathrm{g}^{-1}}$
$q_{v}($ gross $)=46129 \mathrm{~J} \mathrm{~g}^{-l^{2}}$

NAPHTHA, LOW BOILING; 1iquid; U1timate analysis: carbon, $85.1 \%$; hydrogen, $14.9 \%$; sulfur, $376 \mathrm{ppm}$; total paraffins, $61.9 \%$; total naphthenes, $30.6 \%$; total aromatics, $7.6 \%$; density, 0.7559; $\mathrm{g} \mathrm{cm}^{-3}$ at $24{ }^{\circ} \mathrm{C}$; API gravity, 59.9.

gross heat of combustion: assume values refer to room temperature.

$q_{v}($ gross $)=11265 \mathrm{cal}^{-g^{-1}}$
$q_{v}($ gross $)=47132 \mathrm{Jg}^{-l^{2}}$

OIL, APIEZON C; liquid; low vapor-pressure residues of paraffin oil distillation products; average molecular weight $450 \pm 70$.

heat of vaporization: $40^{\circ}$ to $160^{\circ} \mathrm{C}$

$I_{v}=60.25 \mathrm{cal}_{v}=252.1 \mathrm{Jg}^{-1}$

vapor pressure: $40^{\circ}$ to $160^{\circ} \mathrm{C}$

$\log _{10} P($ Torr $)=11.67-5925 \mathrm{~T}^{-1}$
$\log _{10} \mathrm{P}(\mathrm{Pa})=13.79-5925 \mathrm{~T}^{-1}$ 
OIL, APIEZON E; liquid; IOW vapor-pressure residues of paraffin oil distillation products; average molecular weight $290 \pm 30$.

heat of vaporization: $40^{\circ}$ to $160^{\circ} \mathrm{C}$

$L_{v}=80.47 \mathrm{car}_{v} \mathrm{~g}^{-1}$
$L_{v}=336.7 \mathrm{~J} \mathrm{~g}^{-1}$

vapor pressure: $40^{\circ}$ to $160^{\circ} \mathrm{C}$

$\log _{10} P($ Torr $)=9.30-5100 \mathrm{~T}^{-1}$

$\log _{10} \mathrm{P}(\mathrm{Pa})=11.42-5100 \mathrm{~T}^{-1}$

OIL, CASTOR; liquid, oil from seeds of the castor bean, Ricinus communis; chief constituent: glyceride of ricinoleic acid (12-hydroxyoleic acid); saponification no. is 175-183; iodine no. is 83 after heating at $100{ }^{\circ} \mathrm{C}$ for 3 to 6 hours at 2-3 mm pressure; density is $0.945-0.965 \mathrm{~g} \mathrm{~cm}^{-3}$ at $25{ }^{\circ} \mathrm{C}$.

specific heat: $25^{\circ}$ to $275^{\circ} \mathrm{C}$

$\operatorname{Cp}\left(\right.$ cal $\left.g^{-1} K^{-1}\right)=-1.265+0.01241 T-2.8650 \times 10^{-5} T^{2}+2.264 \times 10^{-8} T^{3}$
$\operatorname{Cp}\left(J g K^{-1}\right)=-5.292+0.05192 T-1.1987 \times 10^{-4} T^{2}+9.474 \times 10^{-8} T^{3}$ 
OIL, CRUDE; 1iquid; ADMEG, Zakum (export), specific gravity $60 / 60^{\circ} \mathrm{F}=0.8256$, upper pour point $=-15{ }^{\circ} \mathrm{C}$, wax content $=7.0 \mathrm{wt}$. \%: Barrow Island, specific gravity. $60 / 60^{\circ} \mathrm{F}=0.8399$, upper pour point less than $-50^{\circ} \mathrm{C}$, wax content less than 1 wt. \%: Libyan (Tobruk export), specific gravity $60 / 60^{\circ} \mathrm{F}=0.8429$, upper pour point $=24{ }^{\circ} \mathrm{C}$, wax content $=20.0$ wt. \%: Iranian Light (export), specific gravity $60 / 60^{\circ} \mathrm{F}=0.8568$, upper pour point $=-21{ }^{\circ} \mathrm{C}$, wax content $=7.0$ wt. $\%$ : Kuwait (export), specific gravity $60 / 60^{\circ} \mathrm{F}=0.8709$, upper pour point $=-32{ }^{\circ} \mathrm{C}$, wax content $=5.5 \mathrm{wt}$. \%: Iranian Heavy (export), specific gravity $60 / 60^{\circ} \mathrm{F}=$ 0.8732 , upper pour point $=-12{ }^{\circ} \mathrm{C}$, wax content $=6.7$ wt. \%: Alaskan (North slope), specific gravity $60 / 60^{\circ} \mathrm{F}=0.8914$, upper pour point $=-1{ }^{\circ} \mathrm{C}$, wax content $=6.5 \mathrm{wt} . \%$.

gross heat of combustion: assume values refer to room temperature.

$q_{v}$ (gross) $=12400-2100 \mathrm{~d}^{2} \mathrm{cal} \mathrm{g}^{-1}$

$q_{v}^{v}($ gross $)=51881.6-8786.4 d^{2} \mathrm{~J} \mathrm{~g}^{-1}$

where $d$ is the specific gravity at $60 / 60^{\circ} \mathrm{F}$, equation assumes crude oil to be water, ash, and sulfur free; combustion products are gaseous $\mathrm{CO}_{2}$ and liquid $\mathrm{H}_{2} \mathrm{O}$.

$q_{v}{ }^{\prime}($ gross $)=q_{v}$ (gross) $-0.01 q_{v}$ (gross) $x+22.5(\stackrel{\circ}{\circ} s) \mathrm{cal} \mathrm{g}^{-1}$
$q_{v}{ }^{\prime}($ gross $)=q_{v}($ gross $)-0.04 q_{v}$ (gross) $x+94.1(\% 5) \mathrm{J} \mathrm{g}^{2}$

where $q_{y}{ }^{\prime}$ (gross) is heat of combustion at constant volume of crude oil yielding ash, gaseous $\mathrm{CO}_{2}, \mathrm{SO}_{2}$, and 1 iquid $\mathrm{H}_{2} \mathrm{O}$ as combustion products; $X=\left(\% \mathrm{H}_{2} \mathrm{O}+\% \mathrm{ash}+\% \mathrm{~S}\right)$

isothermal compressibility: $10^{5} \mathrm{bar}=1 \mathrm{~Pa}$

ADMEG/Zakum/(export)

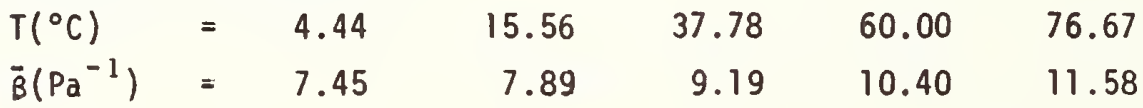

Barrow Island

\begin{tabular}{|c|c|c|c|c|}
\hline$\left.{ }^{\circ} \mathrm{C}\right)$ & $=$ & 4.44 & 15.56 & 37.78 \\
\hline$\left(\mathrm{Pa}^{-]}\right)$ & $=$ & 7.28 & 7.83 & 9.06 \\
\hline
\end{tabular}

Libyan (Tobruk export)

\begin{tabular}{|c|c|c|c|c|}
\hline$\left({ }^{\circ} \mathrm{C}\right)$ & $=$ & 37.78 & 50.00 & 60.00 \\
\hline$\left(\mathrm{Pa}^{-1}\right)$ & $=$ & 8.59 & 8.92 & 9.41 \\
\hline
\end{tabular}

Iranian Light (export)

\begin{tabular}{|c|c|c|c|c|c|c|}
\hline $\mathrm{T}\left({ }^{\circ} \mathrm{C}\right)$ & $=$ & 4.44 & 15.56 & 37.78 & 60.00 & 76.67 \\
\hline $\bar{B}\left(\mathrm{~Pa}^{-1}\right)$ & $=$ & 6.71 & 7.14 & 8.26 & 9.62 & 10.6 \\
\hline
\end{tabular}

Iranian Heavy (export)

\begin{tabular}{|c|c|c|c|c|c|c|}
\hline $\mathrm{T}\left({ }^{\circ} \mathrm{C}\right)$ & $=$ & 4.44 & 15.56 & 37.78 & 60.00 & 76.67 \\
\hline $\bar{B}\left(\mathrm{~Pa}^{-1}\right)$ & $=$ & 6.37 & 6.90 & 8.04 & 9.11 & 10.10 \\
\hline
\end{tabular}


Kuwait (export)

\begin{tabular}{|c|c|c|c|c|c|c|}
\hline$T\left({ }^{\circ} \mathrm{C}\right)$ & $=$ & 4.44 & 15.56 & 37.78 & 60.00 & 76.67 \\
\hline $\bar{\beta}\left(\mathrm{Pa}^{-1}\right)$ & $=$ & 6.46 & 6.83 & 8.03 & 9.06 & 10.20 \\
\hline
\end{tabular}

Alaskan (North Slope)

\begin{tabular}{|c|c|c|c|c|c|c|}
\hline $\mathrm{T}\left({ }^{\circ} \mathrm{C}\right)$ & $=$ & 4.44 & 15.56 & 37.78 & 60.00 & 76.67 \\
\hline $\bar{B}\left(\mathrm{~Pa}^{-1}\right)$ & $=$ & 6.18 & 6.22 & 7.25 & 8.26 & 9.11 \\
\hline
\end{tabular}

$[130]$

specific heat: The equation provided is based on over 100 measurements made at the National Bureau of Standards over the temperature range 0 to $204{ }^{\circ} \mathrm{C}$. The experimental results differ from the values calculated from this equation by less than $2 \%$, with a maximum deviation of $4 \%$; oils from mixed base crudes are in good agreement; olls from paraffin base crudes are systematically high by about $2 \%$; olls from naphthene base crudes are systernatically low by about $2 \%$.

$d$ is the specific gravity at $60 \% / 60^{\circ} \mathrm{F}$,

$\operatorname{Cp}\left(\operatorname{cal} g^{-1} K^{-1}\right)=\left(0.1811+8.1 \times 10^{-4} \mathrm{~T}\right) / \mathrm{d}^{\frac{1}{2}}$
$\operatorname{Cp}\left(J \mathrm{~J}^{-1} \mathrm{~K}^{-1}\right)=\left(0.7577+3.39 \times 10^{-3} \mathrm{~T}\right) / \mathrm{d}^{\frac{1}{2}}$

OIL, FUEL; 1iquid; Ultimate analysis: carbon, $86.9 \%$; hydrogen, $13.1 \%$; sulfur, $172 \mathrm{ppm}$; total paraffins, $29.8 \%$; total naphthenes, $45.6 \%$; total aromatics, $22.4 \%$; sulfur compounds, $1.3 \%$; density, $0.8553 ; \mathrm{g} \mathrm{cm}^{-3}$ at $24{ }^{\circ} \mathrm{C}$; API gravity 33.0 .

gross heat of combustion: assume values refer to room temperature.

$q_{v}($ gross $)=9763 \mathrm{cal} \mathrm{g}^{-1}$
$q_{v}($ gross $)=40847 \mathrm{~J} \mathrm{~g}^{2}$

OIL, GAS; liquid; U1timate analysis: carbon, $86.5 \%$; hydrogen, $13.4 \%$; sulfur, 261 ppm: nitrogen, $3 \mathrm{ppm}$; total paraffins, $38.8 \%$; total naphthenes, $41.5 \%$; total aromatics, 17.2\%; sulfur compounds, $2.4 \%$; density, $0.8415 ; \mathrm{g} \mathrm{cm}^{-3}$ at $24{ }^{\circ} \mathrm{C}$; API gravity, 35.3 .

gross heat of combustion: assume values refer to room temperature.

$q_{v}$ (gross) $=10977 \mathrm{cal}^{-g^{-1}}$
$q_{v}$ (gross) $=45927 \mathrm{Jg}^{-1}$ 
OIL, LEYBOLD F; liquid; a mixture of hydrocarbons prepared by Leybold Köln; average molecular weight $360 \pm 50$.

heat of vaporization: temperature range $40^{\circ}$ to $160^{\circ} \mathrm{C}$.

$L_{v}=69.02 \mathrm{call}^{-1}$
$L_{v}=288.8 \mathrm{~J} \mathrm{~g}^{-1}$

vapor pressure: temperature range $40^{\circ}$ to $160^{\circ} \mathrm{C}$.

$\log _{10} P($ Torr $)=10.43-5430 \mathrm{~T}^{-1}$

$\log _{10} \mathrm{P}(\mathrm{Pa})=12.55-5430 \mathrm{~T}^{-1}$

OIL, LEYBOLD H; liquid; a mixture of hydrocarbons prepared by Leybold Köln; average molecular weight $385 \pm 40$.

heat of vaporization: temperature range $40^{\circ}$ to $160^{\circ} \mathrm{C}$.

$L_{v}=70.12 \mathrm{cal}_{v}=293.4 \mathrm{~J} \mathrm{~g}^{-1}$
$L_{v}$

vapor pressure: tenperature range $40^{\circ}$ to $160^{\circ} \mathrm{C}$.

$\log _{10} P($ Torr $)=11.82-5900 \mathrm{~T}^{-1}$

$\log _{10} P(P a)=13.95-5900 \mathrm{~T}^{-1}$

OIL, LINSEED; liquid; (flaxseed oil); obtained from dried seeds of flax, Linum usitatissiumum (Linaceae); chief constituents are glycerides of Tinoleic $(261.5 \%)$, linolenic $(225.0 \%)$, palmitic $(25 \%)$, oleic $(25 \%)$, and stearic $(\sim 3.5 \%)$ acids; iodine no. 172.1 , density $0.930-0.938 \mathrm{~g} \mathrm{~cm}^{-3}$.

specific heat: sample was heated at $100{ }^{\circ} \mathrm{C}$ for 3 to 6 hours under a pressure of $2-3 \mathrm{~mm}$ of $\mathrm{Hg}$ to remove moisture; equations valid 30 to $270{ }^{\circ} \mathrm{C}$.

$$
\begin{aligned}
\mathrm{Cp}\left(\mathrm{cal} \mathrm{g} \mathrm{g}^{-1} \mathrm{~K}^{-1}\right)= & -0.4606+6.4288 \times 10^{-3} \mathrm{~T} \\
& -1.4922 \times 10^{-5} \mathrm{~T}^{2}+1.2492 \times 10^{-8} \mathrm{~T}^{3} \\
\mathrm{Cp}\left(\mathrm{J} \mathrm{g}^{-1} \mathrm{~K}^{-1}\right)= & -1.9272+2.6898 \times 10^{-2} \mathrm{~T} \\
& -6.2434 \times 10^{-5} \mathrm{~T}^{2}+5.2267 \times 10^{-8} \mathrm{~T}^{3}
\end{aligned}
$$

OIL, LUBRICATING, P-10; liquid; chief ingredient is di-(2-ethylhexyl) sebacate $\left[\mathrm{C}_{22} \mathrm{H}_{50} \mathrm{O}_{4}\right]$, other ingredients are Plexol 201, rust inhibitor, and antioxidant.

vapor pressure: $20-108{ }^{\circ} \mathrm{C}$

$\log _{10} P($ Torr $)=7.23-4109 \mathrm{~T}^{-1}$
$\log _{10} \mathrm{P}(\mathrm{Pa})=9.36-4109 \mathrm{~T}^{-1}$ 
OIL, NEATSFOOT; Tiquid; paie yellow oil obtained by boiling the shin bones and feet (hoofs removed) of cattle in water; oil is separated from fat obtained.

specific heat: $25^{\circ} \mathrm{C}$

$\operatorname{Cp}\left(\operatorname{cal} g^{-1} K^{-1}\right)=0.5191$
$\operatorname{Cp}\left(J g^{-1} K^{-1}\right)=2.1719$

OIL, PARAFFIN; 1iquid; repeating unit: $\mathrm{CH}_{1.891}$.

gross heat of combustion: $25{ }^{\circ} \mathrm{C}$

$q_{v}($ gross $)=10984 \mathrm{cal} \mathrm{g}^{-1}$
$q_{v}($ gross $)=45957 \mathrm{~J} \mathrm{~g}^{-1}$

OIL, PERILLA; li quid; obtained from seeds of Perilla ocimoides; chief constituents are glycerides of linoleic ( $39 \%)$ linolenic ( $246 \%)$ acids, with small amounts of palmitic $(28 \%)$ and oleic $(8 \%)$ acids; density $0.93-0.94 \mathrm{~g} \mathrm{~cm}^{-3}$; before measurement oil was heated at $100{ }^{\circ} \mathrm{C}$ for 3 to 6 hours at $2-3 \mathrm{~mm}$ of $\mathrm{Hg}$ to remove moisture; iodine no. 186.2 .

specific heat: $6^{\circ}$ to $270^{\circ} \mathrm{C}$

$\operatorname{Cp}\left(\operatorname{cal} g^{-1} K^{-1}\right)=0.4082-2.410 \times 10^{-4} T+1.000 \times 10^{-6} T^{2}$
$\operatorname{Cp}\left(J g^{-1} K^{-1}\right)=1.7081-1.008 \times 10^{-3} T+4.184 \times 10^{-6} T^{2}$

OIL, TUNG; liquid; a yellow drying oil from the nuts of the trees Aleurites cordata and Aleurites fordii; glycerides of eleostearic ( $280 \%)$; palmitic ( $24 \%)$; stearic ( $1 \%)$; and oleic $(215 \%)$ acids; density $0.936-0.942 \mathrm{~g} \mathrm{~cm}^{-3}$; before measurement oil was heated at $100{ }^{\circ} \mathrm{C}$ for 3 to 6 hours at $2-3 \mathrm{~mm}$ of $\mathrm{Hg}$ pressure to remove moisture; iodine no. 154.4 .

specific heat: $20^{\circ}$ to $200^{\circ} \mathrm{C}$

$$
\begin{aligned}
\operatorname{CP}\left(\operatorname{cal~} \mathrm{g}^{-1} \mathrm{~K}^{-1}\right)= & -0.6053+7.0447 \times 10^{-3} \mathrm{~T} \\
& -1.5461 \times 10^{-5} \mathrm{~T}^{2}+1.2178 \times 10^{-8} \mathrm{~T}^{3} \\
\operatorname{CP}\left(\mathrm{J} \mathrm{g}^{-1} \mathrm{~K}^{-1}\right)= & -2.5324+2.9475 \times 10^{-2} \mathrm{~T} \\
& -6.4687 \times 10^{-5} \mathrm{~T}^{2}+5.0954 \times 10^{-8} \mathrm{~T}^{3}
\end{aligned}
$$


PAPER, BROWN; Solid; Ultimate analysis in percent by weight; carbon, $44.90 \%$; hycirogen, $6.08 \%$; oxygen, $47.84 \%$; nitrogen, $0.00 \%$; sulfur, $0.11 \%$; ash, $1.07 \%$; proximate analysis as received in percent by weight; moisture, $5.83 \%$; volatile matter, $83.92 \%$; fixed carbon, $9.24 \%$; ash, $1.01 \%$.

gross heat of combustion: assume values refer to room temperature.

(as received)

$q_{v}($ gross $)=4034 \mathrm{cal} \mathrm{g}^{-1}$
$q_{v}($ gross $)=16877 \mathrm{~J} \mathrm{~g}^{1}$

(dry basis)

$q_{v}($ gross $)=4284 \mathrm{cal} \mathrm{g}^{-1}$
$q_{v}($ gross $)=17924 \mathrm{~J} \mathrm{~g}^{1}$

[62]

PAPER, CORRUGATED BOX; solid; U1timate analysis in percent by weight; carbon, $43.73 \%$; hydrogen, 5.70; oxygen, $44.93 \%$; nitrogen, $0.09 \%$; sulfur, $0.21 \%$; ash, $5.34 \%$; proximate analysis as received in percent by weight; moisture, $5.20 \%$; volatile matter, $77.47 \%$; fixed carbon, $12.27 \%$; ash, $5.06 \%$.

gross heat of combustion: assume values refer to room temperature.

(as received)

$q_{v}($ gross $)=3915 \mathrm{cal} \mathrm{g}^{-1}$
$q_{v}($ gross $)=16382 \mathrm{~J} \mathrm{~g}^{2}$

(dry basis)

$q_{v}($ gross $)=4130 \mathrm{cal} \mathrm{g}^{-1}$
$q_{v}($ gross $)=17280 \mathrm{~J} \mathrm{~g}^{1}$

[62]

PAPER, FOOD CARTONS; solid; Ultimate analysis in percent by weight; carbon, $44.74 \%$;

hydrogen, $6.10 \%$; oxygen, $41.92 \%$; nitrogen, $0.15 \%$; sulfur, $0.16 \%$; ash, $6.93 \%$;

proximate analysis as received in percent by weight; moisture, $6.11 \%$;

volatile matter, $75.59 \%$; fixed carbon, $11.80 \%$; ash, $6.50 \%$.

gross heat of combustion: assume values refer to room temperature.

(as received)

$q_{v}($ gross $)=4035 \mathrm{cal} \mathrm{g}^{-1}$
$q_{v}($ gross $)=16882 \mathrm{~J} \mathrm{~g}^{1}$

(dry basis)

$q_{v}($ gross $)=4297 \mathrm{cal} \mathrm{g}^{-1}$
$q_{v}($ gross $)=17980 \mathrm{~J} \mathrm{~g}^{1}$ 
PAPER, JUNK MAIL; solid; UItimate analysis in percent by weignt; carbon, $51.81 \%$; nyarogen, $5.47 \%$; oxygen, $42.74 \%$; nitrogen, $0.17 \%$; sulfur, $0.09 \%$; ash, $13.72 \%$; proximate analysis as received in percent by weight; moisture, $4.56 \%$; volatile matter, 73. $32 \%$; fixed carbon, $9.03 \%$; ash, $13.09 \%$.

gross heat of combustion: assume values refer to room temperature.

(as received)

$q_{v}($ gross $)=3385 \mathrm{cal} \mathrm{g}^{-1}$
$q_{v}($ gross $)=14161 \mathrm{Jg}^{-1}$

(dry basis)

$q_{v}$ (gross) $=3546 \mathrm{cal} \mathrm{g}^{-1}$
$q_{v}$ (gross) $=14835 \mathrm{~J} \mathrm{~g}^{1}$

PAPER, MAGAZINE; solid; U1timate analysis in percent by weight; carbon, $32.91 \%$; hydrogen, 4.95\%; oxygen, $38.55 \%$; nitrogen, $0.07 \%$; sulfur, $0.09 \%$; ash, $23.43 \%$; proximate analysis as received in percent by weight; moisture, $4.11 \%$; volatile matter, $66.39 \%$; fixed carbon, $7.03 \%$; ash, $22.47 \%$.

gross heat of combustion: assume values refer to room temperature.

(as received)

$q_{v}($ gross $)=2921 \mathrm{cal} \mathrm{g}^{-1}$
$q_{v}($ gross $)=12221 \mathrm{~J} \mathrm{~g}^{2}$

(dry basis)

$q_{v}($ gross $)=3046 \mathrm{cal} \mathrm{g}^{-1}$
$q_{v}($ gross $)=12746 \mathrm{~J} \mathrm{~g}^{-1}$

PAPER, NEWSPRINT; solid; U1timate analysis in percent by weight; carbon, 49.14\%; hydrogen, $6.10 \%$; oxygen, $43.03 \%$; nitrogen, $0.05 \%$; sulfur, $0.16 \%$; ash, $1.52 \%$; proximate analysis as received in percent by weight; moisture, $5.97 \%$; volatile matter, $81.12 \%$; fixed carbon, $11.48 \%$; ash, $1.43 \%$.

gross heat of combustion: assume values refer to room temperature.

(as received)

$q_{v}($ gross $)=4433 \mathrm{cal} \mathrm{g}^{-1}$
$q_{v}($ gross $)=18548 \mathrm{~J} \mathrm{~g}^{2}$

(dry basis)

$q_{v}($ gross $)=4714 \mathrm{cal} \mathrm{g}^{-1}$
$q_{v}($ gross $)=19724 \mathrm{~J} \mathrm{~g}_{1}$ 
PAPER, PLASTIC COATED; solid; Ultimate analysis in percent by weight; carbon, $45.30 \%$; hydrogen, $6.17 \%$; oxygen, $45.50 \%$; nitrogen, $0.18 \%$; sulfur, $0.08 \%$; ash, $2.77 \%$; proximate analysis as received in percent by weight; moisture, $4.71 \%$; volatile matter, 84.20\%; fixed carbon, $8.45 \%$; ash, $2.64 \%$.

gross heat of combustion: assume values refer to room temperature.

(as received)

$q_{v}($ gross $)=4081 \mathrm{cal} \mathrm{g}^{-1}$
$q_{v}($ gross $)=17075 \mathrm{~J} \mathrm{~g}^{-1}$

(dry basis)

$q_{v}($ gross $)=4282 \mathrm{cal} \mathrm{g}^{-1}$
$q_{v}($ gross $)=17917 \mathrm{~J} \mathrm{~g}^{1}$

PAPER, WAXED MILK CARTONS; solid; Ultimate analysis in percent by weight; carbon, $59.18 \%$; hydrogen, $9.25 \%$; oxygen, $30.13 \%$; nitrogen, $0.12 \%$; sulfur, $0.10 \%$; ash, $1.22 \%$; proximate analysis as received in percent by weight; moisture, $3.45 \%$; volatile matter, $90.92 \%$; fixed carbon, $4.46 \%$; ash, $1.17 \%$.

gross heat of combustion: assume values refer to room temperature.

(as received)

$q_{v}($ gross $)=6297 \mathrm{cal} \mathrm{g}^{-1}$
$q_{v}($ gross $)=26347 \mathrm{~J} \mathrm{~g}^{1}$

(dry basis)

$q_{v}($ gross $)=6522 \mathrm{cal} \mathrm{g}^{-1}$
$q_{v}($ gross $)=27289 \mathrm{~J} \mathrm{~g}^{2}$ 
PEAT; solid; highly organic soil containing partially decomposed vegetable matter and having more than $50 \%$ combustible content.

gross heat of combustion: assume values refer to room termperature.

sample 1; air dried; moisture, $8.68 \%$; volatile material; $50.92 \%$; fixed carbon, $23.79 \%$; ash, $16.61 \%$; sulfur, $0.99 \%$.

$q_{v}($ gross $)=4182 \mathrm{cal} \mathrm{g}^{-1}$
$q_{v}($ gross $)=17496 \mathrm{~J} \mathrm{~g}^{1}$

sample 2; brown fibrous; air dried; moisture, $6.34 \%$; ash, $7.93 \%$; sulfur, $0.69 \%$.

$q_{v}($ gross $)=5161 \mathrm{cal}^{-1}$

$q_{v}($ gross $)=21594 \mathrm{~J} \mathrm{~g}^{2}$

sample 3; black; air dried; moisture, $6.62 \%$; ash, $24.44 \%$; sulfur, $0.65 \%$.

$q_{v}($ gross $)=3992 \mathrm{cal} \mathrm{g}^{-1}$
$q_{v}($ gross $)=16703 \mathrm{~J} \mathrm{~g}^{1}$

sample 4; brown; air dried; moisture, $12.10 \%$; ash, $7.22 \%$; sulfur, $0.83 \%$.

$q_{v}($ gros $\dot{s})=4269 \mathrm{cal} \mathrm{g}^{-1}$
$q_{v}($ gross $)=17861 \mathrm{~J} \mathrm{~g}^{-1}$

\section{PLANTS}

ALGAE; solid; chlorophyl-bearing organisms occurring in both salt and fresh water; contain minerals, proteins, lipids, amino acids; a source of iodine.

gross heat of combustion: assume values refer to room temperature and are corrected for moisture and ash content.

Ulva rigida (green alga)

$q_{v}($ gross $)=3750 \mathrm{cal} \mathrm{g}^{-1}$
$q_{v}($ gross $)=15690 \mathrm{~J} \mathrm{~g}^{2}$

Alaria nana (brown)

$q_{v}($ gross $)=3250 \mathrm{cal} \mathrm{g}^{-1}$
$q_{v}($ gross $)=13598 \mathrm{~J} \mathrm{~g}^{-1}$

Fucus distichus (brown)

$q_{v}($ gross $)=3430 \mathrm{cal} \mathrm{g}^{-1}$
$q_{v}($ gross $)=14351 \mathrm{~J} \mathrm{~g}^{-1}$ 
GRASS, LAWN CLIPPINGS; solid; Ultimate analysis: carbon, $43.65 \%$; hydrogen, $6.74 \%$; oxygen, 33.33\%; nitrogen, $4.22 \%$; sulfur, $0.40 \%$; ash, $6.19 \%$; moisture, $5.47 \%$; proximate analysis as received: moisture, $5.47 \%$; volatile matter, $71.16 \%$; fixed carbon, $17.18 \%$; ash, $6.19 \%$.

gross heat of combustion: assume values refer to room temperature.

(air dried at $80^{\circ} \mathrm{C}$ )

$q_{v}($ gross $)=4369 \mathrm{cal} \mathrm{g}^{-1}$
$q_{v}($ gross $)=18277 \mathrm{~J} \mathrm{~g}^{1}$

(as received)

$q_{v}($ gross $)=1144 \mathrm{cal} \mathrm{g}^{-1}$
$q_{v}$ (gross) $=4787 \mathrm{~J} \mathrm{~g}^{-1}$

HERBS, ALPINE TUNDRA; solid; samples of 20 species of herbs were collected in the alpine tundra of Mt. Washington, New Hampshire. Mean lipid content was $1.43 \pm 0.1 \%$ of volatile-free dry weight. Samples ground and oven-dried at $80{ }^{\circ} \mathrm{C}$.

gross heat of combustion: assume values refer to room temperature and are corrected for moisture and ash content.

$q_{v}($ gross $)=4601 \pm 29 \mathrm{cal} \mathrm{g}^{-1}$
$q_{v}($ gross $)=19251 \pm 121 \mathrm{~J} \mathrm{~g}^{-1}$

LEAVES, TREE, RIPE HARDWOOD MIXTURE; solid; Ultimate analysis: carbon, $52.15 \%$; hydrogen, $6.11 \%$; oxygen, $30.34 \%$; nitrogen, $6.99 \%$; sulfur, $0.16 \%$; ash, $4.25 \%$; proximate analysis as received: moisture, $9.97 \%$; volatile matter, $66.92 \%$; fixed carbon, $19.29 \%$; ash, $3.82 \%$.

gross heat of combustion: assume values refer to room temperature.

(as received)

$q_{v}($ gross $)=4439 \mathrm{cal} \mathrm{g}^{-1}$
$q_{v}($ gross $)=18571 \mathrm{~J} \mathrm{~g}^{1}$

(dry basis)

$q_{v}($ gross $)=4930 \mathrm{cal} \mathrm{g}^{-1}$
$q_{v}($ gross $)=20629 \mathrm{~J} \mathrm{~g}^{1}$ 


\section{PLANTS}

MOSSES, ALPINE TUNDRA; solid; samples of 7 species of mosses were collected in the alpine tundra of Mt. Washington, New Hampshire. Mean lipid content was $1.0 \%$ of the volatile-free dry weight. Samples were ground and air-dried at $80{ }^{\circ} \mathrm{C}$.

gross heat of combustion: assume values refer to room temperature, and are corrected for moisture and ash content.

$q_{v}($ gross $)=4410 \pm 70 \mathrm{cal} \mathrm{g}^{-1}$
$q_{v}($ gross $)=18450 \pm 293 \mathrm{Jg}^{-1}$

PLANTS, FLOWER GARDEN; solid; Ul timate analysis: carbon, $46.65 \%$; hydrogen, $6.61 \%$;

oxygen, $40.18 \%$; nitrogen, $1.21 \%$; sulfur, $0.26 \%$; ash, $5.09 \%$; proximate analysis as received: moisture, $53.94 \%$; volatile matter, $35.64 \%$; fixed carbon, $8.08 \%$; ash, $2.34 \%$.

gross heat of combustion: assume values refer to room temperature.

(as received)

$q_{v}($ gross $)=2055 \mathrm{cal}^{-g^{-1}}$
$q_{v}($ gross $)=8599 \mathrm{~J} \mathrm{~g}^{-1}$

(dry basis)

$q_{v}($ gross $)=4462 \mathrm{cal} \mathrm{g}^{-1}$
$q_{v}($ gross $)=18671 \mathrm{~J} \mathrm{~g}^{2}$

PLANTS, VASCULAR AQUATIC; solid; plants taken from Par Pond, U.S. AEC Savannah River Plant, near Aiken, South Carolina; samples dried at $60{ }^{\circ} \mathrm{C}$ and pulverized to pass 40 mesh screen.

gross heat of combustion: assume values refer to room temperature.

Species

Typha latifolia Hydrotrida carolinensis

Brasenia schreberi Utricularia inflata Nelumbo lutea

Myriophyl lum heterophyllum Eleocharis acicularis Najas guadalupensis Nymphaea odorata Ceratophyllum demensum Nuphar advena
Protein Content g (100 g.dry wt.)

$\begin{array}{rll}4.0 & 4262 \mathrm{cal} \mathrm{g}^{-1} & 17832 \mathrm{~J} \mathrm{~g}^{-1} \\ 10.5 & 4058 & 16979 \\ 10.9 & 4026 & 16845 \\ 11.4 & 4023 & 16832 \\ 12.7 & 4227 & 17686 \\ 13.5 & 3961 & 16573 \\ 14.1 & 4256 & 17807 \\ 14.4 & 3918 & 16393 \\ 14.6 & 4180 & 17489 \\ 17.1 & 3906 & 16343 \\ 21.6 & 4315 & 18054\end{array}$

[113] 
SHRUBS, DECIDUOUS, ALPINE TUNDRA; solid; samples of 9 species of shrubs wert: collected in the alpine tundra of Mt. Washington, New Hampshire. Mean lipid content was $1.97 \pm 0.3 \%$ of the volatile-free dry weight. Samples were ground and ovendried at $80{ }^{\circ} \mathrm{C}$.

gross heat of combustion: assume values refer to room temperature, and are corrected for moisture and ash content.

$q_{v}($ gross $)=4932 \pm 33 \mathrm{cal} \mathrm{g}^{-1}$
$q_{v}($ gross $)=20635 \pm 138 \mathrm{~J} \mathrm{~g}^{-1}$

SHRUBS, EVERGREEN, ALPINE TUNORA; solid; samples of 11 species of shrubs were collected in the alpine tundra of Mt. Washington, New Hampshire. Mean lipid content is $3.72 \pm 0.5 \%$ of the volatile-free dry weight. Samples were ground and oven-dried at $80{ }^{\circ} \mathrm{C}$.

gross heat of combustion: assume values refer to room temperature, and are corrected for moisture and ash content.

$q_{v}$ (gross) $=5098 \pm 48 \mathrm{cal} \mathrm{g}^{-1}$
$q_{v}($ gross $)=21330 \pm 201 \mathrm{~J} \mathrm{~g}^{-1}$

SHRUBS, EVERGREEN, CUTTINGS; solid; Ul timate analysis: carbon, $48.51 \%$; hydrogen, $6.54 \%$; oxygen, $40.44 \%$; nitrogen, $1.71 \%$; sulfur, $0.19 \%$; ash, $2.61 \%$; proximate analys is as received: moisture, $69.00 \%$; volatile matter, $25.18 \%$; fixed carbon, $5.01 \%$; ash, $0.81 \%$.

gross heat of combustion: assume values refer to room temperature.

(as received)

$q_{v}($ gross $)=1505 \mathrm{cal}^{-g^{-1}}$
$q_{v}($ gross $)=6299 \mathrm{~J} \mathrm{~g}^{-1}$

(dry basis)

$q_{v}($ gross $)=4856 \mathrm{cal} \mathrm{g}^{-1}$
$q_{v}($ gross $)=20318 \mathrm{~J} \mathrm{~g}^{1}$ 


\section{POLYMERS}

BUTADIENE-STYRENE COPOLYMER, GL-622; solid; composition: carbon $90.100 \%$; hydrogen, $9.710 \%$; sulfur, $0.081 \%$; oxygen, $0.14 \%$; ash, $0.171 \%$; styrene, $42.98 \%$; phenyl-Bnaphthylamine $0.1 \%$; polymerized at $50{ }^{\circ} \mathrm{C}$.

specific heat:

Temperature $(K)$

5
50
100
150
200
220
255

$$
\begin{gathered}
\left.C p(c a) g^{-1} k^{-1}\right) \\
0.00081 \\
0.076 \\
0.136 \\
0.190 \\
0.244 \\
0.268 \\
0.405
\end{gathered}
$$

$$
\begin{gathered}
C p\left(J g^{-1} K^{-1}\right) \\
0.0034 \\
0.318 \\
0.569 \\
0.794 \\
1.022 \\
1.122 \\
1.694
\end{gathered}
$$

for temperature range: $255^{\circ}-330^{\circ} \mathrm{K}$

$C p\left(\operatorname{cal} g^{-1} K^{-1}\right)=0.316+4.37 \times 10^{-5} T+1.195 \times 10^{-6} \mathrm{~T}^{2}$

$C p\left(\mathrm{Jg}^{-1} \mathrm{~K}^{-1}\right)=1.322+1.83 \times 10^{-4} \mathrm{~T}+5.000 \times 10^{-6} \mathrm{~T}^{2}$

BUTADIENE-STYRENE COPOLYMER, X-454; solid; composition: carbon $88.869 \%$; hydrogen, $10.872 \%$; suTfur, $0.056 \%$; oxygen, $0.235 \%$; ash, $0.18 \%$; styrene, $8.58 \%$; phenyl- $\beta$-naphthylamine, $0.1 \%$; polymerized at $5{ }^{\circ} \mathrm{C}$.

specific heat:

$\begin{array}{ccc}\text { Temperature }(K) & \left.C p(c a) g^{-1} K^{-1}\right) & C p\left(J^{-1} K^{-1}\right) \\ 50 & 0.084 & 0.357 \\ 100 & 0.153 & 0.464 \\ 150 & 0.220 & 0.92 \\ 200 & 0.373 & 1.56 \\ 250 & 0.423 & 1.77 \\ 300 & 0.464 & 1.94 \\ 330 & 0.485 & 2.03\end{array}$


BUTADIENE-STYRENE COPOLYMER, $X-478$; solid; composition: carbon, $89.443 \%$; hydrugen, $10.4008 \%$; sulfur, $0.048 \%$; oxygen, $0.15 \%$; ash, $0.098 \%$; styrene, $22.61 \%$; pheny $1-\beta$-naphthylamine, $0.1 \%$; polymerized at $5{ }^{\circ} \mathrm{C}$.

specific heat:

Temperature $(K)$

15
50
100
150
195
225

15

150

195

225

$$
C p\left(\operatorname{cal} g^{-1} K^{-1}\right)
$$

0.016

0.081

0.147

0.209

0.262

0.405

$$
\operatorname{Cp}\left(J g^{-1} K^{-1}\right)
$$

0.067

0.338

0.617

0.874

1.096

1.693

225 to $330{ }^{\circ} \mathrm{K}$

$\left.C p(c a) g^{-1} K^{-1}\right)=0.3337+6.29 \times 10^{-5} T+1.1205 \times 10^{-6} \mathrm{~T}^{2}$

$C p\left(\mathrm{~J} \mathrm{~g}^{-1} \mathrm{~K}^{-1}\right)=1.396+2.632 \times 10^{-4} \mathrm{~T}+4.6882 \times 10^{-6} \mathrm{~T}^{2}$

BUTENE-1-POLYSULFONE; solid; repeating unit: $\mathrm{C}_{4} \mathrm{H}_{8} \mathrm{O}_{2} \mathrm{~S}$.

gross heat of combustion: at $25^{\circ} \mathrm{C}$

$q_{v}($ gross $)=5748.3 \pm 3.8 \mathrm{cal} \mathrm{g}^{-1}$
$q_{v}($ gross $)=24050.9 \pm 15.9 \mathrm{~J} \mathrm{~g}^{-1}$

Cis-BUTENE-2-POLYSULFONE; solid; repeating unit: $\mathrm{C}_{4} \mathrm{H}_{8} \mathrm{O}_{2} \mathrm{~S}$.

gross heat of combustion: at $25^{\circ} \mathrm{C}$.

$q_{v}($ gross $)=5744.6 \div 2.6 \mathrm{cal} \mathrm{g}^{-1}$
$q_{v}($ gross $)=24035.4+10.9 \mathrm{~J} \mathrm{~g}^{-1}$

tranS-BUTENE-2-POLYSULFONE; solid; repeating unit: $\mathrm{C}_{4} \mathrm{H}_{8} \mathrm{O}_{2} \mathrm{~S}$.

gross heat of combustion: at $25^{\circ} \mathrm{C}$.

$q_{v}($ gross $)=5745.7 \pm 2.5 \mathrm{cal} \mathrm{g}^{-1}$
$q_{v}($ gross $)=24040.0 \pm 10.5 \mathrm{~J} \mathrm{~g}^{-1}$ 
ISO-BUTENE POLYSULFONE (2-methylpropene polysulfone); solid; repeating unit: $\mathrm{C}_{4} \mathrm{H}_{8} \mathrm{O}_{2} \mathrm{~S}$. gross heat of combustion: at $25^{\circ} \mathrm{C}$.

$q_{v}($ gross $)=5753.2 \pm 2.9 \mathrm{cal}^{-1}$
$q_{v}($ gross $)=24071.4 \pm 12.1 \mathrm{~g}^{-1}$

DELRIN; solid; (polyoxymethylene); E.I. duPont de Nemours and Co. trade name; crystallized resin granules, $99.3 \%$; unspecified organic additives $0.7 \%$; repeating unit: $\mathrm{CH}, 0$.

gross heat of combustion: at $25^{\circ} \mathrm{C}$.

$q_{v}$ (gross) $=4047.7 \pm 0.8 \mathrm{cal} \mathrm{g}^{-1}$
$q_{v}$ (gross) $=16933.3 \pm 3.3 \mathrm{Jg}^{2}$

DICYANDIAMIDE (TECHNICAL); solid; elementary analysis; carbon, $28.97 \%$; hydrogen, $4.44 \%$; nitrogen, $66.60 \%$; formula $\mathrm{C}_{2} \mathrm{H}_{4} \mathrm{~N}_{4}$.

gross heat of combustion: at $25^{\circ} \mathrm{C}$.

unhardened

$q_{v}($ gross $)=3735.4 \pm 1.7 \mathrm{cal} \mathrm{g}^{-1}$
$q_{v}($ gross $)=15628.9 \pm 7.1 \mathrm{Jg}^{2}$

hardened

$q_{v}($ gross $)=3762.4 \pm 2.7 \mathrm{cal} \mathrm{g}^{-1}$
$q_{v}($ gross $)=15741.9 \pm 8.8 \mathrm{Jg}^{-1}$

DURETHAN U20; solid; a polyurethane from 1,4-butanediol and hexamethyldiisocyanate;

formula $\mathrm{C}_{12} \mathrm{H}_{22} \mathrm{O}_{4} \mathrm{~N}_{2}$.

gross heat of combustion: at $25^{\circ} \mathrm{C}$.

$q_{v}($ gross $)=6221.4 \pm 1.9 \mathrm{cal} \mathrm{g}^{-1}$
$q_{v}($ gross $)=26030.3 \pm 7.9 \mathrm{Jg}^{-1}$

EPILOX EGI. HARDENED; solid; empirical formula $\mathrm{C}_{39} \mathrm{H}_{40} \mathrm{O}_{8} .5$; the unhardened resin plus phthal ic anhydride.

gross heat of combustion: at $25^{\circ} \mathrm{C}$.

$q_{v}($ gross $)=7233.9 \pm 1.9 \mathrm{cal} \mathrm{g}^{-1}$
$q_{v}($ gross $)=30266.6 \pm 7.9 \mathrm{Jg}^{-1}$ 
EPILOX EGI, UNHARDENED; solid; elementary analysis; carbon, $74.74 \%$; hydrogen, $7.27 \%$; oxygen, 17.99\%; a diepoxide from epichlorhydrin plus 4, 4'-dihydroxydiphenyl-2,2' propane; empirical formula $\mathrm{C}_{31} \mathrm{H}_{36} \mathrm{O}_{5.5}$.

gross heat of combustion: at $25{ }^{\circ} \mathrm{C}$.

$q_{v}($ gross $)=7867.0 \pm 2.5 \mathrm{cal} \mathrm{g}^{-1}$
$q_{v}($ gross $)-32915.5 \pm 10.5 \mathrm{~J} \mathrm{~g}^{-1}$

LUCITE; solid; (polymethylmethacrylate); (E.I. duPont de Nemours and Company trade name); repeating unit $\mathrm{C}_{5} \mathrm{H}_{8} \mathrm{O}_{2}$.

gross heat of combustion: at $25^{\circ} \mathrm{C}$.

$q_{v}($ gross $)=6367.8 \pm 2.3 \mathrm{cal} \mathrm{g}^{-1}$
$q_{v}($ gross $)=26642.9 \pm 9.6 \mathrm{Jg}^{1}$

specific heat: assume values refer to room temperature.

$\begin{array}{ll}\operatorname{Cp}\left(\operatorname{cal} g^{-1} K^{-1}\right) & =0.343 \\ \operatorname{Cp}\left(J g^{-1} K^{-1}\right) & =1.435\end{array}$

2,5-METHYLOLMELAMINE, HARDENED; solid; elementary analysis; carbon, $34.19 \%$; hydroger, $5.27 \%$; oxygen, $17.07 \%$; nitrogen, $43.47 \%$; empirical formula $\mathrm{C}_{13} \mathrm{H}_{20} \mathrm{O}_{4} \mathrm{~N}_{12}$; a melanineformaldehyde polymer.

gross heat of combustion: at $25^{\circ} \mathrm{C}$.

$q_{v}($ gross $)=3861.6 \pm 1.3 \mathrm{cal} \mathrm{g}^{-1}$
$q_{v}($ gross $)=16156.9 \pm 5.4 \mathrm{~J} \mathrm{~g}^{1}$

2,5-METHYLOLMELAMINE, UNHARDENED; solid; elementary analysis; carbon, $32.70 \%$; hydrogen, $5.53 \%$; oxygen, $20.51 \%$; nitrogen, $41.26 \%$; empirical formula $\mathrm{C}_{5 .} \mathrm{H}_{31} \mathrm{O}_{2.5} \mathrm{~N}_{6}$; a nelamine-formaldehyde polymer.

gross heat of combustion: at $25^{\circ} \mathrm{C}$.

$q_{v}($ gross $)=3721.8 \pm 1.2 \mathrm{cal} \mathrm{g}^{-1}$
$q_{v}($ gross $)=15572.0 \pm 5.0 \mathrm{Jg}^{1}$ 


\section{POL YMERS}

NORYL RESIN III; solid; composition: polystyrene $=47 \%$; polyphenylene oxide $=47 \%$; polybutadiene $=5 \%$; and polyethylene $=1 \%$.

heat of fusion: between 83 and $103^{\circ} \mathrm{C}$.

$L_{f}=0.2 \mathrm{cal}^{-1}$
$L_{f}=0.8 \mathrm{~J} \mathrm{~g}^{-1}$

specific heat:

temperature, ${ }^{\circ} \mathrm{C}$

$$
\begin{gathered}
\left.C p(c a) g^{-1} K^{-1}\right) \\
0.178 \\
0.242 \\
0.228 \\
0.316 \\
0.393 \\
0.439 \\
0.483 \\
0.503
\end{gathered}
$$$$
\mathrm{Cp}\left(\mathrm{J} \mathrm{g}^{-1} \mathrm{~K}^{-1}\right)
$$$$
-79
$$$$
+4
$$$$
+25
$$$$
+50
$$$$
+100
$$$$
+151
$$$$
+209
$$$$
+246
$$

0.745

1.013

1.205

1.322

1.644

1.837

2.021

2.105

specific heat change at glass transition temperature:

$\begin{array}{ccc}\text { temperature, }{ }^{\circ} \mathrm{C} & \Delta C p\left(\operatorname{cal} \mathrm{g}^{-1} \mathrm{~K}^{-1}\right) & \Delta C p\left(\mathrm{~J} \mathrm{~g} \mathrm{~g}^{-1} \mathrm{~K}^{-1}\right) \\ -61 & 0.005 & 0.021 \\ +141 & 0.044 & 0.184\end{array}$


NYLON-6; solid; (poly-e-caprolactam); repeating unit: $\mathrm{C}_{6} \mathrm{H}_{11}$ NO.

gross heat of combustion: at $20^{\circ} \mathrm{C}$.

slow condensation

$\begin{array}{ll}q_{v}(\text { gross }) & =7575 \mathrm{cal} \mathrm{g}^{-1} \\ q_{v}(\text { net }) & =31694 \mathrm{~J} \mathrm{~g}^{1}\end{array}$

rapid condensation

$q_{v}($ gross $)=7587 \mathrm{cal} \mathrm{g}^{-1}$
$q_{v}($ gross $)=31744 \mathrm{~J} \mathrm{~g}^{-1}$

Miranid $\mathrm{H}: \quad 25^{\circ} \mathrm{C}$

$q_{v}($ gross $)=7454.1 \pm 2.8 \mathrm{cal} \mathrm{g}^{-1}$
$q_{v}($ gross $)=31188.0 \pm 11.7 \mathrm{~J} \mathrm{~g}^{-1}$

specific heat: $0-160^{\circ} \mathrm{C}$; amorphous Nylon-6.

$\begin{array}{ll}\operatorname{Cp}\left(\text { cal } \dot{g}^{-1} \mathrm{deg}^{-1}\right) & =-0.085+1.5 \times 10^{-3} \mathrm{~T} \\ \operatorname{Cp}\left(\mathrm{Jg}^{-1} \mathrm{deg}^{-1}\right) & =-0.356+6.3 \times 10^{-3} \mathrm{~T}\end{array}$

melting point: maximum melting temperature is $225^{\circ} \mathrm{C}(498 \mathrm{~K})$.

heat of fusion: at $225^{\circ} \mathrm{C}$.

$L_{f}=45 \mathrm{cal} \mathrm{g}^{-1}$
$L_{f}=188 \mathrm{~J} \mathrm{~g}^{-1}$

glass transition temperatures: depends upon prior treatment of polymer and amount of monomer present.

Physical Form

Flake

Undrawil filaments

Pre-melt annealed

Melt annealed
Transition

Temp. $\left({ }^{\circ} \mathrm{C}\right)$

45- 48

37

38

20
Percent

Monomer

Present

1.7
4
4
11

4

11 


\section{POL YMERS}

NYLON-6; 1iquid; (poly-e-caprolactam); repeating unit: $\mathrm{C}_{6} \mathrm{H}_{1}$ NO.

specific heat:

$160^{\circ}$ to $280^{\circ} \mathrm{C}$.

$\operatorname{Cp}\left(\operatorname{cal} g^{-1} K^{-1}\right)=0.485+3.36 \times 10^{-4}{ }^{\top}$
$\operatorname{Cp}\left(J g^{-1} K^{-1}\right)=2.029+1.406 \times 10^{-3} T$ 
NYLON-6,6; solid; (polyhexamethyleneadipamide); repeating unit: $\mathrm{C}_{12} \mathrm{H}_{22} \mathrm{~N}_{2} \mathrm{O}_{2}$; average nol. wt. is 11000 .

gross heat of combustion: at $20^{\circ} \mathrm{C}$.

$q_{v}($ gross $)=7581 \mathrm{cal} \mathrm{g}^{-1}$
$q_{v}($ gross $)=31719 \mathrm{Jg}^{1}$

[23]

U1tramid $A$

$q_{v}($ gross $)=7543.3+2.4 \mathrm{cal} \mathrm{g}^{-1}$
$q_{v}($ gross $)=31563.310 .0 \mathrm{~J} \mathrm{~g}^{-1}$

[60]

specific heat: $0{ }^{\circ} \mathrm{C}$ to $280{ }^{\circ} \mathrm{C}$.

\begin{tabular}{|c|c|c|}
\hline $\begin{array}{c}\text { Temperature, }{ }^{\circ} \mathrm{C} \\
\text { Annealed }\end{array}$ & $C p\left(\operatorname{cal} g^{-1} K^{-1}\right)$ & $C p\left(J g^{-1} K^{-1}\right)$ \\
\hline $\begin{array}{r}0 \\
20 \\
40 \\
60 \\
80 \\
100 \\
120 \\
140 \\
160 \\
180 \\
200 \\
220 \\
240 \\
250 \\
260 \\
270 \\
280\end{array}$ & $\begin{array}{l}0.311 \\
0.333 \\
0.367 \\
0.424 \\
0.468 \\
0.505 \\
0.548 \\
0.583 \\
0.642 \\
0.620 \\
0.646 \\
0.664 \\
0.80 \\
1.13 \\
2.75 \\
1.3 \\
0.75\end{array}$ & $\begin{array}{c}1.301 \\
1.393 \\
1.536 \\
1.774 \\
1.958 \\
2.113 \\
2.293 \\
2.439 \\
2.686 \\
2.594 \\
2.703 \\
2.778 \\
3.347 \\
4.728 \\
11.51 \\
5.439 \\
3.138\end{array}$ \\
\hline Drawli & & \\
\hline $\begin{array}{r}0 \\
20 \\
40 \\
60 \\
80 \\
100 \\
120 \\
140 \\
160 \\
130 \\
200 \\
220 \\
240 \\
250 \\
260 \\
270 \\
280\end{array}$ & $\begin{array}{l}0.302 \\
0.345 \\
0.376 \\
0.420 \\
0.458 \\
0.479 \\
0.503 \\
0.528 \\
0.542 \\
0.560 \\
0.582 \\
0.567 \\
0.676 \\
1.55 \\
6.5 \\
1.35 \\
0.75\end{array}$ & $\begin{array}{c}1.264 \\
1.443 \\
1.573 \\
1.757 \\
1.916 \\
2.004 \\
2.105 \\
2.209 \\
2.268 \\
2.343 \\
2.435 \\
2.372 \\
2.828 \\
6.485 \\
27.20 \\
5.648 \\
3.138\end{array}$ \\
\hline
\end{tabular}


melting point:

$\begin{array}{rccc}\text { Ribbon } & \begin{array}{c}\text { Undrawn } \\ \text { Filaments }\end{array} & \begin{array}{c}\text { Drawn } \\ \text { Filaments }\end{array} & \text { Annealed } \\ 263 & 264 & 259 & 262 \\ 25 & 40 & 18 & 30\end{array}$

Heat of fusion:

$\begin{array}{lrcc} & \text { Ribbon } & \text { Drawn Filaments } & \text { Annealed } \\ L_{f}\left(\mathrm{cal} \mathrm{g}^{-1}\right) & 29.0 & 29.5 & 33.3 \\ \mathrm{~L}_{f}\left(\mathrm{Jg}^{-1}\right) & 121.3 & 165.3 & 139.3\end{array}$

NYLON-6,10; solid; ribbon; (polyhexamethylenesebacamide); repeating unit: $\mathrm{C}_{16} \mathrm{H}_{30} \mathrm{~N}_{2} \mathrm{O}_{2}$; average mol. wt. 9250 .

specific heat: ribbon

Temperature, ${ }^{\circ} \mathrm{C}$

0
20
40
60
80
100
120
140
160
180
200
220
240
250
260
270
280

$$
C p\left(\operatorname{cal} g^{-1} K^{-1}\right)
$$

0.327

0.373

0.420

0.486

0.512

0.518

0.531

0.561

0.592

0.588

0.686

1.95

0.737

0.639

0.627

0.632

0.639

$$
\operatorname{Cp}\left(J g^{-1} k^{-1}\right)
$$

1.368

1.561

1.757

2.033

2.142

2.167

2.222

2.347

2.477

2.460

2.870

8.159

3.084

2.674

2.623

2.644

2.674

melting point:

M.P. $=225^{\circ} \mathrm{C}$; melting range $30^{\circ}-40^{\circ} \mathrm{C}$

heat of fusion: ribbon

$L_{f}\left(c_{f} g^{-1}\right)=26.3$
$L_{f}\left(J g^{-1}\right)=110.0$ 
PHENOL-FORMALDEHYDE RESIN, HARDENED (EDELKUNSTHARZ B); solid; empirical formula: $\mathrm{C}_{13} \mathrm{H}_{12} \mathrm{O}_{2}$.

gross heat of combustion: at $25^{\circ} \mathrm{C}$.

$q_{v}($ gross $)=7554.3 \pm 2.1 \mathrm{cal} \mathrm{g}^{-1}$
$q_{v}($ gross $)=31607.2 \pm 8.8 \mathrm{~J} \mathrm{~g}^{1}$

PHENOL-FORMALDEHYDE RESIN, UNHARDENED; solid; empirical formula $\mathrm{C}_{14} \mathrm{H}_{14} \mathrm{O}_{3}$.

gross heat of combustion: at $25^{\circ} \mathrm{C}$.

$q_{v}($ gross $)=7204.2 \pm 2.0 \mathrm{cal} \mathrm{g}^{-1}$
$q_{v}($ gross $)=30142.4 \pm 8.4 \mathrm{~J} \mathrm{~g}^{2}$

POLYACENAPHTHYLENE; solid; amorphous; repeating unit: $\mathrm{C}_{12} \mathrm{H}_{8}$; degree of polymerization $\sim 600$ units.

gross heat of combustion: at $25^{\circ} \mathrm{C}$.

$q_{v}($ gross $)=9388.0 \pm 2.0 \mathrm{cal} \mathrm{g}^{-1}$
$q_{v}($ gross $)=39279.4 \pm 8.4 \mathrm{~J} \mathrm{~g}^{-1}$

POLYALLYLPHTHALATE; solid; empirical formula $\mathrm{C}_{14} \mathrm{H}_{14} \mathrm{O}$.

gross heat of combustion: at $25^{\circ} \mathrm{C}$.

$q_{v}$ (gross) $=6629.8 \pm 2.1 \mathrm{cal} \mathrm{g}^{-1}$
$q_{v}($ gross $)=27739.1 \pm 8.8 \mathrm{~J} \mathrm{~g}^{1}$

POLYBUTADIENE $41{ }^{\circ} \mathrm{F}$; solid; Analysis: carbon, $88.47 \%$; hydrogen, $11.160 \%$; sulfur, $0.083 \%$; oxygen, $0.12 \%$; ash, $0.34 \%$; phenyl-B-naphthaylamine, $0.10 \%$; Repeating unit:

$\mathrm{C}_{4} \mathrm{H}_{6}$.

specific heat:

Temperature, $\mathrm{K}$

$$
\left.C p(c a) g^{-1} k^{-1}\right)
$$$$
\operatorname{Cp}\left(J \mathrm{~g}^{-1} \mathrm{~K}^{-1}\right)
$$

0.22416

200

0.359

0.6624

0.433

300

0.471

0.9379

1.50

1.81

0.4940

1.97

2.067 


\section{POLYMERS}

POLYBUTADIENE $122^{\circ} \mathrm{F}$; solid; Analys is: carbon, $88.66 \%$; hydrogen, $11.180 \%$; sulfur, $0.150 \%$; - oxygen, 0.145\%; ash, .097\%; phenyl-B-naphthylamine, $0.12 \%$; repeating unit:

$\mathrm{C}_{4} \mathrm{H}_{6}$.

specific heat:

Temperature, $k$

$$
\operatorname{cp}\left(\operatorname{cal} g^{-1} \mathrm{~K}^{-1}\right)
$$$$
C p\left(J g^{-1} k^{-1}\right)
$$

50
100
150
200
250
300
330

0.08640

0.15777

0.3615

50
100

0.22299

0.3967

0.4326

0.6601

200

0.4680

0.9330

250

0.4919

1.66

1.81

1.958

2.058

POLY-1-BUTENE; solid; isotactic; $34 \%$ crystallinity; repeating unit: $\mathrm{C}_{4} \mathrm{H}_{8}$; degree of polymerization $\sim 800$ units.

gross heat of combustion: at $25{ }^{\circ} \mathrm{C}$.

$q_{v}$ (gross) $=11109.4 \pm 0.5 \mathrm{cal}^{-\mathrm{g}^{-1}}$
$q_{v}$ (gross) $=46481.7 \pm 2.1 \mathrm{Jg}^{-1}$

POLY-iso-BUTENE; (Sample 1) viscous 1iquid; molecular weight 4900; (Sample 2) amorphous solid, rubberlike; molecular weight 200000; $0.033 \%$ ash; (Sample 3 ) solid;

Opanol B 100; repeating unit: $\mathrm{C}_{4} \mathrm{H}_{8}$.

gross heat of combustion: at $25^{\circ} \mathrm{C}$

Sample 1

$q_{v}$ (gross) $=11179.8 \pm 1.3 \mathrm{cal}^{-1}$
$q_{v}$ (gross) $=46776.3 \pm 5.4 \mathrm{~J} \mathrm{~g}^{-1}$

Sample 2

$q_{v}($ gross $)=11175.8 \pm 2.0 \mathrm{cal}^{-\mathrm{g}^{-1}}$
$q_{v}($ gross $)=46759.5 \pm 8.3 \mathrm{~J} \mathrm{~g}^{-1}$

Sample 3

$q_{v}($ gross $)=11179.1 \pm 4.3 \mathrm{cal} \mathrm{g}^{-1}$
$q_{v}($ gross $)=46773.4 \pm 18.0 \mathrm{~J} \mathrm{~g}^{1}$ 


\section{POL YMERS}

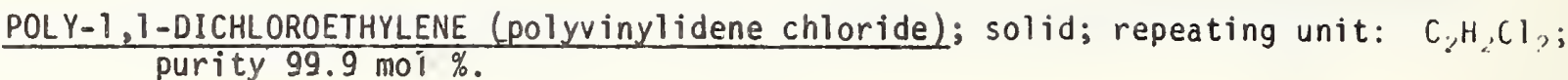

gross heat of combustion: at $25^{\circ} \mathrm{C}$.

$q_{v}($ gross $)=2515.1 \pm 2.1 \mathrm{cal} \mathrm{g}^{-1}$
$q_{v}($ gross $)=10523.2 \pm 8.8 \mathrm{Jg}^{-1}$

[31]

POLY-1,1-DIFLUOROETHYLENE (polyvinylidene fluoride); solid; repeating unit: $\mathrm{C}_{2} \mathrm{H}_{2} \mathrm{~F}_{2}$; particle size 100 mesh.

gross heat of combustion: at $25^{\circ} \mathrm{C}$.

$q_{v}($ gross $)=3529.1 \pm 0.4 \mathrm{cal} \mathrm{g}^{-1}$
$q_{v}($ gross $)=14765.8 \pm 1.7 \mathrm{~J} \mathrm{~g}^{1}$

[32]

POLY-2,6-DIMETHYLPHENYLENE ETHER; solid; repeating unit: $\mathrm{C}_{8} \mathrm{H}_{8} \mathrm{O} ; 20 \%$ crystallinity; degree of polymerization $\sim 300$ units.

gross heat of combustion: at $25^{\circ} \mathrm{C}$.

$q_{v}($ gross $)=8273.2 \pm 1.4 \mathrm{cal} \mathrm{g}^{-1}$
$q_{v}($ gross $)=34615.1 \pm 5.9 \mathrm{Jg}^{-1}$

POLY-4, 4'-DIOXYOIPHENYL-2,2-PROPANE CARBONATE; solid; empirical formula $\mathrm{C}_{16} \mathrm{H}_{14} \mathrm{O}_{3}$.

gross heat of combustion: at $25^{\circ} \mathrm{C}$.

PKU T 22

$q_{v}($ gross $)=7408.7 \pm 2.1 \mathrm{cal} \mathrm{g}^{-1}$
$q_{v}($ gross $)=30998.0 \pm 8.8 \mathrm{~J} \mathrm{~g}^{1}$

[60]

Lexan; $20 \%$ crystallinity; degree of polymerization $\sim 150$ units.

$q_{v}($ gross $)=7392.9 \pm 1.4 \mathrm{cal} \mathrm{g}^{-1}$
$q_{v}($ gross $)=30931.9 \pm 5.9 \mathrm{~J} \mathrm{~g}^{1}$

Makrolon; solid.

$q_{v}($ gross $)=7408.7 \pm 2.0 \mathrm{cal} \mathrm{g}^{-1}$
$q_{v}($ gross $)=30998.0 \pm 8.4 \mathrm{~J} \mathrm{~g}^{1}$ 
POLY- $\varepsilon$-ENANTHOLACTAN; solid; repeating unit: $\mathrm{C}_{7} \mathrm{H}_{1}{ }^{\mathrm{NO}}$.

gross heat of combustion: at $20^{\circ} \mathrm{C}$.

$q_{v}($ gross $)=7977.2 \pm 5.5 \mathrm{cal} \mathrm{g}^{-1}$
$q_{v}($ gross $)=33376.6 \pm 23.0 \mathrm{~J} \mathrm{~g}^{-1}$

POLYETHYLENE; solid; NBS Standard Reference Material (SRM) 1475; linear polymer; wt.-ave. molecular weight is 52000 ; methyl group content is 0.15 methyl groups per 100 carbon atoms; ash content 0.002 percent; 111 ppm of anti-oxidant [tetrakis-(methylene-3-( $3^{\prime}, 5^{\prime}$-di-tert-butyl-4'-hydroxpheny 1$)$ propionate) methane] added to polymer by manufacturer (E.I. Du Pont de Nemours and Co.); density at $23{ }^{\circ} \mathrm{C}$ is $0.9784 \mathrm{~g} \mathrm{~cm}^{-3}$; degree of crystallinity detd. to be 72 percent; repeating unit: $\mathrm{C}_{2} \mathrm{H}_{4}$.

gross heat of combustion: at $25^{\circ} \mathrm{C}$.

SRM 1475, crystallinity $72 \%$

$q_{v}($ gross $)=11073.7 \pm 2.1 \mathrm{cal}^{-g^{-1}}$
$q_{v}($ gross $)=46332.4 \pm 8.8 \mathrm{Jg}^{-1}$

SRM 1475 modified by recrystallization under high pressure; crystallinity $96 \%$.

$q_{v}($ gross $)=11058.6 \pm 2.0 \mathrm{cal}^{-\mathrm{g}^{-1}}$
$q_{v}($ gross $)=46269.1 \pm 8.1 \mathrm{~J} \mathrm{~g}^{-1}$

specific heat: SRM 1475

Temperature, $K$

10

100

200

250

273.15

298.15

320

360
$\left.C_{p}(c a) g^{-1} k^{-1}\right)$

0.0029

0.1632

0.2839

0.3537

0.3936

0.4376

0.4790

0.5904

$C p\left(J g^{-1} K^{-1}\right)$
0.0123
0.6829
1.1877
1.4800
1.6468
1.8308
2.0040
2.4702

0.0123

0.6829

1.6468

2.0040

2.4702 
POLYETHYLENE; solid; (sample 1) high pressure; molecular weight 18000-20000; crystallinity 50\%; (sample 2) linear; Marlex; crystallinity 98\%; (sample 3) low density; (sample 4) high density, Mirathen $\mathrm{H}$; repeating unit: $\mathrm{C}_{2} \mathrm{H}_{4}$.

gross heat of combustion: at $25^{\circ} \mathrm{C}$.

sample 1

$q_{v}($ gross $)=11093.6 \pm 0.8 \mathrm{cal}^{-\mathrm{g}^{-1}}$
$q_{v}($ gross $)=46415.6 \pm 3.3 \mathrm{~J} \mathrm{~g}^{-1}$

sample 2

$q_{v}($ gross $)=11080.9 \pm 2.0 \mathrm{cal}^{-1}$
$q_{v}($ gross $)=46362.5 \pm 8.4 \mathrm{~J} \mathrm{~g}^{-1}$

sample 3

$q_{v}($ gross $)=11042.7 \pm 3.5 \mathrm{cal} \mathrm{g}^{-1}$
$q_{v}($ gross $)=46202.7 \pm 14.6 \mathrm{~J} \mathrm{~g}^{2}$

[60]

\section{sample $4^{\circ}$}

$q_{v}($ gross $)=11102.9 \pm 4.6 \mathrm{cal} \mathrm{g}^{-1}$
$q_{v}($ gross $)=46485.5 \pm 19.2 \mathrm{~J} \mathrm{~g}^{-1}$

POLY- - -METHYL STYRENE; solid; anionic; amorphous; samples with different degrees of polymerization, 234000 unfts and 273000 units gave the same heat of combustion; repeating unit: $\mathrm{C}_{9} \mathrm{H}_{10}$.

gross heat of combustion: at $25^{\circ} \mathrm{C}$.

$q_{v}$ (gross) $=10111.8 \pm 2.8 \mathrm{cal} \mathrm{g}^{-1}$
$q_{v}($ gross $)=42307.8 \pm 11.7 \mathrm{~J} \mathrm{~g}^{1}$ 


\section{POL YMERS}

POLYPROPYLENE; solid; repeating unit: $\mathrm{C}_{3} \mathrm{H}_{6}$.

gross heat of combustion: at $25{ }^{\circ} \mathrm{C}$.

isotactic; $58 \%$ crystallinity

$q_{v}($ gross $)=11082.4 \pm 2.2 \mathrm{cal} \mathrm{g}^{-1}$
$q_{v}($ gross $)=46368.8 \pm 9.2 \mathrm{j} \mathrm{g}^{-1}$

degree of polymerization $~ 4000$ units

$q_{v}($ gross $)=11080.5 \pm 2.2 \mathrm{cal}^{-\mathrm{g}^{-1}}$
$q_{v}($ gross $)=46360.8 \pm 9.2 \mathrm{Jg}^{-1}$

Polypropylene VK166, untangled

$q_{v}($ gross $)=11021.2 \pm 5.1 \mathrm{cal} \mathrm{g}^{-1}$
$q_{v}($ gross $)=46112.7 \pm 21.3 \mathrm{Jg}^{1}$

POLYSTYRENE: solid; repeating unit: $\mathrm{C}_{8} \mathrm{H}_{8}$.

gross heat of combustion: at $25^{\circ} \mathrm{C}$.

isotactic, $40 \%$ crystallinity

$q_{v}$ (gross) $=9909.9 \pm 3.1 \mathrm{cal} \mathrm{g}^{-1}$
$q_{v}($ gross $)=41463.0 \pm 13.0 \mathrm{~J} \mathrm{~g}^{-1}$

isotactic, amorphous

$q_{v}($ gross $)=9918.5 \pm 2.4 \mathrm{cal} \mathrm{g}^{-1}$
$q_{v}($ gross $)=41499.0 \pm 10.0 \mathrm{~J} \mathrm{~g}^{-1}$

atactic, amorphous

$q_{v}($ gross $)=9921.2 \pm 1.4 \mathrm{cal} \mathrm{g}^{-1}$
$q_{v}($ gross $)=47510.3 \pm 5.9 \mathrm{~J} \mathrm{~g}^{1}$

polystyrol BW

$q_{v}($ gross $)=9922.4 \pm 4.0 \mathrm{cal} \mathrm{g} g^{-1}$
$q_{v}($ gross $)=41515.3 \pm 16.7 \mathrm{~J} \mathrm{~g}^{-1}$

polystyrol P70

$q_{v}$ (gross) $=9900.5 \pm 3.0 \mathrm{cal} \mathrm{g}^{-1}$
$q_{v}($ gross $)=41423.7 \pm 12.6 \mathrm{~J} \mathrm{~g}^{-1}$ 
POLYUREA (POLYHARNSTOFF); solid; empirical formula is $\mathrm{C}_{15} \mathrm{H}_{18} \mathrm{O}_{4} \mathrm{~N}_{4}$; a polymer of adipic acid diamide plus $(2,4-2,6)$-toluene diisocyanate.

gross heat of combustion: at $25^{\circ} \mathrm{C}$.

$q_{v}($ gross $)=5954.0 \pm 1.7 \mathrm{cal} \mathrm{g}^{-1}$
$q_{v}($ gross $)=24911.5 \pm 7.1 \mathrm{~J} \mathrm{~g}^{1}$

RESIN, ABS I; solid; copolymer of styrene-acrylonitrile $=76 \%$; and polybutadiene $=15 \%$.

specific heat:

temperature $\left({ }^{\circ} \mathrm{C}\right)$

$$
\left.\operatorname{cp}(c a) g^{-1} K^{-1}\right) \quad \operatorname{cp}\left(J g^{-1} K^{-1}\right)
$$

$$
\begin{aligned}
& -110 \\
& -56 \\
& +4 \\
& +25 \\
& +55 \\
& +107 \\
& +157 \\
& +204
\end{aligned}
$$$$
0.184
$$$$
0.240
$$$$
0.770
$$$$
0.311
$$$$
1.004
$$

0.338

0.373

0.518

specific heat change at glass transition temperature:

$\begin{array}{ccc}\text { temperature }\left({ }^{\circ} \mathrm{C}\right) & \Delta C p\left(\mathrm{Cal} \mathrm{g}^{-1} \mathrm{~K}^{-1}\right) & \Delta \mathrm{Cp}\left(\mathrm{J} \mathrm{g}^{-1} \mathrm{~K}^{-1}\right) \\ -85 & 0.014 & 0.059 \\ +103 & 0.069 & 0.289\end{array}$

RILSAN; solid; (poly-11-aminodecanoic acid); empirical formula is $\mathrm{C}_{11} \mathrm{H}_{21} \mathrm{ON}$.

gross heat of combustion: at $25^{\circ} \mathrm{C}$.

$q_{v}$ (gross) $=8840.3 \pm 2.2 \mathrm{cal} \mathrm{g}^{-1}$
$q_{v}$ (gross) $=36987.8 \pm 8.4 \mathrm{~J} \mathrm{~g}^{1}$ 
TEFLON; solid; (polytetrafluoroethylene); repeating unit: $C_{4} F_{4}$; density, $2.2 \mathrm{~g} \mathrm{~cm}^{-3}$.

gross heat of combustion in oxygen: at $25{ }^{\circ} \mathrm{C}$; combustion products are gaseous carbon dioxide and carbon tetraftuoride.

$q_{v}$ (gross) $=1196.7 \pm 10.0 \mathrm{ca}^{3} \mathrm{~g}^{-1}$
$q_{v}$ (gross) $=5007.0 \pm 42 \mathrm{Jg}^{-1}$

gross heat of combustion in fluorine: $25^{\circ} \mathrm{C}$; combustion product is carbon tetrafluoride.

$q_{v}($ gross $)=2478.8 \pm 0.7 \mathrm{cal} \mathrm{g}^{-1}$
$q_{v}($ gross $)=10371.3 \pm 3.1 \mathrm{~J} \mathrm{~g}^{1}$

specific heat:

Temperature, $k$

50
100
150
200
250
270
280
310
330
360

$C p\left(c a 1 g^{-1} K^{-1}\right)$
.04821
.09218
.13380
.17706
.21076
.22768
.24187
.24402
.24809
.25669

$\operatorname{Cp}\left(\operatorname{cal} \mathrm{g}^{-1} \mathrm{~K}^{-1}\right)$

.04821

.09218

.17706

.21076

.22768

24402

.25669
$C p\left(J g^{-1} K^{-1}\right)$

.2017

.3857

.5598

.7408

.8818

.9526

1.012

1.021

1.038

1.074

[106]

transition temperatures:

$293 \mathrm{~K}\left(20^{\circ} \mathrm{C}\right)$

$303 \mathrm{~K}\left(30^{\circ} \mathrm{C}\right)$

[106]

heat of transition: for the temperature range 280 to $310 \mathrm{~K}$.

$L_{f}=8.72 \mathrm{cal}^{-g^{-1}}$
$L_{f}=36.5 \mathrm{~J} \mathrm{~g}^{-1}$

TROCKENLEIM; solid; el ementary analysis; carbon, $35.49 \%$; hydrogen, $6.19 \%$; oxygen, $30.91 \%$; nitrogen, $27.47 \%$; a urea-formaldehyde resin: empirical formula $\mathrm{C}_{3} \mathrm{H}_{6} \mathrm{O}_{2} \mathrm{~N}_{2}$.

gross heat of combustion: at $25{ }^{\circ} \mathrm{C}$.

$q_{v}$ (gross) $=3800.4 \pm 2.0 \mathrm{cal} \mathrm{g}^{-1}$
$q_{v}($ gross $)=15900.9 \pm 8.4 \mathrm{Jg}^{-1}$ 
POLYPEPTIDE, PHENYLALANINE; solid; repeating unit: $\mathrm{C}_{g} \mathrm{H}_{9} N O$.

gross heat of combustion: at $25^{\circ} \mathrm{C}$.

benzene soluble

$q_{v}($ gross $)=7503.2 \pm 6.0 \mathrm{cal} \mathrm{g}^{-1}$
$q_{v}($ gross $)=31393.4 \pm 25.1 \mathrm{~J} \mathrm{~g}^{-1}$

benzene insoluble

$q_{v}($ gross $)=7479.1 \pm 6.9 \mathrm{cal} \mathrm{g}^{-1}$
$q_{v}($ gross $)=31292.6 \pm 28.9 \mathrm{~J} \mathrm{~g}^{-1}$

POLYPEPTIDE, POLY-N-ETHYL-N-METHYL PROPIONAMIDE; solid; density $1.4400 \mathrm{~g} \mathrm{~cm}^{-3}$; repeating unit: $\mathrm{C}_{6} \mathrm{H}_{13} \mathrm{NO}$.

gross heat of combustion: at $25{ }^{\circ} \mathrm{C}$.

$q_{v}($ gross $)=8069.3 \pm 1.0 \mathrm{cal} \mathrm{g}^{-1}$
$q_{v}($ gross $)=33762.0 \pm 4.2 \mathrm{~J} \mathrm{~g}^{1}$

POLYPETIDE, SARCOSINE; solid; repeating unit: $\mathrm{C}_{3} \mathrm{H}_{5} \mathrm{NO}$.

gross heat of combustion: at $25{ }^{\circ} \mathrm{C}$.

$q_{v}($ gross $)=5626.4 \pm 4.2 \mathrm{cal} \mathrm{g}^{-1}$
$q_{v}($ gross $)=23540.9 \pm 17.6 \mathrm{~J} \mathrm{~g}^{-1}$

POLYSACCHARIDES

CELLULOSE; solid; repeating unit: $\mathrm{C}_{6} \mathrm{H}_{10} \mathrm{O}_{5}$, constitutes about 60 percent of the composition of wood; hydrolysis can reduce cellulose to a cellobiose repeating unit, $\mathrm{C}_{12} \mathrm{H}_{22} \mathrm{O}_{11}$, and ultimately to glucose, $\mathrm{C}_{6} \mathrm{H}_{12} \mathrm{O}_{6}$.

gross heat of combustion: at $30^{\circ} \mathrm{C}$.

from cotton linters:

$q_{v}($ gross $)=4165.0 \mathrm{cal} \mathrm{g}^{-1}$
$q_{v}($ gross $)=17426.4 \mathrm{~J} \mathrm{~g}^{1}$

from wood pulp:

$q_{v}($ gross $)=4172.0 \mathrm{cal} \mathrm{g}^{-1}$
$q_{v}($ gross $)=17455.6 \mathrm{~J} \mathrm{~g}^{2}$ 


\section{POL YSACCHARIDES}

a-CELLULOSE, FROM LOBLOLLY PINE WOOD (Pinus taeda L.): solid; modification of cellulose which has the highest degree of polymerization; chief constituent of paper pulp: $46.70 \% \alpha$-cellulose [81]; repeating unit: $\mathrm{C}_{6} \mathrm{H}_{3} \mathrm{O}_{5}$.

specific heat: $60-140{ }^{\circ} \mathrm{C}$; samples were oven-dried at $100^{\circ} \mathrm{C}$ for 12 hours before measurements were made.

$$
\begin{array}{ll}
\operatorname{Cp}\left(c_{1} g^{-1} K^{-1}\right) & =-0.01708+9.9 \times 10^{-4}{ }^{\top} \\
C p\left(J g^{-1}\right) & =-0.07146+4.14 \times 10^{-3} T
\end{array}
$$

CELLULOSE, HOLOCELLULOSE, FROM LOBLOLLY PINE WOOD; (Pinus taeda L.); solid; total waterinsoluble carbohydrate constituents of 10blolly pine wood; $71.18 \%$ holocellulose [81]; repeating unit: $\mathrm{C}_{6} \mathrm{H}_{10} \mathrm{O}_{5}$.

specific heat: $60-140^{\circ} \mathrm{C}$; samples were oven-dried at $100{ }^{\circ} \mathrm{C}$ for 12 hours before measurements were made.

$$
\begin{aligned}
& C p\left(\operatorname{cal} g^{-1} K^{-1}\right)=-0.01724+9.9 \times 10^{-4} T^{T} \\
& C p\left(J g^{-1} K^{-1}\right)=-0.07213+4.14 \times 10^{-3} T
\end{aligned}
$$

CELLULOSE DIACETATE; solid; repeating unit: $\mathrm{C}_{10} \mathrm{H}_{10} 0_{7}$; density $=1.36 \mathrm{~g} \mathrm{~cm}^{-3}$ at $20{ }^{\circ} \mathrm{C} ; 0.04 \%$ ash.

gross heat of combustion: assume values refer to room temperature.

$q_{v}($ gross $)=4446.2 \mathrm{cal} \mathrm{g}^{-1}$
$q_{v}($ gross $)=18602.9 \mathrm{~J} \mathrm{~g}^{1}$

CELLULOSE TRIACETATE; solid; repeating unit: $\mathrm{C}_{12} \mathrm{H}_{16} \mathrm{O}_{8}$.

gross heat of combustion: assume values refer to room temperature.

$q_{y}$ (gross) $=4511.5 \mathrm{cal}^{-1}$
$q_{y}($ gross $)=18876.1 \mathrm{Jg}^{-1}$

COTTON (THREAD); solid; staple fibers surrounding the seeds of various species of Gossypium; absorbent cotton is almost pure a-cellulose: $\mathrm{CH}_{1.774} 0_{0.887}$ is elemental analysis of thread used in combustion measurements.

gross heat of combustion: at $25{ }^{\circ} \mathrm{C}$.

$q_{v}($ gross $)=4050 \mathrm{cal} \mathrm{g}^{-1}$
$q_{v}($ gross $)=16945 \mathrm{~J} \mathrm{~g}^{1}$

$q_{v}($ gross $)=4108 \mathrm{cal} \mathrm{g}^{-1}$
$q_{v}($ gross $)=17188 \mathrm{Jg}^{-1}$ 


\section{POLYSACCHARIDES}

DEXTRIN; solid; general formula; $\mathrm{C}_{6} \mathrm{H}_{1} \mathrm{O}_{5}$; partial hydrolysis product of starch containing a variable number of glucose units; dextrin is obtained in several different grades by heating starch for varying lengths of time over the temperature range from $170^{\circ}$ to $240^{\circ} \mathrm{C}$.

gross heat of combustion: assume values refer to room temperature.

$q_{v}($ gross $)=4110 \mathrm{cal} \mathrm{g}^{-1}$
$q_{v}($ gross $)=17196 \mathrm{~J} \mathrm{~g}^{1}$

GLYCOGEN; solid; generai formula; $\mathrm{C}_{6} \mathrm{H}_{10} \mathrm{O}_{5}$; polysaccharide found in the liver and muscles of man and animals; can be hydrolyzed to glucose.

gross heat of combustion: assume values refer to room temperature.

$q_{v}($ gross $)=4188 \mathrm{cal} \mathrm{g}^{-1}$
$q_{v}($ gross $)=17523 \mathrm{~J} \mathrm{~g}^{1}$

INULIN; solid; repeating unit: $\mathrm{C}_{6} \mathrm{H}_{10} \mathrm{O}_{5}$; similar in physical appearance to starch except upon complete hydrolysis yields fructose instead of glucose; inulin is the chief source of fructose.

gross heat of combustion: assume values refer to room temperature.

$q_{v}$ (gross) $=4190 \mathrm{cal} \mathrm{g}^{-1}$
$q_{v}($ gross $)=17531 \mathrm{~J} \mathrm{~g}^{1}$

INULIN TRIACETATE; solid; repeating unit: $\mathrm{C}_{12} \mathrm{H}_{16} \mathrm{O}_{8}$; dried at $120{ }^{\circ} \mathrm{C}$ in vacuum over $\mathrm{P}_{2} \mathrm{O}_{5}$.

gross heat of combustion: assume values refer to room temperature.

$q_{v}($ gross $)=4522 \mathrm{cal} \mathrm{g}^{-1}$
$q_{v}($ gross $)=18920 \mathrm{~J} \mathrm{~g}^{1}$

STARCH; solid; general formula; $\mathrm{C}_{6} \mathrm{H}_{10} \mathrm{O}_{5}$; polysaccharide is widely distributed in the vegetable kingdom and is stored in all grains and tubers.

gross heat of combustion: assume values refer to room temperature.

$q_{v}($ gross $)=4200 \mathrm{cal} \mathrm{g}^{-1}$
$q_{v}($ gross $)=17570 \mathrm{~J} \mathrm{~g}^{-1}$ 


\section{POLYSACCHARIDES}

STARCH TRIACETATE; solid; repeating unit: $\mathrm{C}_{12} \mathrm{H}_{16} \mathrm{O}_{8}$.

gross heat of combustion: assume values refer to room temperature.

$q_{v}$ (gross) $=4499 \mathrm{cal} \mathrm{g}^{-1}$
$q_{v}$ (gross) $=18824 \mathrm{~J} \mathrm{~g}^{2}$

[111]

XYLAN; solid; repeating unit; $\mathrm{C}_{5} \mathrm{H}_{8} \mathrm{O}_{4}$; polysaccharide which often occurs in association with cellulose; xylose is the repeating unit; occurs in large amounts (20-40\%) in cereal straws and brans.

gross heat of combustion: assume values refer to room temperature.

$q_{v}($ gross $)=4242.8 \mathrm{cal} \mathrm{g}^{-1}$
$q_{v}($ gross $)=17751.9 \mathrm{Jg}^{1}$

PORCELAIN; solid; a mixture of clays, quartz, and feldspar, usually containing at least $25 \%$ aluminum. Sample 1: $\mathrm{SiO}_{2}, 65.14 \% ; \mathrm{Al}_{2} \mathrm{O}_{3}, 28.76 \% ; \mathrm{Fe}_{2} \mathrm{O}_{3}, 1.08 \% ; \mathrm{K}_{2} 0,3.61 \%$; $\mathrm{MgO}, 1.21 \%$; sample 2: $\mathrm{SiO}_{2}, 41.1 \% ; \mathrm{Al}_{2} \mathrm{O}_{3}, 57.1 \% ; \mathrm{Fe}_{2} \mathrm{O}_{3}, 0.3 \% ; \mathrm{MgO}, 0.5 \% ; \mathrm{CaO}$, traces.

specific heat: temperature range $0-1600{ }^{\circ} \mathrm{C}$.

sample 1

$\operatorname{Cp}\left(\right.$ cal $\left._{-1} g^{-1} K^{-1}\right)=0.10848+2.6497 \times 10^{-4} T-7.7124 \times 10^{-8} T^{2}$
$\operatorname{Cp}\left(\mathrm{J} \mathrm{g}^{-1}\right)=0.45388+1.10863 \times 10^{-3} T=3.2269 \times 10^{-7} T^{2}$

sample 2

$C p\left(\right.$ cal $\left.g^{-1} K^{-1}\right)=0.20698+1.1542 \times 10^{-4} T-5.685 \times 10^{-9} T^{2}$

$\mathrm{Cp}\left(\mathrm{J} \mathrm{g} \mathrm{g}^{-1} \mathrm{~K}^{-1}\right)=0.86600+4.8292 \times 10^{-4} \mathrm{~T}-3.2319 \times 10^{-8} \mathrm{~T}^{2}$

[118]

PROPELLANT, OS/AP; solid; oxygen-styrene copolymer was prepared by passing oxygen at 1 atm through styrene for 24 hours in the presence of azobisisobutyronitrile; the reaction was completed by adding benzoyl peroxide and maintaining the solution at $50^{\circ} \mathrm{C}$ for another 24 hours until it became sufficiently viscous; (OS/AP) = (oxygen-styrene copolymer) - ammonium perchlorate composite propellant.

gross heat of combustion: assume values refer to room temperature.

copolymer (wt. \%)

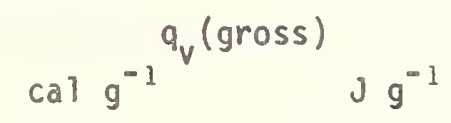

30

25

20

$\begin{array}{ll}1109 \pm 10 & 4640 \pm 42 \\ 1168 \pm 23 & 4887 \pm 96 \\ 1301 \pm 8 & 5443 \pm 33\end{array}$


PROPELLANT, PS/AP; solid; inhibitor-free styrene was polymerized by benzoyl peroxide and mixed with ammonium perchlorate; PS/AP = polystyrene-ammonium perchlorate composite propellant.

gross heat of combustion: assume values refer to room temperature.

polystyrene (Wt. \%)

30
25
20

$$
\begin{array}{rl}
\text { cal } \mathrm{g}^{-1} & \mathrm{~J} \mathrm{~g}_{v} \text { (gross) } \\
1017 \pm 15 & 4255 \pm 63 \\
1101 \pm 10 & 4607 \pm 42 \\
1291 \pm 5 & 5402 \pm 21
\end{array}
$$

PROPELLANT X14; solid; double base propellant processed by water mixing nitrocellulose with nitrogiycerine and a stabilizer (such as: $N, N^{\prime}-$ diethyl-N, $N^{\prime}-$ diphenyl urea, or 2-nitrodiphenylamine).

specific heat: 283 to $343 \mathrm{~K}$.

$$
\begin{array}{ll}
\left.C p(c a) g^{-1} K^{-1}\right) & =0.118+6.60 \times 10^{-4} T \\
C p\left(J g^{-1} K^{-1}\right) & =0.494+2.761 \times 10^{-3} T
\end{array}
$$

\section{PROTEIN}

BOVINE CHYMOTRYPSINOGEN A; solid; the anhydrous material is a protein from beef pancreas made up of 245 amino acids with molecular weight of 25646 .

specific heat:

$\begin{array}{ccc}\text { temperature }(K) & \left.C p(c a) g^{-1} K^{-1}\right) & C p\left(J g^{-1} K^{-1}\right) \\ 10 & 0.00448 & 0.01874 \\ 100 & 0.1120 & 0.4686 \\ 150 & 0.1696 & 0.7096 \\ 200 & 0.2153 & 0.9008 \\ 250 & 0.2617 & 1.9050 \\ 298.15 & 0.3090 & 1.2929 \\ 300 & 0.3109 & 1.3008\end{array}$

CASEIN, MILK; solid; carbon $53 \%$; hydrogen $7 \%$; oxygen $22.65 \%$; nitrogen $15.7 \%$; sulfur $0.8 \%$; phosphorus $0.85 \%$.

gross heat of combustion: assume values refer to room temperature.

$q_{v}($ gross $)=5670 \pm 200 \mathrm{cal} \mathrm{g}^{-1}$
$q_{v}($ gross $)=23700 \pm 800 \mathrm{Jg}^{-1}$ 
COLLAGEN; solid; fibrous protein which comprises the major portion of the white fiber in connective tissues of the animal body; chief amino acids present are: proline, hydroxyproline, and glycine; converted to gelatine by boiling with water.

specific heat: $25^{\circ} \mathrm{C}$.

$\begin{array}{ccc}\text { moisfure content (wt. \%) } & C p\left(c a l g^{-1} K^{-1}\right) & C p\left(J g^{-1} K^{-1}\right) \\ 0 & 0.3723 & 1.5577 \\ 1.02 & 0.3490 & 1.4602 \\ 1.96 & 0.3659 & 1.5309 \\ 4.56 & 0.4014 & 1.6795 \\ 6.49 & 0.4061 & 1.6991 \\ 17.03 & 0.4280 & 1.7908\end{array}$

GELATINE; solid; a protein obtained from collagen by boiling skin, ligaments, tendons, bones etc. with water; can absorb 5 to 10 times its weight of water.

gross heat of combustion: assume values refer to room temperature.

As received: ash content, $0.6 \%-0.8 \%$; water content not determined.

$q_{v}$ (gross) $=4400 \mathrm{cal} \mathrm{g}_{1}^{-1}$

$q_{v}($ gross $)=18410 \mathrm{Jg}$

Ash free and moisture free

$q_{v}($ gross $)=5150 \pm 100 \mathrm{cal} \mathrm{g}^{-1}$
$q_{v}($ gross $)=21550 \pm 400 \mathrm{~J} \mathrm{~g}^{2}$ 
GLIADIN; solid; also called prolomin; simple vegetable protein found in gluten; typical composition: carbon, $52.7 \%$; hydrogen, $6.9 \%$; oxygen, $21.7 \%$; nitrogen, $17.7 \%$; sulfur, $1.0 \%$.

gross heat of combustion:

variation with thermal denaturation temperature.

denaturation

temp., ${ }^{\circ} \mathrm{C}$

cal $\mathrm{g}^{-q_{v} \text { (gross) }} \mathrm{J} \mathrm{g}^{-1}$

$\begin{array}{rll}30 & 5986.3 & 25046.7 \\ 35 & 5911.3 & 24732.9 \\ 50 & 5777.2 & 24171.8 \\ 70 & 5809.3 & 24306.1 \\ 110 & 5852.8 & 24488.1\end{array}$

[91]

variation with concentration of aqueous alcohol used in extraction.

\begin{tabular}{ccc} 
arcohot\% & \multicolumn{2}{c}{$\mathrm{cal} \mathrm{g}_{v}$ (gross) } \\
& & $\mathrm{J} \mathrm{g}^{-1}$ \\
40 & 5891 & 24648 \\
50 & 5869 & 24556 \\
60 & 5942 & 24861 \\
70 & 6028 & 25221
\end{tabular}

GLUTEN; solid; mixture of proteins found in the seeds of cereal grains (wheat, corn (maize), etc.) which remains after washing the starch out of flour with water; amino acids of glutens are: glutamic acid, leucine, proline, and arginine.

gross heat of combustion: variation with thermal denaturation temperature. denaturation temp., ${ }^{\circ} \mathrm{C}$ cal $g^{-1} \mathrm{~J} \mathrm{~g}^{-1}$

$\begin{array}{rll}30 & 5797.9 & 24258.4 \\ 35 & 5873.2 & 24573.5 \\ 50 & 5648.8 & 23634.6 \\ 70 & 5721.1 & 23937.1 \\ 110 & 5779.6 & 24181.8\end{array}$




\section{PROTEIN}

GLUTENIN; solid; wheat protein soluble in dilute alkalies.

gross heat of combustion: variation with thermal denaturation temperature.

\begin{tabular}{|c|c|c|}
\hline denaturation & & s) \\
\hline $\operatorname{temp} .,{ }^{\circ} \mathrm{C}$ & $\operatorname{cal} g^{-1}$ & $\mathrm{~J} \mathrm{~g}^{-1}$ \\
\hline $\begin{array}{r}30 \\
35 \\
50 \\
70 \\
110\end{array}$ & $\begin{array}{l}5710.1 \\
5730.3 \\
5583.1 \\
5654.0 \\
5699.9\end{array}$ & $\begin{array}{l}23891.1 \\
23975.6 \\
23359.7 \\
23656.3 \\
23848.4\end{array}$ \\
\hline
\end{tabular}

VITELLIN; solid; globutin protein from egg yolk; typical composition; water $49.4 \%$; protein $16.3 \%$; fat $31.9 \%$; carbohydrate $0.7 \%$; remainder includes small amounts of elements such as: phosphorus, sulfur, iron, and caicium.

gross heat of combustion: assume values refer to room temperature.

$q_{v}($ gross $)=5760 \mathrm{cal} \mathrm{g}_{1}^{-1}$
$q_{v}($ gross $)=24100 \mathrm{Jg}^{1}$

WOOL PROTEIN; solid; material is a keratin containing $14.3 \%$ of cystine, $3.9 \%$ of sulfur, and $16.85 \%$ nitrogen.

gross heat of combustion: assume values refer to room temperature and are corrected for moisture and ash content.

$q_{v}$ (gross) $=5609 \pm 2.3 \mathrm{cal} \mathrm{g}^{-1}$
$q_{v}($ gross $)=23468 \pm 9.6 \mathrm{~J} \mathrm{~g}^{-1}$ 
REFUSE, MUNICIPAL; solid; waste from the city of St. Louis; particle size of 1.5 inches and less, magnetic metal removed; (batch 1); from 210 samples collected April 1972 through February 1973, unclassified material; with only magnetic metal removed; moisture, $9.3-50.0 \%$; sulfur, $0.01-0.40 \%$; chlorine, $0.13-0.95 \%$; ash, 12.1-52.2\%. (batch 2); from 99 samples collected November 1973 through January 197.4, classified material; heavy fraction of waste removed by air-density separation; light fraction only; moisture, $11.1-66.3 \%$; sulfur, $0.05-0.28 \%$; chlorine, 0.14-0.94\%; ash, 7.6-21.4\%.

gross heat of combustion: assume values refer to room temperature.

(batch 1)

minimum $q_{v}($ gross $)=1683 \mathrm{cal} \mathrm{g}^{-1}$
minimum $q_{v}($ gross $)=7041 \mathrm{~J} \mathrm{~g}^{-1}$
maximum $q_{v}($ gross $)=4128 \mathrm{cal} \mathrm{g}^{-1}$
maximum $q_{v}($ gross $)=17270 \mathrm{~J} \mathrm{~g}^{1}$
average $q_{v}($ gross $)=2597 \mathrm{cal} \mathrm{g}^{-1}$
average $q_{v}($ gross $)=10867 \mathrm{~J} \mathrm{~g}^{1}$

(batch 2)

minimum $q_{v}$ (gross) $=1275 \mathrm{cal}^{-1}$

minimum $q_{v}^{v}($ gross $)=5333 \mathrm{~J} \mathrm{~g}^{-1}$

maximum $q_{v}($ gross $)=4221 \mathrm{cal} \mathrm{g}^{-1}$

maximum $q_{v}^{v}$ (gross) $=17661 \mathrm{~J} \mathrm{~g}^{2}$

average $q_{v}($ gross $)=2789 \mathrm{cal} \mathrm{g}^{-1}$

average $q_{v}($ gross $)=11669 \mathrm{~J} \mathrm{~g}^{2}$

REFUSE, MUNICIPAL; solid; waste from an English town; (sample 1) (on dry basis); paper, $29.6 \%$; rags, $5.3 \%$; vegetable matter, $5.1 \%$; animal matter $1.3 \%$; wood, etc., $3.5 \%$; coal, coke, cinders, $16.5 \%$; metal, $4.9 \%$; glass, pottery, and other non-combustible matter, $23.7 \%$; fines, passing $1 / 4 \times 1 / 4 "$ square mesh, $10.1 \%$; moisture, $34.0 \%$; (sample 2) (on dry basis) paper, $4.8 \%$, rags, $0.3 \%$; vegetable matter $1.0 \%$; animal matter, $0.7 \%$; wood, etc., $0.8 \%$; coal, coke, cinders, $36.1 \%$; metal $0.9 \%$; glass pottery, and other non-combustible material, $10.0 \%$; fines, passing $1 / 4 \times 1 / 4 "$ square mesh, $45.4 \%$; moisture, $17.2 \%$.

gross heat of combustion: assume values refer to room temperature; dry basis. sample 1

$q_{y}$ (gross) $=3110 \mathrm{cal}^{-1}$
$q_{v}($ gross $)=13012 \mathrm{~J} \mathrm{~g}^{-1}$

sample 2

$q_{v}($ gross $)=4060 \mathrm{cal} \mathrm{g}^{-1}$
$q_{v}($ gross $)=16987 \mathrm{~J} \mathrm{~g}^{1}$ 
RUBBER; solid; (sample 1) latex, digested with water at $190^{\circ} \mathrm{C}$, extracted with water and ethanol; soluble in ether; $0.02 \%$ ash; (sampie 2) latex, trypsin treated; is\% ether soluble, $25 \%$ ether insoluble.

gross heat of combustion: $30^{\circ} \mathrm{C}$.

sample $1,0.02 \%$ ash

$q_{v}$ (gross) $=10814 \mathrm{cal} \mathrm{g}^{-1}$
$q_{v}$ (gross) $=45247 \mathrm{~J} \mathrm{~g}$

sample $2,0.4 \%$ ash

$q_{v}$ (gross) $=10753 \mathrm{cal}^{-1} \mathrm{~g}^{-1}$
$q_{v}($ gross $)=44991 \mathrm{Jg}^{-1}$

sample 2, $0.15 \%$ ash

$q_{v}$ (gross) $=10784 \mathrm{cal}^{-g^{-1}}$
$q_{v}($ gross $)=45120 \mathrm{Jg}^{-1}$

sample 2, ether soluble fraction, $0.1 \%$ ash

$q_{v}$ (gross) $=10802 \mathrm{cal}_{-\mathrm{g}^{-1}}$
$q_{v}($ gross $)=45196 \mathrm{~J} \mathrm{~g}^{-1}$

sample 2 , ether insoluble fraction, $0.7 \%$ ash

$q_{v}($ gross $)=10746 \mathrm{cal} \mathrm{g}^{-1}$
$q_{v}$ (gross) $=44961 \mathrm{Jg}^{-1}$

sample 2, ether insoluble fraction, $1.8 \%$ ash

$q_{v}($ gross $)=10627 \mathrm{cal} \mathrm{g}^{-1}$
$q_{v}($ gross $)=44464 \mathrm{Jg}^{-1}$

RUBBER-SULFUR COMPOUNDS; solid; repeating unit: $\left[\left(\mathrm{C}_{5} \mathrm{H}_{8}\right)_{x} \mathrm{~S}_{y}\right]$; $x$ varies between 1 and 4 ; $y$ varies between 0 and 3 ; maximum sulfur content $\sim 32 \%$.

gross heat of combustion: at $30{ }^{\circ} \mathrm{C}$, sulfur as gaseous $\mathrm{SO}_{2}$.

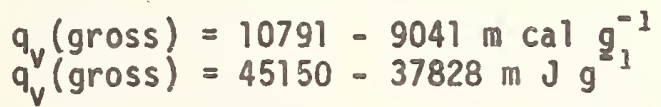

$m$ is grams of sulfur per gram of compound.

SANTOWAX R; liquid; (trade mark, C Monsanto Co.l; mixture of terphenyl hydrocarbons.

specific heat:

$$
\begin{aligned}
& 180-320^{\circ} \mathrm{C} . \\
& \begin{array}{ll}
C p\left(\mathrm{cal} g^{-1} \mathrm{~K}^{-1}\right) & =0.222+5.86 \times 10^{-4} \mathrm{~T} \\
C p\left(J g^{-1} K^{-1}\right) & =0.929+2.452 \times 10^{-3^{\top}} \mathrm{T}
\end{array}
\end{aligned}
$$


SEA WATER; aqueous; a uniform solution of essentially constant composition containing $96.5 \%$ water and $3.5 \%$ ionized salts, related compounds, elements, and ionic complexes; sodium chloride is the chief salt constituent; other common ions are: $\mathrm{K}, \mathrm{Mg}^{2}+\mathrm{Ca}^{2}, \mathrm{SO}_{4}{ }^{2-}, \mathrm{HCO}_{3}{ }^{-}$; salinity $(\mathrm{S})$ is defined as the number of grams of salt in one kilogram of sea water when bromides and iodides are converted to chlorides, the carbonates to oxides, organic matter destroyed, and the mass heated to $450{ }^{\circ} \mathrm{C}$ for 72 hours; $S=0.03+1.805$ (chloride content).

specific heat:

Temperature, ${ }^{\circ} \mathrm{C}$

$$
\left.C p(c a) g^{-1} K^{-1}\right) \quad C p\left(J g^{-1} K^{-1}\right)
$$

salinity, $0.0 \%$

$\begin{array}{rrr}0 & 1.0080 & 4.2175 \\ 20 & 0.9995 & 4.1819 \\ 50 & 0.9992 & 4.1807 \\ 100 & 1.0076 & 4.2158 \\ 200 & 1.0747 & 4.4965\end{array}$

salinity, $3 \%$

$\begin{array}{rll}0 & 0.9601 & 4.0171 \\ 20 & 0.9606 & 4.0192 \\ 50 & 0.9638 & 4.0325 \\ 100 & 0.9715 & 4.0648 \\ 200 & 1.0254 & 4.2903\end{array}$

salinity, $6 \%$

$\begin{array}{rll}0 & 0.9198 & 3.8484 \\ 20 & 0.9257 & 3.8731 \\ 50 & 0.9313 & 3.8966 \\ 100 & 0.9387 & 3.9275 \\ 200 & 0.9833 & 4.1141\end{array}$

salinity, $9 \%$

$\begin{array}{rrr}0 & 0.8874 & 3.7129 \\ 20 & 0.8944 & 3.7422 \\ 50 & 0.9009 & 3.7694 \\ 100 & 0.9079 & 3.7987 \\ 200 & 0.9450 & 3.9539\end{array}$

salinity, $12 \%$

$\begin{array}{rll}0 & 0.8637 & 3.6137 \\ 20 & 0.8670 & 3.6275 \\ 50 & 0.8729 & 2.6522 \\ 100 & 0.8791 & 3.6782 \\ 200 & 0.9096 & 3.8058\end{array}$


SHOE, LEATHER; solid; ultimate analysis in percent by weight; carbon, $42.01 \%$; hydrogen, $5.32 \%$; oxygen, $22.83 \%$; nitrogen, $5.98 \%$; sulfur, $1.00 \%$; ash, 22.86\%; proximate analysis as received in percent by weight; moisture, $7.46 \%$; volatile matter, $57.12 \%$; fixed carbon, $14.26 \%$; ash, $21.16 \%$.

gross heat of combustion: assume values refer to room temperature.

(as received)

$$
\begin{aligned}
& q_{v}(\text { gross })=4027 \mathrm{cal} \mathrm{g}_{1}^{-1} \\
& q_{v}(\text { gross })=16847 \mathrm{~J} \mathrm{~g}^{2} \\
& (\text { dry basis }) \\
& q_{v}(\text { gross })=4351 \mathrm{cal} \mathrm{g_{1 } ^ { - 1 }} \\
& q_{v}(\text { gross })=18203 \mathrm{~J} \mathrm{~g}^{-1}
\end{aligned}
$$

STEEL, ALLOY, NPL NO. 19; solid; composition: carbon, $0.315 \%$; silicon, $0.20 \%$; manganese, $0.69 \%$; sulfur, $0.036 \%$; phosphorous, $0.039 \%$; chromium, $1.09 \%$; nic el, 0.073\%; molybdenum, 0.012\%; copper, 0.066\%; aluminum, $0.005 \%$; arsenic, $0.028 \%$; remainder is iron; annealed at $860{ }^{\circ} \mathrm{C}$; density, 7.842 $\mathrm{g} \mathrm{\textrm {cm } ^ { - 3 }}$ at $15^{\circ} \mathrm{C}$.

specific heat:

Temperature $\left({ }^{\circ} \mathrm{C}\right)$

$$
\begin{array}{cc}
\left.C p(c a) g^{-1} K^{-1}\right) & C p\left(J g^{-1} K^{-1}\right) \\
0.1153 & .4824 \\
0.1232 & .5155 \\
0.1277 & .5343 \\
0.1354 & .5665 \\
0.1471 & .6155
\end{array}
$$$$
50
$$$$
127
$$$$
217
$$$$
316
$$$$
409
$$

STEEL, STAINLESS 347 ; solid; nicke1, $11.1 \%$; chromium, $18.3 \%$; manganese, $1.30 \%$; silicon, $0.52 \%$; carbon, $0.08 \%$; remainder is iron.

specific heat:

0 to $900{ }^{\circ} \mathrm{C}$

$\left.C p(c a) g^{-1} K^{-1}\right)=0.1158+3.41 \times 10^{-5} T-4.04 T^{-1}$

$\mathrm{Cp}\left(\mathrm{J} \mathrm{g}^{-1} \mathrm{~K}^{-1}\right)=0.4845+1.427 \times 10^{-4} \mathrm{~T}-16.90 \mathrm{~T}^{-1}$ 
STEEL, STAINLESS, 446; solid; nicke1, $0.32 \%$; chromium, $25.58 \%$; manganese, $0.42 \%$; silicon, 0.68\%; carbon, $0.23 \%$; remainder is iron.

\section{specific heat:}

0 to $500{ }^{\circ} \mathrm{C}$

$\begin{array}{ll}\operatorname{Cp}\left(\operatorname{cal} g^{-1} K^{-1}\right) & =0.0785+1.074 \times 10^{-4} \mathrm{~T} \\ \operatorname{Cp}\left(J g^{-1} K^{-1}\right) & =0.3284+4.494 \times 10^{-4} \mathrm{~T}\end{array}$

600 to $900{ }^{\circ} \mathrm{C}$

$C p\left(c a l g^{-1} K^{-1}\right)=0.1618+2209 \times 10^{-0.0078}(T-273.2)$

$\mathrm{Cp}\left(\mathrm{J} \mathrm{g}^{-1} \mathrm{~K}^{-1}\right)=0.6770+9242 \times 10^{-0.0078}(\mathrm{~T}-273.2)$

[97]

STEEL, STAINLESS; solid; En-58C (sample 1): Cr 17.7\%; Ni 10.9\%; Mn 1.73\%; En-58B (sample 2): $\operatorname{Cr~17.8\% ;~Ni~8.05\% ;~Mn~1.21\% .~}$

\section{specific heat:}

\section{Sample 1 (En-58C)}

$60-190 k$

$\left.C p(c a) g^{-1} K^{-1}\right)=-0.04976+1.7309 \times 10^{-3} T-6.980 \times 10^{-6} \mathrm{~T}^{2}+1.043 \times 10^{-8} \mathrm{~T}^{3}$ $\operatorname{Cp}\left(\mathrm{J} \mathrm{g} \mathrm{g}^{-1} \mathrm{~K}^{-1}\right)=-0.20819+7.2421 \times 10^{-3} \mathrm{~T}-2.9206 \times 10^{-5} \mathrm{~T}^{2}+4.364 \times 10^{-8} \mathrm{~T}^{3}$

$190-320 k$

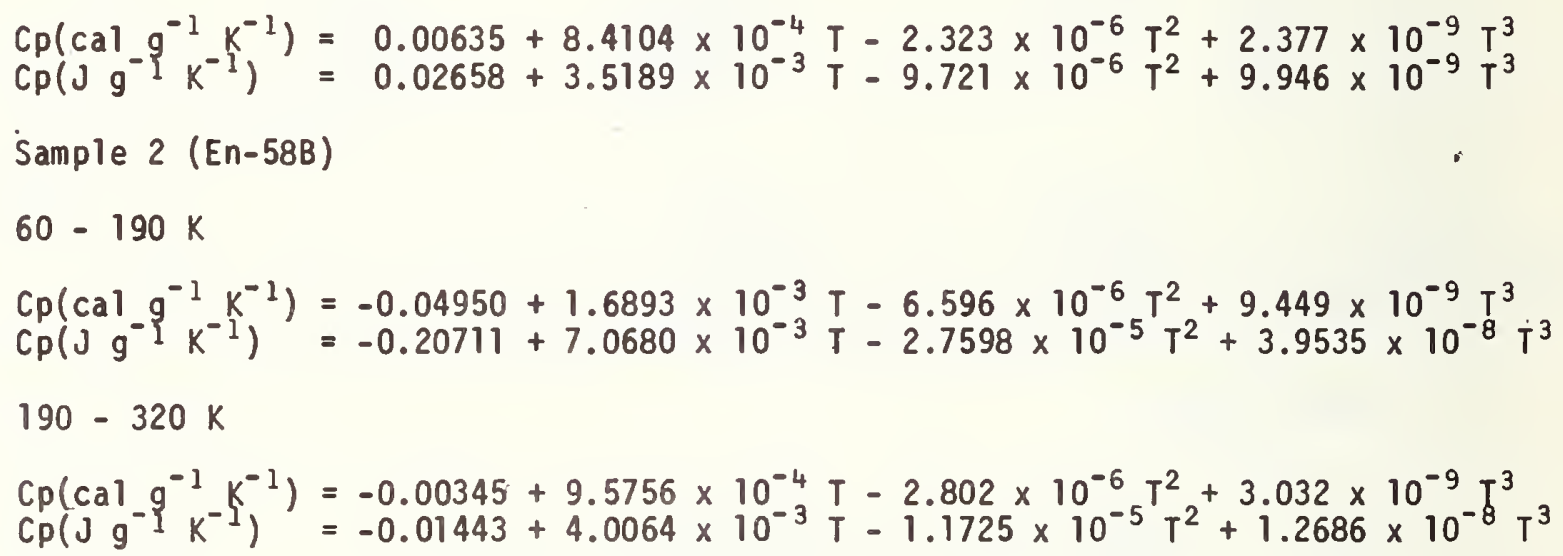


SULFITE LIQUOR, SPENT; aqueous; calcium and magnesium base waste liquor from pulping of wood by sulfite process; gaseous sulfur dioxide, 2-4\%; aqueous sulfite. 2-7\%: aqueous sulfate, $2-5 \%$; solids, $80-90 \%$; also lignin sulfonate, $\mathrm{Ca}$ and $\mathrm{Mg}$ compounds, and carbohydrates.

gross heat of combustion: assume value refers to room temperature.

$55 \%$ solids non-neutralized spent liquor

$q_{v}($ gross $)=4500-4650 \mathrm{cal} \mathrm{g}^{-1}$
$q_{v}($ gross $)=18800-19500 \mathrm{Jg}^{-1}$

fermented and stripped liquor

$q_{v}($ gross $)=4700-4875 \mathrm{cal} \mathrm{g}^{-1}$
$q_{v}($ gross $)=19700-20400 \mathrm{Jg}^{-1}$

TANNIN EXTRACT, CHESTNUT; Solid; dry powder obtained from concentrated solution produced from treatment of chestnut chips with water.

specific heat:

$25^{\circ} \mathrm{C}$

$\operatorname{Cp}\left(\mathrm{cal} g^{-1} K^{-1}\right)=0.2889$

$C p\left(J g^{-1} K^{-1}\right)=1.2088$

[78]

TANNIN EXTRACT, QUEBRACHO; solid; dry extract from a wood-derived tannin; tannin obtained from Aspidosperma Quebracho and Quebracho Lorentzi imported as logs from Argentina.

specific heat:

$25^{\circ} \mathrm{C}$

$C p\left(c a l g^{-1} K^{-1}\right)=0.3152$

$\mathrm{Cp}\left(\mathrm{J} \mathrm{g}^{-1} \mathrm{~K}^{-1}\right)=1.3188$ 
IIRE; solid; typical compounding formulae for passenger tire treads.

$$
\text { Natural rubber SBR cis-polybutadiene* }
$$

Smoked sheet

$57.6 \%$

SBR 1712

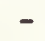

$46.8 \%$

cis-Polybutadiene

$-$

1.2

11.3

Reogen

$-$

$-$

K-Stay $G$

Stearic acid

Zinc oxide

Agerite resin D

1.4

2.3

2

0.9

Agerite HP

0.9

1.4

0.3

0.7

Antozite 675

Microcrystalline wax

2.3

0.2

Philrich 5

0.6

1.8

HAF

2.9

28.8

0.5

ISAF

Sulfur

1.4

3.2

$-$

Amax No. 1

0.3

29.5

Amax

$-$

Redax

0.3

0.8

0.7

*SBR is styrene-butadiene rubber

gross heat of decomposition: at $375{ }^{\circ} \mathrm{C}$ the mean value is for randoin samples of black-sidewall tire tread; products are a liquid distillate with a heat of combustion $q_{v}=4 \times 10^{4} \mathrm{~J} \mathrm{~g}^{-1}\left(1 \times 10^{4} \mathrm{cal} \mathrm{g}^{-1}\right)$ and a combustible gas.

$q_{v}($ grosis $)=0.7 \times 10^{2} \mathrm{cal} \mathrm{g}^{-1}$
$q_{v}($ gross $)=3 \times 10^{2} \mathrm{Jg}^{-1}$

gross heat of combustion: value for the tire residue remaining after heating to $1000{ }^{\circ} \mathrm{C}$.

$q_{v}($ gross $)=0.780 \times 10^{4} \mathrm{cal}^{-\mathrm{g}^{-1}}$
$q_{v}($ gross $)=3.264 \times 10^{4} \mathrm{~J} \mathrm{~g}^{-1}$

$[71]$

1OPAZ; solid, an aluminum fluorosilicate occuring in igneous rocks and pegniatites, often found in association with tin ores; $\mathrm{Al}_{2} \mathrm{SiO}_{4}(\mathrm{~F}, \mathrm{OH})_{2}$; natural colorless orthorhombic crystals.

specific heat:

Temperature, $\mathrm{K}$

80

100

200

300

400

$$
\left.C p(c a) g^{-1} K^{-1}\right)
$$

0.022

0.043

0.126

0.185

0.217

$$
\operatorname{Cp}\left(\mathrm{J} \mathrm{g}^{-1} \mathrm{k}^{-1}\right)
$$

0.092

0.180

0.527

0.174

0.908 
VACUUM CLEANER CATCH; solid; Ultimate analysis on dry basis in percent by weight;

carbon, $35.69 \%$; hydrogen, $4.73 \%$; oxygen, $20.08 \%$; nitrogen, $6.26 \%$; sulfur, $1.15 \%$; ash, $32.09 \%$; proximate analys is as received in percent by weight; moisture, $5.47 \%$; volatile matter, $55.68 \%$; fixed carbon, $8.51 \%$; ash, $30.34 \%$.

gross heat of combustion: assume values refer to room temperature.

(as received)

$q_{v}$ (gross) $=3550 \mathrm{cal}^{-1}$
$q_{v}$ (gross) $=14854 \mathrm{~J} \mathrm{~g}^{1}$

(dry basis)

$q_{v}$ (gross) $=3756 \mathrm{cal} \mathrm{g}^{-1}$

$q_{v}^{v}($ gross $)=15714 \mathrm{car}^{-1}$

VASELINE; solid; semi-solid mixture of hydrocarbons having a melting point range from 38 to $60^{\circ} \mathrm{C}$; colorless or pale yellow petroleum jelly.

gross heat of combustion: assume values refer to room temperature.

$q_{v}$ (gross) $=11050 \mathrm{cal}^{-1}$

$q_{v}($ gross $)=46233 \mathrm{~J} \mathrm{~g}^{-1}$

WAX, PARAFFIN; solid; hard wax, freezing point $53-55^{\circ} \mathrm{C}$; density $0.902 \mathrm{~g} \mathrm{~cm}^{-3}$; repeating unit: $\mathrm{CH}_{2}$.

gross heat of combustion: $25^{\circ} \mathrm{C}$

$q_{v}$ (gross) $=11049 \mathrm{cal}^{-g^{-1}}$
$q_{v}($ gross $)=46229 \mathrm{~J} \mathrm{~g}^{-1}$

WOOD

ASH; Fraxinus excelsior; solid; Uitimate analysis: carbon, $49.18 \%$; hydrogen, $6.27 \%$; nitrogen, $0.07 \%$; ash, $0.57 \%$.

gross heat of combustion: $20^{\circ} \mathrm{C}$; air dried at $110-115^{\circ} \mathrm{C}$, ash free.

$q_{v}($ gross $)=4726 \mathrm{car}^{-1} \mathrm{~g}_{1}$
$q_{v}($ gross $)=19774 \mathrm{~J} \mathrm{~g}^{2}$ 
ASH, WHITE; Fraxinus americana; solid; air dried at $105-110{ }^{\circ} \mathrm{C}$; density $0.63 \mathrm{~g} \mathrm{~cm}^{-3}$. specific heat:

$$
\begin{aligned}
& 0-106{ }^{\circ} \mathrm{C} \\
& \begin{array}{ll}
\left.C p(c a)-g^{-1} K^{-1}\right) & =0.327 \\
C p\left(J g g^{-1} K^{-1}\right) & =1.368
\end{array}
\end{aligned}
$$

ASPEN, QUAKING; Populus tremuloides Michx; solid; air dried at $105-110^{\circ} \mathrm{C}$, density $0.42 \mathrm{~g} \mathrm{~cm}^{-3}$.

specific heat:

$$
\begin{aligned}
& 0-106^{\circ} \mathrm{C} \\
& \left.C p(c a) g^{-1} k^{-1}\right)=0.329 \\
& C p\left(\mathrm{~J} \mathrm{~g}^{-1} \mathrm{~K}^{-1}\right)=1.377
\end{aligned}
$$

BEECH; Fagus silvatiea; solid; Ultimate analysis: carbon, $49.06 \%$; hydrogen, $6.11 \%$; 11 itrogen, $0.09 \% ; \mathrm{ash}, 0.57 \%$.

gross heat of combustion: $20{ }^{\circ} \mathrm{C}$, air dried at $110-115^{\circ} \mathrm{C}$, ash free.

$$
\begin{aligned}
& q_{v}(\text { gross })=4791 \mathrm{cal} \mathrm{g}^{-1} \\
& q_{v}(\text { gross })=20046 \mathrm{~J} \mathrm{~g}^{1}
\end{aligned}
$$
$\frac{\text { BEECH; Fagus atropunicea (Marsh) Sudworth; solid; air dried at } 105-110{ }^{\circ} \mathrm{C} \text {; density }}{0.75 \mathrm{~g} \mathrm{~cm}^{-3} \text {. }}$

specific heat:

$$
\begin{aligned}
& 0-106{ }^{\circ} \mathrm{C} \\
& C p\left(c a 1 g^{-1} K^{-1}\right)=0.326 \\
& C p\left(\mathrm{Jg}^{-1} \mathrm{~K}^{-1}\right)=1.364
\end{aligned}
$$


WOOD

BIRCH; Betula alba; solid; Ultimate analysis: carbon, $48.88 \%$; hydrogen, $6.06 \%$; nitrogen, $0.10 \% ; \mathrm{ash}, 0.29 \%$.

gross heat of combustion: $20^{\circ} \mathrm{C}$, air dried at $110-115^{\circ} \mathrm{C}$, ash free.

$q_{v}($ gross $)=4775 \mathrm{cal} \mathrm{g}^{-1}$
$q_{v}($ gross $)=19979 \mathrm{~J} \mathrm{~g}^{2}$

species not identified: $10.18 \%$ moisture.

$q_{v}($ gross $)=4224 \mathrm{cal} \mathrm{g}^{-1}$
$q_{v}($ gross $)=17672 \mathrm{~J} \mathrm{~g}^{1}$

air dried one hour at $105^{\circ} \mathrm{C}$

$q_{v}$ (gross) $=4703 \mathrm{cal} \mathrm{g}^{-1}$

$q_{v}^{v}($ gross $)=19677 \mathrm{Jg}^{3}$

CEDAR, RED; Juniperus virginiana L.; solid; air dried at $105-110^{\circ} \mathrm{C}$; density $0.46 \mathrm{~g} \mathrm{~cm}^{-3}$. specific heat:

$0-106 \cdot{ }^{\circ} \mathrm{C}$

$\operatorname{Cp}\left(\operatorname{cal} g^{-1} K^{-1}\right)=0.324$
$\operatorname{Cp}\left(J g g^{-1}\right)=1.356$

[139]

CHERRY; solid; genus Prunus.

gross heat of combustion: assume values refer to room temperature.

as received: $8.85 \%$ moisture.

$q_{v}($ gross $)=4370 \mathrm{cal} \mathrm{g}^{-1}$
$q_{v}($ gross $)=18282 \mathrm{~J} \mathrm{~g}^{1}$

air dried one hour at $105^{\circ} \mathrm{C}$

$q_{v}($ gross $)=4794 \mathrm{cal} \mathrm{g}^{-1}$
$q_{v}($ gross $)=20058 \mathrm{~J} \mathrm{~g}^{1}$ 
CHESTNUT; Castanea dentata (Marsh) Borkh; solid; air dried at $105-110^{\circ} \mathrm{C}$; density $0.32 \mathrm{~g} \mathrm{~cm}^{-3}$.

specific heat:

$0-106{ }^{\circ} \mathrm{C}$

$\operatorname{Cp}\left(\mathrm{cal} q^{-1} \mathrm{~K}^{-1}\right)=0.317$

$C p\left(\mathrm{Jg}^{-1} \mathrm{~K}^{-1}\right)=1.326$

[139]

COCOBOLA NEGRA; Lecythis costariconsis Pittier; solid; from Costa Rica; air dried at $105-110^{\circ} \mathrm{C}$; density $0.92 \mathrm{~g} \mathrm{~cm}^{-3}$.

Specific heat:

$0-106{ }^{\circ} \mathrm{C}$

$\begin{array}{ll}C p\left(c a l g^{-1} K^{-1}\right) & =0.327 \\ C p\left(J g g^{-1} K^{-1}\right) & =1.368\end{array}$

[139.]

ELM, WHITE; Ulmus americana L.; solid; air dried at $105-110^{\circ} \mathrm{C}$; density $0.64 \mathrm{~g} \mathrm{~cm}^{-1}$. specific heat:

$$
\begin{aligned}
& 0-106{ }^{\circ} \mathrm{C} \\
& \begin{array}{ll}
C p\left(\mathrm{cal} \mathrm{g}^{-1} \mathrm{~K}^{-1}\right) & =0.325 \\
\operatorname{Cp}\left(\mathrm{J} \mathrm{g} \mathrm{g}^{-1} \mathrm{~K}^{-1}\right) & =1.360
\end{array}
\end{aligned}
$$

FIR; Pinus silvestri S.; solid; Ultimate analysis: carbon, $50.36 \%$; hydrogen, $5.92 \%$; nitrogen, $0.05 \%$; ash, $0.28 \%$.

gross heat of combustion: $20^{\circ} \mathrm{C}$; air dried at $110-115^{\circ} \mathrm{C}$, ash free.

$$
\begin{aligned}
& q_{v}(\text { gross })=5038 \mathrm{cal} \mathrm{g}^{-1} \\
& q_{v}(\text { gross })=21079 \mathrm{~J} \mathrm{~g}^{1}
\end{aligned}
$$

FIR, DOUGLAS; Pseudotsuga taxifolia (Lam.) Britt; solid; air dried at $105-110^{\circ} \mathrm{C}$. density $0.48 \mathrm{gm} \mathrm{cm}^{-3}$.

$$
\begin{aligned}
& C p\left(c a l g^{-1} k^{-1}\right)=0.327 \\
& C p\left(J g^{-1} K^{-1}\right)=1.368
\end{aligned}
$$


GUM, BLACK; Nyssa sylvatica Marsh; solid; air dried at $105-110^{\circ} \mathrm{C}$; density $0.52 \mathrm{~g} \mathrm{~cm}^{-3}$. specific heat:

$0-106{ }^{\circ} \mathrm{C}$

$\begin{array}{ll}\operatorname{Cp}\left(\operatorname{cal} g^{-1} K^{-1}\right) & =0.325 \\ \operatorname{Cp}\left(J g^{-1} K^{-1}\right) & =1.360\end{array}$

HEMLOCK, WESTERN; Tsuga heterophylla (Raf.) Sargent; solid; air dried at $105-110^{\circ} \mathrm{C}$; density $0.45 \mathrm{~g} \mathrm{~cm}^{-3}$.

specific heat:

$0-106^{\circ} \mathrm{C}$

$\begin{array}{ll}\operatorname{Cp}\left(\operatorname{cal} g^{-1} K^{-1}\right) & =0.322 \\ \operatorname{Cp}\left(J g g^{-1} K^{-1}\right) & =1.347\end{array}$

gross heat of combustion: assume values refer to room temperature; air dried.

$q_{v}($ gross $)=4792 \mathrm{cal} \mathrm{g}^{-1}$
$q_{v}($ gross $)=20050 \mathrm{Jg}^{1}$

HICKORY; solid; genus Carya.

gross heat of combustion: assume values refer to room temperature.

as received: $10.30 \%$ moisture.

$q_{v}($ gross $)=4213 \mathrm{cal} \mathrm{g}^{-1}$
$q_{v}($ gross $)=17626 \mathrm{j} \mathrm{g}_{1}$

air dried one hour at $105^{\circ} \mathrm{C}$.

$q_{v}($ gross $)=4697 \mathrm{cal} \mathrm{g}^{-1}$
$q_{v}($ gross $)=19652 \mathrm{~J} \mathrm{~g}^{1}$

HORNBEAM; Carpinus betulus; solid; Ultimate analysis: carbon, $48.99 \%$; hydrogen, $6.20 \%$; nitrogen, $0.06 \%$; ash, $0.50 \%$.

yross heat of combustion: $20^{\circ} \mathrm{C}$, air dried $110-115^{\circ} \mathrm{C}$, ash free.

$q_{v}($ gross $)=4742 \mathrm{cal} \mathrm{g}^{-1}$
$q_{v}($ gross $)=19841 \mathrm{~J} \mathrm{~g}^{2}$ 
MAI CHAMPAH; Michelia; solid; from Thailand; air dried at $105-110^{\circ} \mathrm{C}$; density $0.32 \mathrm{~g} \mathrm{~cm}^{-3}$. specific heat:

$0-106{ }^{\circ} \mathrm{C}$

$C p\left(\operatorname{cal} g^{-1} K^{-1}\right)=0.323$

$C p\left(\mathrm{~J} \mathrm{~g}^{-1} \mathrm{~K}^{-1}\right)=1.351$

MAPLE; solid; Ultimate analysis: carbon, $50.40 \%$; hydrogen, $5.90 \%$; oxygen, $39.10 \%$; nitrogen, $0.50 \%$; sulfur, $0.0 \%$; ash, $4.10 \%$; proximate analys is as received in percent by weight; moisture, $0.0 \%$; volatile matter, $76.1 \%$; fixed carbon, $19.6 \%$; ash, $4.3 \%$; genus, Acer.

gross heat of combustion: assume values refer to room temperature.

$q_{v}($ gross $)=4553 \mathrm{cal} \mathrm{g}^{-1}$
$q_{v}($ gross $)=19050 \mathrm{~J} \mathrm{~g}^{1}$

$[84,85]$

MAPLE, SUGAR; Acer saccharum Marsh; solid; air dried at $105-110^{\circ} \mathrm{C}$; density $0.66 \mathrm{~g} \mathrm{~cm}^{-3}$.

specific heat:

$0-106{ }^{\circ} \mathrm{C}$

$C_{p}\left(\operatorname{cal} g^{-1} K^{-1}\right)=0.327$

$\mathrm{Cp}\left(\mathrm{J} \mathrm{g}^{-1} \mathrm{~K}^{-1}\right)=1.368$

OAK; Quereus pedunculata; U1timate analysis: solid; carbon, $50.16 \%$; hydrogen, $6.02 \%$; nitrogen, 0.09\%; ash, $0.37 \%$.

gross heat of combustion: $20^{\circ} \mathrm{C}$; air dried at $110-115^{\circ} \mathrm{C}$; ash free.

$q_{v}($ gross $)=4609 \mathrm{cal} \mathrm{g}^{-1}$
$q_{v}($ gross $)=19284 \mathrm{~J} \mathrm{~g}^{-1}$

species not identified; room temperature; air dried one hour at $105{ }^{\circ} \mathrm{C}$.

$q_{v}($ gross $)=4756 \mathrm{cal} \mathrm{g}^{-1}$
$q_{v}($ gross $)=19899 \mathrm{~J} \mathrm{~g}^{1}$ 
OAK, RED; Quercus rubra (L); solid; air dried at $105-110^{\circ} \mathrm{C}$, density $0.62 \mathrm{~g} \mathrm{~cm}^{-3}$ specific heat:

$0-106^{\circ} \mathrm{C}$

$\operatorname{Cp}\left(\operatorname{cal} g^{-1} K^{-1}\right)=0.331$
$\operatorname{Cp}\left(J g g^{-1} K^{-1}=1.385\right.$

OAK, WHITE; Quercus alba (L); solid; air dried at $105-110^{\circ} \mathrm{C}$, density $0.78 \mathrm{~g} \mathrm{~cm}^{-3}$.

specific heat:

$0-106^{\circ} \mathrm{C}$

$\begin{array}{ll}\operatorname{Cp}\left(\operatorname{cal} g^{-1} K^{-1}\right) & =0.325 \\ \operatorname{Cp}\left(J^{-1} K^{-1}\right) & =1.360\end{array}$

[139]

PINE, LOBLOLLY; Pinus taeda L.; solid; unextracted specific gravity, 0.49 (oven dry weight and green volume).

specific heat: $60-140^{\circ} \mathrm{C}$; samples were oven-dried at $100^{\circ} \mathrm{C}$ for 12 hours before measurements were made.

$\begin{array}{ll}C p\left(\operatorname{cal} g^{-1} K^{-1}\right) & =0.01153+9.497 \times 10^{-4} T \\ C p\left(J g g^{-1} K^{-1}\right) & =0.04824+3.9735 \times 10^{-3} T\end{array}$

PINE, LOBLOLLY (Pinus taeda L.), EXTRACTIVES; solid; residue obtained from alcoholbenzene extraction of loblolly pine wood; mean extractive content, $6.08 \%$ [81].

specific heat: $60-140^{\circ} \mathrm{C}$; samples were oven-dried at $100^{\circ} \mathrm{C}$ for 12 hours before measurements were made.

$\begin{array}{ll}C p\left(c_{1} g^{-1} K^{-1}\right) & =0.06945+1.09 \times 10^{-3} \mathrm{~T} \\ C p\left(J g g^{-1} K^{-1}\right) & =0.29058+4.56 \times 10^{-3} \mathrm{~T}\end{array}$

PINE, LONGLEAF; Pinus palustris Mill; solid; air dried at $105-110^{\circ} \mathrm{C}$, density $0.68 \mathrm{~g} \mathrm{~cm}^{-3}$.

specific heat:

$0-106{ }^{\circ} \mathrm{C}$

$\left.C p(C a) g^{-1} K^{-1}\right)=0.337$

$\operatorname{Cp}\left(\mathrm{J} \mathrm{g}^{-1} \mathrm{~K}^{-1}\right)=1.410$ 
PINE, PONDEROSA; Pinus ponderosa; solid; samples were conditioned to moisture equilibrium at $80{ }^{\circ} \mathrm{F}$ and $30 \%$ relative humidity; moisture content of wood $26 \%$.

gross heat of combustion: assume values refer to room temperature.

$q_{v}($ gross $)=4626 \pm 38 \mathrm{cal} \mathrm{g}^{-1}$
$q_{v}($ gross $)=19355 \pm 159 \mathrm{Jg}^{-1}$

$[86,87]$

PINE, WHITE; Pinus strobus L.; solid; air-dried at $105-110^{\circ} \mathrm{C}$; density $0.25 \mathrm{~g} \mathrm{~cm}^{-3}$.

specific heat:

$0-106{ }^{\circ} \mathrm{C}$

$\operatorname{cp}\left(\operatorname{cal} g^{-1} K^{-1}\right)=0.331$
$\operatorname{Cp}\left(J g^{-1} K^{-1}\right)=1.385$

[139]

sawdust; proximate analysis, as delivered: moisture, $7.0 \%$; volatile matter, $78.76 \%$; fixed carbon, $14.10 \%$; ash, $0.14 \%$.

gross heat of combustion: air dried; assume values refer to room temperature.

$q_{v}($ gross $)=4592 \mathrm{cal} \mathrm{g}^{-1}$
$q_{v}($ gross $)=19213 \mathrm{~J} \mathrm{~g}^{1}$

$[84,85]$

POPLAR; solid; genus Populus.

gross heat of combustion: assume values refer to room temperature.

as received:

$10.69 \% \mathrm{H}_{2} \mathrm{O}$

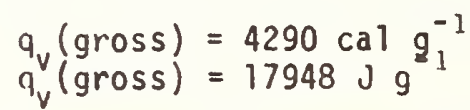

air-dried one hour at $105^{\circ} \mathrm{C}$.

$q_{v}($ gross $)=4803 \mathrm{cal} \mathrm{g}^{-1}$
$q_{v}($ gross $)=20096 \mathrm{~J} \mathrm{~g}^{2}$ 
SPRUCE, BALSAM; Abies Lasiocarpa; solid; U1timate analysis: carbon, $51.41 \%$; hydrogen, $6.41 \%$; nitrogen, $1.44 \%$; sulfur, $0.19 \%$; ash, $3.07 \%$; moisture, $3.67 \%$; proximate analysis as received: moisture, $74.35 \%$; volatile matter, $20.20 \%$; fixed carbon, $4.13 \%$; ash, $0.82 \%$.

gross heat of combustion: assume values refer to room temperature.

air-dried at $80^{\circ} \mathrm{C}$ :

$q_{v}($ gross $)=5304 \mathrm{cal} \mathrm{g}^{-1}$
$q_{v}($ gross $)=22191 \mathrm{~J} \mathrm{~g}^{-1}$

as received:

$q_{v}($ gross $)=1360 \mathrm{cal} \mathrm{g}^{-J}$
$q_{v}($ gross $)=5692 \mathrm{~J} \mathrm{~g}^{-1}$

SPRUCE, NORWAY; Pinus abies; solid; carbon, $50.31 \%$; hydrogen, $6.20 \%$; nitrogen, $0.04 \%$; ash, $0.37 \%$.

gross heat of combustion: at $20^{\circ} \mathrm{C}$; air-dried at $110-115^{\circ} \mathrm{C}$; ash free.

$q_{v}($ gross $)=5093 \mathrm{cal} \mathrm{g}^{-1}$
$q_{v}($ gross $)=21309 \mathrm{Jg}^{2}$

[89]

SPRUCE, RED; Picea rubens Sargent; solid; air-dried at $105-110^{\circ} \mathrm{C}$; density $0.39 \mathrm{~g} \mathrm{~cm}^{-3}$. specific heat:

$0-106{ }^{\circ} \mathrm{C}$

$\operatorname{Cp}\left(\mathrm{cal} \mathrm{g}^{-1} \mathrm{~K}^{-1}\right)=0.332$
$\operatorname{Cp}\left(\mathrm{J} \mathrm{g}^{-1} \mathrm{~K}^{-1}\right)=1.389$

[139]

SPRUCE PINE; Pinus glabra Walt.; solid; samples were dried for 8 hours in a vacuum oven at $103^{\circ} \mathrm{C}$.

specific heat:

60 to $140^{\circ} \mathrm{C}$

$C p\left(c a 1-g^{-1} k^{-1}\right)=-0.0091+1.004 \times 10^{-3} \mathrm{~T}$
$C p\left(J g^{-1} K^{-1}\right)=-0.0389+4.201 \times 10^{-3} \mathrm{~T}$ 
SPRUCE PINE, BARK; Pinus glabra Walt.; solid; bark specimens oven-dried at $152{ }^{\circ} \mathrm{C}$. specific heat:

60 to $140{ }^{\circ} \mathrm{C}$

$\operatorname{Cp}\left(\operatorname{cal} g^{-1} K^{-1}\right)=0.7859-3.0645 \times 10^{-3} \mathrm{~T}+5.137 \times 10^{-6} \mathrm{~T}^{2}$
$\operatorname{Cp}\left(J g^{-1} K^{-1}\right)=3.2882-1.2822 \times 10^{-2} \mathrm{~T}+2.1493 \times 10^{-5} \mathrm{~T}^{2}$

WOOL; solid; staple fibers obtained from the fleece of sheep (and also alpaca, vicuna, and certain goats); protein chains (Keratin) bound together by disulfide cross 1 inkages; chief amino acids: cysteine and cystine, $15 \%$; glutamic acid, 16\%; arginine, 10\%; proline, $8 \%$; leucine, $8 \%$; sulfur content of wool sample, $3.5 \%$.

gross heat of combustion: assume values refer to room temperature; dry basis, sulfur product aqueous $\mathrm{H}_{2} \mathrm{SO}_{4}$.

$q_{v}($ gross $)=5499 \mathrm{cal} \mathrm{g}^{-1}$
$q_{v}($ gross $)=23008 \mathrm{~J} \mathrm{~g}^{1}$

WOOL ACETATE; solid; salt formed from reaction of wool with acetic acid; $0.30 \%$ acetate. gross heat of combustion: assume values refer to room temperature; sulfur product aqueous $\mathrm{H}_{2} \mathrm{SO}_{4}$.

$q_{v}($ gross $)=5490 \mathrm{cal} \mathrm{g}^{-1}$
$q_{v}($ gross $)=22970 \mathrm{~J} \mathrm{~g}^{2}$

WOOL B-NAPHTHALENE SULFONATE; solid; salt formed from reaction of wool with B-naphthalenesulfonic acid; $19.9 \%$ B-naphthalenesulfonate.

gross heat of combustion: assume values refer to room temperature; sulfur product aqueous $\mathrm{H}_{2} \mathrm{SO}_{4}$.

$q_{v}($ gross $)=5482 \mathrm{cal} \mathrm{g}^{-1}$
$q_{v}($ gross $)=22937 \mathrm{~J} \mathrm{~g}^{1}$

WOOL OXAIAIE; solid; salt formed from reaction of wool with oxalic acid; $2.22 \%$ oxalate.

gross heat of combustion: assume values refer to room temperature; sulfur product aqueous $\mathrm{H}_{2} \mathrm{SO}_{1,}$.

$q_{v}($ gross $)=5360 \mathrm{cal} \mathrm{g}^{-1}$
$q_{v}($ gross $)=22426 \mathrm{~J} \mathrm{~g}^{1}$ 
WOOL PICRATE; solid; salt formed from reaction of wool with picric acid; 18.4 , picpite.

gross heat of combustion: assume values refer to room temperature; sulfur product aqueous $\mathrm{H}_{2} \mathrm{SO}_{4}$.

$q_{v}($ gross $)=5043 \mathrm{cal} \mathrm{g}^{-1}$
$q_{v}($ gross $)=21100 \mathrm{~J} \mathrm{~g}^{1}$

WOOL SULFATE; solid; salt formed from reaction of wool with sulfuric acid, $2.21 \%$ sulfate.

gross heat of combustion: assume values refer to room temperature, sulfur product aqueous $\mathrm{H}_{2} \mathrm{SO}_{4}$.

$q_{v}($ gross $)=5347 \mathrm{cal} \mathrm{g}^{-1}$
$q_{v}($ gross $)=22372 \mathrm{~J} \mathrm{~g}^{1}$

WOOL p-TOLUENESULFONATE; solid; salt formed from reaction of wool with p-toluenesulfonic acid; $6.6 \%$-toluenesuifonate.

gross heat of cor.bustion: assume values refer to room temperature; sulfur product aqueous $\mathrm{H}_{2} \mathrm{SO}_{4}$.

$q_{v}($ gross $)=5458 \mathrm{cal} \mathrm{g}^{-1}$
$q_{v}($ gross $)=22836 \mathrm{~J} \mathrm{~g}^{1}$

ZEOLITE, SILVER A; solid; synthetic ion-exchange resin; hydrated aluminum silicates containing alkali or alkaline earth metals; $90 \% \mathrm{Ag}^{+}$relative to $\mathrm{Na}^{+}$.

specific heat: at $310 \mathrm{~K}$; nearly all water eliminated by heating in $N_{2}$ at $500^{\circ} \mathrm{C}$.

$\operatorname{Cp}\left(\operatorname{cal} g^{-1} K^{-1}\right)=0.205$
$\operatorname{Cp}\left(J g^{-1} K^{-1}\right)=0.858$

[96]

ZEOLITE, SODIUM A; solid; synthetic ion-exchange resin; hydrated aluminum silicates containing alkali or alkaline earth metals.

specific heat: at $310 \mathrm{~K}$; nearly all water eliminated by heating in $\mathrm{N}_{2}$ at $500{ }^{\circ} \mathrm{C}$.

$\operatorname{Cp}\left(\operatorname{cal}_{\left.-g^{-1} K^{-1}\right)}=0.227\right.$
$\operatorname{Cp}\left(J g^{-1} K^{-1}\right)=0.950$ 
Acrylonitrile-styrene-polybutadiene copolymer, specific heat, 88

Alaska naphtha, heat of combustion, 54

Albumin, egg, heat of combustion, 38

Albumin, egg, specific heat, 38

Algae, heat of combustion, 69

Alloy, brass, specific heat, 12

Alloy, Hastelloy $x$, heat content, 1

Alloy, Hastelloy $X$, specific heat, 1

Alloy, 80 nickel-20 chromium, specific heat, 1

Alloy, titanium, Ti-75A, specific heat, 2

Alloy, titanium, Ti-150A, specific heat, 2

Alloy, Zircaloy-2, heat of transition, 2

Alloy, Zircaloy-2, specific heat, 2

Alloy, Zircaloy-2, (low nickel), specific heat, 3

Almonds, heat of combustion, 36

Animal (s), 3

Animal carcass, sheep, heat of combustion, 4

Animal fat, heat of combustion, 38

Animal fat, bovine, heat of combustion, 5,39

Animal fat, lard, heat of combustion, 39

Animal protein, bovine, heat of combustion, 5

Amatol, explosion temperature, 19

Amatol, heat of combustion, 19

Amatol, heat of explosion, 19

Amatol, specific heat, 19

Anthracite coal, heat of combustion, 14

Apiezon $C$ oil, heat of vaporization, 60

Apiezon C oil, vapor pressure, 60

Apiezon $E$ 0 11 , heat of vaporization, 61

Apiezon E oil, vapor pressure, 61

Apiezon $N$ grease, specific heat, 53

Apiezon $P$ grease, heat of vaporization, 54

Apiezon $P$ grease, vapor pressure, 54

Apiezon $R$ grease, heat of vaporization, 54

Apiezon $R$ grease, vapor pressure, 54

Apiezon $S$ grease, heat of vaporization, 54

Apiezon $S$ grease, vapor pressure, 54

Apiezon $T$ grease, glass temperature, 55

Apiezon $T$ grease, specific heat, 55

Aquatic plants, heat of combustion, 71

Aquatic snail, heat of combustion, 9

Arachnid(s) (see Animal(s))

Aromatic naphtha, heat of combustion, 59

Ash (tree), heat of combustion, 105

Ash, white (tree), specific heat, 106

Aspen, quaking, specific heat, 106

Asphalt, specific heat, 10

Automobile tire, heat of thermal decomposition, 104

Aviation fuel, heat of combustion, 48

Balsam spruce, heat of combustion, 113

Baratol, heat of fusion, 20

Baratol, specific heat, 20

Bark, spruce pine, specific heat, 114

Barley fat, heat of combustion, 38

Barnacle, heat of combustion, 3

Baronal, explosion temperature, 20

Barunal, heat of combustion, 20

Earonal, heat of explosion, 20

Basalt, vesicular, Iunar, specific heat, 58

Beans, navy, heat of combustion, 36 
Beech, heat of combustion, 106

Beech, specific heat, 106

Beef fat, heat of combustion, 5,39

Beetle, heat of combustion, 3

Beets, heat of combustion, 36

Bipheny1, polychlorinated, Clophen $A 30$, heat of vaporization, 10

Biphenyl, polychlorinated, Clophen $A 30$, vapor pressure, 10

Biphenyl, polychlorinated, Clophen $A 40$, heat of vaporization, 11

Biphenyl, polychlorinated, Clophen $A 40$, vapor pressure, 11

Bipheny1, polychlorinated, Clophen A50, heat of vaporization, 11

Biphenyl, polychlorinated, Clophen $\mathrm{A} 50$, vapor pressure, 11

Birch, heat of combustion, 107

Bituminous coal, heat of combustion, 15

Bivalve, heat of combustion, 3

Black gum (tree), specific heat, 104

Black powder, heat of explosion, 20

Blasting gelatine, heat of combustion, 21

Blood, sheep, heat of combustion, 4

Bovine animal fat, heat of combustion, 5,39

Bovine chymotrypsinogen $A$, specific heat, 97

Bovine protein, heat of combustion, 5

Bovine zinc insulin, specific heat, 55

Boxes, corrugated paper, heat of combustion, 66

Brachiopod, heat of combustion, 4

Brass, specific heat, 12

Brine shrimp, heat of combustion, 8

Brown coal, heat of combustion, 58

Brown paper, heat of combustion, 66

Butadiene-styrene copolymer, GL-622, specific heat, 73

Butadiene-styrene. copolymer, $X-454$, specific heat, 73

Butadiene-styrene copolymer, $X-478$, specific heat, 74

Butene(iso) polysulfone, heat of combustion, 75

Butene-1-polysulfone, heat of combustion, 74

Butene-2-polysulfone, heat of combustion, 74

Butterfat, heat of combustion, 39

Cabbage, heat of combustion, 37

Caddis fly, heat of combustion, 6

Cannel coal, heat of combustion, 17

Carbohydrate(s) (see Polysaccharide(s))

Carcass, sheep, heat of combustion, 4

Cartons, food, heat of combustion, 66

Cartons, waxed milk, heat of combustion, 68

Casein, milk, heat of combustion, 94

Cast iron, heat content, 56

Castor oil, specific heat, 61

Cattle, heat of combustion, 5

Cedar, red, specific heat, 107

Celluloid, heat of combustion, 13

$\alpha$-Cellulose, specific heat, 91

Cellulose, heat of combustion, 90

Cellulose diacetate, heat of combustion, 91

Cellulose nitrate, heat of combustion, 21

Cellulose nitrate, specific heat, 21

Cellulose triacetate, heat of combustion, 91

Cement hydrates, heat of dehydration, 13

Charcoal, wood, heat of combustion, 14

Cherry (tree), heat of combustion, 107

Chestnut (tree), specific heat, 108

Chestnut tannin extract, specific heat, 103

Chiton, heat of combustion, 5

Chromium-nickel alloy, specific heat, 1

Ciliate, heat of combustion, 5 
Citrus fruit, rinds and seeds, heat of combustion, 37

C? adocera, heat of combustion, 5

Clam, heat of combustion, 3

$\mathrm{Clophen} A 30$, heat of vaporization, 10

$\mathrm{Clophen} A 30$, vapor pressure, 10

$\mathrm{Clophen} \mathrm{A} 40$, heat of vaporization, 11

Clophen A40, vapor pressure, 11

Clophen A50, heat of vaporization, 11

Clophen $\mathrm{A50}$, vapor pressure, 11

Coal, anthracite, heat of combustion, 14

Coal, bituminous, heat of combustion, 15,16

Coal, brown, heat of combustion, 58

Coal, cannel, heat of combustion, 17

Coal, semi-anthracite, heat of combustion, 14

Coal, semi-bituminous, heat of combustion, 16

Coal, sub-bituminous, heat of combustion, 16

Cocobola negra, specific heat, 108

Coconut meat, heat of combustion, 37

Coconut oil, heat of combustion, 44

Coke, heat of combustion, 17

Coke breeze, heat of combustion, 18

Collagen, specific heat, 95

Combustion, heat of (see Heat of combustion)

Composition B, RDX-TNT explosive, explosion temperature, 22

Composition B, RDX-TNT explosive, heat of combustion, 22

Composition B, RDX-TNT explosive, heat of explosion, 22

Composition B, RDX-TNT explosive, heat of fusion, 22

Composition B, RDX-TNT explosive, specific heat, 22

Compressibility, crude oil, 62

Compressibility, gas, natural, 49

Compressibility, natural gas, 49

Copepod, heat of combustion, 6

Corn (maize), green, heat of combustion, 37

Corn (maize) meal, heat of combustion, 43

Corn (maize) oil, heat of combustion, 44

Corrugated paper boxes, heat of combustion, 66

Cotton thread, heat of combustion, 91

Cottonseed oil, hydrogenated, specific heat, 44

Cowpeas, heat of combustion, 37

Crude oil, heat of combustion, 62

Crude oil, isothermal compressibility, 62

Crude oil, specific heat, 63

Crustacean (see Animal)

Cyclotol, explosion temperature, 24

Cyclotol, heat of combustion, 23

Cyclotol, heat of explosion, 23

Cyclotol, heat of fusion, 24

Cyclotol, specific heat, 24

$D B X$, explosion temperature, 24

$D B X$, heat of combustion, 24

$D B X$, heat of explosion, 24

$D B X$, specific heat, 24

Deciduous shrubs, alpine tundra, heat of combustion, 72

Delrin, heat of combustion, 75 .

Dextrin, heat of combustion, 92

Diepoxide polymer, heat of combustion, 76

Dicyandiamide polymer, heat of combustion, 75

Distilled asphalt, Venezuelan, specific heat, 10

[juglas fir, specific heat, 108

Dowtherm A, specific heat, 18

Durethan U20, heat of combustion, 75 
Edelkunstharz $B$, heat of combustion, 81

Egg albumin, heat of combustion, 38

Egg albumin, specific heat, 38

Egg yolk, heat of combustion, 38

Elm, white, specific heat, 108

English walnut meat, heat of combustion, 47

Enthalpy (see Heat)

Epilox EG-1, hardened, heat of combustion, 75

Epilox EG-1, unhardened, heat of combustion, 76

Evergreen shrubs, alpine tundra, heat of combustion, 72

Evergreen shrub cuttings, heat of combustion, 72

Explosion, heat of (see Heat of explosion)

Explosion temperature, Amatol, 19

Explosion temperature, Baronal, 20

Explosion temperature, black powder, 20

Explosion temperature, Cyclotol, 24

Explosion temperature, DBX, 24

Explosion temperature, $\mathrm{H}-6,25$

Explosion temperature, $H B X-1,26$

Explosion temperature, $H B X-3,27$

Explosion temperature, HEX-24, 27

Explosion temperature, HEX-48, 28

Explosion temperature, HTA-3, 28

Explosior temperature, Minol-2, 32

Explosion temperature, MOX-1, 29

Explosion temperature, MOX-2B, 29

Explosion temperature, MOX-3B, 30

Explosion temperature, MOX-4B, 30

Explosion temperature, MOX-6B, 31

Explosion temperature, Octol, 33

Explosion temperature, PB-RDX, 31

Explosion temperature, Pentolite, 34

Explosion temperature, polyvinyl nitrate, 34

Explosion temperature, powder, black, 20

Explosion temperature, RDX-TNT Composition B, 22

Explosion temperature, Torpex, 35

Explosion temperature, Tritona 1,35

Explosive(s), 19

Fat, animal, heat of combustion, 38

Fat, animal, bovine, heat of combustion, 5,39

Fat, animal. 1ard, heat of combustion, 39

Fat, barley, heat of combustion, 38

Fat, beef, heat of combustion, 5,39

Fat, butter, heat of combustion, 39

Fat, fried, heat of combustion, 39

Fat, lard, heat of combustion, 39

Fat, mutton, heat of combustion, 40

Fat, pork, heat of combustion, 40

Fat, sheep, heat of combustion, 40

Fat, vegetable, heat of combustion, 38-41

Fat, wool, heat of combustion, 36

Fir, Douglas, specific heat, 108

Fir, heat of combustion, 108

Fish, guppie, heat of combustion, 7

Flatworm, heat of combustion, 6

Flea, water, heat of combustion, 5

Flour, heat of explosion, 42

Flour, soybean, heat of combustion, 41

Flour, specific heat, 42

Flour, wheat, heat of combustion, 42

Flower garden flants, heat of combustion, 71

Fly, caddis, heat of combustion, 6,7

Food(s), 36 
Food cartons, heat of combustion, 66

Food wastes, vegetable, heat of combustion, 42

Formaldehyde-melamine polymer, heat of combustion, 76

Formaldehyde-phenol resin, heat of combustion, 81

Formaldehyde-urea resin, heat of combustion, 89

Fruit, citrus, rinds and seeds, heat of combustion, 37

Fuel, aviation, heat of combustion, 48

Fuel, gasoline, auto-ignition temperature, 48

Fuel, jet, heat of combustion, 48

Fuel, 011 , heat of combustion, 63

Fusion, heat of (see Heat of fusion)

Garbage (see Refuse)

Gas, natura 1, compressibility, 49

Gas, natural, heat of combustion, 49

Gas oil, heat of combustion, 63

Gasoline, auto-ignition temperature, 48

Gasoline, aviation, heat of combustion, 48

Gelatine, heat of combustion, 95

Gelatine, blasting, heat of combustion, 21

Glass, Pyrex, heat content, 50

Glass, Pyrex, specific heat, 50

Glass, soda-1 ime, heat content, 51

Glass, soda-7ime, specific heat, 51

Glass temperature, Apiezon $T$ grease, 55

Glass transition temperature, Nylon-6, 78

Glass, Vycor, specific heat, 52

Gliadin, heat of combustion, 96

Gluten, heat of combustion, 96

Glutenin, heat of combustion, 97

Glycogen, heat of combustion, 92

Graphite, heat of combustion, 52

Grass, lawn clippings, heat of combustion, 70

Grease, Apiezon N, specific heat, 53

Grease, Apiezon P, heat of vaporization, 54

Grease, Apiezon P, vapor pressure, 54

Grease, Apiezon R, heat of vaporization, 54

Grease, Apiezon R, vapor pressure, 54

Grease, Apiezon S, heat of vaporization, 54

Grease, Apiezon S, vapor pressure, 54

Grease, Apiezon T, specific heat, 55

Green hydra, heat of combustion, 8

Gum, black, (tree) specific heat, 109

Gun cotton, specific heat, 21

Guppie, heat of combustion, 7

$H-6$, explosion temperature, 25

$\mathrm{H}-6$, heat of combustion, 25

$\mathrm{H}-6$, heat of explosion, 25

$\mathrm{H}-6$, heat of fusion, 25

$H-6$, specific heat, 25

Hastelloy $X$, heat content, 1

Hastelloy $x$, specific heat, 1

HBX-1, explosion temperature, 26

$H B X-1$, heat of combustion, 26

HBX-1, heat of explosion, 26

$H B X-1$, heat of fusion, 26

HBX-1, specific heat, 26

$H B X-3$, explosion temperature, 27

HBX -3 , heat of combustion, 27

HBX-3, heat of explosion, 27

HBX -3 , heat of fusion, 27

HBX-3, specific heat, 27 


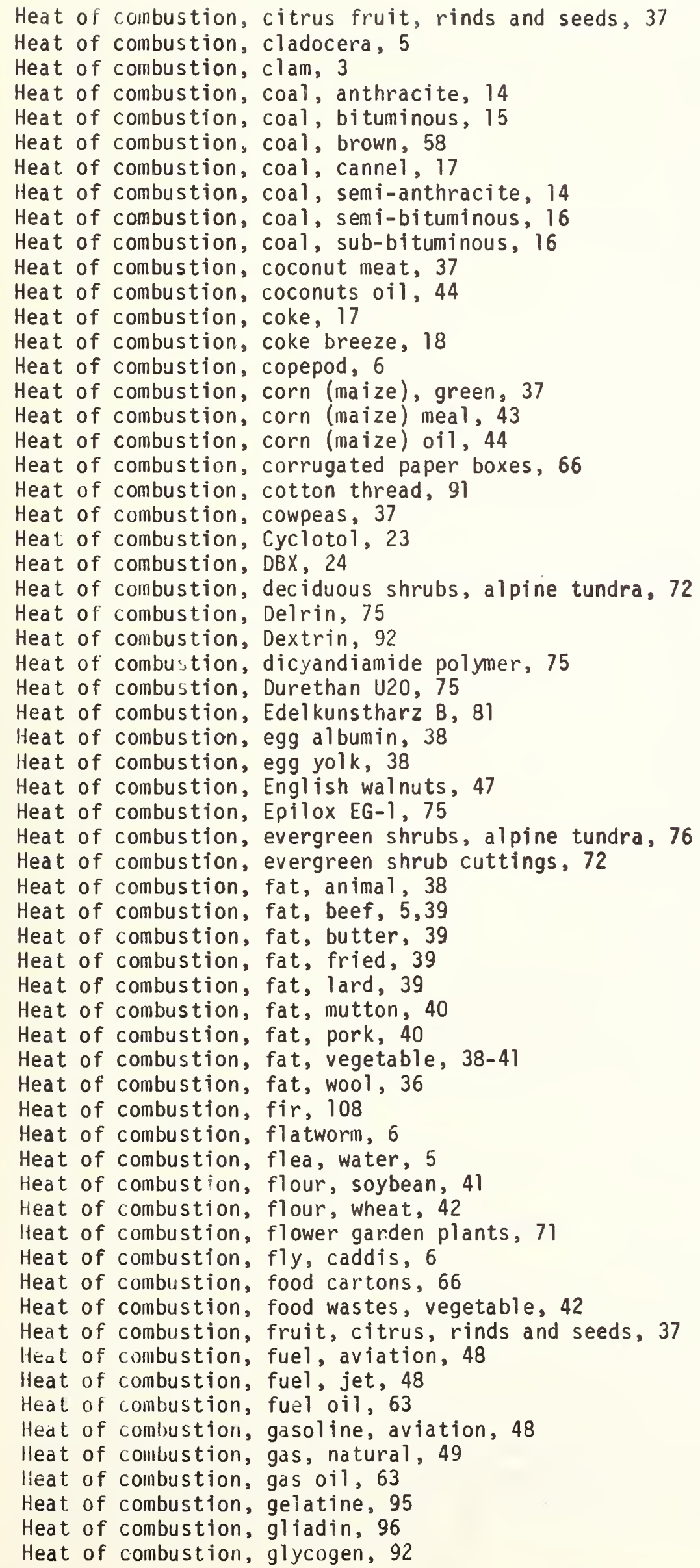


Heat of combustion, gluten, 96

Heat of combustion, glutenin, 97

Heat of combustion, graphite, 52

Heat of combustion, grass, lawn clippings, 70

Heat of combustion, guppie, 7

Heat of combustion, green hydra, 8

Heat of combustion, $\mathrm{H}-6,25$

Heat of combustion, HBX-1, 26

Heat of combustion, $H B X-3,27$

Heat of combustion, hemlock, 109

Heat of combustion, herbs, alpine tundra, 70

Heat of combustion, HEX-24, 27

Heat of combustion, HEX-48, 28

Heat of combustion, hickory, 109

Heat of combustion, hide, sheep, 7 .

Heat of combustion, hominy, 43

Heat of combustion, hornbeam, 109

Heat of combustion, HTA-3, 28

Heat of combustion, hydra, 7

Heat of combustion, inulin, 92

Heat of combustion, inulin triacetate, 92

Heat of combustion, isobutene polysulfone, 75

Heat of combustion, jet fuel, 48

Heat of combustion, jet naphtha, 60

Heat of combustion, JP-3, 48

Heat of combustion, JP- 4,48

Heat of combustion, JP-5, 48

Heat of combustion, kerosine, 49

Heat of combustion, lanolin, 36

Heat of combustion, lard, 39

Heat of combustion, leaves, tree, 70

Heat of combustion, Lexan, 84

Heat of combustion, lignin, 57

Heat of combustion, lignite, 58

Heat of combustion, magazine paper, 67

Heat of combustion, Makrolon, 84

Heat of combustion, manure, pig, 59

Heat of combustion, maple, 110

Heat of combustion, Marlex, 86

Heat of combustion, meal, corn, 43

Heat of combustion, meat, cooked scraps, 43

Heat of combustion, melamine-formaldehyde polymer, 76

Heat of combustion, 2,5-methylolmelamine, 76

Heat of combustion, 2-methylpropene polysulfone, 75

Heat of combustion, milk cartons, waxed, 68

Heat of combustion, milk casein, 94

Heat of combustion, Minol-2, 32

Heat of combustion, Miramid H, 78

Heat of combustion, Mirathen H, 86

Heat of combustion, mite, 8

Heat of combustion, mosses, alpine tundra, 71

Heat of combustion, MOX-1, 29

Heat of combustion, MOX-2B, 29

Heat of combustion, MOX-3B, 30

Heat of combustion, MOX-4B, 30

Heat of combustion, MOX-6B, 31

leat of combustion, municipal refuse, 98

Heat of combustion, mutton fat, 40

Heat of combustion, naphtha, Alaska, 59

Heat of combustion, naphtha, aromatie, 59

Heat of combustion, naphtha, high boiling, 60

Heat of combustion, naphtha, jet, 60

Heat of combustion, naphtha, low boiling, 60 
Heat of combustion, natural gas, 49

Heat of combustion, navy beans, 36

Heat of combustion, newsprint, 67

Heat of combustion, nitrocellulose, 21

Heat of combustion, Norway spruce, 114

Heat of combustion, Nylon-6,78

Heat of combustion, Nylon-6,6,80

Heat of combustion, oak, 110

Heat of combustion, oat 0il, 44

Heat of combustion, oats, rolled, 43

Heat of combustion, Octol, 32

Heat of combustion, oil, barley, 43

Heat of combustion, oil, coconut, 44

Heat of combustion, oil, corn (maize), 44

Heat of combustion, oil, crude, 62

Heat of combustion, oil, fuel, 63

Heat of combustion, oil, gas, 63

Heat of combustion, oil, oat, 44

Heat of combustion, oil, olive, 45

Heat of combustion, oil, paraffin, 65

Heat of combustion, oil, rye, 45

Heat of combustion, oil, wheat, 46

Heat of combustion, olive $0 i 1,45$

Heat of combustion, Oppanol B 100, 53

Heat of combustion, oyster, 3

Heat of combustion, paper, brown, 66

Heat of combustion, paper, corrugated box, 66

Heat of combustion, paper, food cartons, 66

Heat of combustion, paper, junk mail, 67

Heat of combustion, paper, magazine, 67

Heat of combustion, paper, newsprint, 67

Heat of combustion, paper, plastic coated, 68

Heat of combustion, paraffin 0i1, 105

Heat of combustion, paraffin wax, 105

Heat of combustion, PB-RDX, 31

Heat of combustion, peanuts, 46

Heat of combustion, pecans, 46

Heat of combustion, peat, 69

Heat of combustion, Pentolite, 34

Heat of combustion, petroleum jelly, 105

Heat of combustion, phenylalanine polypeptide, 80

Heat of combustion, phenol-formaldehyde resin, 81

Heat of combustion, pine, Ponderosa, 112

Heat of combustion, pine, white, 112

Heat of combustion, keratin, 97

Heat of combustion, plants, vascular aquatic, 71

Heat of combustion, plastic-coated paper, 68

Heat of combustion, polyacenaphthylene, 81

Heat of combustion, polyallyl phthalate, 81

Heat of combustion, poly-11-aminodecanoic acid, 88

Heat of combustion, poly-1-butene, 83

Heat of combustion, poly-1,1-dichloroethylene, 84

Heat of combustion, poly-1,1-difluoroethylene, 84

Heat of combustion, poljcaprolactam, 78

Heat of collibustion, polycarbonate, 84

Heat of combustion, poly-2,6-dimethylphenylene ether, 84

Heat of colibustion, polydioxydiphenyl propane carbonate, 84

lleat of combustion, polyethylene, 85

Heat of combustion, poly-z-enantholactam, 85

Heat of combustion, polyhexamethylene adipamide, 80

lieat of combustion, polymethylmethacrylate, 76

Heat of combustion, Polyharnstoff, 88

Heat of combustion, polyisobutene, 83 
Heat of combustion, polyisobutylene, 83

Heat of combustion, poly-N-ethyl-N-methylpropionamide, 90

Heat of combustion, polymethyls tyrene, 86

Heat of combustion, polyoxymethylene, 75

Heat of combustion, polypeptide, poly-N-ethyl-N-methylpropionamide, 89

Heat of combustion, polypeptide, phenylalanine, 90

Heat of combustion, polypeptide, sarcosine, 90

Heat of combustion, polypropylene, 87

Heat of combustion, polystyrene, 87

Heat of combustion, polysulfones, 74

Heat of combustion, polytetrafluoroethylene, 89

Heat of combustion, polyurea, 88

Heat of combustion, polyurethane, 75

Heat of combustion, polyvinyl nitrate, 34

Heat of combustion, polyvinylidene chloride, 84

Heat of combustion, polyvinylidene fluoride, 84

Heat of combustion, Ponderosa pine, 112

Heat of combustion, poplar, 112

Heat of combustion, pork fat, 40

Heat of combustion, potato, white, 46

Heat of combustion, prolomin, 96

Heat of combustion, propellant, (OS/AP), 93

Heat of combustion, propellant, (PS/AP), 94

Heat of combustion, propene, 2-methyl, polysulfone, 75

Heat of combustion, protein, bovine, 5

Heat of combustion, protein, wool, 97

Heat of combustion, RDX-TNT Composition B, 22

Heat of combustion, refuse, brown paper, 66

Heat of combustion, refuse, citrus fruit, rinds and seeds, 37

Heat of combustion, refuse, corrugated paper boxes, 66

Heat of combustion, refuse, evergreen shrub cuttings, 72

Heat of combustion, refuse, fat, fried, 39

Heat of combustion, refuse, flower garden plants, 71

Heat of combustion, refuse, food cartons, 66

Heat of combustion, refuse, grass, lawn clippings, 70

Heat of combustion, refuse, junk mai1, 67

Heat of combustion, refuse, magazine paper, 67

Heat of combustion, refuse, meat, cooked scraps, 43

Heat of combustion, refuse, municipal, 98

Heat of combustion, refuse, newsprint, 67

Heat of combustion, refuse, paper, brown, 66

Heat of combustion, refuse, paper, corrugated box, 66

Heat of combustion, refuse, paper, food cartons, 66

Heat of combustion, refuse, paper, junk mail, 67

Heat of combustion, refuse, paper, magazine, 67

Heat of combustion, refuse, paper, plastic coated, 68

Heat of combustion, refuse, paper, waxed milk cartons, 68

Heat of combustion, refuse, plastic coated paper, 68

Heat of combustion, refuse, shoe, 101

Heat of combustion, refuse, tree leaves, 70

Heat of combustion, refuse, vacuum cleaner catch, 105

Heat of combustion, refuse, vegetable food wastes, 42

Heat of combustion, refuse, waxed milk cartons, 68

lleat of combustion, resin, phenol-formaldehyde, 81

lleat of combustion, rice, 47

Heat of combustion, Rilsan, 88

Heat. of combustion, rolled oats, 43

Hent. of combustion, rutber, 99

ileat of combustion, rubber-sulfur compounds, 99

lleal of combusiton, rye oil, 45

Heat of combustion, sarcosine polypeptide, 90

lleat of combustion, semi-anthracite coal, 14

lleat of combustion, semi-bituminous coal, 16 


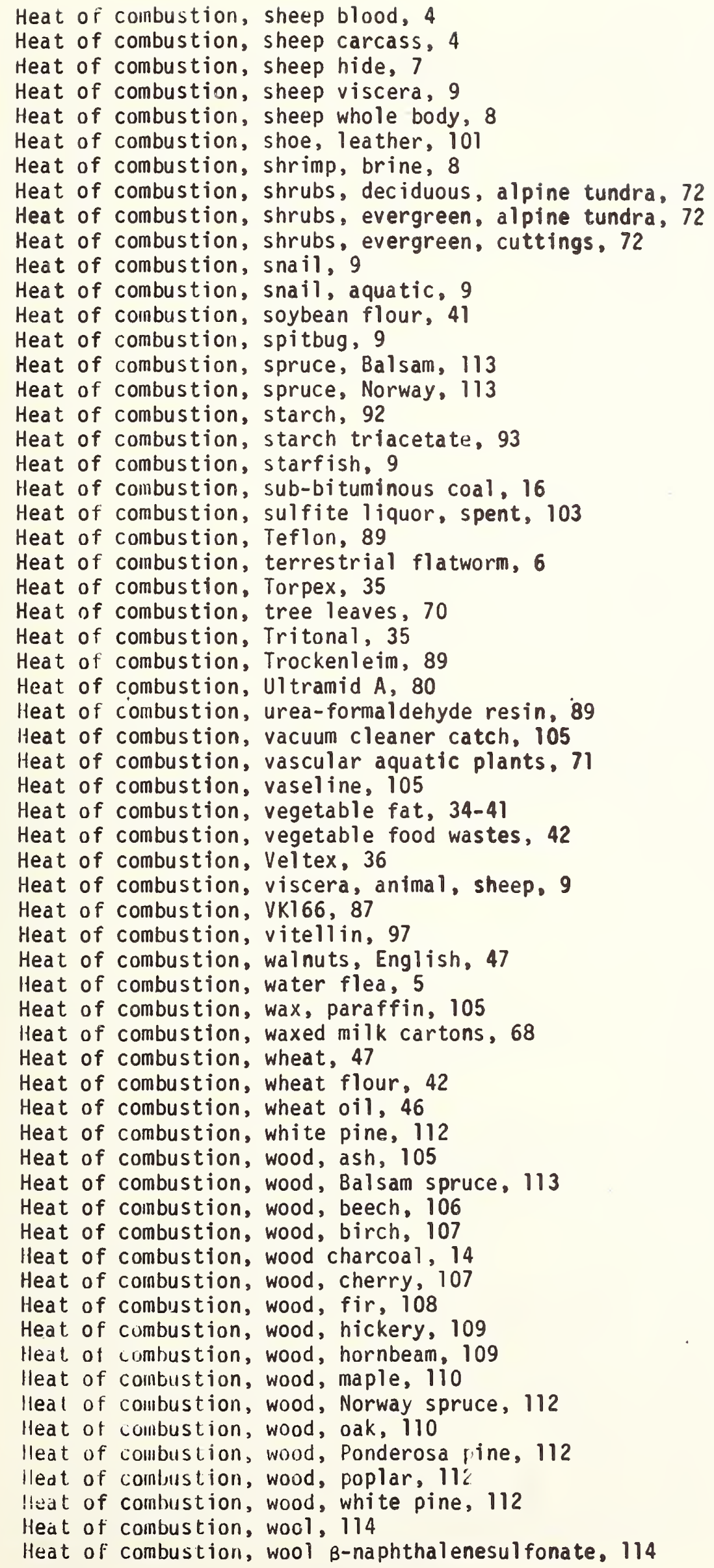




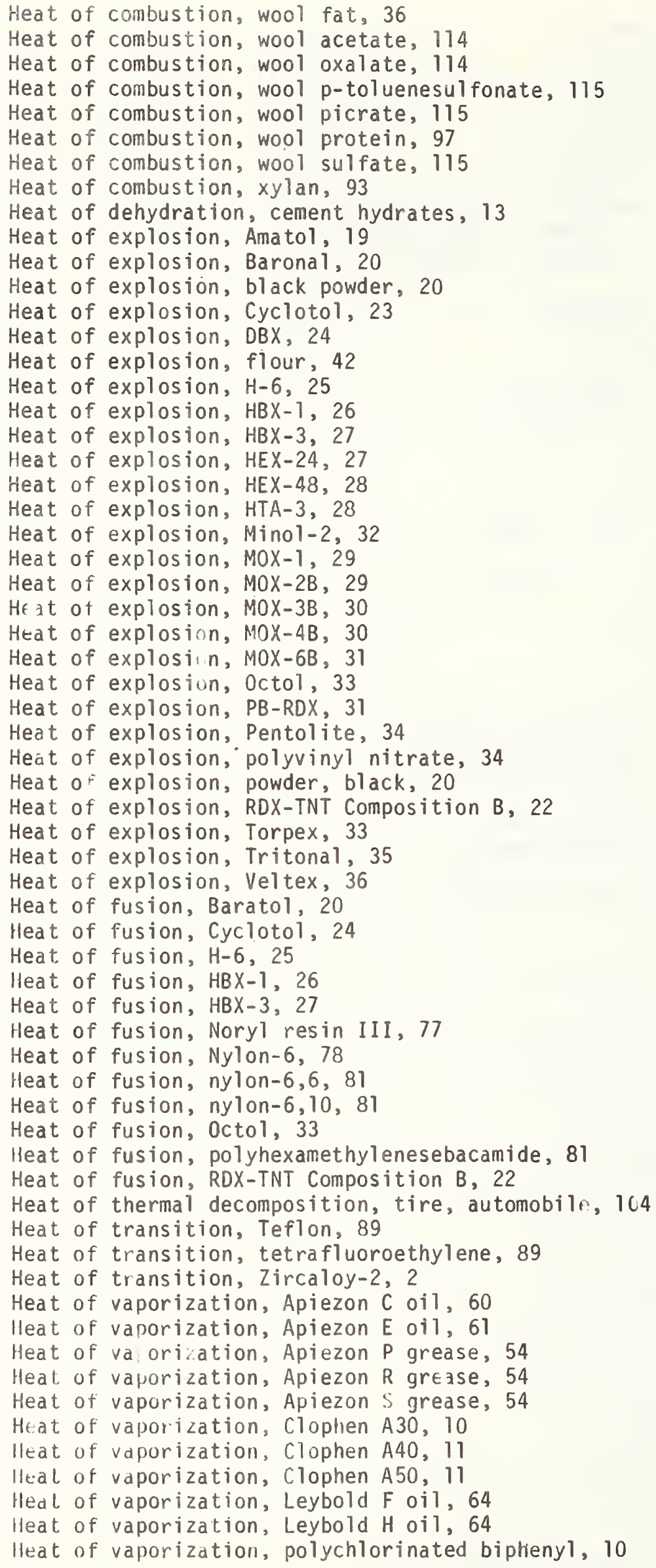


Hemlock, western, specific heat, 109

Herbs, alpine tundra, heat of combustion, 70

HEX-24, explosion temperature, 27

HEX-24, heat of combustion, 27

HEX-24, heat of explosion, 27

HEX-48, explosion temperature, 28

HEX-48, heat of combustion, 28

HEX-48, heat of explosion, 28

Hickory, heat of combustion, 109

Hide, sheep, heat of combustion, 7

Holocellulose, specific heat, 91

Hominy, heat of combustion, 43

Hornbeam, heat of combustion, 109

HTA-3, explosion temperature, 28

HTA-3, heat of combustion, 28

HTA-3, heat of explosion, 28

HTA-3, specific heat, 28

Hydra, heat of combustion, 7

Hydra, green, heat of combustion, 8

Insect(s) (see Animal(s))

Insulin, bovine zinc, specific heat, 55

Inulin, heat of combustion, 92

Inul in triacetate, heat of combustion, 92

Iron, cast, heat content, 56

Isobutene polysulfone, heat of combustion, 75

Jet fuels, heat of combustion, 18

Jet naphtha, heat of combustion, 60

$J P-3$, heat of combustion, 48

$J P-4$, heat of combustion, 48

$J P-5$, heat of combustion, 48

Junk mail, heat of combustion, 67

Kerotin, heat of combustion, 97

Kerosine, heat of combustion, 49

Lanol in, heat of combustion, 36

Lard, heat of combustion, 39

Lawn grass clippings, heat of combustion, 70

Leather, specific heat, 56

Leaves, tree, heat of combustion, 70

Lexan, heat of combustion, 84

Leybold $F$ oil, heat of vaporization, 64

Leybold $F$ oil, vapor pressure, 64

Leybold $H$ oil, heat of vaporization, 64

Leybold $H$ oil, vapor pressure, 64

Lignin, heat of combustion, 57

Lignin, specific heat, 57

Lignite, heat of combustion, 58

Linseed oil, specific heat, 64

loblolly pine, extractives, specific heat, 111

Loblolly pine, specific heat, 111

Longleaf pine, specific heat, 111

lubricating oil, vapor pressure, 64

Lucite, specific heat, 76

Lunar material, specific heat, 58,59

Magazine paper, heat of combustion, 67

Ma clampah (tree), specific heat, 110

Maize (see Corn)

Makrolon, heat of cornbustion, 84

Manure, pig, heat of combustion, 59 
Maple, heat of combustion, 110

Maple, sugar (tree), specific heat, 110

Marlex, heat of combustion, 86

Meal, corn (maize), heat of combustion, 43

Meat, cooked scraps, heat of combustion, 43

Melamine-formaldehyde polymer, heat of combustion, 76

2,5-Methylolmelamine, heat of combustion, 76

2-methylpropene polysulfone, heat of combustion, 75

Milk casein, heat of combustion, 94

Milk cartons, waxed, heat of combustion, 68

Minol-2, explosion temperature, 32

Minol-2, heat of combustion, 32

Minol-2, heat of explosion, 32

Minol-2, specific heat, 32

Miramid $\mathrm{H}$, heat of combustion, 78

Mirathen $H$, heat of combustion, 86

Mite, heat of combustion, 8

Mollusk (see Animal(s))

Mosses, alpine tundra, heat of combustion, 71

MOX-1, explosion temperature, 29

MOX-1, heat of combustion, 29

MOX-1, heat of explosion, 29

MOX-2B, explosion temperature, 29

MOX-2B, heat of combustion, 29

MOX-2B, heat of explosion, 29

MOX-3B, explosion temperature, 30

MOX-3B, heat of combustion, 30

$M O X-3 B$, heat of explosion, 30

$M O X-4 B$, explosion temperature, 30

MOX-4B, heat of combustion, 30

MOX-4B, heat of explosion, 30

MOX-6B, explosion temperature, 31

MOX-6B, heat of combustion, 31

MOX-6B, heat of explosion, 31

Municipal refuse, heat of combustion, 98

Mutton fat, heat of combustion, 40

Naphitha, Alaska, heat of combustion, 59

Naphtha, aromatic, heat of combustion, 59

Naphtha, high boiling, heat of combustion, 60

Naphtha, jet, heat of combustion, 60

Naphtha, low boiling, heat of combustion, 60

Natural gas, compressibility, 49

Natural gas, heat of combustion, 49

Navy beans, heat of combustion, 36

Neatsfoot oil, specific heat, 65

Newsprint, heat of combustion, 67

Nickel-chromium alloy, specific heat, 1

Nitrocellulose, heat of combustion, 21

Nitrocellulose, specific heat, 21

Noryl resin III, heat of fusion, 77

Noryl resin III, specific heat, 77

Norway spruce, heat of combustion, 113

Nylon-6, glass transition temperature, 78

Nylon-6, heat of fusion, 78

Nylon-6, heat of combustion, 78

Nylon-6, specific heat, 78

Nylun-6,6, heat of combustion, 80

Nylon-6,6, heat of fusion, 81

W, lon-6,6, specific heat, 80

Nylon-6,10, heat of fusion. 81

Nylon-6,10, specific heat, 81 
Oak, heat of combustion, 110

Oak, red, specific heat, 111

Oak, white, specific heat, 111

Oat oil, heat of combustion, 44

0ats, rolled, heat of combustion, 43

Octol, explosion temperature, 33

Octol, heat of combustion, 32

Octol, heat of explosion, 33

Octol, heat of fusion, 33

Octol, specific heat, 33

011 . Apiezon $C$, heat of vaporization, 60

$0 i 1$, Apiezon $C$, vapor pressure, 60

$0 i 1$. Apiezon $E$, heat of vaporization, 61

$0 i 1$. Apiezon $E$, vapor pressure, 61

$0 i 1$, barley, heat of combustion, 43

$0 i 1$, castor, specific heat, 61

$0 i 1$, coconut, heat of combustion, 44

$0 i 1$, corn (maize), heat of combustion, 44

$0 i 1$, cottonseed, hydrogenated, specific heat, 44

$0 i 1$, crude, heat of combustion, 62

$0 i 1$, crude, isothermal compressibility, 62

$0 i 1$, crude, specific heat, 63

$0 i 1$, fuel, heat of combustion, 63

$0 i 1$, gas, heat of combustion, 63

$0 i 1$, Leybold $F$, heat of vaporization, 64

$0 i 1$, Leybold $F$, vapor pressure, 64

$0 i 1$, Leybold $H$, heat of vaporization, 64

$0 i 1$, Leybold $H$, vapor pressure, 64

$0 i 1,1$ inseed, specific heat, 64

0il, lubricating, vapor pressure, 64

$0 i 1$, neatsfoot, specific heat, 65

$0 i 1$, oat, heat of combustion, 44

011, olive, heat of combustion, 45

$0 i 1$, ol ive, vapor pressure, 45

$0 i 1$, paraffin, heat of combustion, 65

$0 i 1$, perilla, specific heat, 65

$0 i 1$, rye, heat of combustion, 45

$0 i 1$, soybean, specific heat, 45

$0 i 1$, soybean, vapor pressure, 45

$0 i 1$, tung, specific heat, 65

$0 i 1$, wheat, heat of combustion, 46

01 ive oil, heat of combustion, 45

01 ive oil, vapor pressure, 45

Oppanol B100, heat of combustion, 83

DS/AP propellant, heat of combustion, 93

Oyster, heat of combustion, 3

Paper, brown, heat of combustion, 66

Paper, corrugated box, heat of combustion, 66

Paper, food cartons, heat of combustion, 66

Paper, junk inail, heat of combustion, 67

Paper, magazine, heat of combustion, 67

Paper, milk cartons, waxed, heat of combustion, 68

Paper, newsprint, heat of combustion, 67

Paper, plastic coated, heat of combustion, 68

Paper, waxed milk cartons, heat of combustion, 68

Paraffin oil, heat of combustion, 65

Paraffin wax, heat of combustion, 105

PB-RDX, explosion temperature, 31

PB-RDX, heat of combustion, 31

PB-RDX, heat of explosion, 31

Peanuts, heat of combustion, 46

Peat, heat of combustion, 69 
Pecans, heat of combustion, 46

Pentolite, explosion temperature, 34

Pentolite, heat of combustion, 34

Pentolite, heat of explosion, 34

Perilla oil, specific heat, 65

Petroleum jelly, heat of combustion, 105

Phenol-formaldehyde resin, heat of combustion, 81

Phenylalanine polypeptide, heat of combustion, 90

Pig manure, heat of combustion, 59

Pine, Loblolly, extractives, specific heat, 111

Pine, Loblolly, wood, specific heat, 111

Pine, longleaf, specific heat, 111

Pine, Ponderosa, heat of combustion, 112

Pine, white, heat of combustion, 112

PKU T 22, heat of combustion, 84

Plastic coated paper, heat of combustion, 68

Plants, 69

Plants, vascular aquatic, heat of combustion, 71

Plastic(s) (see Polymer(s))

Plastic, celluloid, heat of combustion, 13

Polyacenaphthylene, heat of combustion, 81

Poly-11-aminodecanoic acid, heat of combustion, 88

Polyallylphthalate, heat of combustion, 81

Polybutadiene, specific heat, 81

Polybutadiene-acrylonitrile-styrene copolymer, specific heat, 88

Poly-1-butene, heat of combustion, 83

Polybutylene, heat of combustion, 83

Polycaprolactam, heat of combustion, 78

Polycarbonate, heat of combustion, 84

Polychlorinated biphenyl, Clophen $\mathrm{A} 30$, heat of vaporization, 10

Polychlorinated biphenyl, Clophen $\mathrm{A} 30$, vapor pressure, 10

Polychlorinated biphenyl, Clophen $A 40$, heat of vaporization, 11

Polychlorinated biphenyl, Clophen $A 40$, vapor pressure, 11

Polychlorinated biphenyl, Clophen $\mathrm{A} 50$, heat of vaporization, 11

Polychlorinated biphenyl, Clophen A50, vapor pressure, 11

Poly-1,1-dichloroethylene, heat of combustion, 84

Poly-1,1-difluoroethylene, heat of combustion, 84

Poly-2,6-dimethylphenylene ether, heat of combustion, 84

Polydioxydiphenyl propane carbonate, heat of combustion, 84

Poly-e-enantholactam, heat of combustion, 85

Polyethylene, heat of combustion, 85

Polyethylene, specific heat, 85

Polyharnstoff, heat of combustion, 88

Polyhexamethyleneadipamide, heat of combustion, 80

Polyhexamethyleneadipamide, heat of fusion, 81

Polyhexamethyl eneadipamide, specific heat, 80

Polyhexamethylenesebacamide, heat of fusion, 81

Polyhexamethylenesebacamide, specific heat, 81

Polyisobutene, heat of combustion, 83

Polyisobutylene, heat of combustion, 83

Polymer(s), 73

Polymethylmethacrylate, heat of combustion, 76

Polymethylmethacrylate, specific heat, 76

Polymethylstyrene, heat of combustion, 86

Poly-N-ethyl-N-methylpropionamide, heat of combustion, 90

Polyoxyrithylene, heat of combustion, 75

Polypeptide, phenylalanine, heat combustion, 90

Pulypeptide, sarcosine, heat of combustion, 90

Polypropylene VK 166, heat combustion, 87

Polyouccharide(s), 90

Polystyrene, heat of combustion, 87

Polystyrol, heat of combustion, 87

Polysilfone, butene, heat of combustion, 74 
Polytetrafluoroethylene, heat of combustion, 89

Polytetrafluoroethylene, specific heat, 89

Polyurea, heat of combustion, 88

Polyurethane, heat of combustion, 75

Polyvinylidene chloride, heat of combustion, 84

Polyvinylidene fluoride, heat of combustion, 84

Polyvinyl nitrate, explosion temperature, 34

Polyvinyl nitrate, heat of combustion, 34

Polyvinyl nitrate, heat of explosion, 34

Ponderosa pine, heat of combustion, 112

Poplar, heat of combustion, 112

Porcelain, specific heat, 93

Pork fat, heat of combustion, 40

Powder, black, heat of explosion, 20

Potato, white, heat of combustion, 46

Potato, white, specific heat, 46

Prolomin, heat of combustion, 96

Propellant OS/AP, heat of combustion, 93

Propellant PS/AP, heat of combustion; 93

Propellant $x 14$, specific heat, 94

Propene 2-methylpolysulfone, heat of combustion, 75

Protein, animal, bovine, heat of combustion, 5

Protein, collagen, specific heat, 95

Protein, wool, heat of combustion, 97

Protozoa (see Animal(s))

PS/AP propellant, heat of combustion, 94

Pyroxylin, specific heat, 21

Pyrex glass, heat content, 50

Pyrex glass, specific heat, 50

Quaking aspen, specific heat, 106

Quebracho tannin extract, specific heat, 103

Red cedar, specific heat, 107

Red spruce, specific heat, 113

Refuse, boxes, corrugated paper, heat of combustion, 66

Refuse, brown paper, heat of combustion, 66

Refuse, citrus fruit, rinds and seeds, heat of combustion, 37

Refuse, corrugated paper boxes, heat of combustion, 66

Refuse, evergreen shrub cuttings, heat of combustion, 72

Refuse, fat, fried, heat of combustion, 39

Refuse, flower garden plants, heat of combustion, 71

Refuse, food cartons, heat of combustion, 66

Refuse, food wastes, vegetable, heat of combustion, 42

Refuse, junk mail, heat of combustion, 67

Refuse, magazine paper, heat of combustion, 67

Refuse, meat, cooked scraps, heat of combustion, 43

Refuse, municipal, heat of combustion, 98

Refuse, newsprint, heat of combustion, 67

Refuse, paper, brown, heat of combustion, 66

Refuse, paper, corrugated box, heat of combustion, 66

Refuse, paper, food cartons, heat of combustion, 66

Refuse, paper, junk mail, heat of combustion, 67

Refuse, paper, magazine, heat of combustion, 67

Refuse, paper, milk cartons, waxed, heat of combustion, 68

Refuse, paper, newsprint, heat of combustion, 67

Refuse, paper, plastic coated, heat of combustion, 68

Refuse, paper, waxed milk cartons, heat of combustion, 68

Refuse, plastic coated paper, heat of combustion, 68

Refuse, shoe, leather, heat of combustion, 101

Refuse, tree leaves, heat of combustion, 70

Refuse, vacuum cleaner catch, heat of combustion, 105

Resin, ABS I, specific heat, 88 
Resin, phenol-formaldehyde, heat of combustion, 81

Resin, urea-formaldehyde, heat of combustion, 89

Rice, heat of combustion, 47

Rolled oats, heat of combustion, 43

Rilsan, heat of combustion, 88

Rock, lunar, vesicular basalt, specific heat, 58

Rubber, heat of combustion, 99

Rubber-sulfur compounds, heat of combustion, 99

Rye 011 , heat of combustion, 45

Santowax R, specific heat, 99

Sarcosine polypepide, heat of combustion, 90

Sea water, specific heat, 100

Semi-anthracite coal, heat of combustion, 14

Semi-bituminous coal, heat of combustion, 16

Sheep blood, heat of combustion, 4

Sheep carcass, heat of combustion, 4

Sheep hide, heat of combustion, 7

Sheep viscera, heat of combustion, 10

Sheep, whole body, heat of combustion, 8

Shoe, leather, heat of combustion, 101

Shrimp, brine, heat of combustion, 8

Shrubs, deciduous, alpine tundra, heat of combustion, 72

Shrubs, evergreen, alpine tundra, heat of combustion, 72

Shrubs, evergreen cuttings, heat of combustion, 72

Silver A Zeolite, specific heat, 115

Snail, heat of combustion, 9

Snail, aquatic, heat of combustion, 9

Soda-lime glass, heat content, 51

Soda-lime glass, specific heat, 51

Sodium A Zeolite, specific heat, 115

Soil, regolith, lunar, specific heat, 59

Soybean flour, heat of combustion, 41

Soybean oil, specific heat, 45

Soybean oil, vapor pressure, 45

Specific heat, ABSI Resin, 88

Specific heat, alloy steel, NPL No. 19, 101

Specific heat, alloy, titanium, Ti-75A, 2

Specific heat, alloy, titanium, Ti-150A, 2

Specific heat, Amatol, 19

Specific heat, Apiezon $N$ grease, 53

Specific heat, Apiezon $T$ grease, 55

Specific heat, ash, white (wood), 106

Specific heat, aspen, quaking, 106

Specific heat, asphalt, 10

Specific heat, Baratol, 20

Specific heat, bark, spruce pine, 114

specific heat, basalt, lunar, 58

Specific heat, beech, 106

Specific heat, black gum (wood), 109

Specific heat, bovine chymotrypsinogen A, 94

Specific heat, bovine zinc insulin, 55

Specific heat, brass, 12

Specific heat, butadiene-styrene copolymer, GL-622, 73

Specific heat, butadiene-styrene copolymer, $X-454,73$

Specific heat, butadiene-styrene copolymer, X-478, 74

Specific heat, castor oil, 61

Specific heat, cedar, red, 107

Specific heat, $\alpha$-cellulose, 91

Specific heat, cellulose nitrate, 21

Specific heat, chestnut (wood), 108

Specific heat, chestnut tannin extract, 103 
Specific heat, chromium-nickel alloy, 1

Specific heat, Cocobola Negra, 108

specific heat, collagen, 98

Specific heat, cottonseed oil, hydrogenated, 44

Specific heat, crude oil, 63

Specific heat, Cyclotol, 24

Specific heat, DBX, 24

Specific heat, distilled asphalt, Venezuelan, 10

Specific heat, Douglas fir, 108

Specific heat, Dowtherm A, 18

Specific heat, egg albumin, 38

Specific heat, eim, white, 108

Specific heat, fir, Douglas, 108

Specific heat, flour, 42

Specific heat, glass, Pyrex, 50

Specific heat, glass, soda-lime, 51

Specific heat, glass, Vycor, 51

Specific heat, grease, Apiezon N, 53

Specific heat, grease, Apiezon T, 55

Specific heat, gum, black (wood), 109

Specific heat, gun cotton, 21

Specific heat, $H-6,25$

Specific heat, Hastelloy $x, 1$

Specific heat, $H B X-1,26$

Specific heat, $H B X-3,27$

Specific heat, hemlock, western, 109

Specific heat, holocellulose, 91

Specific heat, HTA-3, 28

Specific heat, insulin, bovine zinc, 55

Specific heat, leather, 56

Specific heat, lignin, 57

Specific heat, linseed oil, 64

Specific heat, loblolly pine extractives, 111

Specific heat, loblolly pine, 111

Specific heat, longleaf pine, 111

Specific heat, Lucite, 76

Specific heat, lunar material, 58

Specific heat, Mai champah (wood), 110

Specific heat, maple, sugar, 110

Specific heat, Minol-2, 32

Specific heat, neatsfoot oi1, 65

Specific heat, nickel-chromium alloy, 1

Specific heat, nitrocellulose, 21

Specific heat, Noryl resin III, 77

Specific heat, Nylon-6,78

Specific heat, Nylon-6,6,80

Specific heat, Nylon-6,10,81

Specific heat, oak, 111

Specific heat, Octol, 33

Specific heat, 011 , castor, 61

Specific heat, oil, cottonseed, hydrogenated, 44

Specific heat, oil, crude, 63

Specific heat, oil, linseed, 64

Specific heat, oil, neatsfoot, 65

Specific heat, oil, perilla, 65

Specific heat, oil, soybean, 45

Specific heat, oil, tung, 65

Specific heat, perilla oil, 65

Specific heat, pine, longleaf, 111

Specific heat, polybutadiene, 82

Specific heat, polyethylene, 85

Specific heat, polyhexamethylenesebacamide, 81

Specific heat, polyhexamethyleneadipamide, 80 
Specific heat, polymethylmethacrylate, 76

Specific heat, polytetrafluoroethylene, 89

Specific heat, porcelain, 93

Specific heat, potato, white, 46

Specific heat, propellant X-14, 94

Specific heat, Pyrex glass, 50

Specific heat, pyroxylin, 21

Specific heat, quaking aspen, 106

Specific heat, RDX-TNT composition B, 22

Specific heat, red cedar, 107

Specific heat, red oak, 111

Specific heat, red spruce, 113

Specific heat, Resin ABSI, 88

Specific heat, Santowax R, 99

Specific heat, sea water, 100

Specific heat, silver A zeolite, 115

Specific heat, soda-lime glass, 51

Specific heat, sodium A zeolite, 115

Specific heat, soil, lunar, 59

Specific heat, soybean oil, 45

Specific heat, spruce pine, 113

Specific heat, spruce pine bark, 114

Specffic heat, spruce, red, 113

Specific heat, stainless steel En-58C, 102

Specific heat, stainless steel 347,101

Specific heat, stainless steel 446, 102

Specific heat, steel, stainless En-58C, 102

Specific heat, steel, stainless, 347, 101

Specific heat, steel, stainless, 446, 102

Specific heat, styrene-acrylonitrile-butadiene copolymer, 88

Specific heat, sugar maple, 110

Specific heat, tannin extract, 103

Specific heat, tannin extract, chestnut, 103

Specific heat, Teflon, 89

Specific heat, titanium alloy, Ti-75A, 2

Specific heat, titanium alloy, Ti-150A, 2

Specific heat, topaz, 104

Specific heat, Torpex, 35

Specific heat, Tritonal, 35

Specific heat, tung oil, 65

Specific heat, Vycor glass, 51

Specific heat, western hemlock, 109

Specific heat, white elm, 108

Specific heat, white oak, 111

Specific heat, wood, ash, 106

Specific heat, wood, beech, 106

specific heat, wood, black gum, 109

Specific heat, wood, chestnut, 108

Specific heat, wood, cocobola negra, 108

Specific heat, wood, Douglas fir, 108

Specific heat, wood, loblolly pine, 111

Specific heat, wood, longleaf pine, 111

Specific heat, wood, Mai champah, 110

Specific heat, wood, oak, 111

Specific heat, wood, red cedar, 107

Specific heat, wood, red spruce, 113

specific heat, wood, quaking aspen, 106

specific heat, wood, spruce pine, 113

specific heat, wood, sugar maple, 110

specific heat, wood, western hemlock, 109

specific heat, wood, white elm, 108

Specific heat, $x 14$ propellant, 94

Specific heat, zeolite, silver A, 115 
Specific heat, zeolite, sodium A, 115

Specific heat, Zircaloy-2, 2

Spitbug, heat of combustion, 9

Spruce, balsam, heat of combustion, 113

Spruce, Norway, heat of combustion, 113

Spruce pine, specific heat, 113

Spruce pine bark, specific heat, 114

Spruce, red, specific heat, 113

Stainless steel En-58C, specific heat, 102

Stainless steel 347 , specific heat, 101

Stainless steel 446, specific heat, 102

Starch, heat of combustion, 92

Starch triacetate, heat of combustion, 93

Starfish, heat of combustion, 9

Steel, alloy, NPL No 19, specific heat, 101

Steel, stainless En-58C, specific heat, 102

Steel, stainless, 347 , specific heat, 101

Steel, stainless, 446 , specific heat, 102

Styrene-acrylonitrile-polybutadiene copolymer, specific heat, 88

Styrene-butadiene copolymer, $X-454$, specific heat, 73

Styrene-butadiene copolymer, $X-478$, specific heat, 74

Styrene-butadiene copolymer, GL-622, specific heat, 73

Sub-bituminous coal, heat of combustion, 16

Suet (see fat, beef, and fat, mutton)

Sugar maple, specific heat, 110

Sulfite liquor, spent, heat of combustion, 103

Tannin extract, specific heat, 103

Tannin extract, chestnut, specific heat, 103

Teflon, heat of combustion, 89

Teflon, heat of transition, 89

Teflon, specific heat, 89

Terrestrial flatworm, heat of combustion, 6

Tetrafluoroethylene, heat of transition, 89

Ti-75A, titanium alloy, specific heat, 2

Ti-150A, titanium alloy, specific heat, 2

Tire, automobile, heat of thermal decomposition, 104

Titanium alloy Ti-75A, specific heat, 2

Titanium alloy, Ti-150A, specific heat, 2

Topaz, specific heat, 104

Torpex, explosion temperature, 35

Torpex, heat of combustion, 35

Torpex, heat of explosion, 35

Torpex, specific heat, 35

Transition, heat of (see Heat of transition)

Trash, municipal (see Refuse)

Tree(s) (see Wood)

Tree leaves, heat of combustion, 70

Tritonal, explosion temperature, 35

Tritonal, heat of combustion, 35

Tritonal, heat of explosion, 35

Tritonal, specific heat, 35

Trockenleim, heat of combustion, 89

Tung oil, specific heat, 65

Ultramid $A$, heat of combustion, 80

Urea-furisialdehyde resin, heat of combustion, 89

Vacium cleaner catch, heat of combustion, 105

Vapor pressure, Apiezon C oil, 60

Vapor pressure, Apiezon E 011,61

Vapor pressure, Apiezon P grease, 54

Vapor pressure, Apiezon $R$ grease, 54 
Vapor pressure, Apiezon S grease, 54

Vapor pressure, Clophen A30, 10

Vapor pressure, Clophen $A 40,11$

Vapor pressure, Clophen A50, 11

Vapor pressure, Leybold F oil, 64

Vapor pressure, Leybold $\mathrm{H}$ oil, 64

Vapor pressure, lubricating oil, 64

Vapor pressure, oil, lubricating, 64

Vapor pressure, oil, olive, 45

Vapor pressure, oil, soybean, 45

Vapor pressure, olive oil, 45

Vapor pressure, polychlorinated biphenyl, 10

Vapor pressure, soybean oil, 45

Vaporization, heat of (see Heat of vaporization)

Vascular aquatic plants, heat of combustion, 71

Vaseline, heat of combustion, 105

Vegetable fat, heat of combustion, 38-41

Vegetable food wastes, heat of combustion, 42

Veltex, heat of combustion, 36

Veltex, heat of explosion, 36

Venezuelan distilled asphalt, specific heat, 10

Viscera, sheep, heat of combustion, 10

Vitellin, heat of combustion, 97

VK166 polypropylene, heat of combustion, 87

vycor glass, specific heat, 51

Walnuts, English, heat of combustion, 47

Water, sea, specific heat, 100

Water fleas, heat of combustion, 5

Wax, paraffin, heat of combustion, 105

Waxed milk cartons, heat of combustion, 68

Western hemlock, specific heat, 109

Wheat, heat of combustion, 47

Wheat flour, heat of combustion, 42

Wheat oil, heat of combustion, 46

White oak, specific heat, 111

White elm, specific heat, 108

White pine, heat of combustion, 112

Wood, ash, heat of combustion, 105

Wood, ash, white, specific heat, 106

Wood, aspen, quaking, specific heat, 106

Wood, beech, heat of combustion, 106

wood, beech, specific heat, 106

Wood, birch, heat of combustion, 107

Wood, black gum, specific heat, 109

Wood, cedar, red, specific heat, 107

Hood charcoal, heat of combustion, 14

Wood, cherry, heat of combustion, 107

Wood, chestnut, specific heat, 108

Wood, Cocobola negra, specific heat, 108

Wood, Douglas fir, specific heat, 108

Wood, elm, white, specific heat, 108

Wood, fir, heat of combustion, 108

Wood, fir, Douglas, specific heat, 108

Wood, gurn, black, specific heat, 109

Wood, liemlock, western, specific heat, 109

Wood, hickory, heat of combustion, 109

Wood, hulnbeam, heat of combustion, 109

Wood, loblolly pine, specific heat, 111

Wool, longled pine, specific heat, 111

Hood, lla i champah, specific heat, 110

Woud, Ilaple, heat of cumbustion, 110

Woul, Imaple, sugal, specific heat, 110 
Wood, oak, heat of combustion, 110

Wood, oak, specific heat, 110

Wood, pine, loblolly, specific heat, 111

Wood, pine, longleaf, specific heat, 111

Wood, pine, Ponderosa, heat of combustion, 112

Wood, Ponderosa pine, heat of combustion, 112

Wood, poplar, heat of combustion, 112

Wood, quaking aspen, specific heat, 106

Wood, red cedar, specific heat, 107

Wood, red spruce, specific heat, 113

Wood, spruce, balsam, heat of combustion, 113

Wood, spruce, Norway, heat of combustion, 113

Wood, spruce pine, specific heat, 113

Wood, spruce, red, specific heat, 113

Wood, sugar maple, specific heat, 110

Wood, western hemlock, specific heat, 109

Wood, white elm, specific heat, 108

Wood, white pine, heat of combustion, 112

Wool, heat of combustion, 114

Wool acetate, heat of combustion, 114

Wool fat, heat of combustion, 36

Wool, B-naphthalenesulfonate, heat of combustion, 114

Wool oxalate, heat of combustion, 114

Wool picrate, heat of combustion, 115

Wool protein, heat of combustion, 115

Wool sulfate, heat of combustion, 115

Wool p-toluenesulfonate, heat of combustion, 115

X14, propellant, specific heat, 94

$x y l a n$, heat of combustion, 93

Yolk, egg, heat of combustion, 88

Zeolite, silver $A$, specific heat, 115

Zeolite, sodium $A$, specific heat, 115

Zircaloy-2, heat of transition, 2

Zircaloy-2, specific heat, 2 


\section{REFERENCES}

(1) Bunting, J.G., Ashworth, T., and Steeple, H., Cryogenics

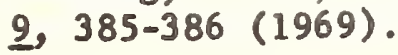

(2) 1968 Book of ASTM Standards with Related Materia 1, Part 17, Petroleum Products, Standard Method for Estimation of Net Heat of Combustion of Aviation Fuels ASTM Designation D1405-64. (published by ASTM, 1916 Race Street, Philadelnhia, PA. 19103).

(3) Mazur, J., and Zacharko, W., Acta Phys. Polon, 32, 501-514 (1967).

(4) Jessup, R. S., and Prosen, E. J., J. Research NBS 44, 387-393 (1950).

(5) Decker, L. J., Ward, J. R., and Freedman, E., Thermoch1m. Acta 8, $177-183$ (1974).

(6) Good, W. D., and Scott, D. 'ं., Chapter 2, "Combustion in a Bomb of Organic Fluorine Compounds", In Experimental Thermochemistry, Vol. 2, H. A. Skinner, Ed., (Interscience Publishers, New York, London 1962).

(7) Gardos, M., Rev. Scl. Instrum. 43, 1679-1687 (1972).

(8) Robie, R. A., Hemingway, B. S., and Wilson, W. H., Science 167 749-750 (1970); see also Proceedings of the Apollo 11 Lunar Science Conference, Geochim. Cosmoschim. Acta, Supp1. 1, 3, 2361-2367.

(9) Mer111, A. L., and Watt8, B. K., Agrlculture Handbook No. 74 (U. S. Government Printing Office, Washington, D. C., 1955) 105 pp; taken from: Atwater, W. O., and Bryant, A. P., The Avallability and Fuel Value of Food Materials, Conn. (Storrs) Agr. Expt. Station, 12th Ann. Rept. (1899), Pp. 73-110.

(10) Gattow, G., and Krebs, B., Z. Anorg. Allgem. Chem. 322, 113-128 (1963).

(11) "Water and Aqueous Solutions Structure, Thermodynamics, and Transport Processes.", R. A. Horne, Ed, (John Wiley and Sons, New York, 1972).

(12) Bromley, L. A., J. Chem. Eng. Data 13, 399-402 (1968).

(13) Martin, J. F., J. Iron Steel Inst. 204, 56 (1966). 
(14) Campbe11, A. N., and van der Kouwe, E. T., Can. J. Cliem. 46, $1287-1291$ (1968).

(15) Ward, J.R., AIAA J. 12, 107-108 (1974).

(16) Apollo 11, Pre11m. Science Rept., NASA SP-214, 132 (1969).

(17) Apollo 15, Prelim. Science Rept., NASA SP-289, 6-17 (1972).

(18) Haggerty, J. S., Cooper, A. R., and Heasley, J. H., Phys. Chem. Glasses $\underline{9}$, 47-51 (1968).

(19) Emery, A. G., and Bened1ct, F. G., Am. J. Physio1. 28, 301-307 (1911).

(20). Berthelot, M. P. E., and Viel1le, P., Ann. chim. et phys. [6] $10,455-463$ (1887).

(21) Karrer, P., and Fioroni, W., Helv. Chim. Acta 6, 396-401 (1923).

(22) Kozina, M. P., and Skuratov, S. M., Doklady Akad. Nauk SSSR 127, 561-563 (1959).

(23) Kachinskaya, 0. N., Vestn. Mosk. Univ., Ser. Mat. Mekh. Astron. Fiz. Khim. 12, 227-234 (1957).

(24) Good, W. D., Lacina, J. L., and McCullough, J. P., J. Am. Chem. Soc. 82, 5589-5591 (1960).

(25) Josh1, R. M., and Zwolinsk1, B. J., Macromolecule 1, 25-30 (1968).

(26) Jessup, R. S., and Cummings, A. D., J. Research NBS 13, 357-369 (1934).

(27) Magnus, A., 2. physik. Chem. [N.F.] 9, 141-161 (1956).

(28) Ivin, K. J., Keith, W. A., and Mackle, H., Trans. Faraday Soc. 55, 262-267 (1959).

(29) Parks, G. S., and Mosley, J. R., J. Chem. Phys. 17, 6y1-6y4 (194y).

(30) Parks, G. S., and Mosher, H. P., J. Polymer Sc1. (A) 1 , (6), 1979-1984 (1963). 
(31) Sinke, G. C., and Stul1, D. R., J. Phys. Chem. 62, 397-401 (1958).

(32) Good, W. D., Lacina, J. L., DePrater, B. L., and McCullough, J. P., J. Phys. Chem. 68, 579-586 (1964).

(33) Marx, P., Smith, C.W., Worthington, A. E., and Dole, M., J. Phys. Chem. 59, 1015-1019 (1955).

(34) Wilhoit, R. C., and Dole, M., J. Phys. Chem. 57, 14-21 (1953).

(35) Thais, R. R., and Kohn, J. P., J. Chem. Eng. Data 9, 546-547 (1964).

(36) Breitenbach, J. W., Terkosch, J., and Wessely, F., Monatsh. Chem. 83, 591-598 (1952).

(37) Hulden, B., Proc. Symp. Recovery Pulping Chem., 1st, $387-405$ (1968); (published 1969).

(38) Fisher, R. A., Brodale, G. E., Hornung, E. W., and Glauque, W. F., Rev. Sc1. Instr. 39, 108-114 (1968).

(39) Giauque, W. F., Fisher, R. A., Hormung, E. W., and Brodale, G. E., J. Chem. Phys. 58, 2621-2637 (1973).

(40) Splitstone, P. L., and Johnson, W. H., J. Research NBS 78A, $611-616(1974)$.

(41) Chang, S. S., and Bestu1, A. B., J. Research NBS 77A, 395-405 (1973).

(42) Chang, S.S., J. Research NBS 78A, 387-400 (1974).

(43) Gucker, F. T., Jr., and Ford, W. L., J. Am. Chem. Soc. 60, 2563 (1938).

(44) Perry, E. S., and Weber, W. H., J. Am. Chem. Soc. 71, 3726-3730 (1949).

(45) Perry, E. S., Weber, W. H., and Daubert, B. F., J. Am. Chem. Soc. 71, $3720-3726$ (1949).

(46) Clark, P. E., Waldeland, C. R., and Cross, R. P., Ind. Eng. Chem. $38,350-356(1946)$. 
(47) Poole, H., "The Calorific Power of Fuels", rewritten by R. T. Kent, 3rd Edit1on (John Wiley and Sons, Inc., New York, 1918).

(48) Lord, N. W., et a1., "Analysis of Coals in the United States, Part 1 - Analysis", U. S. Bureau of Mines Bullet in 22 (U. S. Government Printing Office, Washington, D. C., 1915).

(49) Halsam, R. T., and Russell, R. P., "Fuels and their Combustion", Chapter III. (McGraw-H111 Book Co., Inc., New York, 1926).

(50) White, A. H., and Barker, P., "Coals available for the Manufacture of Illuminating Gas", U. S. Bureau of Mines Bulletin 6, (U. S. Government Printing Office, Washington, D. C., 1911).

(51) Fernald, R. H., "Notes on the Uses of Low-Grade Fuel in Europe", U. S. Bureau of Mines Technical Paper 123 (U. S. Government Printing office, Washington, D. C., 1915).

(52) "Technical Data on Fuel", H. M. Splers, Ed., Fifth Edition (The Brit. Nat. Committee, World Power Conf., London, 1955).

(53) Davis, C. A., "The Uses of Peat", U. S. Bureau of Mines Bulletin 16 (U. S. Government Printing Office, Washington, D. C., 19 11).

(54) Wisely, F. E., Sutterfield, G. W., and Klumb, D. L., Proceedings of the Fourth Mineral Waste Utilization Symposium, E. Aleshin (co-sponsored by the U. S. Bureau of Mines and the IIT Research Institute) May 7, 8 (1974), Chicago, I11., Pp. 191-195.

(55) Yamada, T., N1ppon Noge1 Kagaku Ka1sh1, 44, 587-590 (1970).

(56) Mason, D. M., and Eakin, B. R., Inst. Gas Technol., Research Bul1. No. 3218 pp. (1961).

(57) Rastog1, R. P., Kishore, K., and Singh, G., AIA I J. 12, 9-10 (1974).

(58) Rastog1, R. P., Kishore, K., and Singh, G., Thermochim. Acta 12 , 89-96 (1975). 
(59) Herlet, A., and Reich, G., Z. Angew. Phys. 9, 14-23 (1957).

(60) Franz, J., Mische, H., and Kuzay, P., Plaste Kautsch. 14, $472-476(1967)$.

(61) Bliss, L. C., Ecology 43, 753-757 (1962).

(62) Kaiser, E. R., Proceedings of the 1966 National Incinerator Conference, ASME, 84-88 (1966).

(63) Duca, W. J., Trans Am. Foundrymen's Soc. 81, 108-109 (1973).

(64) Lewis, D. C., Frisch, M. A., and Margrave, J. L., Carbon 2, $431-432$ (1965).

(65) Zoller, P., Decker, P. R., and Dillinger, J. R., J. App 1. Phys. 40, 1964-1966 (1969).

(66) Loewen, E. G., Trans. ASME 78, 667-670 (1956).

(67) Deighton, M., J. Iron Stee 1 Inst. 208, 1012-1014 (1970).

(68) Matwe jew, G. M., Agarkow, A. S., and Kerbe, F., Silikattechnik $18,324-326$ (1967).

(69) Wun, M., and Phillips, N. E., Cryogenics $36-37$ (1975).

(70) Kreitman, M. M., Advan. Cryog. Eng. 16, $51-53$ (1970).

(71) Collins, L. W., Downs, W. R., Gibson, E. K., and Moore, G. W., Thermochim. Acta 10, 153-159 (1974).

(72) Setchkin, N. P., J. Research NBS 53, 49-66 (1954).

(73) Paine, R. T., Ann. Rev. Ecological Systems 2, 145-164 (1971).

(74) Mansson, M., Rapport, N., and Westrum, E. F., Jr., J. Am. Chem. Soc. 92, 7296-7299 (1970). 
(75) Slobodkin, L. B., and Richman, S., Nature 191, 299 (1961).

(76) Abramova, L. T., Abramov, V. G., and Merzhanov, A. G., Russ. J. Phys. Chem. 43, 647-650 (1969).

(77) Engineering Design Handbook, Explosive Series, Properties of Explosives of Military Interest, U. S. Army Material Command Pamphlet (AMCP) 706-177, January, 1971.

(78) Kanagy, J. R., J. Research NBS 55, 191-195 (1955).

(79) Marklund, R., Bystrom, S., Larsion, M., and Lindquist, T., Cryogenics 13, 671-672 (1973).

(80) Eldridge, E. A., and Deem, H. W., U. S. AEC BMI 1803, 17 PP. (1967).

(81) McMi111n, C. W., Wood Sc1. and Technol. 2, 233,-240 (1968).

(82) McMillin, C. W., Wood Sci. 2, 107-111 (1969).

(83) McMil1in, C.W., Wood Sc1. 3, 52-53 (1970).

(84) Kaiser, E. R., and Friedman, S. B., Combustion, 31-36, May, 1968.

(85) "Combustion Englneering", Fryling, G. R., Ed., rev, ed., Chapter 27, p. 3, (Combustion Eng. Inc., New York, 1966).

(86) Roberts, A. F., Combust. Flame 11, 437-441 (1967).

(87) Browne, F. I., U. S. Dept. Agri, Forest Service Res. Paper FPL-19, 16 pp. Dec. 1964.

(88) Parr, S. W., and Davidson, C. N., J. Ind. Eng. Chem. 14, 935-936 (1922).

(89) Gottlieb, E., J. prakt. Chem. 28, 385-421 (1883).

(90) Tavernier, $P_{\circ}$, and Lamouroux, M., Mem. poudres 39, 335-356 (1957).

(91) Ponomarev, V. V., Russ. J. Phys. Chem. 40, 600-601 (1966). 
(92) Suzuki, H., and Koyama, M., Cryogenics 15, 38-39 (1975).

(93) Koch, P., Wood Sc1. I, 203-214 (1969).

(94) "Asphalt Sclence and Technology", E. J. Barth (Gordon and Breash, Science Publishers, New York and London, 1962).

(95) Saal, R., Heukelom, W., and Blokker, P., J. Inst. Petroleum 26, (No. 195), 29-39 (1940).

(96) Vucelic, D., Vucelic, V., and Juranic, N., J. Themal Anal. 5, 459-463 (1972).

(97) Douglas, T. B., and Dever, J. L., J. Research NBS 54, 15-19 (1955).

(98) Lenoir, J. M., and Hipkin, H. G. J. Chem. Eng. Data 18, $195-202(1973)$.

(99) Scanes, F.S., and Martin, R. A. M., Combust. Flame 23; 357-362 (1974).

(100) Bowring, R.W., Garton, D. A., and Norris, H. F., Atomic Energy Establishent, Winfrith, Dorchester (Great Britain) AEEW-R-38, 23 PP. (1960).

(101) Rydholm, S. A., Pulp and Paper Mag. Canada, T2-T15. Jan, 1967.

(102) Gullichsen, J., Proc. Sym. Recovery of Pulping Chemicals, Helsink1 221-225 (1968).

(103) Martin, W. H., and Rixon, J., J.Sc1. Instruments 36, 179-182 (1959).

(104) Bromley, I. A., Diamond, A. E., Salami, E., and Wilkins, D. G., J. Chem. Eng. Data 15, 246-253 (1970).

(105) Domalski, E. S., and Armstrong, G. T., J. Research NBS 71A, 105-117 (1967). 
(106) Furukawa, G. T., McCoskey, R. E., and King., G. J., J. Research NBS 49, 273-278 (1952).

(107) Suurkuusk, J., and Wadso, I., J. Chem. Thermodynamics 6 , 667-679 (1974).

(108) Furukawa, G. T., and McCoskey, R. E., J. Research NBS 51, 321-326 (1953).

(109) Persson, A., Ark. Fys. 23, 229-234 (1963).

(110) Westrum, E. F., Jr., Chien Chow, Osborne, D. W., and Flotow, H. E., Cryogenics I, 43-44 (1967).

(111) Karrer, P., and Fioroni, W., Ber. deut. chem. Ges. 55B, 2854-2863 (1922).

(112) Roth, W. A., and Becker, F., "Kalorimetrisohe Methoden zur Bestimmung Chemischer Reaktionswarmen" P. 75 (F. Vleweg \& Sohn, Braunschwelg, 1956).

(113) Boyd, C. E., Ecology 51, 902-906 (1970).

(114) Aleksandrov, V. V., Buletov, N. S., Pastukhova, T. V., and Tukhaev, R. K., Fiz. Goreniya Vzryva 9, 75-83 (1973).

(115) Roth, W. A., and Wallasch, H., Ann. 407, 134-145 (1915).

(116) Berlin, E., Kliman, P. G., and Pallansch, M. J., Thermochim. Acta 4, 11-16 (1972).

(117) Pässler, W., and König, W., Z. angew. Chem. 44, 288-291, 304-309 (1931).

(118) Jaeger, F. M., and Rosenbohm, E., Rec. trav. chim. 47, 513-557 (1928).

(119) Romanova, M. V., Smi nnov, I. A., and Tikhonov, V. V., Fiz. Tverd. Tela. 13, 1812-1814 (1971).

(120) Chang, S. S., and Bestu1, A. B., J. Research NBS 77A, 395-405 (1973).

(121) Furukawa, G. T., McCoskey, R. E., and Reilly, M. L., J. Research NBS, 55, 127-132 (1955). 
(1:2) Furukawa, G. T., McCoskey, R. E., and King, G. J., J. Research NBS 50, 357-365 (1953).

(123) Stroud, J.W., Ph.D. Thesis, Cornell University, (1961).

(124) Paladines, 0. L., Reid, J. T., Bensadoun, A., and Van Niekerk, B. D. H., J. Nutrition 82, 145-149 (1964).

(125) Henning, 0., and Strobel, U., Wiss. Z. Hochsch. Architekt. Bauw. Weimar. 17, 354-355 (1970).

(126) Chang, Y. A., and Huitgren, R., J. Chem. Eng. Data 12, 98-99 (1967).

(127) Bair, H. E., Soc. Plast. Eng. Annu. Tech. Conf. Tech. Pap. 28th 16, 115-117 (1970).

(128) Hutchens, J. O., Cole, A. G., and Stout, J.W., J. Biol. Chem. 244, 26-32 (1969).

(129) Downer, L., and Gardiner, K. E. S., J. Inst. Petroleum 58, $6-13$ (1972).

(130) Cragoe, C. S., Bur. Standards M1sc. Publ. No. y7, (1929).

(131) Stull, D. R., Westrum, E. F。, Jr., and Sinke, G., "Thermodynamics of Organic Compounds" (J. Wiley and Sons, Inc., New York, 1969).

(132) Cox, J. D., and P1lcher, G., "Thermochemistry of Organic and Organometallic Compounds" (Academic Press, Inc., Publishers, New York and London, 1970).

(133) Ornellas, D. I., J. Phys. Chem. 72, 2390-2394 (1968).

(134) Wegman, D. D., Evans, W. H., Parker, V. B., Halow, I., Bailey, S. M., and Schumm, R. H., NBS Technical Note 270-3, (1968).

(135) Henkin, H., and McGil1, R., Ind. Eng. Chem. 44, 1391-1395 (1952).

(136) Wagman, D. D., Evans, W. H., Parker, V. B., and Schumm, R. H., NBSIR 76-1034 (1976). 
(137) Meier, A., and Meyerhof, 0., Blochem. Z. 150, 233-252 (1924).

(138) Krien, G., Licht, H. H., and Zlerath, J., Thermochim. Acta $\underline{6}$, 465-472 (1973).

(139) Dunlap, F., Forest Service, U. S. Department of Agriculture, Bull. 110, 28p. (1912).

(140) Backhurst, J. R., and Harker, J. H., Fuel 52, 302-303 (1973).

(141) ASME Research Comittee on Industr1al and Municipal Wastes, "Combustion Fundamentals for Waste Incineration," 212p. (American Soclety of Mechanical Englneers, New York, 1974).

(142) Dewey, P.H., Harper, D. R., J. Research NBS 21, 457-474 (1938). 


\section{SYMBOLS FOR THERMOOYNAMIC QUANTITIES}

The symbols indicated in this Appendix are those adopted by the International Union of Pure and Applied Chemistry (IUPAC), 1969.

Symbols in parentheses are alternates, accepted but not recommended by IUPAC. Also indicated are other commoniy used symbols and names of quantities not accepted by IUPAC.

Primary Symbols

$T$ thermodynamic temperature (absolute temperature)

$t, \theta \quad$ Celsius temperature

R molar gas constant

k Boltzmann constant

$q, Q$ heat

$W, W$ work

$U,(E)$ internal energy

$H$ enthalpy: $H=U+p V$

$S$ entropy

A Helmholtz energy: $A=U$ - TS

$\mathrm{J}$ Massieu function: $J=-A / T$

$G \quad$ Gibbs energy: $G=H-T S$

NOTE: The Gibbs energy is frequently called free energy, Gibbs free energy, or Gibbs function. The commonly encountered term free energy in American work on thermodynamics almost invariably refers to Gibbs energy. This usage of the term free energy and the symbol $F$ are being discouraged by the IUPAC because of confusion with the Helmholtz energy and the common European usage of $F$ for the latter.

$Y \quad$ Planck function: $Y=-G / T$

$Z$ compressibility factor: $Z=p V / n R T$

C heat capacity

c specific heat capacity (heat capacity divided by mass)

$\gamma,(k)$ heat capacity ratio: Cp/ch

$\mu \quad$ Joule-Thomson coefficient

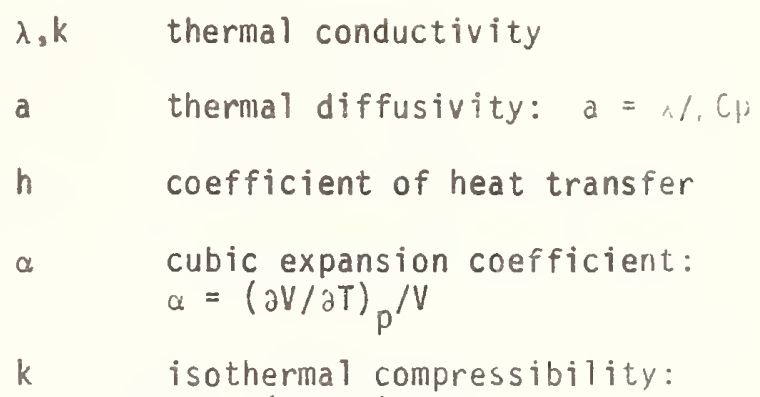

Selected Symbols for Other Quantities in Thermodynamics

$\begin{array}{ll}A, S & \text { area } \\ y & \text { volume } \\ t & \text { time } \\ m & \text { mass }\end{array}$
"molecular weight") 


\begin{tabular}{|c|c|}
\hline$L, N_{A}$ & Avogadro constant \\
\hline N & number of molecules \\
\hline$n,(v)$ & amount of substance \\
\hline$x_{B}, y_{B}$ & $\begin{array}{l}\text { mole fraction of substance } B: \\
x_{B}=n_{B} / \Sigma_{j} r_{i}\end{array}$ \\
\hline$w_{B}$ & mass fraction of substance $B$ \\
\hline$\phi_{B}$ & volume fraction of substance $B$ \\
\hline$m_{B}$ & $\begin{array}{l}\text { molality of solute substance } B \\
\text { (amount of } B \text { divided by the mass of } \\
\text { solvent) }\end{array}$ \\
\hline$C_{B},[B]$ & $\begin{array}{l}\text { concentration of substance B (amount } \\
\text { of B divided by the volume of the } \\
\text { solution) }\end{array}$ \\
\hline$\rho_{B}$ & $\begin{array}{l}\text { mass concentration of substance } B \\
\text { (mass of } B \text { divided by the volume of } \\
\text { the solution) }\end{array}$ \\
\hline$v_{B}$ & $\begin{array}{l}\text { stoichiometric coefficient of sub- } \\
\text { stance } B \text { (negative for reactants, } \\
\text { positive for products). (The general } \\
\text { equation for a chemical reaction is - } \\
\Sigma_{\mathrm{B}}{ }_{\mathrm{B}} \mathrm{B}=0, \text { [Example: for the reaction } \\
\mathrm{H}_{2} \mathrm{O} \rightarrow \mathrm{H}_{2}+\frac{1}{2} \mathrm{O}_{2}, \mathrm{H}_{2}+\frac{1}{2} \mathrm{O}_{2}-\mathrm{H}_{2} \mathrm{O}=0 \text { ] }\end{array}$ \\
\hline$\zeta$ & extent of reaction: $\left(d_{\zeta}=d n_{b} / \nu_{B}\right)$ \\
\hline k & equilibrium constant \\
\hline$a$ & degree of dissociation \\
\hline$z_{B}$ & $\begin{array}{l}\text { charge number of an ion B (positive } \\
\text { for cations, negative for anions) }\end{array}$ \\
\hline
\end{tabular}


UNITS OF MEASUREMENT - INTERNATIONAL SYSTEM (SI)

\section{SI - BASE UNITS AND SUPPLEMENTARY UNITS}

\begin{tabular}{lll}
\multicolumn{1}{c}{ quantity } & name of unit & symbol \\
\hline length & metre & $\mathrm{m}$ \\
mass & kilogram & $\mathrm{kg}$ \\
time & second & $\mathrm{s}$ \\
temperature & kelvin & $\mathrm{K}$ \\
electric current & ampere & $\mathrm{A}$ \\
luminous intensity & candela & $\mathrm{cd}$ \\
amount of substance & mole & mol \\
plane angle & radian & rad \\
solid angle & steradian & $\mathrm{sr}$ \\
\hline
\end{tabular}

\section{DEFINITIONS OF THE SI BASE UNITS}

metre: The metre is the length equal to 1650763.73 wavelengths in vacuum of the radiation corresponding to the transition between the levels $2 p_{10}$ and $5 d_{5}$ of the krypton-86 atom.

kilogram: The kilogram is equal to the mass of the international prototype of the kilogram.

second: The second is the duration of 9192631770 periods of the radiation corresponding to the transition between the two hyperfine levels of the ground state of the cesium-133 atom.

kelvin: The kelvin is the fraction 1/273.16 of the thermodynamic temperature of the triple point of water. The kelvin is used both for thermodynamic temperature and for thermodynamic temperature interval.

ampere: The ampere is that constant current which, if maintained in two straight parallel conductors of infinite length, of negligible cross section, and placed 1 metre apart in vacuum, would produce between these conductors a force equal to $2 \times 10^{-7}$ newton per metre of length.

candela: The candela is the luminous intensity, in the perpendicular direction, of a surface of $1 / 600000$ square metre of a black body at the temperature of freezing platinum under a pressure of 101325 newtons per square metre.

mole: The mole is the amount of substance of a system which contaills as many elementary entities as there are carbon atoms in $0.01 \%$ kilogram of carbon-12.

NOTE: The elementary entities must be specified and may be atoms, molecules, ions, electrons, other particles, or specified groups of such particles.

\section{PREFIXES}

SI Symbol Prefix name Multiplication factor

$\begin{array}{lll}T & \text { tera } & 10^{12} \\ G & \text { giga } & 10^{9} \\ M & \text { mega } & 10^{6} \\ k & \text { kilo } & 10^{3} \\ h & \text { hecto } & 10^{+} \\ \text {da } & \text { deka } & 10^{+1} \\ d & \text { deci } & 10^{-1} \\ c & \text { centi } & 10^{-2} \\ m & \text { milli } & 10^{-3} \\ \mu & \text { micro } & 10^{-6} \\ n & \text { nano } & 10^{-9} \\ \mathrm{p} & \text { pico } & 10^{-12} \\ f & \text { femto } & 10^{-15} \\ \text { a } & \text { atto } & 10^{-18}\end{array}$


OTHER UNITS EXACTLY DEFINED IN TERHS OF SI UNITS (PARTIAL LISTING)

\begin{tabular}{|c|c|c|c|}
\hline Quantity & Name & Symbol & Definition \\
\hline length & inch & in. & $2.54 \times 10^{-2} \mathrm{~m}$ \\
\hline mass & pound (av.) & $1 b$ & $0.45359237 \mathrm{~kg}$ \\
\hline force & kilogram force & $\mathrm{kgf}$ & $9.80665 \mathrm{~N}$ \\
\hline pressure & atmosphere & $\mathrm{atm}$ & $101325 \mathrm{~N} \cdot \mathrm{m}^{-2}$ \\
\hline pressure & torr & Torr & $(101325 / 760) \mathrm{N} \cdot \mathrm{m}^{-2}$ \\
\hline pressure & $\begin{array}{l}\text { millimetre of } \\
\text { mercury }\end{array}$ & $\mathrm{mmHg}$ & $\begin{array}{l}13.595] \times 980.665 \\
\times 10^{-2} \mathrm{~N} \cdot \mathrm{m}^{-2}\end{array}$ \\
\hline energy & kilowatt-hour & kWh & $3.6 \times 10^{6} \mathrm{~J}$ \\
\hline energy & $\begin{array}{l}\text { thermochemical } \\
\text { calorie*** }\end{array}$ & $\mathrm{cal}_{\text {th }}$ & $4.184 \mathrm{~J}$ \\
\hline energy & IT calorie** & $\mathrm{cal}_{\text {IT }}$ & $4.1868 \mathrm{~J}$ \\
\hline energy & $\begin{array}{l}\text { IT British thermal } \\
\text { unit** }\end{array}$ & Btu $_{\text {IT }}$ & $1055.06 \mathrm{~J} \star$ \\
\hline $\begin{array}{l}\text { temperature } \\
\text { (thermodynamic) }\end{array}$ & degree Rankine & ${ }^{\circ} \mathrm{R}$ & $j / 9 \mathrm{~K} * \star \star \star *$ \\
\hline temperature & degree Celsius & ${ }^{\circ} \mathrm{C}$ & 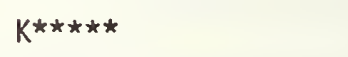 \\
\hline temperature & degree Fahrenheit & ${ }^{\circ} \mathrm{F}$ & $5 / 9 K^{* \star \star \star \star \star}$ \\
\hline volume & litre & 1 & $10^{-3} \mathrm{~m}^{3}$ \\
\hline
\end{tabular}

* The exact relationship is: 1 Btu $_{\text {IT }}=(4.1868 / 1.8) \times 453.59237$

** IT refers to International Tables (International Steam Tables).

*** The thermochemical calorie is used in tables in this monograph.

**** $\left[T\left({ }^{\circ} R\right)=1.8 T(K)\right] ;\left[T\left({ }^{\circ} R\right)=t\left({ }^{\circ} \mathrm{F}\right)+459.67\right] ;\left[T\left({ }^{\circ} R\right)=1.8 t\left({ }^{\circ} \mathrm{C}\right)+491.67\right]$.

$* \star \star \star \star *\left[t\left({ }^{\circ} \mathrm{C}\right)=T(K)-273.15\right]$.

$* * \star \star \star *\left[t\left({ }^{\circ} \mathrm{F}\right)=1.8 \mathrm{t}\left({ }^{\circ} \mathrm{C}\right)+32\right]$.

\section{Auxiliary Conversion Factors}

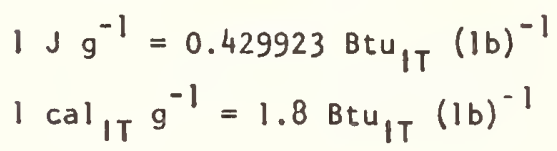


The physical constants indicated below are excerpted from a list recommended by the National Bureau of Standards. The complete list is found in National Bureau of Standards Technical News Bulletin, October, 1963.

\pm Error limits are approximately $3 \mathrm{stan}$ dard deviations and apply to last digits of the value.

PHYSICAL CONSTANTS

\section{Constant}

Speed of light in vacuum

Elementary charge

Avogadro constant

Electron rest mass

Proton rest mass

Neutron rest mass

Faraday constant

Planck constant

Rydberg constant

Bohr radius

Electron radius

Gas constant

Normal volume perfect gas

Boltzmann constant

First radiation constant

Second radiation constant

Wien displacement constant

Stefan-Boltzmann constant

Energy associated with -

Electron volt

Frequency (electromagnetic)

Wavelength (electromagnetic)

Wave number

Kelvin
eV $\quad 1.60210$

$\mathrm{e} / \mathrm{h}$

2.41804

$\mathrm{ch} / \mathrm{e}$

1.23981

$\mathrm{e} / \mathrm{ch}$

8.06573

Symbol Value

2.997925

1.60210

6.02252

9.1091

1.67252

1.67482

9.64870

6.6256

1.0973731

5.29167

2.81777

8.3143

2.24136

1.38054

3.7415

1.43879

2.8978

5.6697

e/k

1.16049
A more recent iist of constants aitfering by small amounts from the above values, is under consideration by the Task Group on Fundamental Constants of the Committee on Data for Science and Technology (International Council on Scientific Unions). The new values are not significantly different from those with which this handbook was prepared. (See NBS Special Publications 344, March, 1971.)
Error Limit

$$
3
$$$$
7
$$$$
28
$$$$
4
$$$$
8
$$$$
8
$$$$
16
$$$$
5
$$$$
3
$$$$
7
$$

30

18

3

4

29

\section{Units (SI)}

$$
m \cdot s^{-1}
$$

$10^{19} \mathrm{C}$

$10^{23} \mathrm{~mol}^{-1}$

$10^{-31} \mathrm{~kg}$

$10^{-27} \mathrm{~kg}$

$10^{-27} \mathrm{~kg}$

$10^{4_{4}} \mathrm{~mol}^{-1}$

$10^{-34} \mathrm{~J} \cdot \mathrm{s}$

$10^{-11} \mathrm{~m}^{-1}$

$10^{-11} \mathrm{~m}$

$10^{-15} \mathrm{~m}$

$10^{0} \mathrm{~J} \cdot \mathrm{K}^{-1} \cdot \mathrm{mol} \mathrm{I}^{-1}$

$\left.10^{-2} \mathrm{~m}^{3} \cdot \mathrm{mol}\right]^{-1}$

$10^{-23} \mathrm{~J} \cdot \mathrm{K}^{-1}$

$10^{-16} \mathrm{~W} \cdot \mathrm{m}^{2}$

$10^{-2} \mathrm{~m} \cdot \mathrm{k}$

$10^{-3} \mathrm{~m} \cdot \mathrm{K}$

$10^{-8} \quad k \cdot m^{-2} \quad k^{-14}$

7

7

4

23

16
$10^{-19} \mathrm{~J} \cdot(\mathrm{eV})^{-1}$

$10^{14} \mathrm{~Hz} \cdot(\mathrm{eV})^{-1}$

$10^{-6} \mathrm{eV} \cdot \mathrm{m}$

$10^{5} \mathrm{~m}^{-1} \cdot(\mathrm{eV})^{-1}$

$10^{4} \quad K \cdot(e V)^{-1}$ 
SI DERIVED UNITS (PARTIAL LISTING)

\begin{tabular}{|c|c|c|c|c|}
\hline & & $\begin{array}{l}\text { Symbol } \\
\text { for SI unit }\end{array}$ & $\begin{array}{l}\text { Definition } \\
\text { of SI unit }\end{array}$ & $\begin{array}{c}\text { Other } \\
\text { Definitions } \\
\end{array}$ \\
\hline force & newton & N & $\mathrm{m} \cdot \mathrm{kg} \cdot \mathrm{s}^{-2}$ & \\
\hline pressure & pascal & $\mathrm{Pa}$ & $\mathrm{m}^{-1} \cdot \mathrm{kg} \cdot \mathrm{s}^{-2}$ & $N \cdot m^{-2}$ \\
\hline energy & joule & $\mathrm{J}$ & $\mathrm{m}^{2} \cdot \mathrm{kg} \cdot \mathrm{s}^{-2}$ & $N \cdot m$ \\
\hline power & watt & W & $\mathrm{m}^{2} \cdot \mathrm{kg} \cdot \mathrm{s}^{-3}$ & $J \cdot \mathrm{s}^{-1}$ \\
\hline electric charge & coulomb & c & $A \cdot S$ & \\
\hline electric potential difference & volt & v & $\mathrm{m}^{2} \cdot \mathrm{kg} \cdot \mathrm{s}^{-3} \cdot \mathrm{A}^{-1}$ & $W \cdot A^{-1}$ \\
\hline electric resistance & ohm & $\Omega$ & $\mathrm{m}^{2} \cdot \mathrm{kg} \cdot \mathrm{s}^{-3} \cdot \mathrm{A}^{-2}$ & $V \cdot A^{-1}$ \\
\hline electric conductance & siemens & $\mathrm{S}$ & $m^{-2} \cdot \mathrm{kg}^{-1} \cdot \mathrm{s}^{3} \cdot \mathrm{A}^{2}$ & $A \cdot V^{-1}$ \\
\hline electric capacitance & farad & $F$ & $\mathrm{~m}^{-2} \cdot \mathrm{kg}^{-1} \cdot \mathrm{s}^{4} \cdot \mathrm{A}^{2}$ & $C \cdot v^{-1}$ \\
\hline magnetic flux & weber & $w b$ & $\mathrm{~m}^{2} \cdot \mathrm{kg} \cdot \mathrm{s}^{-2} \cdot \mathrm{A}^{-1}$ & $v \cdot s$ \\
\hline inductance & henry & $H$ & $\mathrm{~m}^{2} \cdot \mathrm{kg} \cdot \mathrm{s}^{-2} \cdot \mathrm{A}^{-2}$ & $W b \cdot A^{-1}$ \\
\hline magnetic flux density & tesla & $T$ & $\mathrm{~kg} \cdot \mathrm{s}^{-2} \cdot \mathrm{A}^{-1}$ & $w b \cdot m^{-2}$ \\
\hline luminous flux & lumen & $1 \mathrm{~m}$ & $c d \cdot s r$ & \\
\hline illumination & $\operatorname{lux}$ & $1 x$ & $m^{-2} \cdot c d \cdot s r$ & \\
\hline trequency & hertz & $\mathrm{Hz}$ & $s^{-1}$ & \\
\hline
\end{tabular}


The atomic weights indicated below are those used in calculation of formula weights of the substances 7 isted in the table. Since the data were calculated more recent tables of atomic weights (Pure and Applied Chemistry $21,91-108$ (1970); 30, 639-649 (1972): 37 , 59]-603 (1974); 47, 75-95 (1976) have been recommended by the Commission on Atomic Weights of the International Union of Pure and Applied Chemistry (IUPAC). The changes which occur as a result of the new atomic weights ar not significant for the purposes for which this handbook was prepared, and so no changes have been made in the tables. This keeps the values consistent with those in "Combustion Fundamentals for Waste Incineration" [141].

Elements and their new atomic weights, which occur most prominently in the tables of thermodynamic data contained in this report, are: carbon, 12.011; and hydrogen, 1.0080. of lesser importance are: sulfur, 32.06 ; bromine, 79.904; iodine, 126.9045, and boron, 10.81. Minor changes have also been made in the atomic weights of certain metals.

\section{ATOMIC WEIGHTS (RELATIVE ATOMIC MASSES) OF THE ELEMENTS (1961)}

Name

Actinium

Aluminum

Americium

Antimony

Argon

Arsenic

Astatine

Barium

Berkelium

Beryllium

Bismuth

Boron

Bromine

Cadmium

Calcium

Cal ifornium

Carbon

Cerium

Cesium

Chlorine

Chromium

Cobalt

Copper

Curium

Dysprosium

Einsteinium

Erbium

Europium

Fermi um
Atomic

Symbol

number

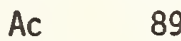

A1 13

Am $\quad 95$

Sb 51

Ar 18

As 33

At 85

$\mathrm{Ba} \quad 56$

Bk 97

$\mathrm{Be}$

$\mathrm{Bi}$

B

$\mathrm{Br}$

$\mathrm{Cd}$

Ca

$\mathrm{Cf}$

c

$\mathrm{Ce}$

Cs

Cl

Co

$\mathrm{Cu}$

$\mathrm{Cm}$

Dy

Es

Er

Eu
Name

Fluorine

Francium

Gadol inium

Gallium

Germani um

Gold

Hafnium

Helium

Holmium

Hydrogen

Indium

Iodine

Iridium

Iron

Krypton

Lanthanum

Lead

Lithium

Lutetium

Magnesium

Manganese

Mendelevium

Mercury

Molybdenum

Neodymium

Neon

Neptuni um

Nicke 1

Niobium

Nitrogen

Nobelium

Osmium

Oxygen

Palladium

Phosphorus

Platinum

Plutonium

Polonium

Potassium

Praseodymium

Promethi um

Protactinium

Radium

Radon

Rhen ium

Rhodium

Rubidium

Ruthenium

Samarium

Scandium

Selenium

Silicon

Silver

Sodium

Strontium

Sulfur

Tantalum

Technetium
Atomic

Symbol number

Fr

Fr

$\mathrm{Ga}$

$\mathrm{Ge}$

Au

Hf

$\mathrm{He}$

Ho

$\mathrm{H}$

In

I

Ir

$\mathrm{Fe}$

$\mathrm{Kr}$

$\mathrm{La}$

$\mathrm{Pb}$

LU

$\mathrm{Mg}$

$\mathrm{Mn}$

Md

$\mathrm{Hg}$

Mo

Nd

$\mathrm{Ne}$

$\mathrm{Np}$

$\mathrm{Ni}$

$\mathrm{Nb}$

N

No

Os

0

$\mathrm{Pd}$

$P$

$P t$

Pu

Po

$\mathrm{K}$

$\mathrm{Pr}$

$\mathrm{Pm}$

$\mathrm{Pa}$

$\mathrm{Ra}$

$\mathrm{Rn}$

$\mathrm{Re}$

$\mathrm{Rh}$

$\mathrm{Rb}$

$\mathrm{Ru}$

Sm

$\mathrm{Sc}$

Se

Si

$\mathrm{Ag}$

162.50

167.26

151.96

........
Atomic Weight

18.9984

157.25

69.72

72.59

196.967

178.49

4.0026

164.930

1.00797

114.82

126.9044

192.2

55.847

83.80

138.91

207.19

6.939

174.97

24.312

54.9380

200.59

95.94

144.24

20.183

........

58.71

92.906

14.0067

…….

190.2

15.9994

106.4

30.9738

195.09

........

39.102

140.907

.......

$\ldots \ldots \ldots$

$\ldots \ldots \ldots$

$\ldots \ldots \ldots$

186.2

102.905

85.47

101.07

150.35

44.956

78.96

28.086

107.870

22.9898

87.62

32.064

180.948 


\begin{tabular}{|c|c|c|c|}
\hline Name & Symbol & $\begin{array}{l}\text { Atomic } \\
\text { number }\end{array}$ & Atomic Weight \\
\hline Tellurium & $\mathrm{Te}$ & 52 & 127.60 \\
\hline Terbium & Tb & 65 & 158.924 \\
\hline Thallium & TI & 81 & 204.37 \\
\hline Thorium & Th & 90 & 232.038 \\
\hline Thul ium & $T m$ & 69 & 168.934 \\
\hline Tin & Sn & 50 & 118.69 \\
\hline Titanium & $\mathrm{Ti}$ & 22 & 47.90 \\
\hline Tungsten & $W$ & 74 & 183.85 \\
\hline Uranium & U & 92 & 238.03 \\
\hline Vanadium & v & 23 & 50.942 \\
\hline Xenon & $x_{e}$ & 54 & 131.30 \\
\hline Ytterbium & $Y b$ & 70 & 173.04 \\
\hline Yttrium & $Y$ & 39 & 88.905 \\
\hline Zinc & $\mathrm{Zn}$ & 30 & 65.37 \\
\hline Zirconium & $\mathrm{Zr}$ & 40 & 91.22 \\
\hline
\end{tabular}


NBS.114A (REV. $(1.77)$

\begin{tabular}{|c|c|c|}
\hline $\begin{array}{l}\text { U.S. DEPT. OF COMM. } \\
\text { BIBLIOGRAPHIC DATA } \\
\text { SHEET }\end{array}$ & $\begin{array}{l}\text { 1. PUBLICATION OR REPORT NO. } \\
\text { NBSIR } 78=1479\end{array}$ & 3. Recipient's Accession No. \\
\hline \multicolumn{2}{|l|}{ 4. TITLE AND SUBTITLE } & $\begin{array}{l}\text { 5. Publication Date } \\
\text { August } 1978\end{array}$ \\
\hline \multicolumn{2}{|c|}{ Thermodynamic Data for Waste Incineration } & 6. Performing Organization Code \\
\hline \multicolumn{2}{|c|}{$\begin{array}{l}\text { 7. AUTHOR(S) } \\
\text { Eugene S. Domalski, William H. Evans and Thomas L. Jobe, Jr. }\end{array}$} & 8. Performing Organ. Report No. \\
\hline \multirow{2}{*}{\multicolumn{2}{|c|}{$\begin{array}{l}\text { 9. PERFORMING ORGANIZATION NAME AND ADDRESS } \\
\text { NATIONAL BUREAU OF STANDARDS } \\
\text { DEPARTMENT OF COMMERCE } \\
\text { WASHINCTON, D.C. } 20234\end{array}$}} & $\begin{array}{l}\text { 10. Project/Task/Work Unit No. } \\
5435151\end{array}$ \\
\hline & & $\begin{array}{l}\text { 11. Contsact/Grant No. } \\
\text { G-125-5 }\end{array}$ \\
\hline \multirow{2}{*}{\multicolumn{2}{|c|}{$\begin{array}{l}\text { 12. Sponsoring Organization Name and Complete Address (Stroot, City, Stato, ZIP) } \\
\text { American Society of Mechanical Engineers } \\
\text { Research Comittee on Industrial and Municipal Wastes } \\
\text { United Engineering Center } \\
345 \text { East } 47 \text { th Street - New York. N.Y. } 10017\end{array}$}} & $\begin{array}{l}\text { 13. Type of Report a Period } \\
\text { Corered } \\
1 \text { Jan. } 1975-31 \text { Dec.19 }\end{array}$ \\
\hline & & 14. Sponsoring Agency Code \\
\hline
\end{tabular}

16. ABSTRACT (A 200 word or less factual summary of most oi gnificant information. If document includer a alenificant bibliography or literature survey, mention th here.)

A table of thermodynamic data of 331 selected materials has been compiled for the purpose of providing engineers with information on materlals which are not easily identifiable by a stoichiometric formula. Examples of such materials are: foods, wood species, oils, animals, plants, polymers, etc. The table is arranged alphabetically according to the names of the various materials, and whenever possible, the chief components or all readily identiflable components are supplied. In addition, the physical state, the kind of thermodynamic property, specific-property values, and citations to a list of 142 references are furnished. In order to assist the reader with finding a specific material or property, a material name and property index has been put together. A section on Units and Definitions explains the various thermodynamic properties being reparted and the condition under which they apply. An appendix supplies auxiliary information on symbols for thermodynamic quantities, units, physical constants and atomic weights.

The table is oriented more toward engineers involved in the disposal of municipal wastes than any other group; however, applications in other engineering and scientific sectors can easily be made.

17. KEY VORDS (oix to twelve entries; alphabetical order; capitalize only the firat letter of the first key word unlese a proper name; soparated by semicolons)

Heat of combustion; heat of explosion; miscellaneous materials; specific heat; vapor pressure.

\begin{tabular}{|c|c|c|}
\hline $\begin{array}{l}\text { 18. AVAILABILITY Unlimited } \\
\square \text { For Official Distribution. Do Not Release to NTIS }\end{array}$ & $\begin{array}{l}\text { 19. SECURITY CLASS } \\
\text { (THIS REPURT) } \\
\text { UNCL ASCIFIED }\end{array}$ & $\begin{array}{l}\text { 21. NO. OF PAGES } \\
167\end{array}$ \\
\hline $\begin{array}{l}\square \text { Order From Sup. of Doc,, U.S. Governmeat Printing Office } \\
\text { Washingron, D.C. 20402, SD Stoct No. SN003-003 }\end{array}$ & 20. SECURITY CLASS & 22. Price \\
\hline $\begin{array}{l}\text { [X] Order From National Techaical Information Service (NTIS) } \\
\text { Springfield, Virginia 22151 }\end{array}$ & UNCLASSIFIED & $\$ 8.00$ \\
\hline
\end{tabular}



